\title{
Raman and conduction studies on carbon nanotube networks and cupric oxide nanostructures
}

by

Shrividya Ravi

A thesis

submitted to the Victoria University of Wellington

in fulfilment of the

requirements for the degree of

Doctor of Philosophy

in Physics.

Victoria University of Wellington 



\begin{abstract}
This thesis presents detailed temperature-dependent Raman and conduction studies on two materials: cupric oxide $(\mathrm{CuO})$ nanostructures and single-walled carbon nanotube (SWNT) networks. SWNT networks are a promising alternative to indium tin oxide as the transparent conducting material in electronic displays. A key factor that complicates fundamental studies on SWNT networks is surfactant residue. Our study utilises SWNTs (HiPCO) dispersed in volatile solvents, which can be removed by annealing to only $100{ }^{\circ} \mathrm{C}$. Using this unique solvent system, we have systematically studied charge transport mechanisms in surfactant-free networks using a percolation approach; where sample resistance can be controlled by the amount of deposited material. The chemical environment of these networks was found to be unchanged using Raman spectroscopy; i.e. film fabrication did not cause any significant doping of the network. Around 15 surfactant-free networks, with resistances between $300 \mathrm{k} \Omega$ and $8 \mathrm{k} \Omega$, were found to follow a 'universal' charge transport model. Most of the networks could be described by two dimensional variable range hopping (VRH) and thermal activation. The barrier energy or, $T_{0}$ parameter, for the VRH mechanism was independent of resistance with a value of $20 \times 10^{3}$ $\pm 9.4 \times 10^{3} \mathrm{~K}$. The activation energy also had a resistance-independent value of $160 \pm 20 \mathrm{meV}$. Four terminal measurements confirm that the activation mechanism is due to processes within the network not, Schottky barriers at the nanotube/metal interface. The effects of extrinsic adsorbants on the network resistance provides evidence for dominant non-metallic conduction pathways within the studied range of resistances. These results strongly suggest a characteristic barrier size in our SWNT networks where non-metallic tubes dominate the resistance.
\end{abstract}

The surfactant-free networks were also used to study the temperature-dependent behaviour of the radial breathing modes (RBM) in bundled nanotubes. Deconvolution of complex RBM spectra was made possible using an interactive routine: based on the higher resolution of second derivatives for fitting spectra with washed-out features. Using this routine, the temperaturedependent characteristics of the RBM lineshape could be identified. We find that RBM modes in our bundled networks soften at the same rate as individual tubes; the linewidth follows a three phonon decay process with a temperature-independent component and the intensity can be modelled from the change in $E_{i i}$ with temperature.

The second part of this thesis addresses the unique asymmetric lineshape of the $A_{1 g}$ mode in $\mathrm{CuO}$ nanowire forests. A symmetric lineshape is recovered at low powers indicating that the underlying mechanism is thermal. To study this effect, the high temperature behaviour of the $A_{1 g}$ mode is first analysed in a 'bulk' form of $\mathrm{CuO}$. The analytical temperature dependence of the $A_{1 g}$ mode frequency, linewidth and intensity were used as the basis of a physical model that 
connects lineshape asymmetry to laser-induced, spatial temperature gradients in the sample. The peak temperature (under the laser hotspot) was found to be proportional to laser power until it reaches a critical value. We believe that regions with temperature above the critical value cool by radiation rather than convection. 


\section{Acknowledgments}

First and foremost, I would like to thank my supervisors: Prof. Alan Kaiser and Dr. Chris Bumby for their valuable guidance and support. Chris, I am especially grateful for all the effort you have put into supervising me - with a full time job and two young kids! I am grateful to Prof. Pablo Etchegoin, Camille Artur and Matthias Meyer for helping me with the Raman spectrometer. My thesis wouldn't have nearly as much data in it if it weren't for Matthias Meyer, who not only introduced me to Python, but also generously assisted whenever I got stuck and needed help to get my head around the crazy serpentine language. A big thanks to David Flynn who took care of all the technicalities of the SEM. Another round of thanks goes to Dr. Ben Ruck and Jan Richter for helping me with the conductivity measurements on the Janis cryostat. I am particularly indebted to Dr. Simon Granville at IRL for his help and advice with the PPMS.

The $\mathrm{PhD}$ experience wouldn't be a happy one without some truly spectacular support. Of these people, Natalie Plank really helped through some tough periods. Natalie, you really did bring about a turning point (mental one!) in my $\mathrm{PhD}$. My parents, grandmother and sister took care of me on weekends and annoyed me enough (as only family can) that I often forgot my PhD troubles! And of course, Dmitri deserves a shower of Russian physics books/craft beer for eternity for his patience and kindness when I was at my most incorrigible.

I dedicate this thesis to Mushu: who will never read it and at best, experimentally chew on the pages for entertainment if ever this document comes his way. 


\section{Contents}

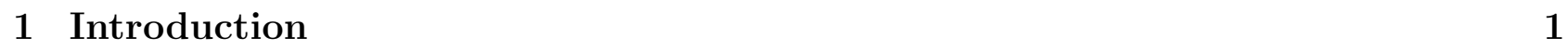

1.1 General overview . . . . . . . . . . . . . . . . . . . . . 1

1.2 Roadmap . . . . . . . . . . . . . . . . . . . . . . . 2

\begin{tabular}{|lll}
2 & Theory and experimental techniques & 5
\end{tabular}

2.1 Raman spectroscopy: theoretical aspects . . . . . . . . . . . . . . . . . 5

2.1 .1 Temperature dependence . . . . . . . . . . . . . . . 7

2.1 .2 Temperature profiles induced by gaussian beam . . . . . . . . . . . . . 13

2.2 Raman spectroscopy: experimental aspects . . . . . . . . . . . . . . . . 13

$2.2 .1 \quad$ LabRam optics . . . . . . . . . . . . . . . . . . . . . . . . . . . 14

2.2 .2 Gaussian laser beams . . . . . . . . . . . . . . . . . . . . . . . . . . . 15

2.2 .3 Laser power . . . . . . . . . . . . . . . . . . . . . 17

2.2 .4 Notch filter and spacer . . . . . . . . . . . . . . . . 17

2.2 .5 Objective . . . . . . . . . . . . . . . . . . . . 17

2.2 .6 Spectograph . . . . . . . . . . . . . . . . . . . . . 18

2.2 .7 Confocality $\ldots \ldots \ldots \ldots \ldots$

2.2 .8 Confocal pinhole effects . . . . . . . . . . . . . . . . . . 19

2.2 .9 Measuring temperature-dependent Raman spectra . . . . . . . . . . . . . 20

2.3 Conduction: theoretical aspects . . . . . . . . . . . . . . . . . . 20

2.3 .1 Percolation theory . . . . . . . . . . . . . . . . 20

2.3 .2 Conduction mechanisms $\ldots \ldots \ldots \ldots \ldots$. . . . . . . . . . . . 22

$2.3 .3 \quad$ Summary of conduction mechanisms in SWNT networks . . . . . . . . . 25

2.4 Conduction: experimental aspects . . . . . . . . . . . . . . . . . 27

2.4 .1 Contacts . . . . . . . . . . . . . . . . . . 27

2.4 .2 Measuring temperature dependent conduction . . . . . . . . . . . 28

2.5 X-ray diffraction $\ldots \ldots \ldots \ldots$

2.6 Other experimental techniques $\ldots \ldots \ldots \ldots \ldots$

$3 \quad$ Introduction to single-walled carbon nanotube networks 33

3.1 Introduction . . . . . . . . . . . . . . . . . . . . . . 33

3.2 Geometry and electronic structure . . . . . . . . . . . . . . . . . . 36

3.3 Tuning electronic properties of SWNT networks $\ldots \ldots \ldots$. . . . . . . . . . . 38 
3.4 Correlating percolation and charge transport models . . . . . . . . . . . . . . . 45

3.5 Sample diagnostics using Raman spectroscopy $\ldots$. . . . . . . . . . . . . . . . . . 46

4 Charge transport mechanisms in SWNT networks 49

4.1 Introduction . . . . . . . . . . . . . . . . . . . . . . . . . . . . . . . . . . . . 49

4.2 Film fabrication $\ldots \ldots \ldots \ldots$. . . . . . . . . . . . . . . . . . . . . . . . . . 49

$4.2 .1 \quad$ Nanotube dispersion $\ldots \ldots \ldots \ldots \ldots$. . . . . . . . . . . . . . . 49

4.2 .2 Film deposition . . . . . . . . . . . . . . . . . . . . . . . . 52

$4.3 \quad$ Spatial homogeneity of sprayed SWNT networks . . . . . . . . . . . . . . . 58

4.4 Conduction mechanisms . . . . . . . . . . . . . . . . . . . . . . . . 60

4.4 .1 Determining VRH dimensionality . . . . . . . . . . . . . . . . . 62

4.4 .2 Physical origin of activation conduction . . . . . . . . . . . . . . . 65

4.4 .3 Effect of clumps . . . . . . . . . . . . . . . . . . . 66

4.4 .4 Local irregularities in temperature-dependent resistance . . . . . . . . . . 67

4.4 .5 Summary statistics . . . . . . . . . . . . . . . 70

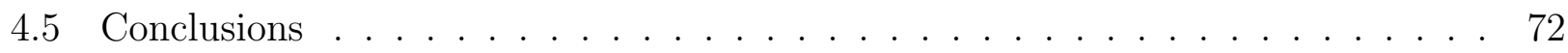

5 Raman spectroscopy of SWNT networks: literature review, methodology $\begin{array}{ll}\text { and characterisation } & 75\end{array}$

5.1 Introduction . . . . . . . . . . . . . . . . . . . . . . 75

5.2 The Raman spectrum of carbon nanotubes . . . . . . . . . . . . . . . . 77

5.2 .1 Kataura plot . . . . . . . . . . . . . . . . . 78

5.2 .2 Resonance effects . . . . . . . . . . . . . . . . . . . . . . . . . 79

5.3 Methodology: deconvoluting complicated Raman spectra . . . . . . . . . . . . . 82

5.3 .1 Introduction to second derivatives . . . . . . . . . . . . . . . 83

$5.3 .2 \quad$ Fitting spectra $\ldots \ldots \ldots \ldots \ldots$. . . . . . . . . . . . 84

$5.3 .3 \quad$ Fitting constraints $\ldots \ldots \ldots \ldots \ldots$. . . . . . . . . . . . 87

5.3 .4 Fitting accuracy . . . . . . . . . . . . . . . . 87

5.4 Methodology: statistical RBM analysis . . . . . . . . . . . . . . . . 88

5.4 .1 Calibration . . . . . . . . . . . . . . . . . . 90

5.4 .2 Analysing data from spatial maps . . . . . . . . . . . . . . . . . . . 92

5.5 Characterisation: effects of the film fabrication process . . . . . . . . . . . . . 93

5.6 Conclusions . . . . . . . . . . . . . . . . . . . . . . . . . . . . 95

\begin{tabular}{|lll}
6 & Temperature-dependent Raman spectroscopy of SWNT networks & 97
\end{tabular}

6.1 Introduction . . . . . . . . . . . . . . . . . . 97

6.2 Global heating . . . . . . . . . . . . . . . . . . . . 97

6.2 .1 Data collection . . . . . . . . . . . . . . . . . . . . . . 98

6.2 .2 Peak frequency $\ldots \ldots \ldots \ldots \ldots$. . . . . . . . . . . . . . . . . 98

6.2 .3 Peak linewidth . . . . . . . . . . . . . . . . . . . . . . . . . . . . . 102

6.2 .4 Peak intensity . . . . . . . . . . . . . . . . . . . 104

6.3 Local heating . . . . . . . . . . . . . . . . . . . . . . . 107 
6.3 .1 Data collection . . . . . . . . . . . . . . . . . . . . . . . 108

6.3 .2 Characteristics of local heating $\ldots \ldots \ldots \ldots . \ldots \ldots$

6.3 .3 Heating effects from spatial mapping . . . . . . . . . . . . . 110

6.4 Conclusions . . . . . . . . . . . . . . . . . . . . . . . . . . 111

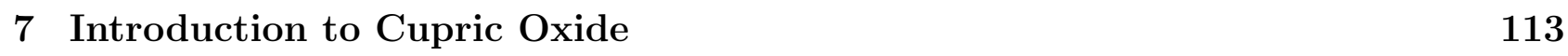

7.1 Introduction . . . . . . . . . . . . . . . . . . . . . . . . . . 113

7.2 Raman spectrum of $\mathrm{CuO} \ldots \ldots \ldots \ldots \ldots \ldots \ldots$

7.3 Physical origins of lineshape asymmetry $\ldots \ldots \ldots$

7.3 .1 Fano resonance . . . . . . . . . . . . . . . . . . . 120

7.3 .2 Phonon confinement . . . . . . . . . . . . . . . . . . . . . 121

7.4 Sample preparation and characterisation $\ldots \ldots \ldots \ldots \ldots$

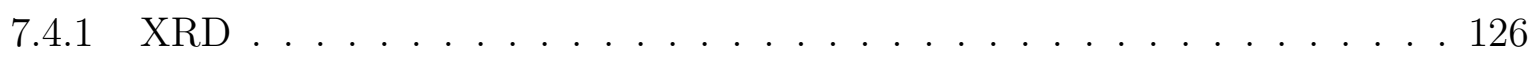

7.4 .2 Conduction in $\mathrm{CuO}$ thin films . . . . . . . . . . . . . . . . . 129

7.4 .3 Morphology . . . . . . . . . . . . . . . . . . . . . 130

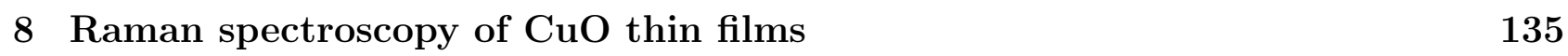

8.1 Introduction . . . . . . . . . . . . . . . . . . . . . . . . . 135

8.2 Temperature dependence of $\mathrm{CuO} \ldots \ldots \ldots \ldots$

$8.2 .1 \quad$ Peak position and linewidth . . . . . . . . . . . . . . . . 137

8.2 .2 Peak maximum and integrated intensities. . . . . . . . . . . . 140

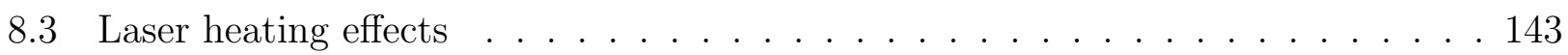

$8.3 .1 \quad$ Pellets . . . . . . . . . . . . . . . . . . . . . . . . . 143

8.3 .2 Thin films . . . . . . . . . . . . . . . . . . . . . . . . 144

8.3 .3 Extreme heating . . . . . . . . . . . . . . . . . . . 147

8.4 Conclusions . . . . . . . . . . . . . . . . . . . . . . . . . . 151

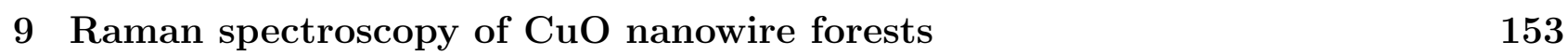

9.1 Introduction . . . . . . . . . . . . . . . . . . . . . . . . . 153

9.2 Data collection . . . . . . . . . . . . . . . . . . . . . . 154

9.3 Quantifying Asymmetry . . . . . . . . . . . . . . . . . . . . . 154

$9.3 .1 \quad$ Effective temperatures and asymmetry $\ldots \ldots \ldots \ldots \ldots$

9.4 Modelling lineshape asymmetry $\ldots \ldots \ldots \ldots \ldots$

$9.4 .1 \quad$ Model I: Inhomogeneous temperature distribution . . . . . . . . . . . 160

9.4.2 Model II: Inhomogeneous + Homogeneous temperature distribution . . . 167

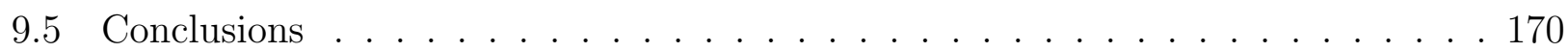

\begin{tabular}{ll}
\hline 10 Conclusions & 173
\end{tabular}

\begin{tabular}{ll}
\hline 11 Appendix 1 & 177
\end{tabular}

\begin{tabular}{lr}
\hline 12 Appendix 2 & 181
\end{tabular} 


\section{Chapter 1}

\section{Introduction}

\subsection{General overview}

Nanostructured forms of bulk materials have a number of advantages. The large surface area to volume ratio makes them ideal for applications which rely on surface properties: sensors, catalysts etc. The change in physical properties, particularly in the quantum confinement regime, creates new vistas for novel applications and research. For example, the indirect band-gap semiconductor, silicon, becomes a direct bandgap semiconductor when the quantised dimensions of the nanostructure become comparable to its Bohr radius. However, materials intended for mainstream technology face an additional criterion of cost. For most nanomaterials, the fiscal challenge lies in the fabrication. In this thesis, we study two nanomaterials which are considered cost-effective solutions for metallic and semiconductor device applications: single-walled carbon nanotube (SWNT) networks and cupric oxide $(\mathrm{CuO})$ nanowires respectively. Thus, the importance of nanomaterials in today's technological research landscape, has promoted nanomaterial characterisation as an important field of study. Among the many characterisation techniques available for probing deep into a material's properties, we have chosen two major techniques: Raman spectroscopy and temperature dependence of resistance.

Raman spectroscopy is a technique that probes a subset of a material's vibrational modes using monochromatic light. The power of this technique lies in its ability to impart information not only about the material's vibrational characteristics but also optical and electronic characteristics. As this technique relies on the optical absorption of light, analysis of the Raman signal can indicate changes in the material's optical characteristics. When the incoming photon energy is tuned to an electronic transition of the material, Raman can be used to infer certain properties of the material's electronic structure. Typically, changes to the vibrational, optical or electronic properties are studied in a comparative manner: as a function of some tunable parameter, e.g. temperature or pressure. In this thesis, we have focused primarily on the effects of temperature on the Raman signal from SWNT networks and cupric oxide nanowires.

The second major characterisation technique is temperature-dependent resistance measure- 
ments. This data is used to understand charge transport in a given material using various analytical models. For example, charge transport in metals can be described by the BlochGrüneisen model [1]. The parameters of this model are based on the dominant scattering mechanisms that affect the resistance, e.g. phonon-electron, residual defect or electron-electron scattering. In crystalline intrinsic semiconductors or extrinsic semiconductors with impurity levels, the behaviour of the temperature-dependent resistances typically follows thermal activation. The activation energy corresponds either to the bandgap or the energy difference between the impurity level and the dominant carrier band. In disordered or non-crystalline materials, models like fluctuation-induced tunnelling [2] and variable range hopping [3] are used to describe carrier transport over energy barriers between conducting segments within a material. In SWNT networks - a type of disordered, non-crystalline material - the size of these barriers and the mode by which carriers overcome them, indicate whether the conducting segments are metallic or non-metallic.

\subsection{Roadmap}

This thesis is comprised of ten chapters. Chapter 2 covers the theoretical and experiment aspects of Raman spectroscopy and temperature-dependent resistance studies. Additional characterisation techniques like X-ray diffraction and scanning electron microscopy are also covered in this chapter. The remainder of the thesis is split into two parts based on material type. Each part is self-contained, beginning with a detailed material-specific introduction followed by results.

The first part concerns SWNT networks. Chapter 3 provides the relevant theoretical details of SWNTs and SWNT networks. The chapter explains how the DC conductivity of SWNT networks can be tuned by chemical or physical means and the effects of these treatments on the charge transport mechanisms.

Chapter 4 covers our novel strategy for controlled, monitored fabrication of SWNT networks. The chapter describes the preparation and characterisation of 13 SWNT networks with resistances spanning almost two orders of magnitude: between 300 and $8 \mathrm{k} \Omega$. Conduction mechanisms for these samples are identified and discussed in detail.

Chapter 5 introduces Raman spectroscopy as a tool for studying SWNTs. The introduction discusses the gap in the literature for SWNT networks due to challenges in deconvoluting their complicated Raman spectra. The chapter then outlines two deconvolution techniques that address these challenges. Each technique serves a unique purpose. The first technique automatically fits a small spectral subset for a large volume of data e.g. spatial mapping studies. This technique is used as a diagnostic tool to characterise any chemical/physical consequences 
of our fabrication process on the SWNTs. The second technique allows for high resolution analysis of a single spectrum. The temperature dependence of the radial breathing modes in bundled SWNTs are studied using this technique in Chapter 6.

The following three chapters explore the temperature-dependent Raman signature of the $A_{1 g}$ mode in cupric oxide. Chapter 7 introduces cupric oxide as an interesting semiconducting material, particularly in its nanostructured forms. The chapter presents the primary reason for this set of studies: unusual asymmetry in the Raman lineshape of the $A_{1 g}$ mode from a $\mathrm{CuO}$ nanowire forest. The relevant theoretical details of asymmetric lineshapes are discussed here. The asymmetry seen in our samples is assigned to thermal phenomena.

The high temperature characteristics of the $A_{1 g}$ mode in 'bulk' $\mathrm{CuO}$ are presented in Chapter 8 . The chapter discusses the temperature dependence of the $A_{1 g}$ mode frequency, linewidth and intensity in analytical form. The effects of a global temperature are compared with local heating effects. The decomposition of $\mathrm{CuO}$ into $\mathrm{Cu}_{2} \mathrm{O}$ by intense local heating are also discussed.

Chapter 9 expands on the concept of Raman lineshape asymmetry caused by thermal phenomena; introduced in Chapter 7. A basic physical model, using the temperature dependencies established in Chapter 8, is derived to explain and fit the Raman lineshape. The chapter includes a discussion on the unexpected power dependence of the lineshape asymmetry in relation to cooling mechanisms. The thesis concludes with Chapter 10 which summarises the important findings. 


\section{Chapter 2}

\section{Theory and experimental techniques}

\subsection{Raman spectroscopy: theoretical aspects}

The Raman effect was first isolated and studied by the Indian physicist C.V. Raman [4, 5]. The benefit of this technique lies in the sheer wealth of molecular information that can be extracted by using monochromatic light. Today, the Raman effect is a widely-used tool used for classifying and analysing chemical compounds.

When photons from a monochromatic beam of light hit a sample, the majority undergo elastic scattering, also known as Rayleigh scattering. A small fraction of these photons experience inelastic collisions with phonons. Phonons are quanta of vibrations whose vibrational frequency is intricately dependent on the symmetry and constituent atoms of the molecule/crystal.

The Raman effect is the result of an inelastic interaction between a photon and an excitation e.g. a phonon. When the photon loses energy via an inelastic collision to a phonon mode, the resultant photon leaves with an energy shifted by $E_{\text {phonon }}\left(\hbar \omega_{\text {phonon }}\right)$. This precise change in energy results in sharp spectral features compared to spectroscopic techniques like UV-Vis where photon interactions are generally broad and featureless. The Raman shift is usually measured in wavenumbers $\left(\mathrm{cm}^{-1}\right)$ and is taken as the difference between the incident and scattered photon frequencies $\left(1 / \lambda_{\text {inc }}-1 / \lambda_{\text {scatt }}\right)$ where $\lambda$ is the photon wavelength in $\mathrm{cm}$. The position and intensity of the peaks displayed on the spectrum can be used as a chemical signature.

The selection criterion for Raman scattering is a non-zero change in polarisability with vibrational motion. The polarisation $(\mathbf{P})$ of a molecule as a function of electric field $(\mathbf{E})$ is given as: $\mathbf{P}=\alpha \mathbf{E}$ where $\alpha$ is the polarisability. Certain molecular vibrations such as the one shown in Figure 2.1 change the polarisability so that $\mathbf{P}$ is now given as:

$$
\mathbf{P}=\alpha \mathbf{E}+\frac{\delta \alpha}{\delta Q} Q \quad \mathbf{E}
$$

where $\mathrm{Q}$ is the co-ordinate of a molecular vibration. Eq. 2.1 can be simplified to one dimension 


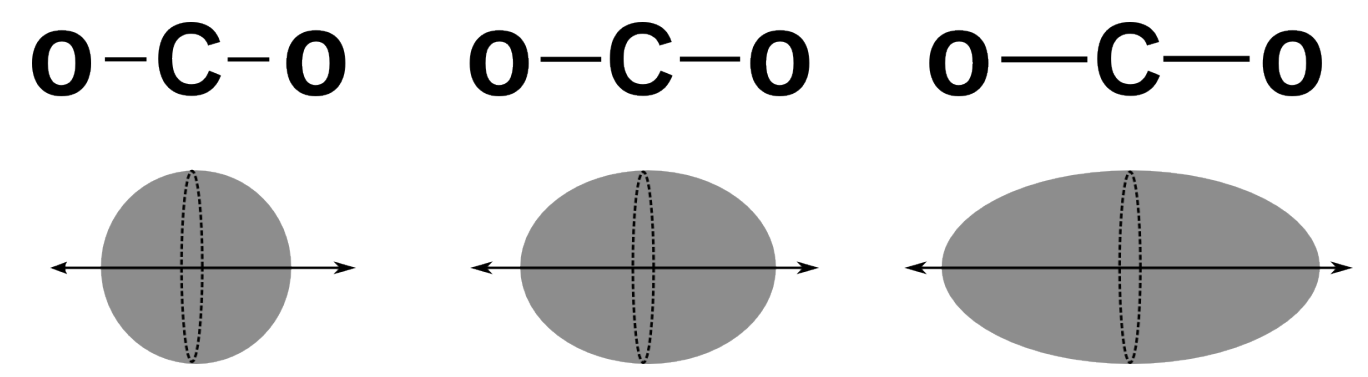

Figure 2.1: The symmetrical stretching mode of $\mathrm{C}_{2}$ is Raman active because the polarisability (depicted in grey) changes with the vibration

using $\mathrm{E}_{x}=E_{x}^{0} \cos \left(2 \pi \nu_{0}\right) t$ and $\mathrm{Q}_{x}=Q_{x}^{0} \cos \left(2 \pi \nu_{i}\right) t$, where $\nu_{0}$ and $\nu_{i}$ are the photon and phonon frequencies respectively. Eq. 2.1 can now be rewritten as:

$$
\mathbf{P}=\alpha E_{x}=E_{x}^{0} \cos \left(2 \pi \nu_{0}\right) t+\frac{\delta \alpha}{\delta Q} Q \quad E_{x}^{0} \cos \left(2 \pi \nu_{0}\right) t Q_{x}^{0} \cos \left(2 \pi \nu_{i}\right) t
$$

Eq. 2.2 can be further simplified using the trigonometric identity:

$\cos \theta_{1} \cos \theta_{2}=\frac{1}{2}\left(\cos \left(\theta_{1}-\theta_{2}\right)+\cos \left(\theta_{1}+\theta_{2}\right)\right)$.

$$
\begin{aligned}
\mathbf{P}= & E_{x}^{0} \cos \left(2 \pi \nu_{0}\right) t \\
& +\frac{1}{2} \frac{\delta \alpha}{\delta Q} Q \quad E_{x}^{0} Q_{x}^{0} \cos \left(2 \pi\left(\nu_{0}-\nu_{i}\right) t\right) \\
& +\frac{1}{2} \frac{\delta \alpha}{\delta Q} Q \quad E_{x}^{0} Q_{x}^{0} \cos \left(2 \pi\left(\nu_{0}+\nu_{i}\right) t\right)
\end{aligned}
$$

Eq. 2.3 describes elastic scattering where the photon survives a collision with a molecule without any change in energy. The remaining two terms depict the two photon-phonon interactions of the Raman effect. The first is the Stokes effect where the photon loses energy to a phonon (Eq. 2.4). The second is the inverse effect where the photon gains energy from a phonon and is known as the Anti-Stokes effect (Eq. 2.5). Most molecular signatures only rely on the Stokes effect however, more sophisticated molecular analyses use both these effects.

Inelastic collisions are rare and the Raman signal is very weak - one of the reasons it lay undiscovered until the 1930s. The most widely used method for signal amplification is the tuning of the laser energy to match an electronic transition. This results in a signal several orders of magnitude greater $\left(10^{2}-10^{4}\right)$ which can be reliably isolated by simple optics to give clean spectra with high signal-to-noise ratios. This matching of the laser energy to an electronic transition is considered a resonance process and typically called 'Resonance Raman Spectroscopy' or RRS. 
The strength of the Raman signal is strongly dependent on a number of material characteristics including the polarisability of the molecule. For example, molecules with delocalised $\pi$ orbitals that can be easily distorted usually display strong Raman signals. Sp ${ }^{2}$ hybridised C-C bonds of SWNTs have a delocalised $\pi$-electron cloud that can be perturbed by molecular vibrations. In SWNTs, the strong Raman signature is further amplified by the resonant Raman effect when the incoming laser photon matches an electronic transition in the material. The energy differences between the electronic density of states in SWNTs are clearly-defined electronic transitions $\left(\mathrm{E}_{i i}\right)$ that can be matched with lasers in the UV-NIR range. More details of SWNT Raman modes are given in Chapter 5.2 .

\subsubsection{Temperature dependence}

Phonon frequencies in crystalline materials can be calculated on the basis of a harmonic potential. The spring constant(s) connecting the masses are assumed to be constant and independent of the distance between the masses. However, both these features are simplistic assumptions and are only applicable in certain situations. Deviations from these ideal situations result in 'anharmonicity'.

\section{Peak frequency}

Typical lattice potentials are only harmonic at low temperatures (small atomic displacements). The potential moves away from the harmonic well at higher temperatures due to greater atomic displacements; requiring higher order cubic and quartic terms to describe the potential well. This deviation in turn affects atomic spacings and the cell volume. The cell volume can also be altered by external pressure. According to the Grüneisen model, a change in cell volume $(V)$ can be shown to affect the phonon frequency. These changes are related via the volume Grüneisen parameter, $\gamma_{V}[\underline{6}$ :

$$
\gamma_{V}=\frac{\beta}{\chi C_{V}}
$$

The parameters, $\beta, \chi$ and $C_{V}$, stand for the volume thermal expansion coefficient, volume compressibility and heat capacity. Eq. 2.6 is assumed to be constant and independent of temperature. This assumption was refuted by early temperature-dependent studies of the Raman mode of diamond [7] which clearly showed a decrease in the $T_{2 g}$ Raman mode of diamond with temperature. Thus, for any vibrational mode $\left(\omega_{i}\right)$, a mode Grüneisen parameter, $\gamma_{i}$, is defined as [6]:

$$
\gamma_{i}=-\frac{d \ln \omega_{i}}{d \ln V}=\frac{B(0)}{\omega_{0}} \frac{d \omega}{d P}
$$


The pressure dependence of the Raman mode, $\frac{d \omega}{d P}$, is used to calculate $\gamma_{i}$ given the bulk modulus $(B(0))$ and the natural frequency of the mode: $\omega_{0}$. Since the volume change due to temperature can be calculated using the linear thermal expansion coefficient $(\alpha)$, the change to the vibrational frequency (due to volume effects alone) can be written as:

$$
\begin{aligned}
\Delta_{1}(T) & =\omega_{0} \exp \left(-3 \gamma_{i}{ }^{Z} \quad \beta\left(T^{\prime}\right) d T^{\prime}\right) \\
& =\omega_{0} \exp \left(-3 \gamma_{i} \alpha T\right)
\end{aligned}
$$

Eq. 2.8 is the approximation for a temperature-independent thermal expansion coefficient. The parameter, $\omega_{0}$, is the natural/limiting phonon frequency as $T \rightarrow 0 \mathrm{~K}$. The pre-factor of ' 3 ' converts the linear thermal expansion into a volume thermal expansion coefficient. However, Eq. 2.8 ignores the so-called explicit contribution of anharmonicity arising from phonon-phonon interactions. One of the simplest cases of phonon-phonon interactions - most relevant to Raman spectroscopy - is the decay of the optical phonon into two acoustic phonons [8]. $\Delta_{2}(T)$ can be written as shown in Eq. 2.9 and describes the contribution from the decay of an optical phonon into two identical phonons with frequency $\omega_{0} / 2$ and opposite momenta (the so-called 'three phonon' process). The thermal occupation of these daughter phonons is given by the Planck distribution: $\left(\exp \left(h c \omega_{0} / 2 k_{B} T\right)-1\right)^{-1}$. The parameter, $A_{\omega}$, is an independent fitting parameter. It is worth noting here the slight change in the representation of the phonon energy from the typical $\hbar \omega_{0}$ to $h c \omega_{0}$, where $c$ and $\omega_{0}$ are in units of $\mathrm{cm} / \mathrm{s}$ and $\mathrm{cm}^{-1}$ respectively.

$$
\Delta_{1}(T)=A_{\omega} \quad 1+\frac{2}{\exp \frac{h c \omega_{0}}{2 k_{B} T}-1}
$$

Thus, the total change in the vibrational frequency due to temperature, is a sum of the implicit or, volume contribution and, the explicit phonon-phonon interaction term is given by Eq. 2.10.

$$
\omega(T)=\Delta_{1}(T)+\Delta_{2}(T)
$$




\section{Linewidth}

Lineshapes have a significant meaning in spectroscopy as they are derived from the microscopic picture of excitation and relaxation of electronic transitions. The characteristic spectral lineshape of spectroscopy is a Lorentzian from the decay transition of a molecule from its excited to ground state. Mathematically, this process can be described by a damped harmonic oscillator. The 'damping' aspect is effectively a decay back to the ground state in the time domain. This decay appears as a Lorentzian in the frequency domain [9]. Hence, the Raman linewidth, $\Gamma$, is proportional to the inverse phonon lifetime, $\tau_{p h}$. The phonon lifetime can be calculated using the relation: $1 /(\pi c \Gamma)$. Since the lifetime of the excited phonon is related to its decay into lower energy phonons, $\Gamma(T)$ can be modelled using the equivalent of Eq. 2.9 shown in Eq. 2.11 where only the independent fitting parameter changes from $A_{\omega}$ to $A_{\Gamma}$.

$$
\begin{gathered}
\Gamma(T)=A_{\Gamma} \quad 1+\frac{2}{\exp \frac{h c \omega_{0}}{2 k_{B} T}-1} \\
\Gamma(T)=A_{\Gamma} \quad 1+\frac{2}{\exp \frac{h c \omega_{0}}{2 k_{B} T}-1}+B_{\Gamma} \quad 1+\frac{3}{\exp \frac{\hbar \omega_{0}}{3 k_{B} T}-1}+\frac{3}{\exp \frac{\hbar \omega_{0}}{3 k_{B} T}-1{ }^{2}}
\end{gathered}
$$

The three-phonon decay process is not the only possible phonon decay process. Higher order processes where the optical phonon decays into more than two phonons are also possible. The functional form of the Klemens process given in Eqs. 2.11 and 2.9 is effectively linear at high temperatures. However, there are several materials whose linewidth has a distinct curvature at high temperatures [10, 11]. For example, $\Gamma(T)$ in silicon becomes non-linear above $\sim 600 \mathrm{~K}$ (top plot in Figure 2.2a) [11]. The data was digitised from the plots in the original paper and replotted in Figure 2.2. In this case, the authors' extended the model of Klemens to include a 'four-phonon' decay process where the optical phonon decays into three daughter phonons of frequency $\omega_{0} / 3$ with a total momentum of zero. This process is given by the second term in Eq. 2.12. The solid lines in Figure 2.2a show the fit using both Eq. 2.12 (solid red line) and the model proposed by Eq. 2.11 (dotted black line). The values of the fitting constants were obtained from the original paper. It is of note that in materials with a non-linear trend for the position shift alone, the combination of the three phonon process with thermal expansion provides a much better representation of the experimental data [12, 13].

Given the similarity between the Eqs. 2.9 and 2.11, we examine the relationship between $\omega(T)$ and $\Gamma(T)$ when phonon-phonon interactions alone dominate the anharmonicity. Typically covalent materials have small expansion coefficients and phonon decay processes dominates in these materials [14]. Hence, the same silicon data from Balkanski et al. is used to qualitatively understand the aforementioned relationship. We can plot $\omega_{\text {peak }}$ against $\Gamma$ as shown in 

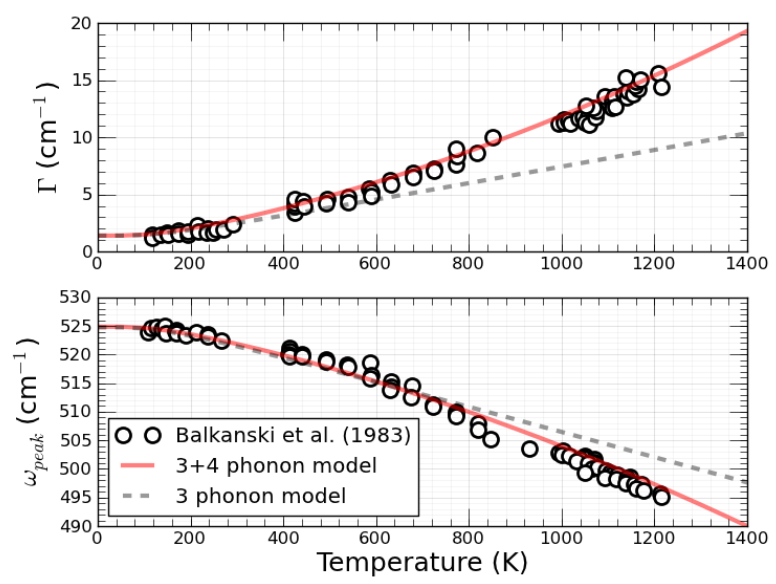

(a) Temperature dependence

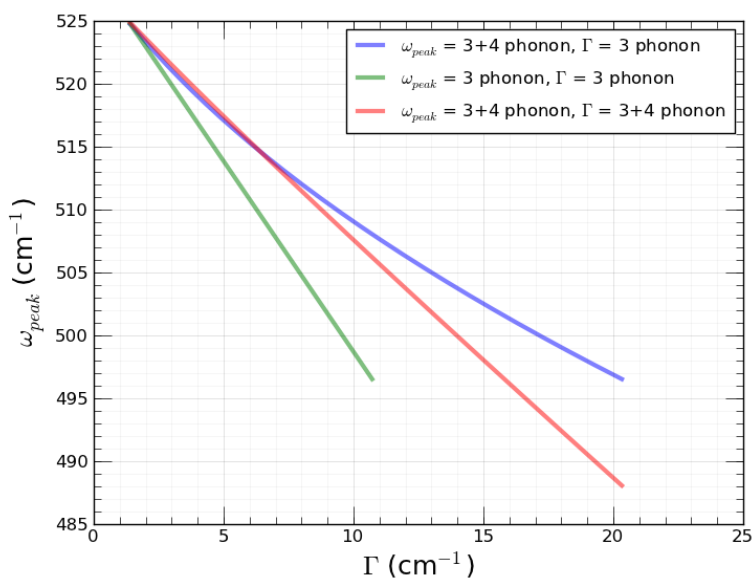

(b) $\omega_{\text {peak }}$ vs. $\Gamma$

Figure 2.2: Temperature dependence in silicon. Data from [11].

Figure 2.2b. This plot shows that a linear relationship between $\omega_{\text {peak }}$ and $\Gamma$ is most likely when they both follow the same decay processes (regardless of the magnitude of the fitting constants).

For the Klemens process, the linearity in the data, above a characteristic temperature, arrives from the high temperature limit of the Planck distribution. Above a certain temperature (when $2 k_{B} T>h c \omega_{0}$ ), the exponential can be approximated with the first order Taylor expansion and becomes proportional to $T$. Thus, when the peak position and linewidth are linear functions of temperature, they can be replotted to give a linear trend between each other. When the two parameters follow a different process, the relationship between the parameters is no longer linear. This representation allows for quick visual examination and estimation of decay mechanisms in the position shift and linewidth.

\section{Intensity}

The ratio of the anti-Stokes $\left(I_{A S}\right)$ and Stokes $(I S)$ intensities is a function of temperature. Eq. 2.13 gives this ratio for the non-resonant Raman case [15]. This equation is altered slightly the original in the cited reference. The parameter, $\omega_{0}$, is used instead of $\omega(T)$. We can do this because $\omega_{\text {laser }}$ is much greater than $\omega(T)$. Both Raman studies on cupric oxide and SWNT networks have focused on the low wavenumber region below $300 \mathrm{~cm}^{-1}$. For this region, $\omega$ is less than $2 \%$ of $\omega_{\text {laser }}$ (for $E_{\text {laser }}$ of $1.96 \mathrm{eV}$ ). The second addition is that of a correction factor: $\gamma$. This correction factor accounts for different detection efficiencies for Stokes and anti-Stokes photons, laser heating effects [16] or different scattering rates for Stokes and anti-Stokes photons [17]. 


$$
\frac{I_{A S}}{I_{S}}=\gamma \frac{\omega_{\text {laser }}-\omega_{0}}{\omega_{\text {laser }}+\omega_{0}} \quad \exp \frac{-h c \omega_{0}}{k_{B} T}
$$

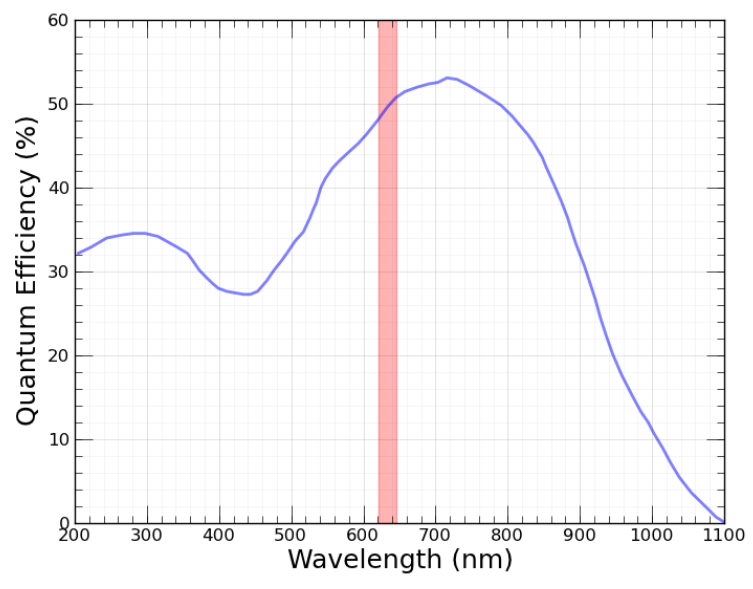

(a) Detection efficiency

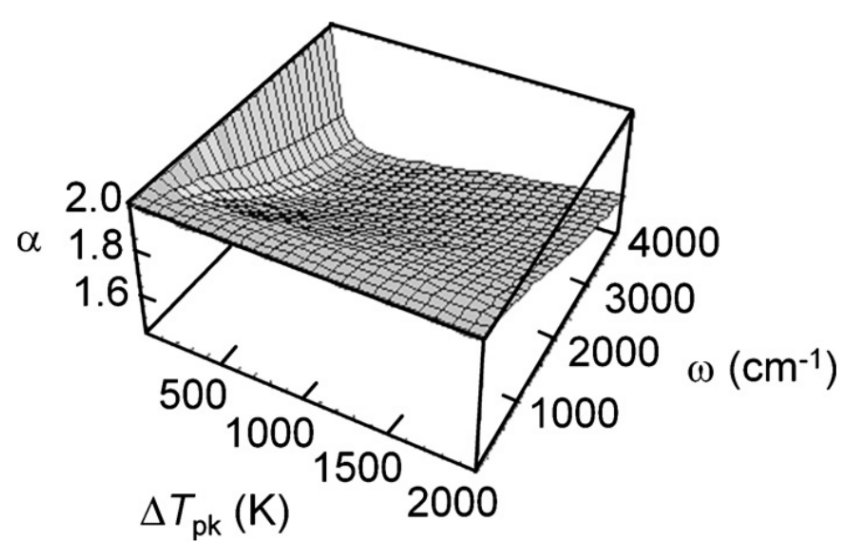

(b) Effect of spatial temperature on $\gamma$

Figure 2.3: (a) Detection efficiency of the Symphony CCD detector attached to the LabRam. The highlighted red region gives the limits for the Stokes and anti-Stokes photons in $\mathrm{CuO}$. (b) Effect of spatial temperature on $\gamma$. In this plot, $\alpha$ is equivalent to $\gamma$. Figure reproduced from [16].

The detection efficiency of the Symphony II CCD detector used in our experiments is shown in Figure 2.3a. The Stokes and anti-Stokes photons from $\mathrm{CuO}$ are given by the limits of the red region. The difference in efficiency is barely $3 \%$ and is unlikely to affect $\gamma$. Another effect that can change the value of $\gamma$ from 1.0 is laser-induced temperature distributions in the sample. According to Herman [16], the correction factor for materials that are heated inhomogeneously by a gaussian laser depends on both the phonon frequency and the peak temperature of the induced distribution. Figure $2.3 \mathrm{~b}$ shows that Raman modes with a frequency below 500 $\mathrm{cm}^{-1}$ require a constant correction factor of $\sim 2.0$ regardless of the induced peak temperature. However, measuring the temperature from the anti-Stokes/Stokes ratio for phonon frequencies above $500 \mathrm{~cm}^{-1}$ is highly dependent upon the peak temperature.

Eqs. 2.14 and 2.15 give the individual temperature-dependent intensities of the Stokes and anti-Stokes peaks [15]. Both functions increase with temperature. However, there are several cases where the Stokes intensity is a decreasing function of temperature - due to changes in the inter-atomic distances - which has been shown to affect the Raman cross-section [18] or, changes in the material's optical properties [17]. 


$$
\begin{aligned}
I_{S} & \propto \frac{\hbar}{2 \omega_{0}}\left(\omega_{\text {laser }}-\omega_{0}\right)^{4} 1+\left(\exp \left(-h c \omega_{0} / k_{B} T\right)-1\right)^{-1} \\
& \propto 1+\left(\exp \left(-h c \omega_{0} / k_{B} T\right)-1\right)^{-1} \\
& I_{A S} \propto \frac{\hbar}{2 \omega_{0}}\left(\omega_{\text {laser }}+\omega_{0}\right)^{4}\left(\exp \left(-h c \omega_{0} / k_{B} T\right)-1\right)^{-1}
\end{aligned}
$$

The integrated intensity (or signal) of a given Raman mode is proportional to the observed scattering rate: the number of Raman-scattered photons that leave the sample. Compaan and Trodahl [17 derive a relation for this property using the material's optical properties (e.g. absorption and refractive index) in addition to experimental parameters like the solid angle of the objective. The authors' measure the Raman signal (Figure 2.4a) and subsequently calculate the scattering rate (Figure 2.4b) as a function of both temperature and laser energy. Both these figures show the decrease in Raman-scattered photons from silicon with increasing temperature. The scattering rate shows a varying trend due to the sensitivity of the Raman signal near an electronic transition; in this case, the direct bandgap of silicon at $3.32 \mathrm{eV}(373 \mathrm{~nm})$. Combined with an earlier detailed study [19], Compaan and Trodahl assign the decreases in the Raman signal to changes in the optical constants (refractive index and optical transmission) of silicon with temperature.

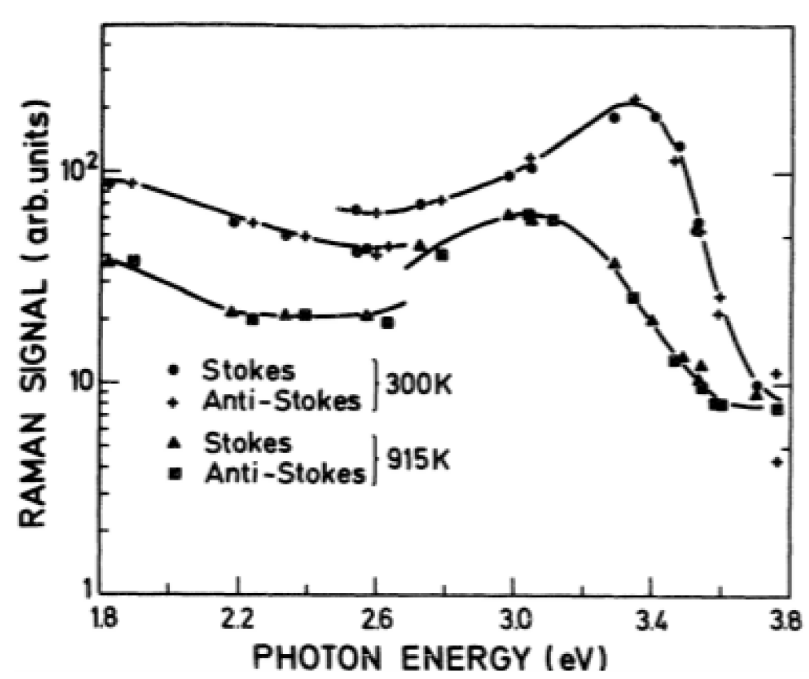

(a) Raman signal

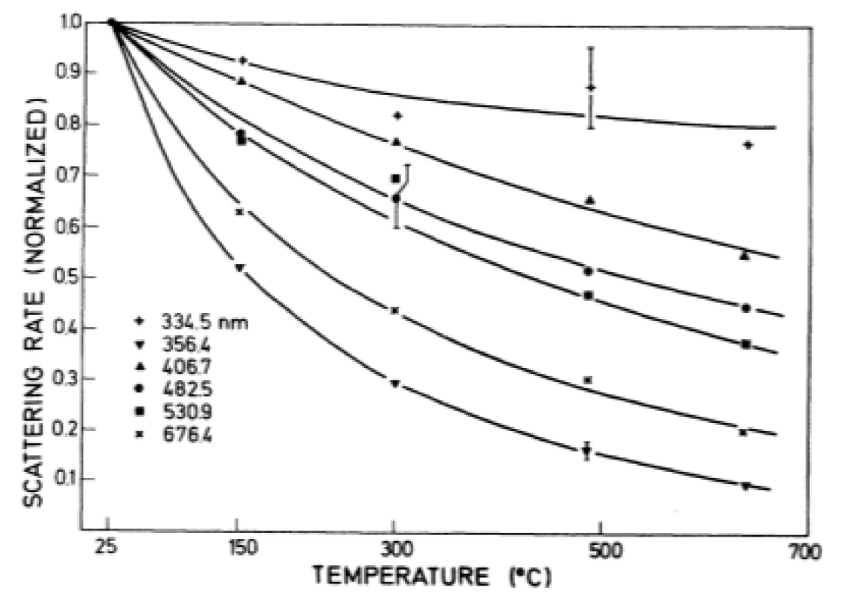

(b) Scattering rate

Figure 2.4: Scattering rate and Raman signal as a function of temperature and laser energy. Figures from [17] 


\subsubsection{Temperature profiles induced by gaussian beam}

The calculation of the spatial temperature distribution in a sample due to a laser beam with a gaussian intensity distribution in the plane of focus (see Eq. 2.19 and Figure 2.8a) was first done numerically by Lax [20]. Lax defines the spatial distribution of temperature, N(r,z,w), as a function of $\mathrm{R}, \mathrm{Z}$ and $\mathrm{W}$ are the normalised spatial distance, depth into the sample and spread of the gaussian beam respectively. The explicit definitions of these parameters are given as: $\mathrm{R}=r / \omega_{0}, \mathrm{Z}=z / \omega_{0}$ and $\mathrm{W}=\alpha \omega_{0}$ where $\alpha$ and $\omega_{0}$ are the absorption coefficient and the beam waist respectively (see Figure 2.7). The absorption coefficient of different forms of $\mathrm{CuO}$ [21, 22, 23] are given in Figure 2.5a.

In the limit of $\mathrm{W} \rightarrow \infty$ (at large values of $\alpha), N(R, Z, W)$ is given by Eq. 2.16.

$$
N(R, Z, W)=B \underbrace{Z_{\infty}}_{0} d \lambda J_{0}(\lambda R) F(R) \times \frac{W \exp (-\lambda Z)-\lambda \exp (-W Z)}{W^{2}-\lambda^{2}}
$$

$B=\alpha P / 2 \pi K F(0)$ where $P$ and $K$ are the laser power and thermal conductivity respectively) and $F(\lambda)=\frac{1}{2} \exp \left(-\frac{1}{4} \lambda^{2}\right) . F(\lambda)$ is the Hankel transform of $\left.f(R)\right)$. The Hankel transform allows the transformation from a double integral into a one dimensional integral when the function is radially symmetric [20]. $J_{0}(x)$ is the zeroth order Bessel function.

The calculation of $N(R, Z, W)$, shown in Figure 2.5b, was done numerically using the quadrature integration package of Scipy. The zeroth order Bessel function $J_{0}(x)$ was computed using the predefined function, jn, from scipy.special. Figure 2.5b shows that decreasing the value of $W$ makes the curve shallower. The value of $W$ can be changed by either decreasing the absorption coefficient or decreasing the beam spot. It is important to note here that the above expressions and curves for the temperature distribution are those for the focal plane. The green line in this curve corresponds to $\alpha \sim 1 \mathrm{e} 5 \mathrm{~cm}^{-1}$ and $\omega_{0}=600 \mathrm{~nm}$ - which are the absorption coefficient of $\mathrm{CuO}$ at $\sim 2 \mathrm{eV}$ and the beam waist of the $1.96 \mathrm{eV}$ laser in our experimental setup. This curve shows that the temperature distribution decreases to $10 \%$ of the value at the peak within $\sim 6 \mu \mathrm{m}$. This spot radius, which is well within the window of the collection optics: $\sim 10 \mu \mathrm{m}$ radius (Section 2.2.5), indicates that our chosen optics can pick up the full extent of the spatial temperature distribution induced by a gaussian laser.

\subsection{Raman spectroscopy: experimental aspects}

Raman spectra were collected using a Horiba Jobin-Yvon LabRam high resolution (HR) Raman spectrometer. The following section goes through the characteristics of the instrument, 


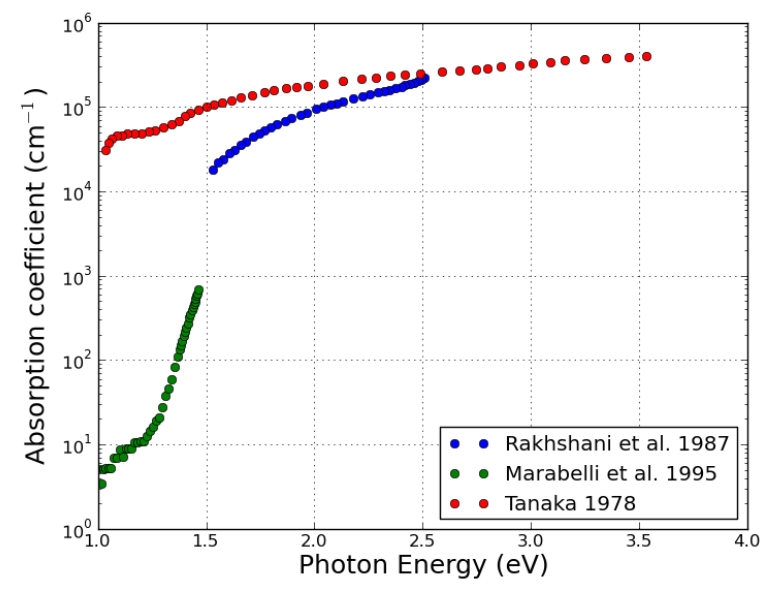

(a) $\mathrm{CuO}$ absorption

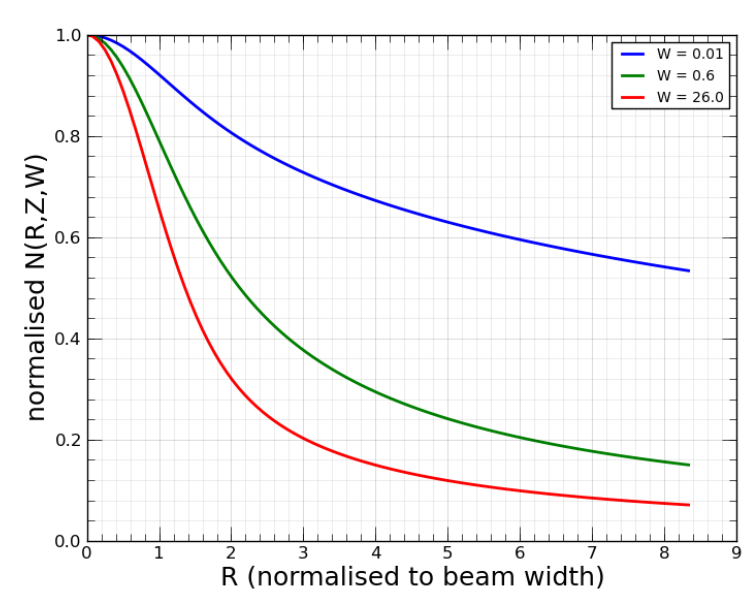

(b) Temperature distribution

Figure 2.5: (a) Absorption coeffiecient of $\mathrm{CuO}$ as a function of energy from three different papers. (b) Closed form of $\mathrm{N}(\mathrm{R}, \mathrm{Z}, \mathrm{W})$ as given by Lax [20]. N.B. all these curves are for $\mathrm{Z}=0$.

the experimental parameters and the reasons for these parameters in detail.

\subsubsection{LabRam optics}

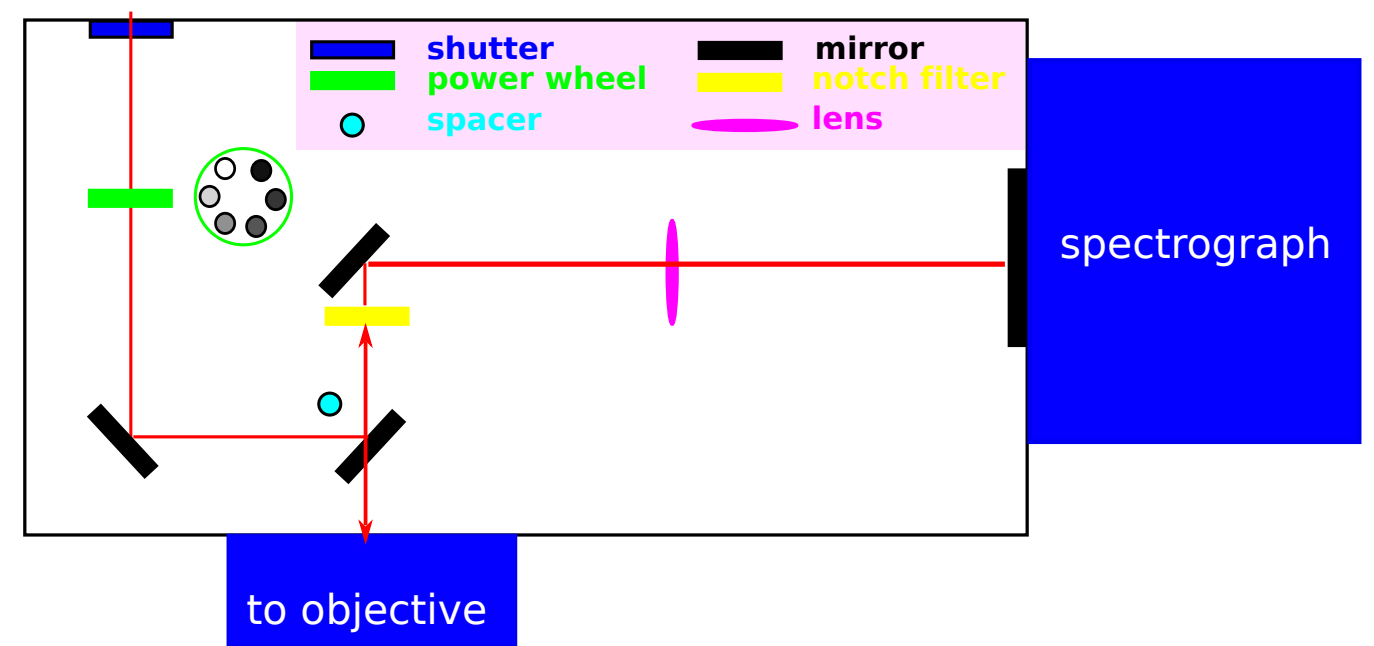

Figure 2.6: Optics set up for the LabRam confocal microscope.

Figure 2.11 shows the major components of the spectrometer along the beam path. From the shutter, the laser beam passes through a power wheel (a collection of netural density filters) before reaching the objective. The Raman-scattered light from the sample passes through the notch filter while the Rayleigh-scattered light is reflected with more than $90 \%$ efficiency. A small stop called a 'spacer' adjusts the angle of the beam onto the notch filter. As the notch filter ages, the spacer is changed so that the beam falls on a less worn part of the filter. The Raman-scattered light then travels to the spectrograph via a confocal pinhole. 


\subsubsection{Gaussian laser beams}

Laser beams coming from lab laser sources are typically modelled as gaussian beams. This allows the intensity distribution in the transverse and propagation directions to be modelled given the beam waist and the laser wavelength. The beam waist for a $632.8 \mathrm{~nm}$ laser using the LabRam set up and a $100 \times 0.9$ N.A objective was calculated using the 'slit size dependence method' as described in the supporting information of [24]. The term 'slit' is equivalent to pinhole.

The beam waist, $\omega_{0}$, for a misaligned beam is calculated using Eq. 2.17. This reduces to Eq. 2.18 for perfect alignment. Given that we are using a real system that is usually not at perfect alignment, Eq 2.17 was used. The parameters $L, W_{0} x_{c}$ and $y_{c}$ represent the slit halflength (assuming a square slit), the beam waist $\left(\omega_{0}\right)$ multiplied by a magnification factor $(X$ where $X=56$ for a $100 \times$ objective), and misalignment in the $x$ and $y$ directions respectively. The magnification factor $X$ results because the confocal optical set up has 'scaling' factor of 0.56 [24]. So, the total magnification of the spot for a $100 \times$ objective is $0.56 \times 100,56$.

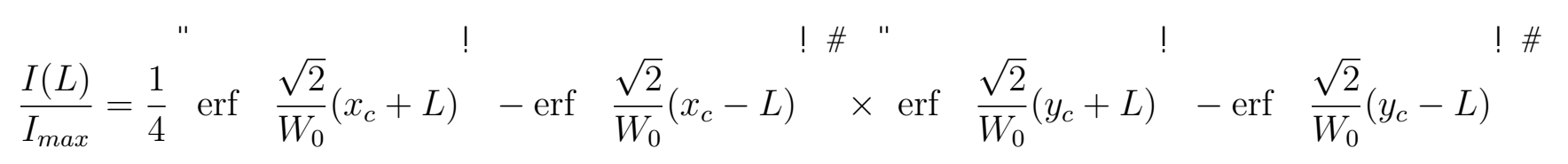

$$
\frac{I(L)}{I_{\max }}=\operatorname{erf} \frac{\sqrt{2} L}{W_{0}}
$$

It should be noted that the slit half length $(L)$ is calculated using a simple relation $L=$ $P{ }^{\mathrm{p}} \overline{(8)}$ where $P$ is the diagonal length of the confocal pinhole. The relation comes about from the Pythagorous theorem for a square aperture with sides $2 L$ and a diagonal length $P$. The value of the confocal pinhole can be varied from $\sim 100$ to $1000 \mu \mathrm{m}$.

The beam waist was calculated using the slit size dependence of the silicon Raman peak at $520 \mathrm{~cm}^{-1}$. The results are shown in Figure 2.7. The possible range of values for $\omega_{0}$ were calculated as those between $x_{c}=y_{c}$ and $x_{c} \neq y_{c}$. From these two fitted values, the average beam waist is calculated to as $\sim 600 \mathrm{~nm}$. We can now model the intensity distribution of the laser beam in the transverse $(x)$ and propagation $(z)$ directions (Figure 2.8a) using the formula in Eq. 2.19, Figure 2.8b shows the beam is quite tight in the transverse direction. The intensity tapers off within a radius of $1 \mu \mathrm{m}$. The laser intensity has a larger spread along the propagation direction and is described by a lorentzian shape (Figure 2.8c). 


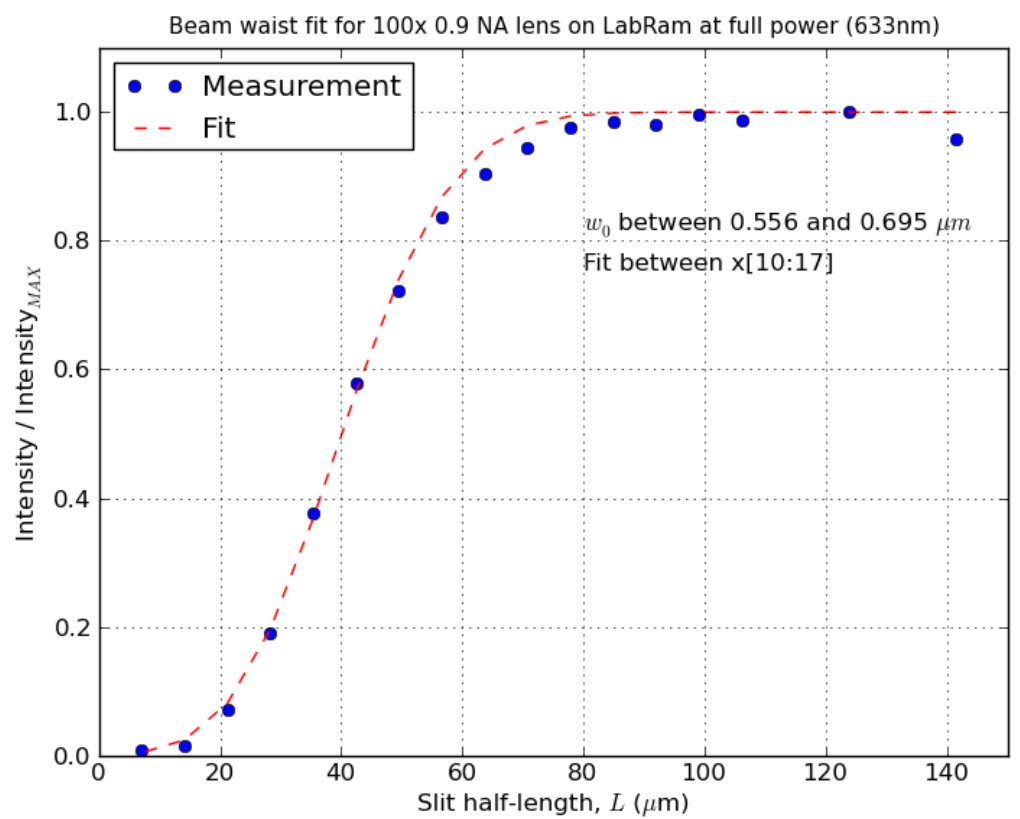

Figure 2.7: Beam waist measurement for $632.8 \mathrm{~nm}$ laser with the $100 \times 0.9$ N.A. objective.

$$
I(x, z)=I_{0} \quad \frac{\omega_{0}}{\omega(z)} \quad \exp \frac{-2 x^{2}}{\omega(z)^{2}}
$$

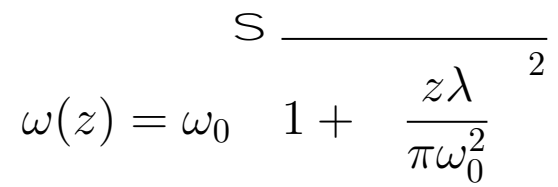
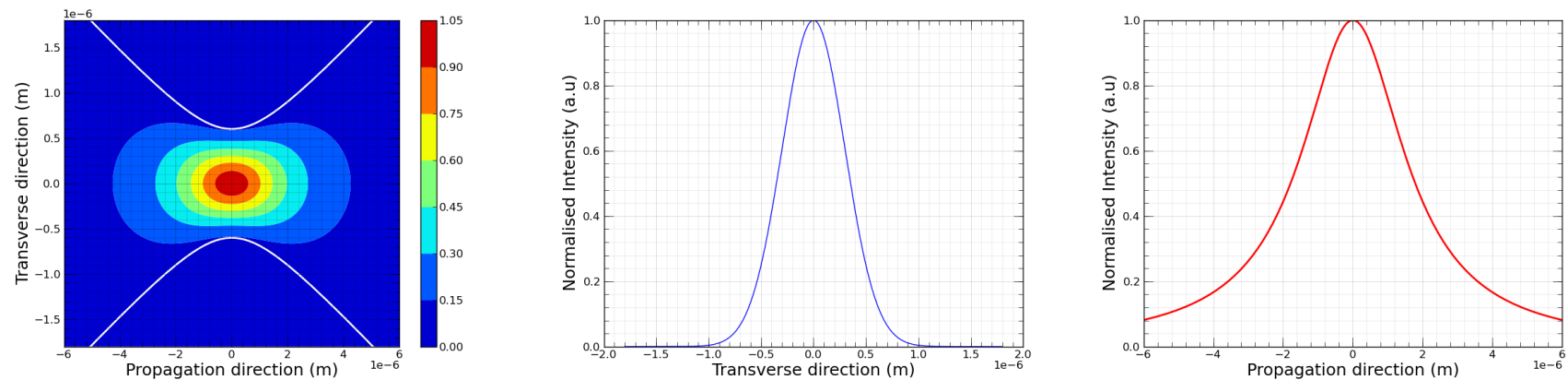

(a) $2 \mathrm{D}$ representation of the gaussian(b) Intensity in the transverse direction(c) Intensity in the propagation direcbeam tion

Figure 2.8: Intensity distribution for $632.8 \mathrm{~nm}$ laser and a beam waist of $600 \mathrm{~nm}$ in $2 \mathrm{D}$ and cross-sections in the transverse and propagation directions. 


\subsubsection{Laser power}

The laser beam passes through a power wheel after coming through the main shutter. Slots around the outside of the wheel contain neutral density filters to modulate the laser power (green wheel in Figure 2.11). The laser power without any filters is between 5 and $6.7 \mathrm{~mW}$. The fractional transmittance of the filters is defined as: $I / I_{\max }=10^{-D}$. Thus, the left hand side of that relation is $\sim 51 \%$ when $D=0.3$.

\begin{tabular}{|c|c|}
\hline Filter name & Fractional transmittance \\
\hline \hline D0.3 & $51 \%$ \\
\hline D0.6 & $27 \%$ \\
\hline D1 & $10 \%$ \\
\hline D2 & $1 \%$ \\
\hline \hline
\end{tabular}

Table 2.1: Neutral density filter specifications.

\subsubsection{Notch filter and spacer}

As discussed in Section 2.1, the majority of the laser light is elastically scattered. As only a small fraction of laser light from the sample comes from Raman scattering, the bulk of the light from the sample needs to be filtered. One way of filtering Rayleigh-scattered light is by using a notch filter. Notch filters have a preferentially large reflectivity for a narrow wavelength band around the laser wavelength. A small screw called a 'spacer' adjusts the angle of the half-mirror which lets through light from the sample onto the notch filter. This angle is adjusted by the size of the spacer as shown in Figure 2.9a. Typically, the spectrometer is aligned (for a given laser) to a particular spacer size. The $633 \mathrm{~nm}$ is aligned with the \#6 spacer in place. Figure 2.9b shows the variation in the Raman signal when the spacer is changed. Several characteristics of the Raman mode change: the peak position, linewidth and even the lineshape. Thus, the right spacer is critical for any study where analysis of the lineshape components is important.

\subsubsection{Objective}

The $100 \times$ objective with a numerical aperture (N.A.) of 0.9 , was used for all the experiments. This objective has a solid angle of 3.54 sr or ( $28 \%$ of the total solid angle). The solid angle $(\Omega)$ is defined as the two-dimensional angle in three-dimensional space that an object subtends at a point. The solid angle of an arbitrary shape is given by Eq. 2.21 while Eq. 2.22 gives the solid angle for a cone. The value of $\theta$ is determined using the equation $N . A=n_{m} \sin (\theta)$ where $n_{m}$ is the refractive index of the medium the objective is immersed in. 


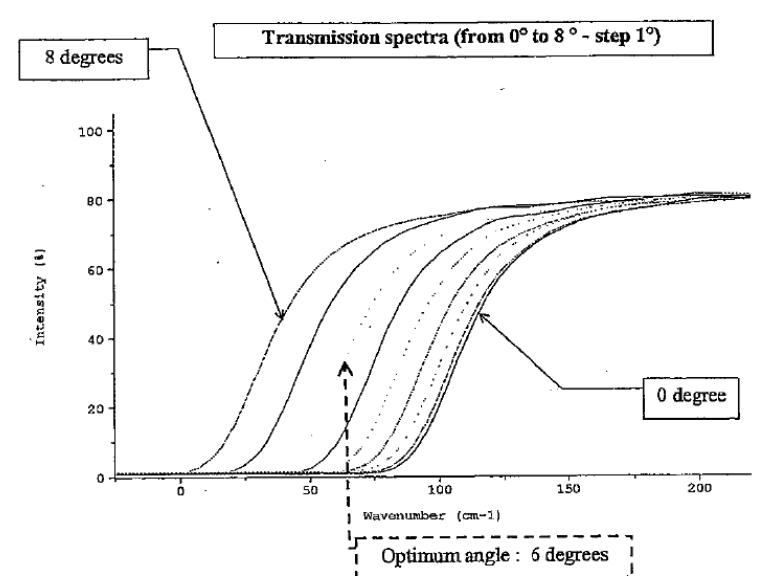

(a) Spacer number

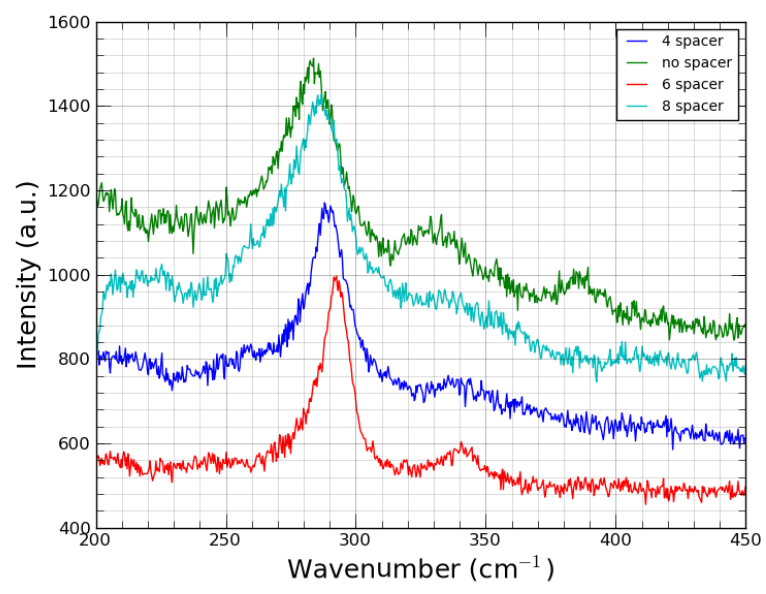

(b) Effect of spacer

Figure 2.9: (a) Cut off intensity as a function of wavenumber (figure from the LabRam manual). (b) Stokes Raman spectra of $\mathrm{CuO}$ thin film (using $100 \times 0.6$ N.A objective) as a function of spacer number.

$$
\begin{aligned}
\Omega & =\mathbf{Z}_{2 \pi} \mathbf{Z}_{\theta} \sin \left(\theta^{\prime}\right) d \theta^{\prime} d \phi \\
\Omega_{\text {cone }} & ={ }_{0}^{0}{ }^{0}[1-\cos (\theta)]
\end{aligned}
$$

The objective is the major component of the collection optics that determine the effective radius from which light is collected. For a fully open pinhole with a 'diameter' of $1000 \mu \mathrm{m}$, the collection area radius is $500 \mu \mathrm{m} / 56$ (magnification factor for 100x lens and second lens in the LabRam collection optics) $\sim 10 \mu \mathrm{m}$. This collection radius is much larger than the $1 \mu \mathrm{m}$ decay radius for the gaussian beam (see Figure 2.8b).

\subsubsection{Spectograph}

Inside the spectograph, a dispersive grating spreads the Raman-scattered light across a CCD detector (1024 pixels wide). The choice of grating affects the resolution of the collected data as well as the spectrum. We have consistently used the high resolution 1800 lines/mm grating for all our experiments. It should be noted that the Raman intensity is affected by the choice of grating. For example, the silicon $520 \mathrm{~cm}^{-1}$ signal is higher by a factor of $\sim 3$ with the 600 lines/mm grating since this grating is blazed at the laser wavelength of $632.8 \mathrm{~nm}$. However, Raman signal from both $\mathrm{CuO}$ and SWNT networks were more than adequate with the 1800 lines/mm grating.

The CCD, or charge-coupled device, is a silicon detector cooled to liquid nitrogen temperatures for optimum efficiency. All data reported here were collected with a Symphony II CCD 
detector attached to the LabRam spectrometer.

\subsubsection{Confocality}

The LabRam is considered a confocal Raman spectrometer. A confocal spectrometer uses an adjustable pinhole to focus on different 'slices' within the sample. However, a proper confocal microscope has two pinholes: the first pinhole is used as a spatial filter and the second pinhole tunes the axial resolution. The axial resolution works on the principle of only allowing light from the chosen slice in the $z$ direction. Good confocal microscopes can select signal from a thin axial slice of the sample. The optical setup of the confocal microscope for the LabRam (Figure 2.11) only has one pinhole and can only control the axial slicing. The depth of the slice is known as the confocal depth and can be measured from the axial dependence of a given Raman mode.

Raman spectra from silicon were collected for a series of different $z$ values; between \pm 35 $\mu \mathrm{m}$ from the focal plane. The integrated intensities of the silicon $520 \mathrm{~cm}^{-1}$ peak are plotted as a function of axial displacement in Figure 2.10. In this experiment, it is important to ascertain if the detected signal is due to axial detection efficiency alone or if it is a convolution of the lateral and axial detection efficiencies. The lateral detection limit is determined by $56^{*} \omega(z)$. The magnification factor of 56 gives the dimensions of the laser spot projected on the confocal pinhole. The value of $\omega(z)$ is given by Eq. 2.20. If $56^{*} \omega(z)$ is less than the half length of the operational pinhole size $(1000 \mu \mathrm{m} / 2=500 \mu \mathrm{m})$, the Raman signal is only dependent upon the axial detection efficiency. If $56^{*} \omega(z)$ is greater than $500 \mu \mathrm{m}$, then the Raman signal is a convolution of both axial and lateral detection efficiencies since the projected spot is now larger than the pinhole. The $z$ value at the lateral detection limit is $27 \mu \mathrm{m}$. Hence, the integrated intensity as a function of $z$ represents the axial detection efficiency between $\pm 27 \mu \mathrm{m}$ : most of the curve seen in Figure 2.10 .

The full width at half maximum (FWHM) of the axial detection efficiency gives the confocal depth of the chosen optical set up. In this case, the confocal depth is around $6 \mu \mathrm{m}$. As a small side note, dividing the integrated intensity by the area of the beam spot $\left(\frac{\pi}{2} \omega(z)^{2}\right)$ gives the intensity of the gaussian beam in the propagation direction (Figure 2.10).

\subsubsection{Confocal pinhole effects}

Reproducibility of spectral characteristics are ensured by using a highly specific set of instrumental conditions. Despite these precautions, a poorly aligned beam could corrupt the results. Unfortunately, the quality of the alignment doesn't have a fine grading and can only be assured for a short while after the entire spectrometer has been realigned. A misaligned beam could 


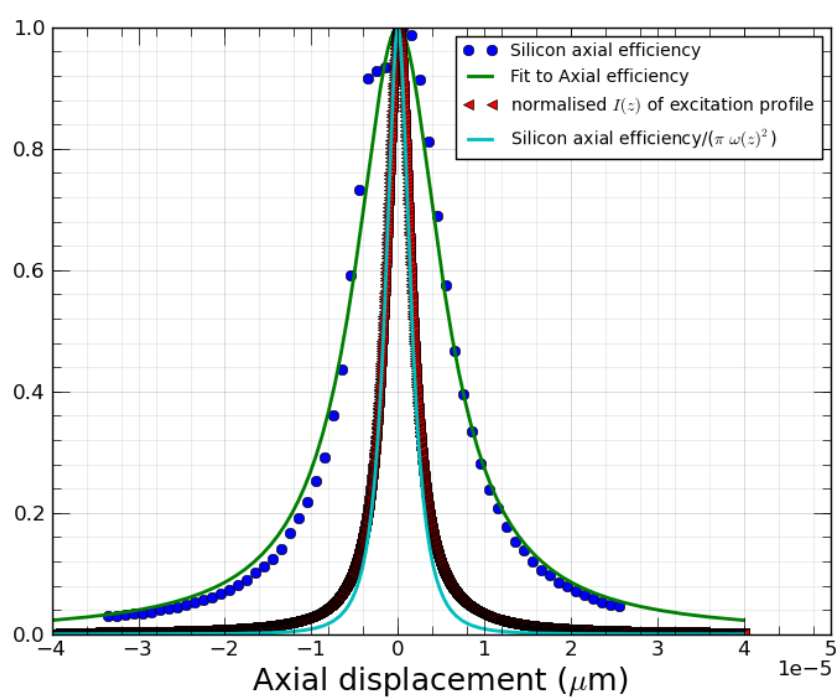

Figure 2.10: Axial detection efficiency of silicon for a fully open pinhole and using the $100 \times 0.9$ NA objective and 1800 (lines/mm) grating.

introduce artefacts like peak shifts as it no longer arrives at the same place on the grating compared to a centrally aligned beam. One route to restricting these artefacts is to constrict the confocal pinhole so that only the center of the beam, which contains straight light rays, goes through. However, this route comes at the price of signal intensity and a small pinhole is only chosen when the beam is considerably misaligned. Chapter 6.3 .3 gives the details of the experiment that determines the quality the beam alignment by comparing spectra taken with different pinholes.

\subsubsection{Measuring temperature-dependent Raman spectra}

Temperature dependence of Raman spectra were carried out using a Linkam THMS 600 cryostat. The temperature was controlled using a Lakeshore temperature controller. Cooling was achieved with either liquid nitrogren (below ambient) or with water cooling for temperatures above ambient. The cryostat window was cooled with a gentle flow of boil-off nitrogen to prevent condensation. Heating/cooling rates were around $10^{\circ} \mathrm{C} /$ minute. The sample was maintained at the set temperature for at least two minutes while finding the right spot re-adjusting the focus. Due to the dimensions of the cryostat, a $50 \times$ long working distance objective was used for temperature dependence studies. Other experimental parameters were kept the same.

\subsection{Conduction: theoretical aspects}

\subsubsection{Percolation theory}

Percolation theory is the mathematical groundwork behind inter-connectivity in random systems. It can be used to model the dependence of macroscopic properties of the random system 


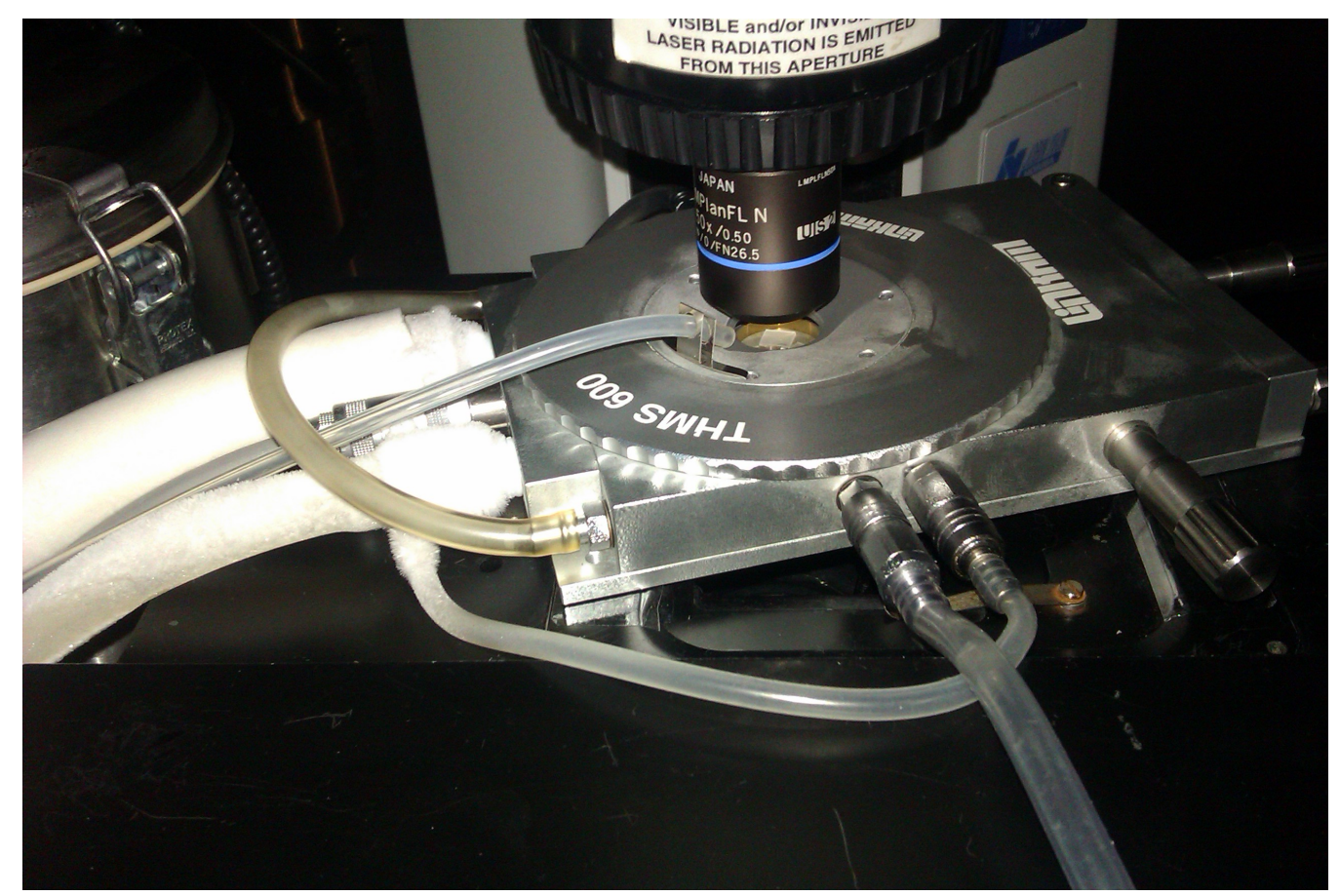

Figure 2.11: Cryostat setup.

in terms of various geometric properties. In the case of conducting networks, the sheet (or volume) conductance can be assessed using parameters like the stick length, width, alignment angle and dimensions of the conduction channel. The percolation model predicts an analytical solution for the conductance of a conducting stick network as:

$$
G=G_{0}\left(A-A_{C}\right)^{\alpha}
$$

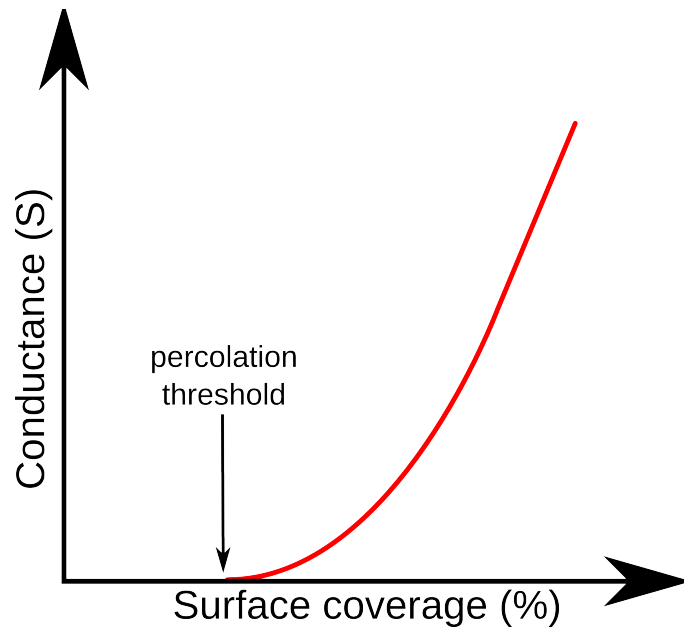

(a) Percolation

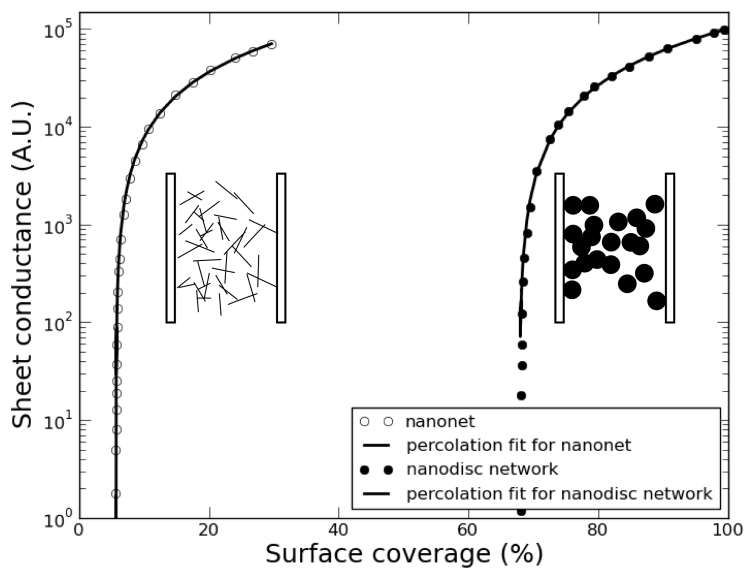

(b) Percolating networks

Figure 2.12: (a) Phase transition in the conductance at the percolation threshold.(b) Percolation thresholds of networks of long wires compared to discs. Figure adapted from [25].

In the case of Eq. 2.23, the conductance is given in terms of the surface coverage, $A . A_{C}$ 
is the percolation threshold, the value of the surface coverage at which long-range connectivity appears. This threshold effectively describes a phase transition as seen in Figure 2.12a. The critical exponent, $\alpha$, is universal across different lattice types and has been evaluated as 1.33 and 1.94 for two and three dimensional percolation respectively [26]. This universality even applies to percolating networks comprised of different components. Fitting the percolation data shown in Figure 2.12b with Eq. 2.23 gives $\alpha$ values of 1.29 and 1.36 for nanonets and nanodiscs respectively. These values are effectively contained within $3 \%$ uncertainty in $\alpha_{2 D}$. Eq. 2.23 only applies near the percolation threshold. When a sufficient number of metallic pathways have been established, the power dependence crosses over to a linear regime [27].

\subsubsection{Conduction mechanisms}

The junction resistance in nanowire networks is the limiting resistance and is particularly pronounced in SWNT networks [28]. This implies that not all connections will be electrically active though most perolation models to date neglect to account for probabilistic transport at nodes. Hence, the need arises to accurately describe conduction at these junctions. This section will briefly outline some of the prevalent theories that describe conduction mechanisms in materials where the junction resistance between conducting regions determines the sheet conductance.

Junctions between any two connected components can be thought of as potential barriers. Both spatial and energetic components contribute to the potential barrier though, in a given random system, the potential barrier has a distribution of values [29]. Tunnelling probabilities through the barrier then become the limiting factor at these nodes. Mathematical models that describe these probabilities can be invoked to explain conduction mechanisms. In the case of energetically and spatially thin barriers separating metallic regions, fluctuation induced tunnelling has been shown to dominate conduction. When barriers are large in both real and energy space, phonon-assisted tunnelling between localised states becomes prominent.

\section{Fluctuation induced tunnelling (FIT)}

The fluctuation induced tunnelling (FIT) model was derived by Sheng to describe conduction in long metallic islands separated by thin, insulating barriers [2]. The crux of Sheng's theory is that voltage fluctuations due to thermal energy alter the field between any two metallic islands leading to narrower and lower barriers. These fluctuations have the effect of enhancing tunnelling probabilities through the barrier in the intermediate to low temperature range.

A closed form of the FIT model for a parabolic barrier is given as: 


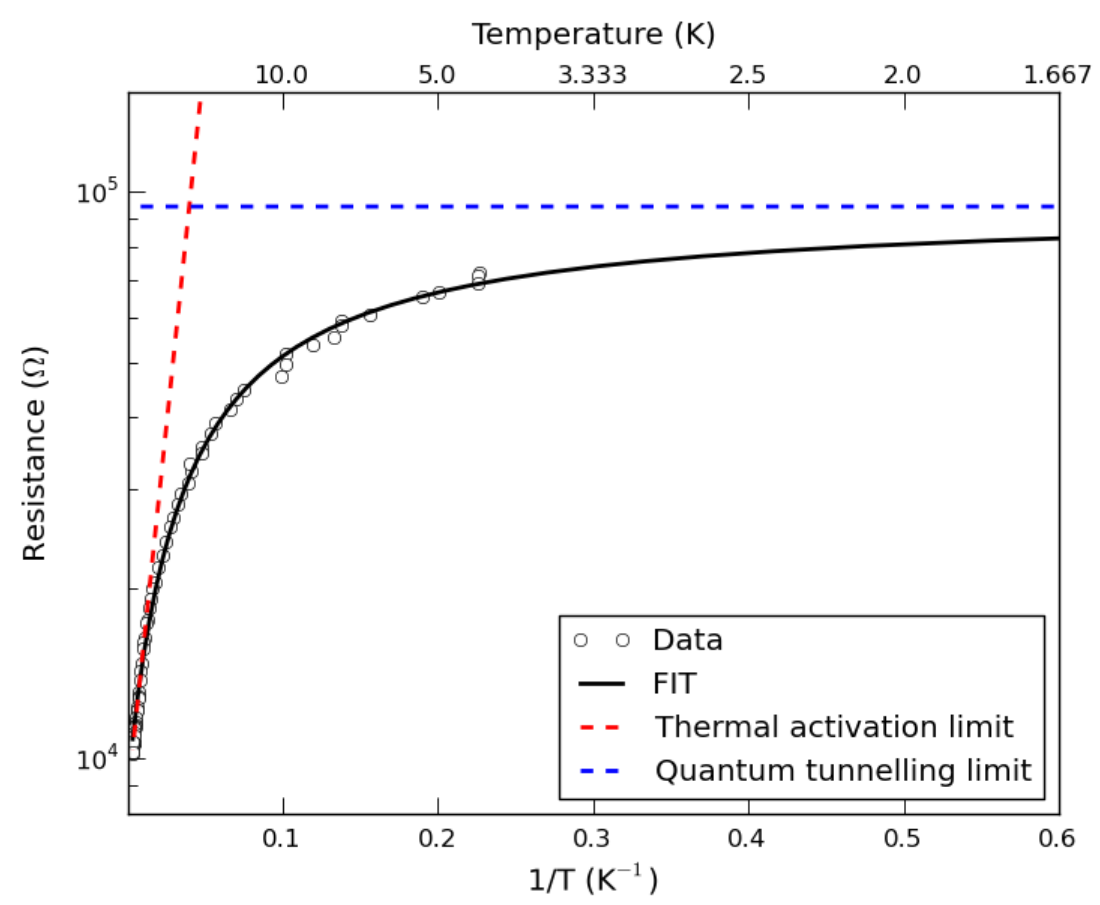

Figure 2.13: Thermal activation and quantum tunnelling limits for metal nanowire/metal contact barriers showing FIT temperature dependence. Data from [30].

$$
G=G_{0} \exp \frac{-T_{B}}{T_{S}+T}
$$

where $T_{B}$ is the amount of energy required for the electron to traverse the potential barrier. This energy is effectively the activation energy. The $T_{S}$ parameter describes the temperature above which thermal fluctuation effects become significant. Or conversely, it can be seen as the temperature below which the temperature dependence given in Eq. 2.24 reduces to the expression for quantum tunnelling i.e. a constant. In the high temperature limit, when $T>>T_{S}$, Eq. 2.24 becomes proportional to $\exp \left(-T_{B} / T\right)$. Thermal activation completely takes over the high temperature regime. This leaves the temperature region $T_{S}$ and $T_{B}$ for fluctuation induced tunnelling alone.

The limits of FIT can be seen clearly in Figure 2.13. Here, the data describe the temperature dependence of conductance between a metal nanowire and the metal contacts - two extended metallic regions separated by an interfacial layer that constitutes a dielectric potential barrier [30]. We can see that as $T \rightarrow 0 \mathrm{~K}$, the conductance tends to a constant value (dashed blue line) due to quantum mechanical tunnelling taking over in the low temperature limit. At high temperatures, where the thermal energy exceeds the barrier energy, thermal activation dominates conduction (dashed red line). At intermediate temperatures, thermal fluctuations enhance tunnelling probabilities through barriers (thick black line). 
The FIT model was initially derived for a single barrier described by parameters $T_{B}$ and $T_{S}$. Sheng later showed that the single barrier model can be generalised to systems resembling a random resistor network, where the resistors represent the tunnelling junctions. The generalisation states that FIT conduction within a system containing a distribution of barriers can also be described by a single barrier with $T_{B}^{m}$ and $T_{S}^{m}$ where $T_{B}^{m}$ and $T_{S}^{m}$ are the median values of the log-normal distribution of $T_{B}$ and $T_{S}$ values for that particular system.

\section{Variable range hopping ( $\mathrm{VRH})$}

The FIT model can be used to describe conduction in systems containing large metallic islands separated by a thin insulating barrier. However, as barriers become large, tunnelling probabilities to and from states on either side of the barrier dwindle to zero. Instead, electrons can tunnel via localised states by absorbing (or emitting) thermal energy. Localised states are numerous in materials with a high degree of static disorder i.e. defects, lattice vacancies, impurities etc. At these sites, the electron wavefunction is localised about a centre and decays exponentially as $\Phi(r) \sim \exp (-r / \xi)$ in space from that point. The parameter, $\xi$, is the localisation length. As in the case with extended wavefunctions, tunnelling through a barrier is possible if the two wavefunctions overlap.

The extended wavefunctions seen in crystals are no longer present in disordered materials like random networks due to a lack of lattice periodicity. Furthermore, these materials also contain a high density of defects. According to Anderson's criteria for localisation, localised states are present within the bandgap and are thus accessible as ports for facilitating conduction. Tunnelling probabilities between localised states are highly dependent on the Fermi window $\left(k_{B} T\right)$. Due to the energy difference between the initial and final states, the electron needs to absorb/emit phonons. Mott's premise for VRH was that electrons can also tunnel to sites further away when the available thermal energy is too low to tunnel to nearby but higher energy sites [3, 31]. This trade-off results in the formula:

$$
G=G_{0} \exp \frac{-T_{0}}{T}
$$

where $d$ is the dimensionality of the available distribution of localised states. The additional dimensionality refers to the dimension of energy that is included in the derivation of the VRH model. The $T_{0}$ parameter is inversely proportional to the localisation length and is defined as 32: 


$$
T_{0}=\frac{16}{n\left(E_{F}\right) k_{B} \xi^{3}}
$$

Eqs. 2.25 and 2.26 assume a constant density of localised electronic states, $n\left(E_{F}\right)$, about the Fermi level. The VRH model of conduction has been shown to apply in many classes of materials. A typical VRH graph, one that exemplifies the characteristic linear dependence of the logarithmic resistance (or conductance) with $T^{-1 / d+1}$, is shown in Figure 2.14. Here, the temperature dependent resistance is plotted on a log scale against $T^{-1 / 4}$ from $60 \mathrm{~K}$ to $300 \mathrm{~K}$ for amorphous silicon thin films of different thickness. The $T_{0}$ parameter can be extracted from the slope of the line and can be used to calculate $\xi$ using Eq. 2.26 for the case of 3D VRH. This localisation length can then be used to infer the effects of various chemical (e.g. doping) or physical treatments to the material.

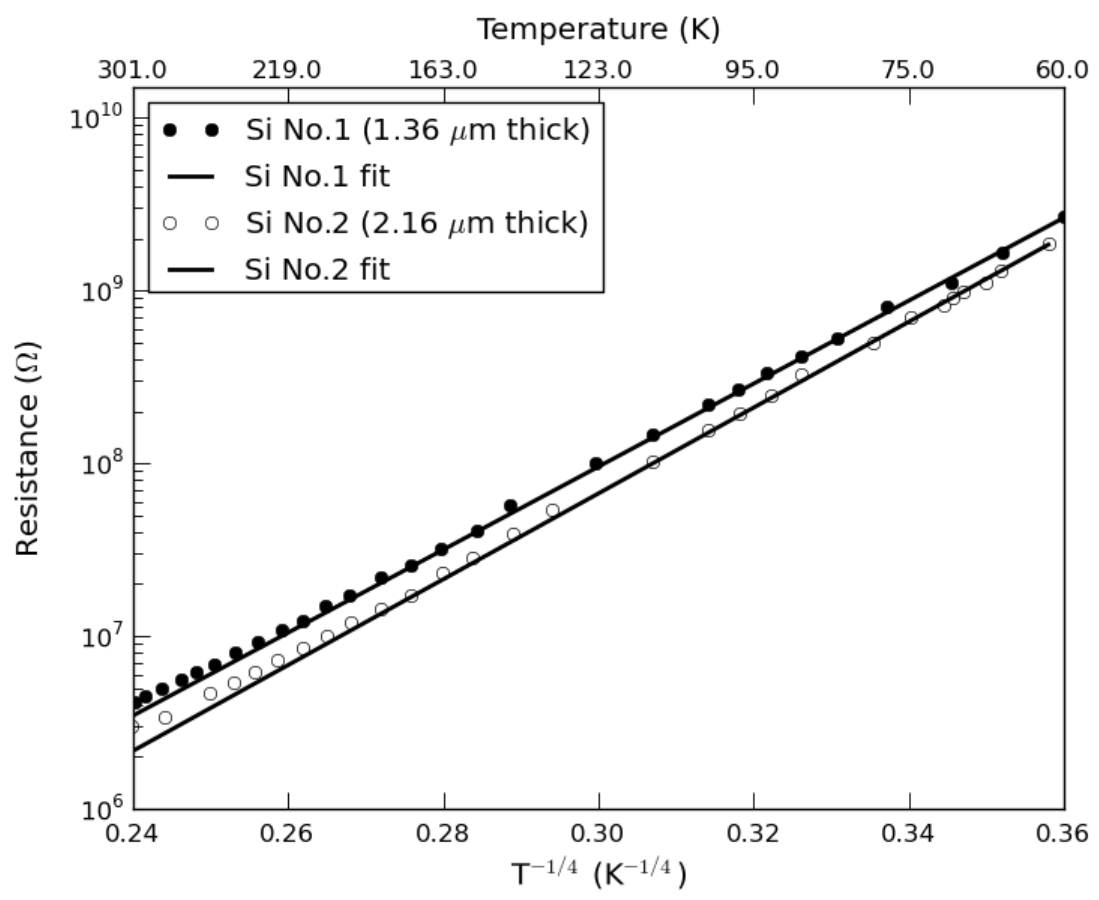

Figure 2.14: Variable range hopping fits to conductivity data of amorphous silicon thin films. Data from [33].

\subsubsection{Summary of conduction mechanisms in SWNT networks}

Figure 2.15 shows how the shape of the temperature-dependence of conductance can be used to identify the type of junction barrier from the mode of transport over/through the junction. Three scenarios are considered in this figure: (A) thermal activation, (B) fluctuation induced tunnelling, and $(\mathrm{C})$ variable range hopping. 


\section{A thermal activation}
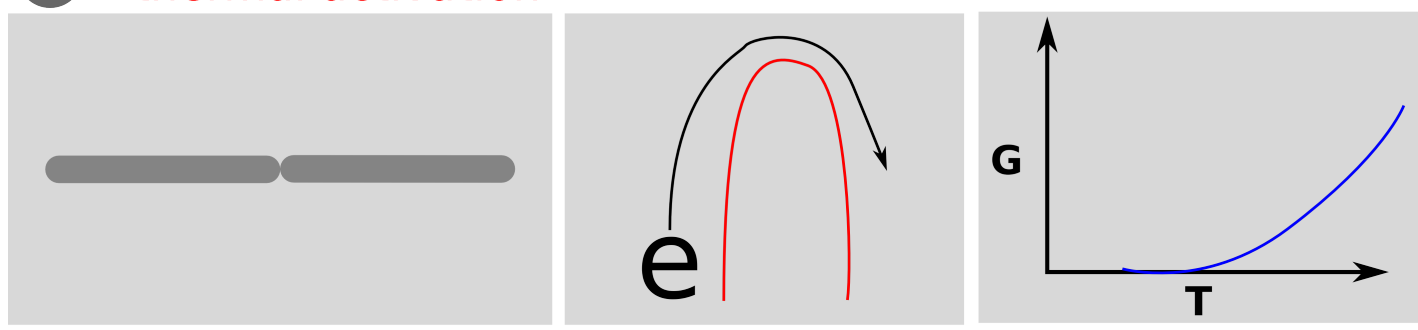

\section{B fluctuation induced tunnelling (FIT)}
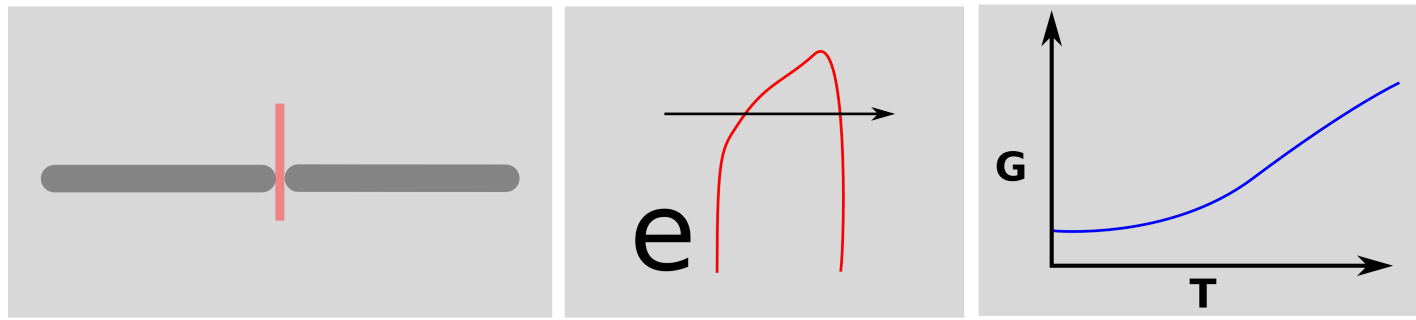

\section{C variable range hopping (VRH)}
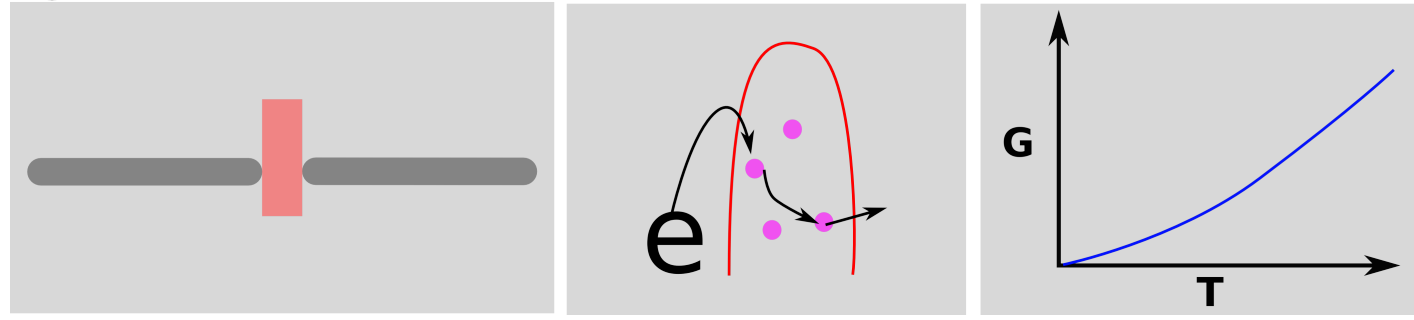

Figure 2.15

Thermal activation (A) typically dominates when the junction barrier/interface only has an energetic component. For example, a clean interface between metallic and semiconducting nanotubes only has an energetic barrier due to mismatched Fermi levels. The remaining two conduction mechanisms of FIT (B) and VRH (C) appear when the junction barrier has a spatial component.

The FIT mechanism is prevalent when (i) the spatial barrier is thin, and (ii) the system has confined dimensions. Carriers can tunnel through the thin barrier if the two nanotubes have a non-zero carrier density at the fermi level. In confined systems, charge carriers can build up on either side of the junction due to thermal fluctuations. The screening effect due to this build-up 'reduces' the barrier size for the carriers and results in an enhanced tunnelling probability. The temperature above which fluctuations affect the tunnelling probabilities is called the 'Sheng temperature' ( $T_{S}$ in Eq. 2.24).

The conductance curve, when the FIT mechanism is prevalent, is dominated by three regions: (i) tunnelling, (ii) fluctuation, and (ii) thermal activation. As discussed in Section 2.3.2. the tunnelling and thermal activation dominate the low and high temperature regions respec- 


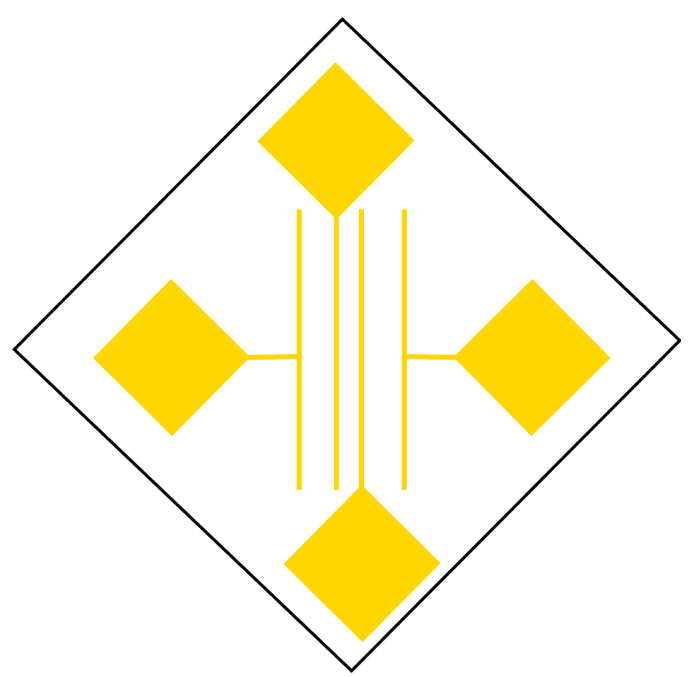

(a) Mask design

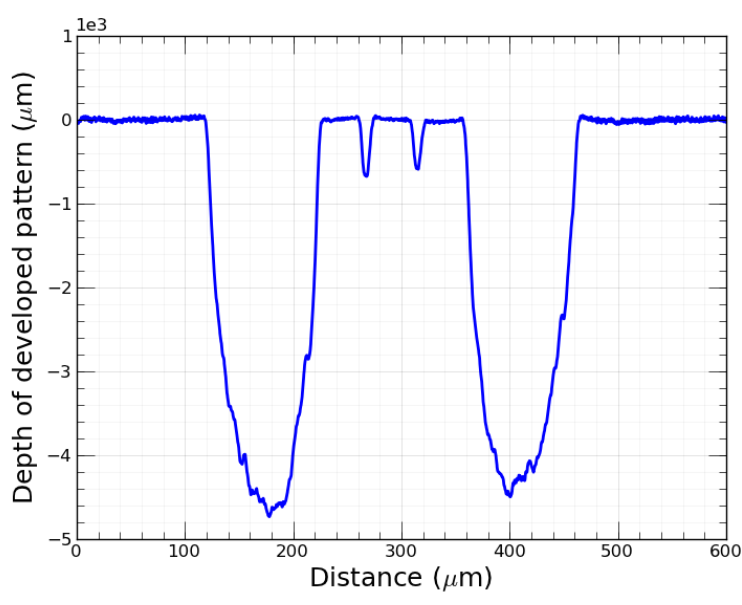

(b) Electrode gaps defined using the Dektak

Figure 2.16: Contact geometry. The length of the electrodes is $2000 \mu \mathrm{m}$. One side of the square contact pads measures $\sim 500 \mu \mathrm{m}$.

tively. The flattened conductance trace, as $T \rightarrow 0 \mathrm{~K}$, represents the tunnelling component. Fluctuations only play a significant role at intermediate temperatures.

The final mechanism, VRH, dominates when the spatial barrier is too large for FIT. Thus, tunnelling probabilities through the barrier (with and without charge fluctuations) are negligible. Carriers can now only traverse the junction barrier by 'hopping' (tunnelling with the aid of thermal energy; see Section 2.3.2 between localised states. The conductance trace looks qualitatively similar to the FIT mechanism with the main exception being that the conductance goes to zero as $T \rightarrow 0 \mathrm{~K}$. Fitting the conductance data to either one of FIT, thermal activation or VRH models will be discussed in detail in the following chapter.

We have discussed conduction mechanisms over junction barriers with different spatial widths. But what are these spatial barriers comprised of? In SWNT networks, they could be anything from residual surfactant, non-conducting nanotubes or, carbonaceous impurities.

\subsection{Conduction: experimental aspects}

\subsubsection{Contacts}

Electrode structures for charge transport studies in $\mathrm{CuO}$ and SWNT networks were fabricated using photolithography in the cleanroom facility at VUW. The mask design that we have used is shown in Figure 2.16. The process of photolithography is summarised in the following procedure.

1. Spin-coat resist at $2000 \mathrm{rpm}$ (accelerating to final speed in 5s) for 1 minute OR, spin-coat 
at $4000 \mathrm{rpm}$ (accelerating to final speed in 15s) for 1 minute.

2. Pre-bake the coated substrate at $95{ }^{\circ} \mathrm{C}$ for 1 minute (or, 1 minute $2 \mathrm{~s}$ ).

3. Expose the pattern for 30s in the mask aligner.

4. Bake the substrate on the hotplate at $110{ }^{\circ} \mathrm{C}$ for 2 minutes.

5. Remove the mask and expose again for another 30s.

6. Develop the pattern in a neat solution of AZ 326 developer for 8-10 s. Full immersion in the developer while moving the sample in the liquid for $8 \mathrm{~s}$ was sufficient if the developer was newly decanted. Longer times were necessary with older, used batches of developer.

The additional steps (steps 4-5) reversed the image since we used the positive lithography AZ5124 resist with a negative mask. After the pattern was developed, chromium and gold metal was evaporated in a thermal evaporator. We typically evaporated 5 - $15 \mathrm{~nm}$ of chromium and around 20 - $40 \mathrm{~nm}$ of gold. The metal coated samples were then soaked in acetone to lift-off the regions with resist. This process typically required over-night soaking in acetone. Unfortunately, finer electrode structures (inner electrodes in Figure 2.16a) which allowed for 4 terminal measurements, were usually not resolved. Finally, gold wire contacts from the square contact pads were made with gold wire using a Kulicke and Suffe 4124 ball bonder.

\subsubsection{Measuring temperature dependent conduction}

All temperature-dependent resistance measurements on SWNT networks were carried out using the Pocket Physical Properties Measurement System (PPMS) at Industrial Research Limited. Samples were loaded onto a resistivity puck (Figure 2.17) three at a time (one sample per channel). Samples were held in place with a thin coating of GE varnish which was cured in an open air furnace (at $100{ }^{\circ} \mathrm{C}$ ) for $\sim 10$ minutes. In some cases when the ball-bonded wire contacts broke off, silver epoxy (EPOTEK H20E) was used to connect to the broken contact. The epoxy was cured according to instructions: for either 30 minutes at $50{ }^{\circ} \mathrm{C}$ or 10 minutes at $100{ }^{\circ} \mathrm{C}$. Data was collected at a constant current of $0.005 \mu \mathrm{A}$ in the 'standard' collection mode. Connections to the outer contact pads were made with solder.

The PPMS automates most of the physical processes with in-built software, for example, the evacuation and purging process of the sample chamber with the 'Purge' option in the software. The sample chamber is evacuated with a roughing pump and purged with helium gas several times before the pressure settles to a few Torr of helium. Similarly, the venting process to ambient is activated with the 'Vent/Seal' option and the pump/gas systems cycle through a set of prescribed steps. Data logging is also automated with built-in electronics that can only be controlled through a menu in the software. Resistance data was acquired between 400 and 


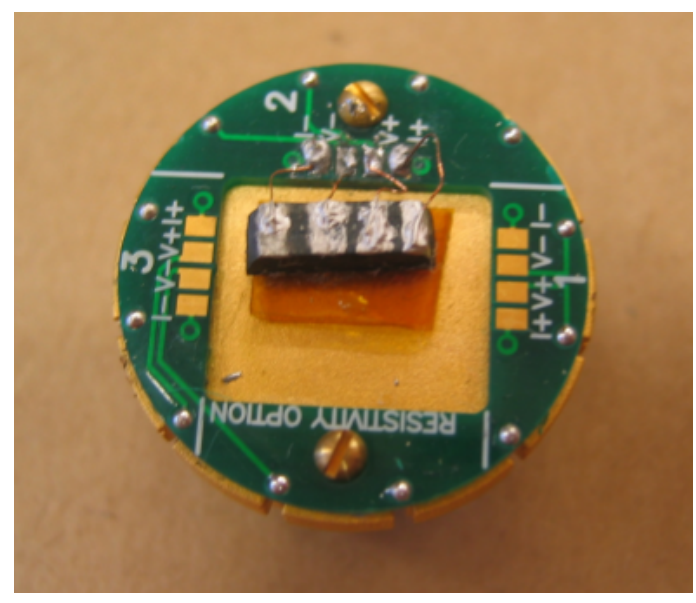

Figure 2.17: Resistivity puck for PPMS. The three channels are labelled in white.

$10 \mathrm{~K}$ every $0.5 \mathrm{~K}$.

A small study on charge transport in cupric oxide thin films was carried out using a Janis cryostat at VUW. Sample resistance was measured between 100 and 300 K. A Keithley source measure unit was used to collect I-V (current-voltage) curves at defined points within the measured temperature range. I-V curves were measured between $\pm 10 \mathrm{~V}$ in $1 \mathrm{~V}$ steps.

\subsection{X-ray diffraction}

X-ray diffraction (XRD) was used to study grain sizes and intrinsic stress in our cupric oxide samples. X-ray diffractograms were collected using the PANalytical X'Pert Pro diffractometer, in the Bragg-Brentano geometry, with a copper x-ray source. It is essential that the XRD diffractometer is well calibrated and characterised for accurate crystallite dimensions extracted from the measured peak shifts and linewidths. The following section describes the procedure we followed to identify the instrumental calibration for XRD linewidths. We additionally identify the calibration for the separation between the $k \alpha_{1}$ and $k \alpha_{2}$ peaks. The copper source has three prominent lines: $k \alpha_{1}, k \alpha_{2}$ and $k \beta$. The $k \beta$ line can be filtered with a special beta filter. However, the two closely-spaced $k \alpha$ lines remain. Thus, every reflection is in fact a doublet in the XRD pattern. The calibration curve of the $k \alpha$ line separation allows broad diffraction peaks from nanostructured materials to be deconvoluted with accuracy (Chapter 7.4.1).

A silicon standard (from PANalytical) was used to calibrate the instrumental broadening and the $k \alpha_{1}$ and $k \alpha_{2}$ peak separation. Diffractogram analysis was performed by dividing the silicon diffractogram into $\mathrm{N}$ observable peak regions (in this case $\mathrm{N}=7$ ). A linear background was fitted to each region and subtracted from the signal. An analytical function that took into account the doublet nature of each peak was used for fitting. Initially, a symmetric line profile - Pearson VII - was used to fit each silicon peak (see Eq. 2.27) where the parameters $a, x_{1}, x_{2}, b$ 
and $m$ refer respectively to the intensity of the $k \alpha_{1}$ peak, the position of the $k \alpha_{1}$ peak, position of the $k \alpha_{2}$ peak, a factor proportional to the linewidth and the peak shape factor. The intensity of the $k \alpha_{2}$ peak is half that of the main $k \alpha_{1}$ peak (Table 1-3 [34]) thus, its intensity is constrained as $a / 2$ in Eq. 2.27. The peak shape factor, $m$, describes the peak shape, with $m=1$ being a Lorentzian, and $m=\infty$ a Gaussian. Both the peak shape factor and the parameter, $b$, are used to evaluate the linewidth: $\Gamma$ or, full-width at half-maximum - FWHM, (Eq. 2.28).

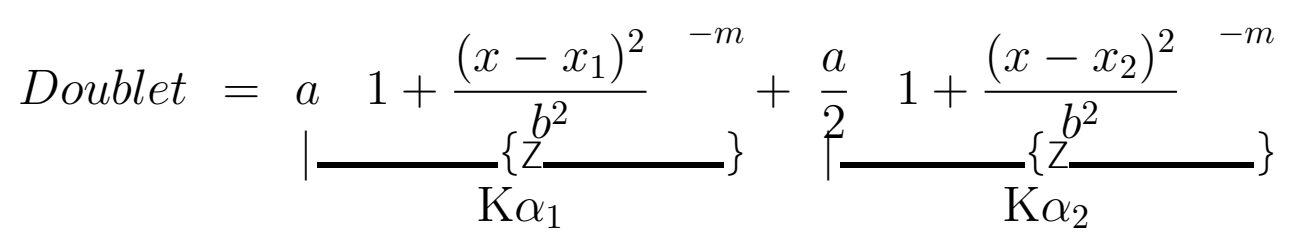

$$
\Gamma=2 b \quad 2^{\frac{1}{m}}-1^{0.5}
$$

However, we found that a symmetrical lineshape failed to adequately represent the instrumental broadening of our instrument for reflections below $2 \theta \sim 70^{\circ}$. As can be seen from Figure 2.18a, the $k \alpha_{1}$ and $k \alpha_{2}$ peaks obtained from our crystalline silicon standard are visibly asymmetric in the low angle regions. To accommodate this asymmetry, the peaks were fit using a split-Pearson VII function (see Eq 2.29 and Figure 2.19ab).

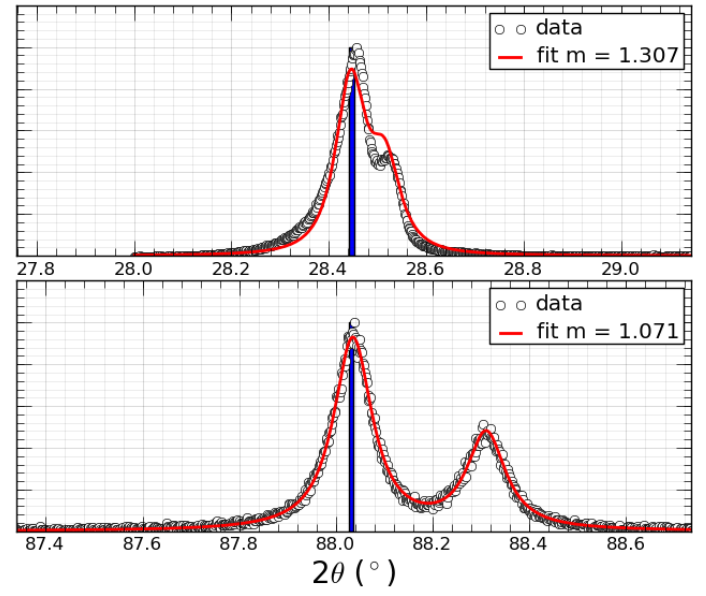

(a) Pearson VII lineshape (symmetric)

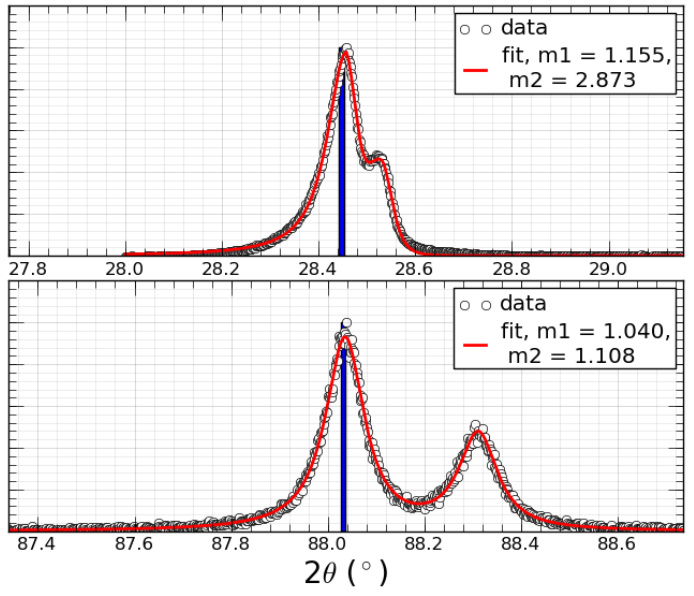

(b) Split-Pearson VII lineshape (asymmetric)

Figure 2.18: Silicon Calibration: choice of symmetric and asymmetric lineshapes. The blue bar indicates the position of the peak according to the silicon reference. 


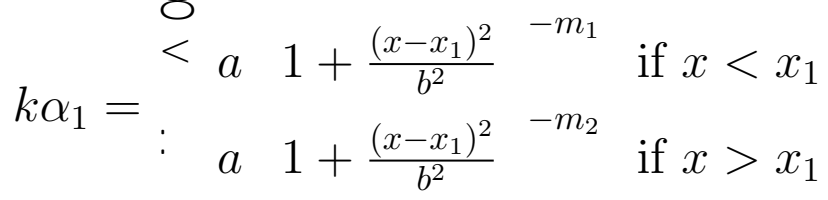

$$
\begin{aligned}
& k \alpha_{2}=\begin{array}{lll}
0.5 a & 1+\frac{\left(x-x_{1}-\delta\right)^{2}}{b^{2}} & -m_{1} \\
0.5 a & \text { if } x<x_{1}-\delta \\
b^{2} & \text { if } x>x_{1}-\delta
\end{array}
\end{aligned}
$$

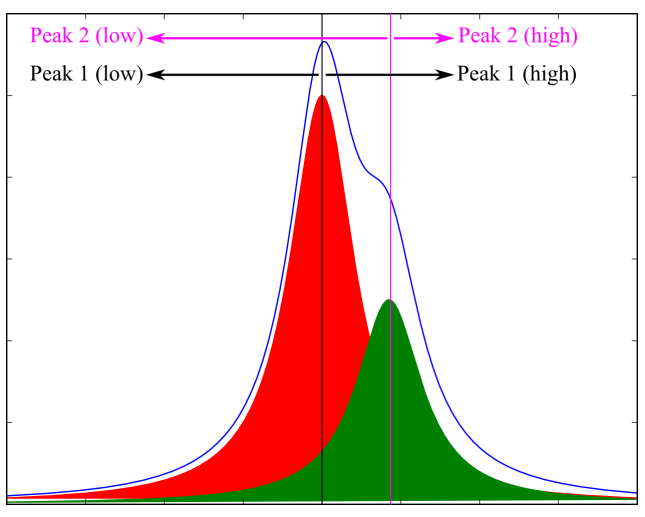

(a) Splitting the doublet

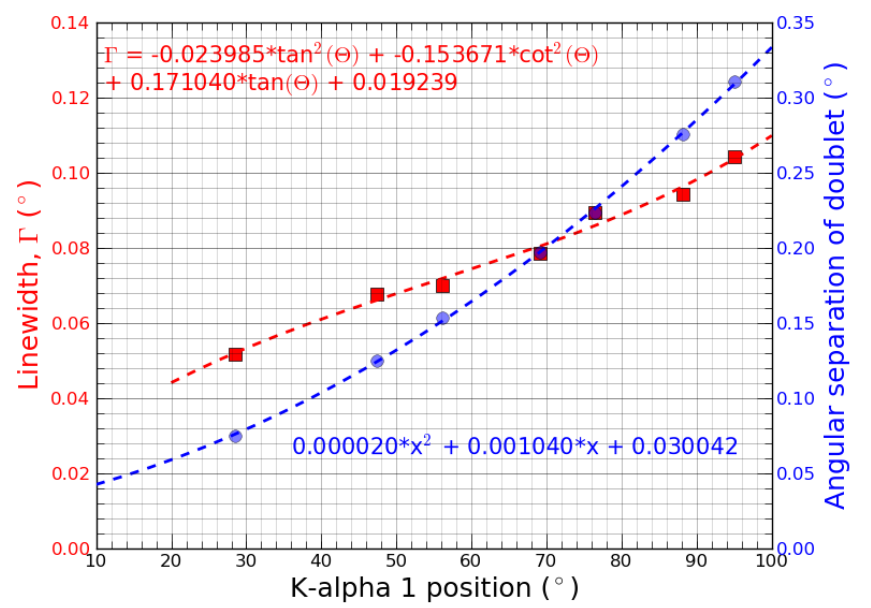

(b) Instrumental broadening and $\mathrm{K} \alpha$ doublet separation

Figure 2.19: Procedure for fitting the silicon diffractogram with the split-Pearson VII function and results for fitting analytical forms of instrumental broadening and $k \alpha$ doublet separation.

Fitting a linear combination of Eq. 2.29 and Eq. 2.30- where $\delta$ is the $k \alpha$ doublet separation - to the silicon diffractogram now better describes the observed peak profiles (Figure 2.18b). The parameters $m 1, m 2, b, x_{1}$ and $x_{1}$ were extracted from these fits and used to evaluate the $k \alpha$ doublet separation and linewidth as a function of $2 \theta$. The doublet separation data was fitted with a third-order polynomial to give a continuous analytical function (Figure 2.19b), enabling calibration across the entire range of the instrument. An analytical description of the instrumental linewidth is complicated by the asymmetric nature of the instrumental peaks. We have chosen to define the instrumental broadening as that occurring in the high angle tail of the peak, since the low angle tail of the asymmetric peaks is a result of broadening due to axial divergence of the incident beam optics. An analytical function for fitting the diffractometer linewidth follows the form shown in Eq. 2.31 [35]. The fitted values for silicon data using Eq. 2.31 (see the red curve in Figure 2.19b) are used to correct data for instrumental broadening. 


$$
F W H M=A \tan ^{2}(\theta)+B \cot ^{2}(\theta)+C \tan (\theta)+D
$$

\subsection{Other experimental techniques}

Several of the fabrication steps within this study required annealing at high temperature. Figure 2.20 shows the quartz tube furnace where high temperature annealing was carried out. Annealing temperatures were set using an attached temperature controller. For the fabrication of $\mathrm{CuO}$ nanowire forests, an aquarium pump was to connected to one of the tube inlets via a water bubbler.

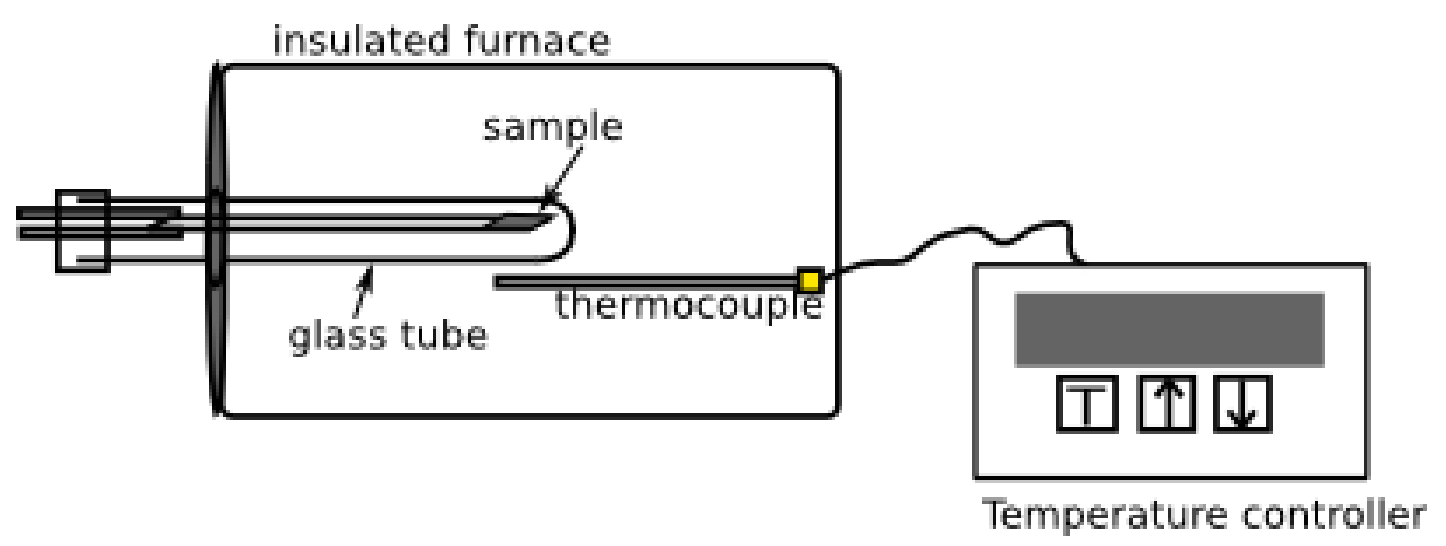

Figure 2.20: Tube furnace used for high temperature annealing of SWNT networks and for fabricating $\mathrm{CuO}$ thin films and nanowire forests.

Scanning electron microscopy (SEM) was done using a JEOL JSM 6500F microscope at VUW. Since SEM was only used for imaging, we do not go into the details for this characterisation technique.

Thermogravimetric analysis (TGA) and differential scanning calorimetry (DSC) were carried out simultaneously for a wet pellet of SWNTs. These were prepared in a TGA pan, heated to $45 \circ \mathrm{C}$ to remove the bulk of the liquid solvent, from a dispersion of SWNTs in neat nbutylamine. The runs were performed to $600 \mathrm{C}$ at a rate of $10{ }^{\circ} \mathrm{C} /$ minute under a $50 \mathrm{~mL} / \mathrm{min}$ gas purge of dry air and, nitrogen. A control thermogram of a dry pellet was obtained at the same ramping conditions in dry air. 


\section{Chapter 3}

\section{Introduction to single-walled carbon nanotube networks}

\subsection{Introduction}

Devices like solar cells, electronic displays and light emitting diodes require electrodes to both regulate operations and allow light transmission into (for solar cells) or out of (displays and LEDs) of the device. The study of transparent conducting materials has featured prominently over the last few decades given the rising market demand for solar cells and LCDs. The class of materials, known as 'transparent conducting oxides' or TCOs, are the most well known and widely used transparent conductors. They are fabricated using a heavily doped semiconductor where the shallow dopants give rise to a degenerate electron gas within the material without affecting the optical bandgap of the host lattice. Indium tin oxide (ITO) is the current industry standard.

ITO is indium oxide doped with tin oxide. The host, indium oxide, has a bandgap of $\sim$ $3.75 \mathrm{eV}$. At heavy doping concentration (free carriers $>10^{19} \mathrm{~cm}^{3}$ ), the material undergoes a Mott transition to the metallic state [36]. In this metallic phase, ITO can attain conductivities around $10^{6} \mathrm{~S} / \mathrm{m}$ for optical transparency greater than $80 \%$ [36]. However, the transparency for ITO only extends to the near infra-red before Drude absorption by the free carriers reduces the transparency of the material. The morphology of ITO also prevents its use in flexible electronic devices - an avenue that is being actively explored by the display industry. ITO is typically fabricated by a sputtering/evaporation process and comprises of nano to micro-sized islands. Stress and strain to the substrate breaks the connections between the islands and reduces the performance of the thin film. The search and study of replacement materials that address these performance shortcomings of ITO drives much of the current research. The main criteria for new materials are sheet resistance impervious to mechanical stress and a sheet resistance below $100 \Omega / \square$ (ideally $10 \Omega / \square$ for current driven applications) at $85 \%$ transparency. Another significant criterion is that of reducing cost. The price of ITO is limited by a complex fabrication process and the generally increasing trend in the price of indium [37]. 
Several types of transparent conductors are being studied as possible ITO replacements. One strategy to reduce opacity, while maintaining high conductivity, is by moving to lower surface coverage structures like grids. Regular metallic grids, with dimensions less than the wavelength of visible light, have been recently demonstrated using lithography [38, 39]. Such nanoscale grids can attain low sheet resistances of $\sim 24 \Omega / \square$ at $85 \%$ transparency [38]. However, fabrication by lithography is a complex and expensive technique not to mention time consuming. A workaround is to create a less structured material (one that doesn't need to be fabricated by lithography) from the basic component and underlying principle of a grid array. One line of a nanoscale grid is a single conducting path with a micron in length and sub-micron in diameter. This basic component can form a randomly oriented network resembling a game of pick up sticks. At some critical coverage, the percolation threshold, long range connectivity appears and current carrying pathways are formed. With the addition of more nanowires, lower resistance pathways and parallel conduction paths take over the load and lower the sheet resistance of the network. Such a system of parallel conducting paths is analogous to that of a grid which has multiple conducting paths formed by each row and column. Lee et al. found that the optoelectronic properties of randomly oriented metallic nanowires (silver, in this case), rivalled those of the silver grids fabricated by lithography [39]. Further details on the dependence of percolation on nanotube diameter and length can be found two well-cited works [40, 41].

The advantage of random meshes over patterned grids lies in the ease of fabrication. Networks can be deposited by applying an ink (containing the nanowires dispersed in some solvent) to a surface and allowing the solvent to evaporate. Until recently, the difficulty of fabricating nanowires limited their utilization in macroelectronics. This limitation has been overcome by advances in solution processing and other techniques that produce high quality nanowires at high yields [42].

SWNT networks as macroelectronic devices hold several advantages over nanowire networks: they can be synthesised from simple precursors like carbon monoxide or methane and can be either semiconducting or metallic. A single material for both the semiconducting and the metallic componentry in a device could go a long way towards considerable reductions in production costs. In this thesis, we are primarily focused on SWNT networks with metallic characteristics. However, the diagnostic tools (discussed later) will be applicable to any field utilising SWNT networks; both as semiconducting/metallic components.

Carbon nanotube networks prepared with an optimised conductivity of $6 \times 10^{5} \mathrm{~S} / \mathrm{m}$ compare favourably with that of ITO. They can be prepared directly on, or transferred to plastic from solution. The entangled morphology gives the material considerable fault tolerance. Electromechanical studies of nanotube networks on plastic (PET) have reported a relatively poor dependence of electronic performance on mechanical stress [43]. Though the cost of a gram 
of nanotubes (USD400/g) [4] is orders of magnitude from the price of indium (USD0.6/g), milligram quantities are sufficient for a litre of nanotube solution. Ball-park figures of the production costs of carbon nanotube and ITO films have been calculated to be $\$ 64 / \mathrm{m}^{2}$ [45] and $\$ 100 / \mathrm{m}^{2}$ (for good quality ITO).

Recent research into carbon nanotube networks has been primarily focused on improving optoelectronic characteristics to the industry maximum of $10 \Omega /$ sq at $85 \%$ transparency. The optical conductivity $\left(\sigma_{A C}\right)$ depends only on the density of material while the DC electrical conductivity $\left(\sigma_{D C}\right)$ depends on quality of conducting pathways within the network [46, 47]. The DC conductivity is the limiting factor since optical conductivities are typically around 2 $\times 10^{4} \mathrm{~S} / \mathrm{m}$ [29, 45] for carbon nanotube networks. The quality of DC conductivity for a given coverage of SWNTs (optical conductivity) can be obtained from the ratio of $\sigma_{D C} / \sigma_{A C}$. The best ratio achieved so far $\left(\sigma_{D C} / \sigma_{A C}=35\right)$ just meets the minimum industry requirement of $100 \Omega /$ sq and $>90 \%$ [29]. Considerable improvements to the DC conductivity are required to attain the industry maximum $\left(\sigma_{D C} / \sigma_{A C}=330\right)$.

While ballistic transport dominates within SWNTs [48], a network constructed of these nanotubes is limited by inter-nanotube junctions [28]. The junctions have an associated 'barrier' energy and the way charge carriers overcome these barriers dictates the quality of the conducting pathways. Typically, low resistance networks are described by interrupted metallic conduction (FIT) or, metallic pathways separated by small barriers. Higher resistance networks contain larger barriers that can only be overcome by the weaker process of phonon-assisted hopping (VRH). These conducting segments between these larger barriers can be metallic or non-metallic. The theoretical aspects of these two conduction mechanisms are covered in Chapter 4.4.

Barrier size (or distribution of barrier size) is not a constant and can be tuned by physical or chemical means. Better conducting paths can be engineered by physically altering: (i) the metallic:semiconducting (M:S) ratio of the nanotube sample [49, 50, 51] or, (ii) the surface coverage of nanotubes (tube density/thickness) [47]. Semiconducting tubes in heterogeneous networks can contribute to current-carrying pathways by (i) doping [29] or (ii) by reducing the spatial distance between bundles which can activate metal-semiconductor junctions [52]. The options of surface coverage and doping provide a semi-continuous 'knob' for tuning the barrier size without altering the nanotube starting material. Gradual chemical doping of the network may be achieved by exposure time [53] or by the strength of the chemical dopant [54]. The degree of surface coverage is controlled by adjusting the amount of deposited nanotubes. The extent to which these parameters affect the barrier size can be tested by analysing the temperature dependence of the resistance.

This chapter begins with a brief introduction to the properties of SWNTs. The following 
section describes how doping and tube density affect the barrier size for HiPCO nanotubes (our chosen starting material), in particular. In Section 3.4, we briefly outline our strategy for connecting the macroscopic tuning parameter of tube density to the microscopic picture of junction barriers. Section 3.5 describes a parallel study on characterising the underlying chemical (e.g. extent of ambient $\mathrm{O}_{2}$ doping) and physical (e.g. morphology) makeup of the samples using Raman spectroscopy.

\section{$3.2 \quad$ Geometry and electronic structure}

The unique electronic properties of SWNTs can be constructed from the simple geometric parameters that determine nanotube structure. A conceptual construction of a nanaotube (illustrative only) is described below. Starting from a graphene sheet (Figure 3.1), we see that the sheet itself can be cut along an angle $\theta_{C}$. Due to the six-fold symmetry of the hexagonal lattice and the additional helical degeneracy (right-handed and left-handed helices have the same proprerties), this angle is between $0^{\circ}$ and $30^{\circ}$. The chiral vector $\left(\overrightarrow{C_{h}}\right)$, the radial perimeter of the nanotube, is determined by the linear combination of the two graphene basis vectors, $n \mathbf{a}_{1}+m \mathbf{a}_{2}$, with lengths $n$ and $m$ respectively. These two lengths are typically represented within parentheses: $(n, m)$, and referred to as the chiral index. The values of the index are unique and, as discussed below, underpin the electronic characteristics. Figure 3.1 shows three different nanotubes that can be generated by cutting a rectangle out of the graphene sheet along $\overrightarrow{C_{h}}$ and $\vec{T}$, the translation vector, which can also be defined in terms of $n$ and $m$ [55]. A nanotube is formed by rolling the graphene sheet into a seamless cylinder,along $\vec{C}_{h}$, of length $\vec{T}$ and capping the ends with half a fullerene.

$(4,2)$

$(4,4)$

$(6,0)$

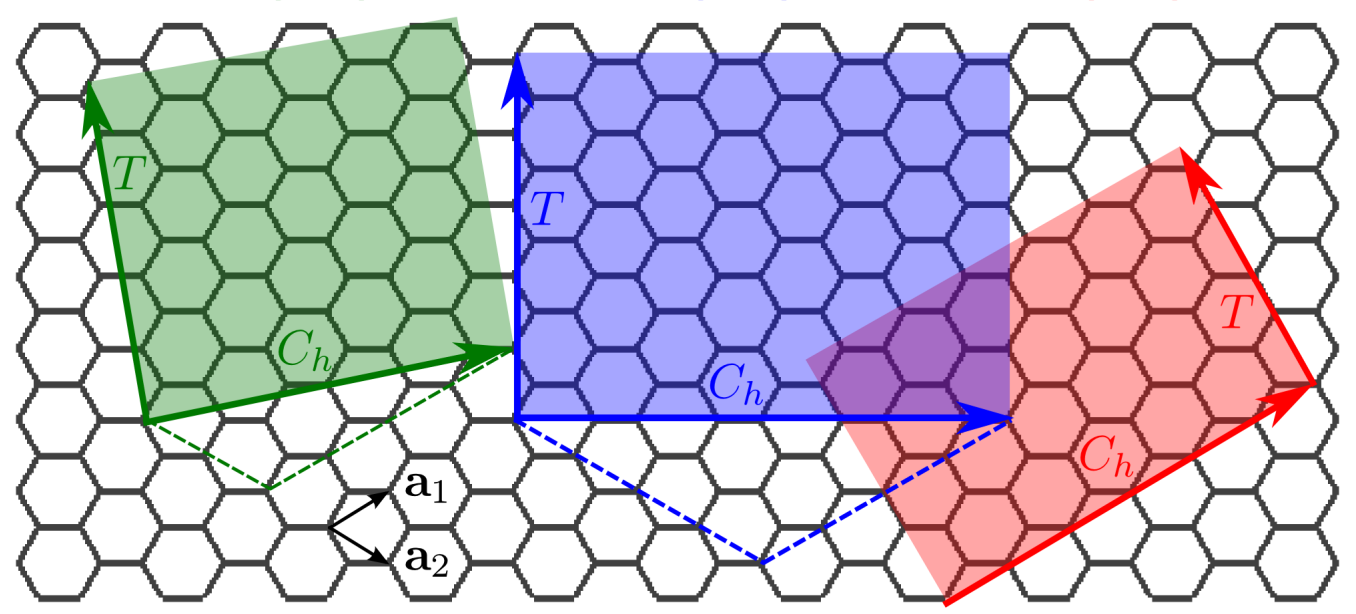

Figure 3.1: Cutting the $(4,2),(4,4)$ and $(6,0)$ nanotubes from a graphene sheet. The coloured dashed lines represent the lengths, $(n, m)$, of the two component basis vectors. 
Since the single-walled carbon nanotube is a quantised form of graphene, its electronic structure can be derived from that of graphene. The bandstructure is constructed with Eq. 3.1. which is derived using the tight-binding approximation [56]. The parameters $\gamma_{0}, s$ and $a$ stand for the carbon-carbon interaction strength or transfer integral (2.9 eV), overlap integral (0.129) and graphene lattice constant $(2.46 \AA)$ respectively. The semi-metallic properties of graphene arises from a few regions (at the $\mathrm{K}$ points) in the bandstructure where the valence and conduction bands touch (i.e. zero bandgap).

The bandstructure for SWNTs (Eq. 3.3 where $i=1,2,3 . . \mathrm{N}$ : the number of unit cells in a given nanotube) is effectively Eq. 3.1 with the additional criterion of quantisation along the circumferential direction, due to the rolling of the graphene sheet. The reciprocal lattice vectors $\overrightarrow{K_{1}}$ and $\vec{K}_{2}$ are defined as $2 \pi / \overrightarrow{C_{h}}$ and $2 \pi / \vec{T}$ respectively. This quantisation of the $\vec{K}_{2}$ reciprocal lattice vector while retaining continuity in the $\vec{K}_{1}$ direction creates discrete 'cutting lines' along the graphene Brillouin zone (Figure 3.2). The nanotube dispersion relation is a series of $\mathrm{N}$ bands (which are the cross-sections of the graphene dispersion relation at $i \vec{K}_{1}$. A metallic tube results when the cutting lines cross the $\mathrm{K}$ point of the graphene Brillouin zone. The geometric conditions for metallicity are given below in Table 3.1. All other tubes are chiral and semiconducting.
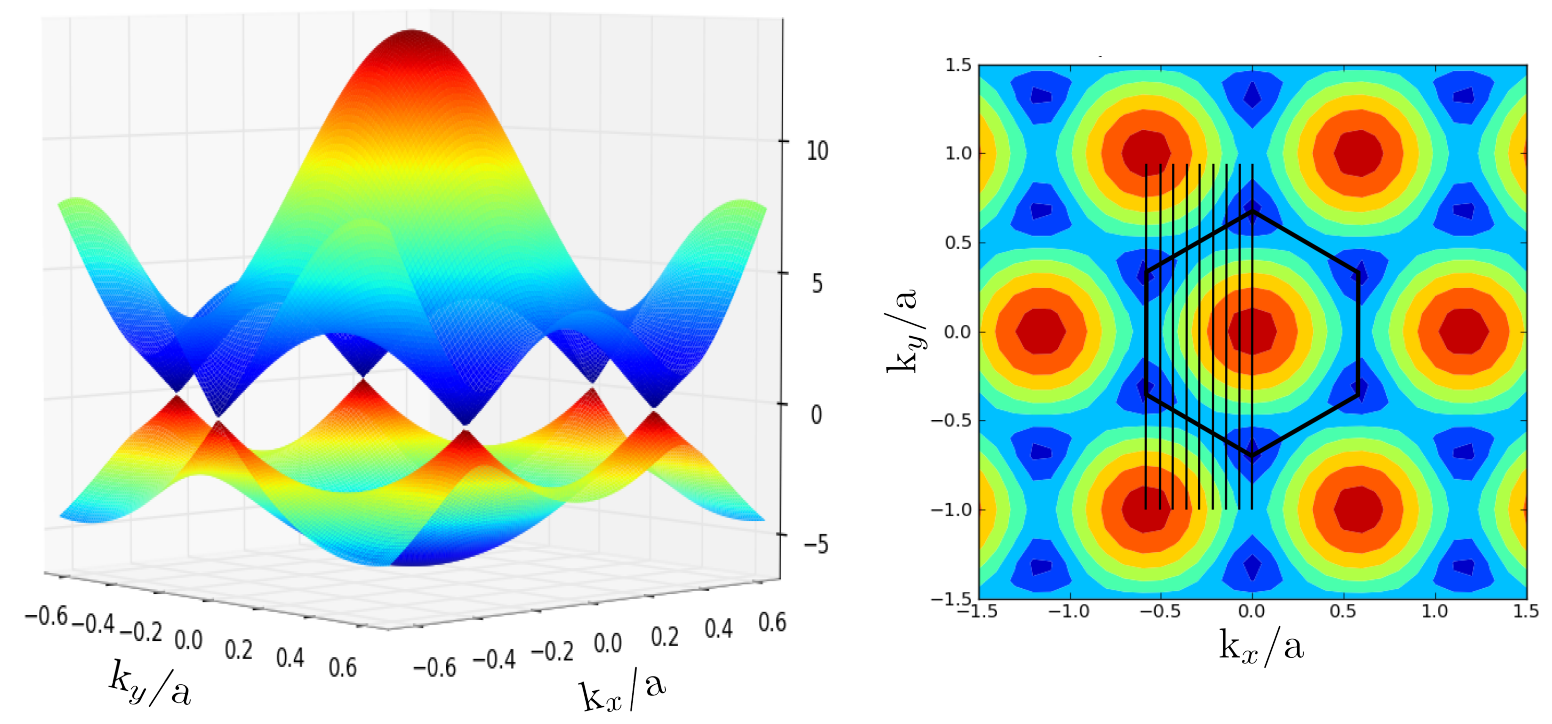

Figure 3.2: Graphene dispersion relation and contour map plotted using Eq. 3.1 and Eq.3.2. The hexagon in the contour map depicts the first Brillouin zone. The cutting lines represent the allowed $k_{y}$ vectors at discrete values of $k_{x}$. In this case, the cutting lines cross the corner $\mathrm{K}$-points which indicate that the nanotube is metallic.

$$
E_{\text {graphene }}=\frac{ \pm \gamma_{0} w(k)}{1 \mp s w(k)}
$$




$$
w(k)=\stackrel{\mathrm{v}}{\mathrm{u}} 1+4 \cos \frac{\sqrt{3} k_{x} a}{2} \quad \cos \frac{k_{y} a}{2}+4 \cos ^{2} \frac{k_{y}}{2}
$$

$$
E_{S W N T}=E_{\text {graphene }} \quad k \frac{K_{2}}{\left|K_{2}\right|}+i K_{1}
$$

\begin{tabular}{|c|c|c|c|c|}
\hline Electronic type & $n$ & $m$ & Condition & Chirality \\
\hline \hline Metallic & $n$ & 0 & & Achiral \\
\hline Metallic & $n$ & $n$ & & Achiral \\
\hline Metallic & $n$ & $m$ & $n-m=3 l$, where $l=$ integer & Chiral \\
\hline
\end{tabular}

Table 3.1: Geometric conditions for SWNT electronic type.

The density of states (DOS) can be calculated from the bandstructure (Eq. 3.3) for any nanotube. Figure 3.3a shows the archetypal DOS for a one-dimensional material containing the discontinuities known as van Hove singularities (VHS). We can see that metallic SWNTs (red and blue lines) contain free carriers at the Fermi level - non-zero DOS - while semiconducting tubes (green line) have a bandgap. Electronic transitions between the valence and conduction VHS are denoted as $E_{i i}$, where $i$ is the 'ith' transition. For example, a transition from the first VHS valence band to the first VHS conduction band is $E_{11}$. Asymmetric transitions, or $E_{i j}$, are considerably weaker than $E_{i i}$ transitions [57]. The first transition, $E_{11}$, is the bandgap for semiconducting tubes. Figure 5.3 shows that this gap is inversely dependent on diameter. This diameter dependence persists even for the higher order transitions.

\subsection{Tuning electronic properties of SWNT networks}

The geometric tunability of SWNTs is promising for the realisation of intricate molecular electronics but current synthetic routes cannot produce nanotubes of one electronic type. Asprepared SWNTs are a heterogenous mixture of metallic (M) and semiconducting (S) tubes. Experimental values for the M:S ratios for some common fabrication processes are given in Table 3.2. The theoretical M:S ratio can be estimated from the union of two independent sets of data: all the possible nanotubes (from $n, m=0$ to $n, m=40$ ) [58], and the diameter distribution for a given type of nanotubes. The diameter distribution for HiPCO SWNTs used in this study can be modelled with a gaussian: mean diameter of $1.05 \mathrm{~nm}$ and standard deviation of $\sigma=0.15 \mathrm{~nm}$ [59]. Thus, the theoretical M:S ratio for HiPCO SWNTs is 0.35:0.65 or 1:2; a ratio that matches the experimentally established ratio (Table 3.2). The similarity between the two values suggests that HiPCO tubes have a uniform chirality distribution. In the theoretical 


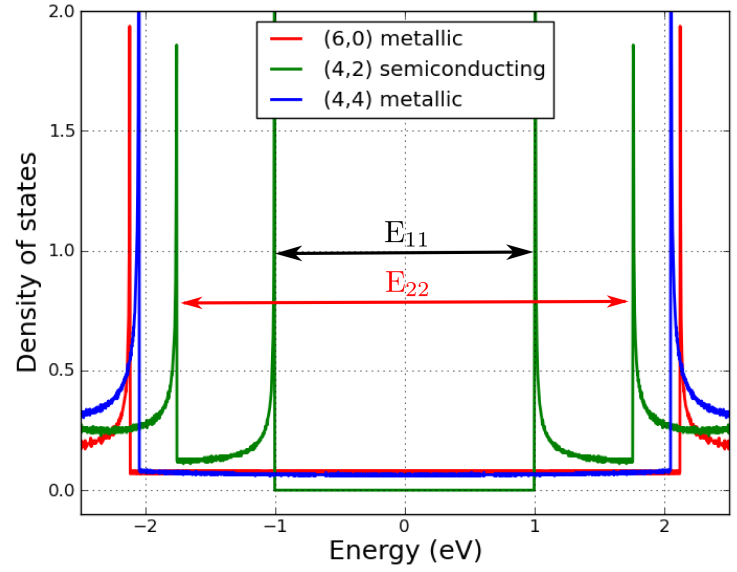

(a) Doping effects

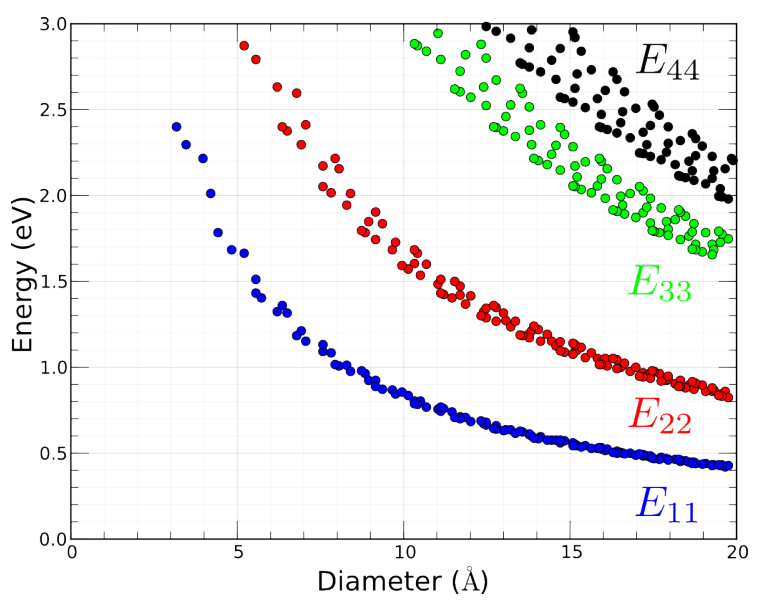

(b) Diameter dependence of $E_{i i}$

Figure 3.3: (a) Density of states for metallic and semiconducting tubes. Data for plot obtained from [58]. The electronic transitions are labelled $E_{i i}$. (b) A Kataura plot from [58] showing how the $E_{i i}$ depend on diameter. This plot only shows the data for the semiconducting tubes but metallic tubes also follow the same behaviour.

\begin{tabular}{|c|c|c|}
\hline Process (Source) & \% Semiconducting & \% Metallic \\
\hline \hline HiPCO (Rice University) & 62.9 & 37.1 \\
\hline CoMoCAT (SWeNT Inc. Standard Grade) & 92.1 & 7.9 \\
\hline CVD (Honda Res. Inst. USA Inc) & 15.4 & 84.6 \\
\hline Arc discharge (Carbon Solutions Inc.) & 67 & 33 \\
\hline Arc discharge (Hanwha) & 10 & 90 \\
\hline
\end{tabular}

Table 3.2: Enrichment by electronic type for SWNTs produced by different processes. These values for HiPCO, CVD and CoMoCAT were obtained experimentally using photoluminescence and AFM [61]. Enrichment ratios for arc-discharge tubes from Carbon Solutions and Hanwha are obtained from [44] and [62] using absorption spectroscopy respectively. From the information provided, it cannot be determined how the ratio from [44] was obtained.

estimate, all the possible combinations had an equal weighting, which, does not always hold true in experiment. Certain processes strongly favour certain types of tubes (by chiral angle, or even $(n, m))$ [60].

While detrimental for applications requiring purity in electronic type, the intrinsic heterogeneity of SWNT samples has one advantage: it allows for the tuning of macroelectronic properties from semiconducting to metallic using a percolation strategy i.e. dependent only upon nanotube density. The tube density can be controlled by altering either the weight concentration in solution or, the deposition volume. The former is employed in most ex-situ percolation studies, where samples are prepared in advance. The sheet resistance follows a power law behaviour when plotted against tube density (SWNT weight concentration) [47]. The exponent qualitatively represents the dimensionality of percolation but is not always comparable to theory. 
Networks near the percolation threshold exhibit semiconducting behaviour [27, 63, 64, since the number of metallic pathways is almost zero; connected pathways are made up mostly of semiconducting tubes. Not far above this threshold, films contain sufficient metallic pathways to short the semiconducting paths. The onset of metallic characteristics varies depending on the fabrication procedure. Figure 3.4 shows the data for one such study where films with a sheet resistance from tens of $\mathrm{M} \Omega$ to tens of $\mathrm{k} \Omega$ exhibit semiconducting character. In other cases, metallic tubes short the connectivity even at very sparse tube densities and must be burned off in order to observe semiconducting behaviour [65].

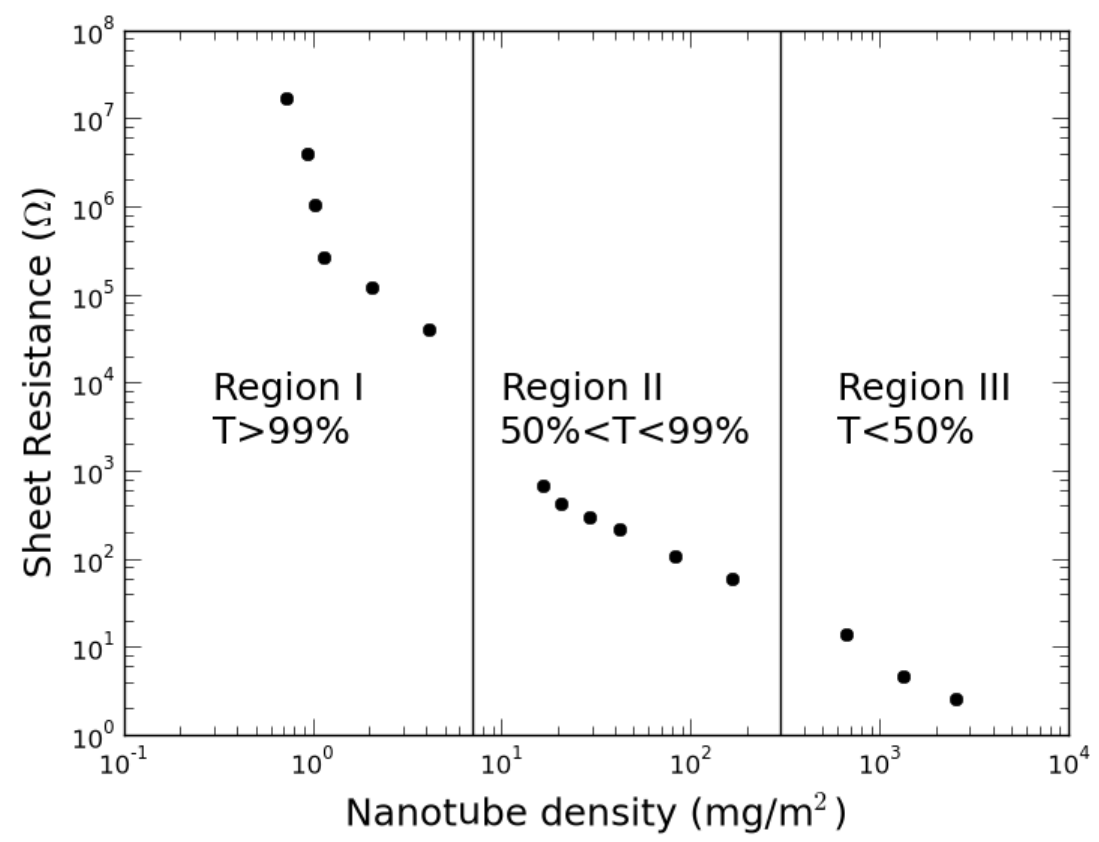

Figure 3.4: Different macroelectronic zones separated by nanotube density. Region I corresponds to thin film transistors, region II to transparent electrodes and region III to supercapacitors and batteries. Figure from [27]

Not all connected paths in the SWNT network carry current when the starting material is a heterogeneous mix with majority semiconducting tubes. However, there are certain situations where semiconducting nanotubes are part of the current-carrying network. In such cases, the figure of merit, $\sigma_{D C} / \sigma_{A C}(\beta)$, can be used to compare samples with the same M:S ratio but a different proportion of activated semiconducting tubes. If the percolation measurements have accompanying optical measurements, Eq. 3.4 [66] can be used to fit the data and obtain a value for $\beta$. The reader is referred to a recent review [67] that discusses $\beta$ in the context of nanowire networks.

$$
\% T=1.0+\frac{188.0^{-2.0}}{\beta R} \times 100
$$


Figure 3.5 shows an example of percolation data from two sources fitted with Eq. 3.4. The data plotted in black is from films produced from arc discharge SWNTs from Carbon Solutions (see Table 3.2 [45]. The data plotted in purple is from the data published in an earlier study 52] that led to this $\mathrm{PhD}$ research. The films were fabricated by drop-casting but using the same SWNT dispersion strategy utilised in this study (Chapter 4.2.1). The M:S ratio of the starting material is the same for both samples but the fitted values of $\beta$ are 6.3 and 0.04 for the arc-discharge and HiPCO films respectively. Part of the reason for this discrepancy can be related to the activation of semiconducting tubes by intrinsic doping.

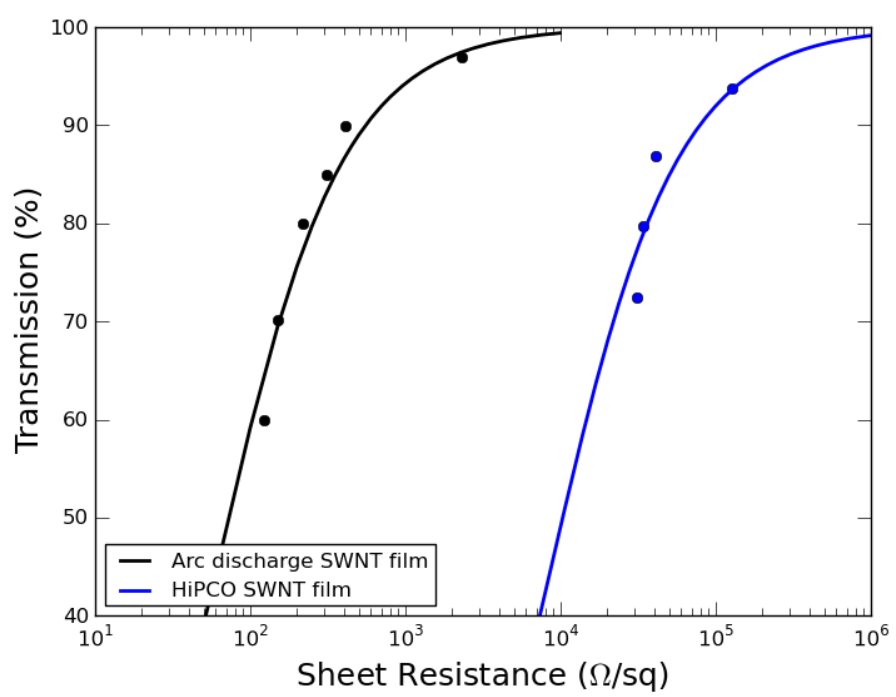

Figure 3.5: Comparison of pristine films produced using HiPCO [52] and arc discharge (Carbon Solutions [45] SWNTs respectively.

In order to differentiate between ambient doping by atmospheric oxygen [68] and external chemical dopants like nitric acid, doping is classified into two types in this work: intrinsic and extrinsic. Both types of doping have a similar effect on the SWNTs: pushing the fermi level towards the valence van Hove Singularity. This movement of the fermi level, or p-doping, can be up to $0.4 \mathrm{eV}$ [69]. Thus, semiconducting SWNTs valence bands that are up to $0.4 \mathrm{eV}$ from the fermi level prior to doping can be activated even in ambient conditions. Not all SWNTs are doped however. There is a distinct diameter dependence for doping since the transition energies themselves are dependent upon diameter.

Figure 3.6 a shows the Kataura plot for $E_{11} / 2$, from $0 \mathrm{eV}$ to the valence van Hove singularity. The doping level of $0.4 \mathrm{eV}$ is marked as a horizontal line and the diameter distributions are highlighted in pink and green for HiPCO and arc-discharge tubes (HiPCO tubes have a mean diameter of $1.05 \mathrm{~nm}$ while arc discharge tubes have a mean diameter of $1.4 \mathrm{~nm}$ ). Since the arc-discharge tubes have $E_{11} / 2$ below $0.4 \mathrm{eV}$, their Fermi levels can be easily pushed into the first valence VHS or beyond. Figure 3.3a shows that even semiconducting tubes have non-zero 


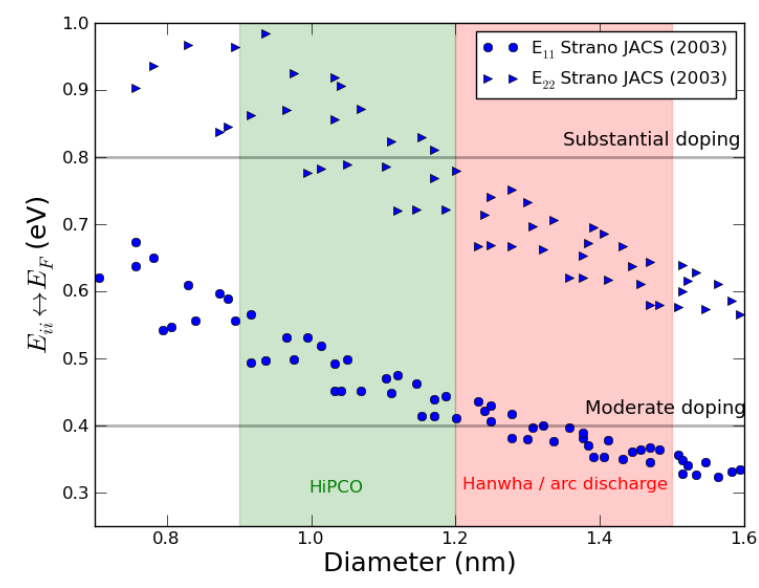

(a) Doping effects

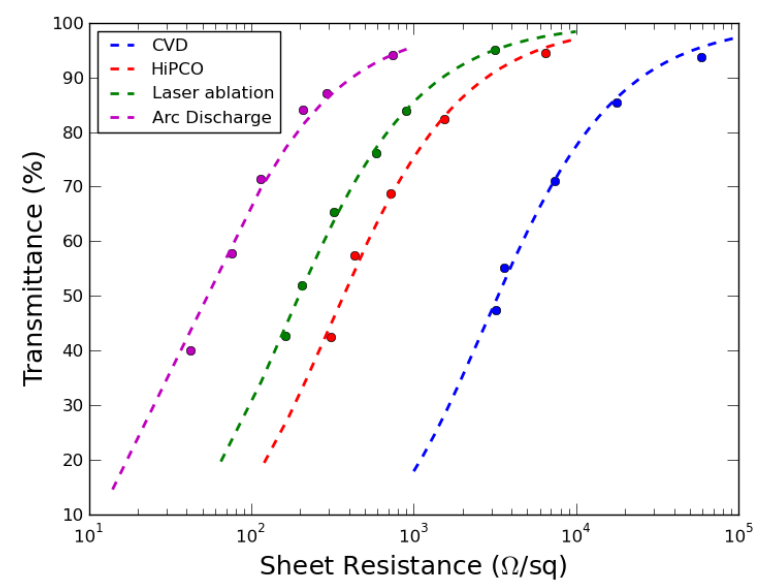

(b) Optoelectronic properties by SWNT type

Figure 3.6: (a) Empirical Kataura plot extablished by Strano [60]. The plot qualitatively highlights the diameter distributions of HiPCO and Hanwha arc disharge tubes. N.B The mean diameter of Carbon Solutions SWNTs is close to the Hanwha tubes: $1.4 \mathrm{~nm}$ (b) Optoelectronic properties based on intrinsic doping suggesting a distinct trend to higher $\beta$ values for films fabricated from larger diameter tubes.

density of states beyond the first vHS. The HiPCO tubes are too far away in energy to be influenced by ambient doping; the window for ambient doping begins for nanotubes with diameters above $1.3 \mathrm{~nm}$. Since the majority of arc-discharge tubes have diameters larger than this values. A significant fraction of the connected pathways in a network of arc-discharge tubes can carry current, thereby enhancing $\beta$ compared to the HiPCO films. Similar trends based on diameter-dependent ambient doping can be seen in Figure 3.6b. The average diameters for CVD, HiPCO, laser ablation and arc discharge SWNTs are $0.8 \mathrm{~nm}, 1.0 \mathrm{~nm}, 1.2 \mathrm{~nm}$ and 1.4 $\mathrm{nm}$ respectively [70]. As the diameters get larger, the $\beta$ value begins to increase and shifts the curves towards the left. Thus, intrinsic doping is an important parameter that enhances the DC conductivity without altering the optical conductivity.

P-doping by ambient $\mathrm{O}_{2}$ is a moderate effect which leads to $\beta$ values between 6 and 13 [43, 71, 45]. However, stronger p-doping by acids like nitric acid $\left(\mathrm{HNO}_{3}\right)$ or other dopants like thionyl chloride $\left(\mathrm{SOCl}_{2}\right)$ can improve the DC conductivity manifold. Networks of $\mathrm{HNO}_{3}$-doped arc discharge tubes fabricated by Nirmalraj et al. [29] and similar films of Geng et al. [71] have $\beta$ values of 35 and 25 respectively. Strong acid doping can shift the Fermi level by $0.8 \mathrm{eV}$ [69] (as seen in Figure 3.6a (a)). This shift moves nearly two thirds of semiconducting tubes that have $E_{22}$ below $0.8 \mathrm{eV}$ into the second Van Hove singularity of the valence band (calculated using the data of Strano et al. [60]). In fact, doped semiconducting tubes make better charge carriers compared to metallic tubes due to the large density of states available within $0.8 \mathrm{eV}$ [69]. Hence, films with predominantly semiconducting tubes (fabricated from solutions with greater than $90 \%$ enrichment) can be doped such that their conductivities are comparable to films with a majority of metallic tubes [50, 72, 69]. 
For p-doping to have any effect on the macroscopic resistance, it must decrease barrier sizes. In Section 4.4, we saw that the sheet resistance in SWNT networks is governed by inter-tube barriers. Skàkalovà et al. [54] and Barnes et al. [50] have shown that acid and $\mathrm{SOCl}_{2}$ doping decreases the junction barriers in both thick and thin films respectively; the value of the $T_{B}$ parameter - extracted from the fitted FIT curve - is markedly decreased in doped films. More recently, Nirmalraj et al. [29] showed that barrier sizes are decreased almost three-fold after acid treatment. One of the mechanisms associated with reduced barrier sizes is densification of the network after doping [73].

Densification of the SWNT network occurs when solvent/extraneous molecules are desorbed from the nanotube sidewall. When present, the adsorbates prevent nanotubes from getting close enough to form bundles. When removed, the 'fluffy' network collapses bringing many nanotubes into intimate contact. This physical change was monitored in our previous study [52] and found to considerably reduce the sample resistance in two ways: decreasing barrier size and activating blocked M-S junctions. Both mechanisms owe their presence to the reduced spatial separation of bundles.

$$
\begin{aligned}
\Phi_{S B} & =\chi+\frac{E_{G}}{q}-\Phi_{M} \\
& =\left(4.1-\frac{E_{11}}{2}\right)+E_{11}-4.5
\end{aligned}
$$

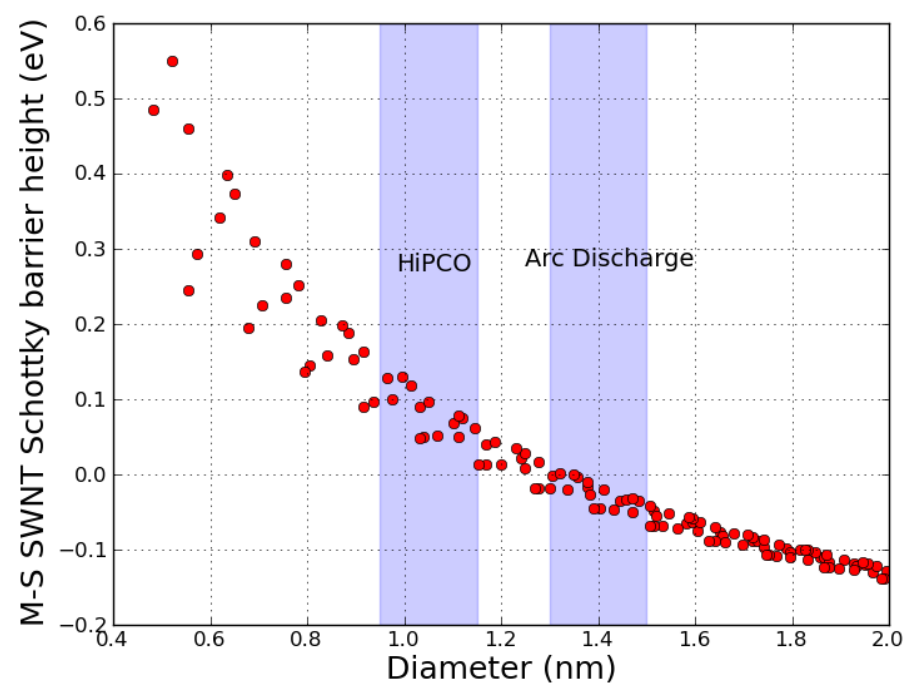

Figure 3.7: Schottky barrier heights calculated for empirical bandgap energies ( $E_{11}$ values from Strano et al. [60]) 
The novel observation of the activation mechanism only in our HiPCO films can be rationalised by considering the expected size of Schottky barriers. Eq. 3.5 shows that Schottky barrier sizes can be calculated from the $E_{11}$ transition energies (Strano et al. [60]). The parameter, $\chi$, is the electron affinity for the semiconducting nanotubes; $E_{G}$ (or, $E_{11}$ ) is the bandgap and $\Phi_{M}$ is the workfunction for metallic nanotubes. The value for $\chi$ is calculated using the workfunction for undoped semiconducting tubes while the workfunction for metallic tubes is the doped value [69]. For the illustrative example provided here, $\Phi_{M}$ is assumed to be independent of diameter. The value of the workfunction in tubes doped by ambient oxygen is given as $4.6 \mathrm{eV}$ [69], in agreement with a calculated value of $4.7 \pm 0.2 \mathrm{eV}$ [29, 74]. Figure 3.7 shows decreasing Schottky barrier size with increasing diameter. For diameters greater than $1.3 \mathrm{~nm}$, the Schottky barrier becomes negative and there is no junction barrier, equivalent to metallic-metallic junctions. Nanotubes with diameters below $1.3 \mathrm{~nm}$ have finite barrier sizes. The corresponding barrier size for the mean diameter of HiPCO SWNTs $(1.05 \mathrm{~nm})$ is around $0.1 \mathrm{eV}$, not far from the measured barrier energy of $0.2 \mathrm{eV}[52]$.

The emergence of activation over Schottky barriers in networks of HiPCO SWNTs can be inferred from Topinka et al. [51]. In thin networks, surface coverage of three times the percolation threshold, the study found that metallic pathways exist for semiconducting fractions below $67 \%$. At the same surface coverage but with a semiconducting fraction between $67 \%$ and $86 \%$, inter-tube connections are blocked by Schottky barriers and the network is highly resistive. Such a blocked network could be fabricated from nanotube samples that have a M:S ratio of 1:2 such as HiPCO nanotubes (Table 3.2). Thus, the activation of these blocked junctions are noticeable in the temperature dependence data.

$$
G=G_{0} \exp \quad-\frac{T}{T}^{\frac{1}{d+1}^{\#}}+G_{m}+G_{1} \exp \frac{-E_{a}}{T}
$$

Eq. 3.6 and Figure 3.8 show the underlying conduction processes in HiPCO thin films split into three components: a very small degree of pure metallic percolation $\left(G_{m}\right)$, dominant VRH conduction from $5 \mathrm{~K}$ to $200 \mathrm{~K}$ (first term in Eq. 3.6) and, Schottky barrier activation above $200 \mathrm{~K}$ (final term in Eq. 3.6] [52]. The small degree of metallic percolating paths was inferred from the observation of a non-zero value of conductance below $5 \mathrm{~K}$. Instead of the conductance decreasing monotonously to zero (as expected for VRH) the conductance reached a saturation point, a feature reminiscent of the tunneling limit of the FIT mechanism. This feature coupled with the activation of Schottky barriers was only observed in annealed samples. Combined with a parallel study of the G-mode before and after annealing, the main physical change was found to be increased bundling or collapse of the network following the removal of remnant solvent. 


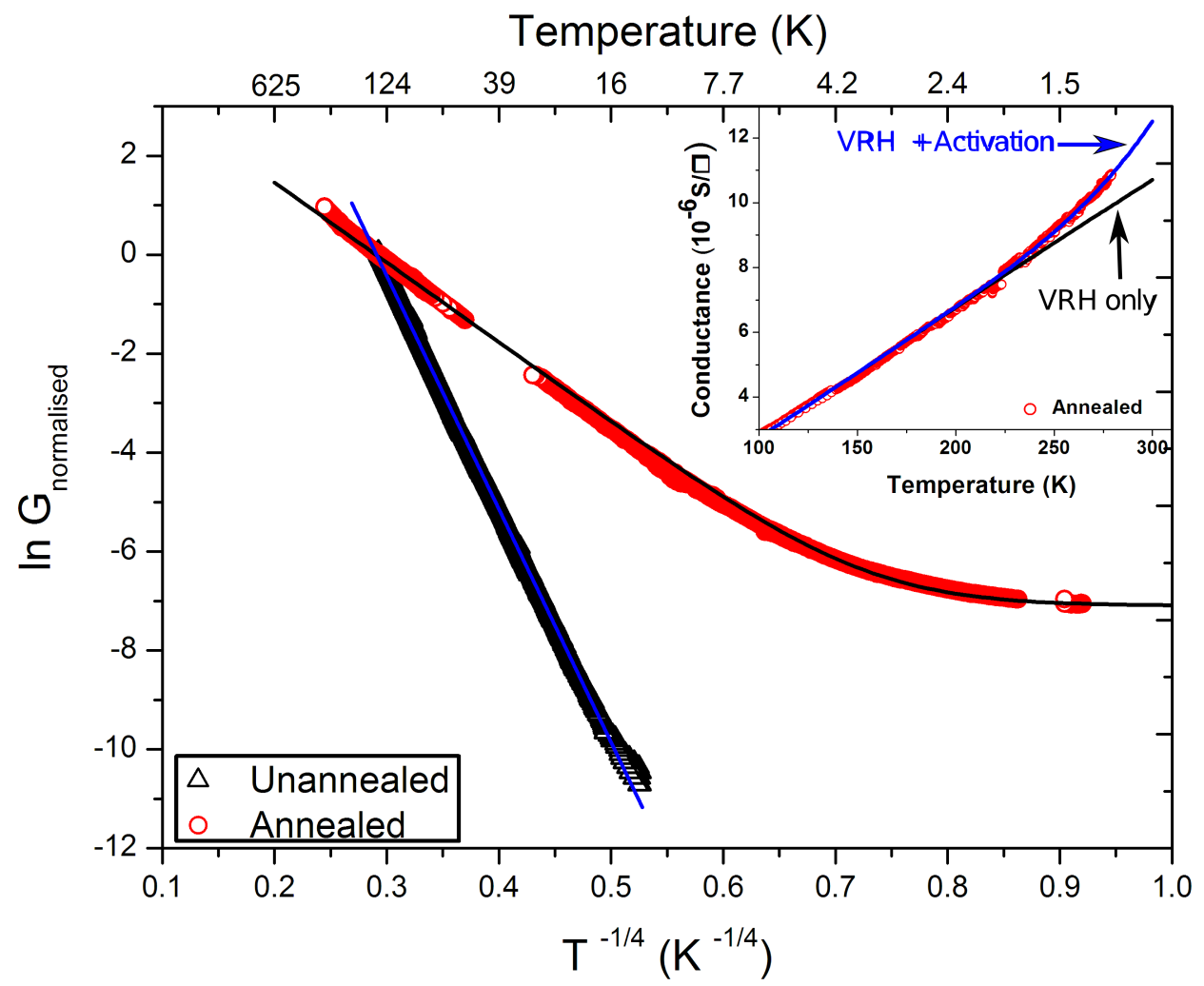

Figure 3.8: Conduction processes in annealed and unannealed HiPCO films [52].

\subsection{Correlating percolation and charge transport models}

The nature of the metallic tunnelling at low temperatures suggests that the dominant VRH conduction is not restricted to metallic-metallic components. The small number of pure metallic pathways could be another reason for the very low $\beta$ value observed in our HiPCO films (Figure 3.5). A simple question follows this observation: will an increase in the number of pure metallic pathways change the underlying properties of the three transport mechanisms identified in Eq. 3.6? Other connected questions follow this: will increasing metallic percolation change the barriers or alter the contribution of activation over Schottky barriers? Will an increase in metallic pathways induce a changeover from VRH to FIT? A study on thin and thick networks (buckypaper) by Skàkalovà et al. [75] showed that VRH is the prevalent conduction mechanism in thin networks (with optical transparency between 50\% and 90\%) but FIT dominates in buckypaper. However, this experimental study only demonstrates the existence of a changeover using extreme cases e.g. thin network vs. thick. The gradual transition from VRH to FIT by varying the extent of metallic percolation paths remain unknown.

A percolation strategy (described in Section 3.3 can be used to fabricated samples with a gradual variation in metallic percolation paths. Ideally, the percolation trace is linked to the type and size of junction barriers in the network. Various points along both the power law and linear regimes in particular. However, this approach requires the percolation trace to be scalable and, that the two regimes are distinguishable. Both these features are too sensitive upon 
the deposition characteristics and will be difficult to attain without a high degree of control. Instead, we begin by testing the idea of uniting the pictures of percolation and charge transport mechanisms with the simplistic representation of the percolation model: sheet resistance. In Chapter 4, we present a controllable deposition process with in-situ monitoring of the sheet resistance to fabricate samples with resistances spanning almost two orders of magnitude (300 $\mathrm{k} \Omega$ to $8 \mathrm{k} \Omega$ ). The percolation traces and mechanisms of charge transport within the samples, identified from temperature-dependent measurements of resistance, are discussed within the same chapter.

\subsection{Sample diagnostics using Raman spectroscopy}

This particular test of correlation between sample resistance and junction barrier size is only possible for pristine SWNT networks. Our strategy for obtaining pristine networks relies on SWNT dispersions using volatile solvents which can be removed by heating either during or post-deposition. This approach leaves a pristine network and also results in a physical change: network densification [52]. However, connected physical and chemical processes related to the fabrication process are still unknown e.g. atmospheric oxygen doping or de-doping and increase in bundle size. Thus, we present a parallel Raman study on the local chemical and physical environments in SWNT networks for our chosen fabrication process using a spatial averaging strategy. The spatial averaging strategy provides automated deconvolution of a single composite for a high volume of data. The parameters of the component peaks are analysed using simple statistics. Results of the statistical analysis can be compared with other samples or compared post- and pre-treatment to isolate significant effects that are morophology-independent, i.e. they affect the entire sample.

A corresponding study on temperature effects (both ambient and local) is carried out in order to isolate real physical/chemical effects from local heating; which is problematic in bundled SWNTs [76]. For this study, we present a second tool for deconvoluting Raman spectra; one that uses higher order derivatives to accurately deconvolute spectra with poorly resolved features. This technique can be utilised to fit complicated spectra containing many component modes e.g. radial breathing modes from bundled nanotubes.

The use of Raman spectroscopy both as a diagnostic tool to study samples and as a probe of fundamental properties (e.g. the effect of temperature) is covered in some detail within this thesis. Chapter 5 presents a brief review of the radial breathing modes (the focus of this study) and expands on the high resolution and spatial averaging strategies highlighted above. The chapter finishes with a characterisation of the thin film fabrication process. The following Chapter 6 details the temperature dependence of the radial breathing modes both under ambient and local heating. 


\section{Chapter 4}

\section{Charge transport mechanisms in SWNT networks}

\subsection{Introduction}

This chapter details a study that unifies percolation and charge transport models. As outlined in Chapter 3.4, the unification is based upon the controllable fabrication of samples at defined points along the percolation curve. Section 4.2 describes such a fabrication process. The homogeneity of the sprayed samples is characterised from microscopic measurements of nanotube density in Section 4.3. A selection of samples are studied as a function of temperature to identify the prevalent conduction mechanisms in Section 4.4 .

\subsection{Film fabrication}

At the core of this work is the development and optimisation of an experimental set up that can monitor the sheet resistance as a function of deposition time. Such a set up consists of a continuous deposition system coupled to computer logging of the sheet conductance as a function of deposition time. This apparatus enables the fabrication of films at defined points along the percolation curve. The film fabrication process is comprised of two parts: creating nanotube dispersions and depositing films from these dispersions.

\subsubsection{Nanotube dispersion}

Fabricating SWNT networks from solution is a widely used technique. It has the advantage of being cheap and quick, in comparison to more expensive and time consuming techniques like CVD. Creating the solution - technically, dispersion - has a number of facets. Not only must the component solvents break apart SWNT bundles, but a careful choice of solvent can result in selective enrichment of SWNTs by diameter, chirality or electronic type. 
The SWNT dispersion typically has two components: a solvent matrix and an adsorbant. In some cases, the solvent matrix also acts as the adsorbant [77]. The adsorbant is the key component that adsorbs on the SWNT sidewall and prevents agglomeration of nanotubes. The adsorbed molecules prevent re-bundling of the SWNTs through steric hinderance. Not all solvents can achieve this. Ethanol, for example, is a very poor adsorbant for HiPCO SWNTs. A dispersion can be created by sonication but the dispersion degrades within minutes. The SWNT material flocculates into a fluffy mass and precipitates out of solution. In the presence of good adsorbants, dispersions can remain stable for extended periods of time.

The procedure for creating high quality SWNT dispersions requires both sonication and centrifugation. Sonication exfoliates the bundles followed by adsorption of the adsorbant on the tube walls [78]. Since large bundles still remain in the solution, centrifugation is often used to phase separatre large bundles from the well-dispersed solution. However, centrifugation is often a lengthy process and also removes longer tubes in addition to large clumps.

The most common nanotube dispersion uses water as the solvent matrix and a surfactant, sodium dodecyl sulphate (SDS), as the adsorbant [79]. The major issue with this dispersion is the intensive processing required to remove the adsorbed SDS. The typical procedure utilises several iterations of washing to remove traces of surfactant. However, the washing can be ineffective and additional treatment is required to fully remove the SDS [71]. Similar issues arise with other bulky surfactants [80]. It is vital that surfactant traces are removed. When present, sheet resistances are high due to large junction barrier size bought about by the spatial separation of nanotubes [52].

The two dispersion components used in this study - tetrahydrofuran (THF) and n-butylamine - have been chosen so that they can be removed by heating. N-butylamine is considered a 'surfactant' due to its functionality, though it is not a surfactant by definition. From our own experiments and those carried out by Maeda et al. [81], we can infer that the amine acts as a surfactant: it adsorbs on the nanotube walls during sonication to form a coating that is soluble in the solvent THF. The adsorbed layer also acts as a spatial barrier that prevents nanotube aggolmeration [52]. A preliminary Raman study of this dispersion system found that the 1:2 ratio of metallic to semiconducting tubes found in the HiPCO samples (Table 3.2) is preserved in solution [82]. That is, there is some evidence that the system is involved primarily in debundling and there are no complications of having to account for selective enrichment.

The evidence for complete solvent removal by heating (also referred to as annealing) is obtained from thermogravimetric analysis (TGA) in parallel with differential scanning calorimetry (DSC). Figure 4.1 shows both the TGA and DSC curves for the solvents used in in this PhD study. The low temperature region shows peaks at 66 and $96{ }^{\circ} \mathrm{C}$. The first peak can be as- 
sociated with the boiling point of THF and the second, broad peak with the boiling point of n-butylamine and an impurity (likely water). The two peaks seen at high temperature in the DSC are associated with combustion since the positive heat flow indicates it is an exothermic reaction. The higher temperature peak at $439{ }^{\circ} \mathrm{C}$ is due to combustion of the HiPCO nanotubes 83. while the peak at $267^{\circ} \mathrm{C}$ could be early onset combustion bought about by extrinsic doping (Chapter 3.3) of the nanotubes by the solvent [84], or burning of an impurity in the solvent. In our earlier work, a higher temperature anneal at $320^{\circ} \mathrm{C}$ was necessary to improve the optical transparency. This effect was attributed to the presence of impurities in the n-butylamine solvent. Annealing the films to $100{ }^{\circ} \mathrm{C}$ did not improve the optical transparency. The impurity could also be identified visually as unannealed films had a cloudy look and correspondingly poor optical transparency of $\sim 60 \%$ [82. Hence, all work carried out in this $\mathrm{PhD}$ utilised a new batch of n-butylamine purchased from Sigma Aldrich. In this batch, the impurity is absent and high optical transparencies were achieved even after annealing at $110^{\circ} \mathrm{C}$ (Section 4.3 ).

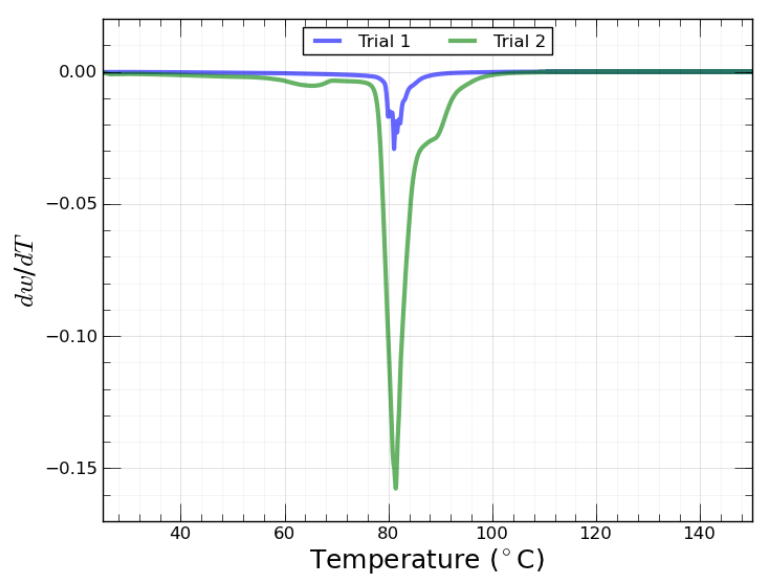

(a) Differential thermogram: zoom to $150{ }^{\circ} \mathrm{C}$

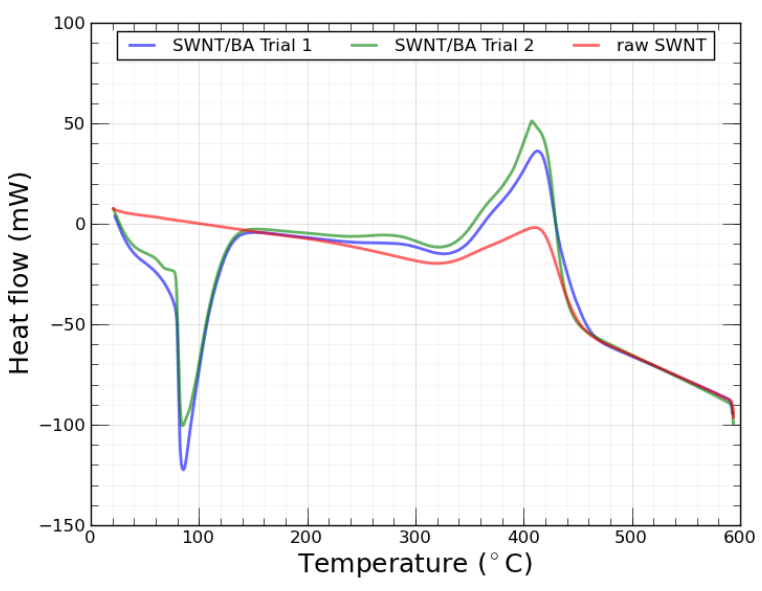

(b) Differential scanning calorimetry

Figure 4.1: Differential thermogram (a) and differential scanning calorimetry (B) for SWNTs dispersed in a THF/Butylamine (new batch) solvent system.

Nanotube dispersions were prepared in a similar method to our earlier study [52]. A small quantity of raw HiPCO SWNTs ( $\sim 1 \mathrm{mg})$, purchased from Carbon Nanotechnology Inc., was added to a 7:1 ratio of THF to butylamine (total volume of $50 \mathrm{~mL}$ ). The mixture was sonicated until the solution turned a translucent grey with no visible clumps (typically 2 hours). As we required no selective enrichment (however inadvertent) and a high weight concentration of SWNTs (for faster film deposition), the centrifugation process was forgone. Any visible lumping was remediable by the addition of n-butylamine and sonication for several minutes. With this method, percolation threshold could be established within several minutes. However, bundle sizes in solution are unfiltered and the larger bundles were responsible for frequent blocking of the nozzle (Section 4.2.2). Successive iterations of this experiment will be trialled with mildly centrifuged dispersions to prevent large bundles from disrupting the spray. 


\subsubsection{Film deposition}

A variety of methods can fabricate SWNT networks from solution; from transfer-printing 85 to spraying [86, 87] to inkjet printing [88]. The optoelectronic ratio is impervious to different deposition techniques since the components of a random network have a high degree of freedom. Figure 4.2 pools data from the literature for Hanwha arc-discharge and CoMoCAT nanotubes. For the Hanwha arc discharge tubes, prepared from similar dispersions but deposited differently (airbrushing vs. vacuum filtration) in two independent studies, the ratio is around 10. A separate study of films prepared from CoMoCAT tubes via airbrushing and vacuum filteration also share a very similar optoelectronic ratio.

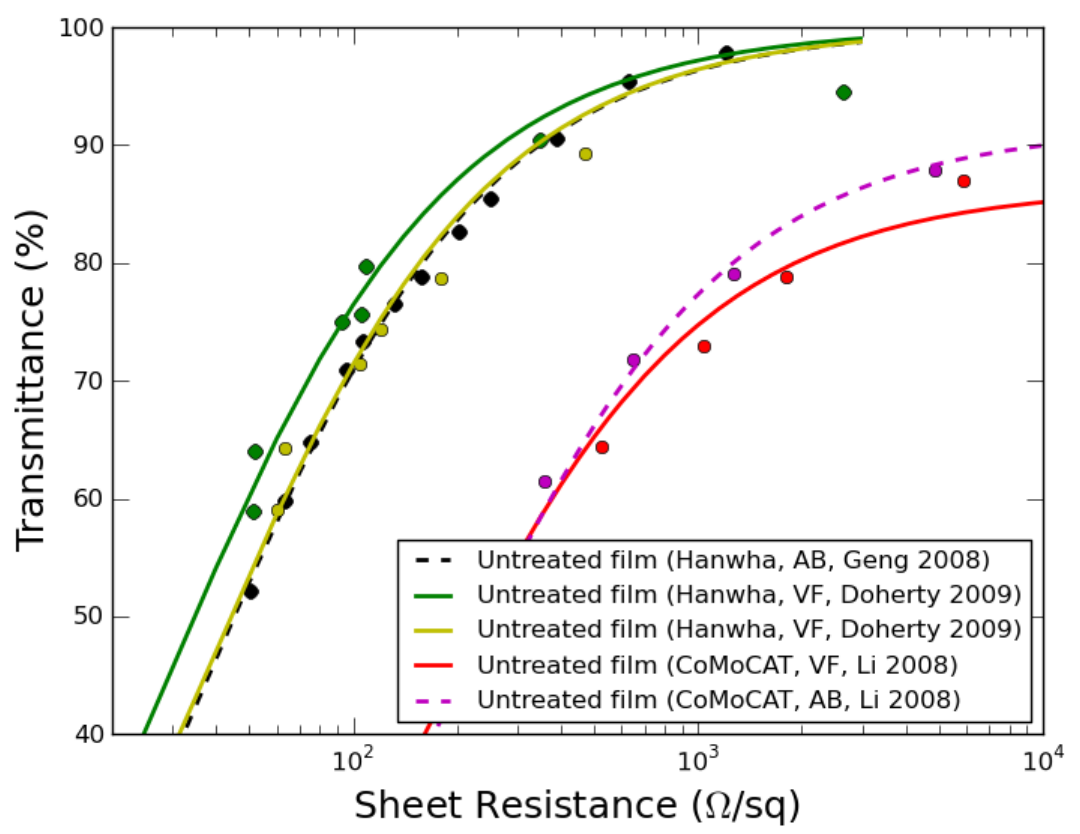

Figure 4.2: Optoelectronic properties for SWNT films fabricated by airbrushing and vacuum filteration. The data was extracted from several independent studies [89, 73, 43].

In this work, two spraying techniques were employed: airbrushing and ultrasonic nozzle spraying. Airbrushing was used at the beginning of the study to test the feasibility of the method before moving to a more sophisticated ultrasonic spraying apparatus. The set up for in-situ monitoring and control of SWNT deposition was essentially the same for spraying with an airbrush or an ultrasonic nozzle. The basic components were: electronics and logging equipment, a sample mount and controllable delivery of solution. The electronics comprised of two multimeters (one for recording temperature and the other for recording the sample resistance), a basic measurement circuit and a computer running a simple LabView program for logging data. The sample mount differed for the two spraying techniques but was an essential requirement for monitoring the substrate temperature and holding the sample in place electrically and, mechanically in the case of airbrushing. A syringe pump was used to deliver the solution 
to both the airbrush and the ultrasonic nozzle.

\section{Health and safety}

Before describing the spraying apparatus in detail, we briefly list the safety precautions necessary for spraying aerosols of nanomaterials and corrosive solvent (n-butylamine). All spraying was carried within a fumehood with the doors pulled down for maximum venting. Shields were also used as an additional safety measure. The shield for the airbrush was a large perspex halfbox that safe-guarded against accidental exposure to the aerosol. The spray from the ultrasonic nozzle was shielded completely by a glass cylinder (shown in Figure 4.4). The experimenter (myself) adhered to strict dress protocol which included laboratory coat, closed-toe shoes, high risk gloves, eye glasses and a face mask. The total amount of sprayed material in a single deposition experiment was tens of micrograms as only a couple of millilitres of solution were utilised.

\section{Ultrasonic nozzle}

While the airbrushing apparatus allowed for a proof-of-concept experimental set up (Appendix 11), the detrimental effects of strong surface cooling and mechanical damage to the contacts did not provide reproducible, high quality samples. The large thermal load due to surface cooling by the carrier gas was circumvented by moving to a spraying system equipped with an ultrasonic nozzle. The transducer within the nozzle produces a fine mist of solution droplets with no dependence on the air pressure. Thermal resistance between the hotplate and substrate was minimised by using a thick copper base for the sample mount and a thermally conducting sapphire substrate.

The ultrasonic nozzle resolved the major issues with the airbrush but gave rise to a new issue: the spray cone was considerably smaller $(\sim 2 \mathrm{~mm}$ in diameter at typical working conditions). This limitation necessitated clean room fabrication techniques to produce electrodes that complied with the size of the spray cone. Details of the photolithography process to fabricate electrodes are highlighted in Chapter 2.4.1. Contacts to these substrates were made using a Kulicke and Soffa 4124 gold ball bonder. The advantage of ball bonding to contacts were manifold. For one, the high temperatures during deposition and subsequent annealing at 320 ${ }^{\circ} \mathrm{C}$ (for some samples) were no longer an issue since the ball bonded contacts are pure gold metal, and stable to very high temperatures. The ball bonded contacts were also extremely robust and did not need replacement. In most cases, a single sample could be tested multiple times without any damage to the contacts.

The substrate was connected in series with a $9 \mathrm{~V}$ battery and a resistor $(1 \mathrm{M} \Omega)$ which acted as a load when the substrate was not covered by a percolating network. Two Keithley multimeters (models 195A and 196) were used to $\log (\mathrm{i})$ voltage from a resistor that is calibrated as a ther- 
mometer (PT-1000) and (ii) voltage across the $1 \mathrm{M} \Omega$ resistor. The multimeters were connected to a laptop, via GPIB, running a LabView program. Within the program, a simple formula was used to convert the voltage from the resistor to sample resistance (Eq. 4.1 and Figure 4.3). The sample conductance as a function of time was plotted on the front panel of the program for visual control of the deposition process. When a battery was used, the supply voltage was always measured at the start of a deposition experiment and manually updated in the program.

$$
R_{\text {sample }}=\left(V_{\text {supplied }}-V_{R}\right) /\left(V_{R} / 1 \times 10^{6}\right)
$$

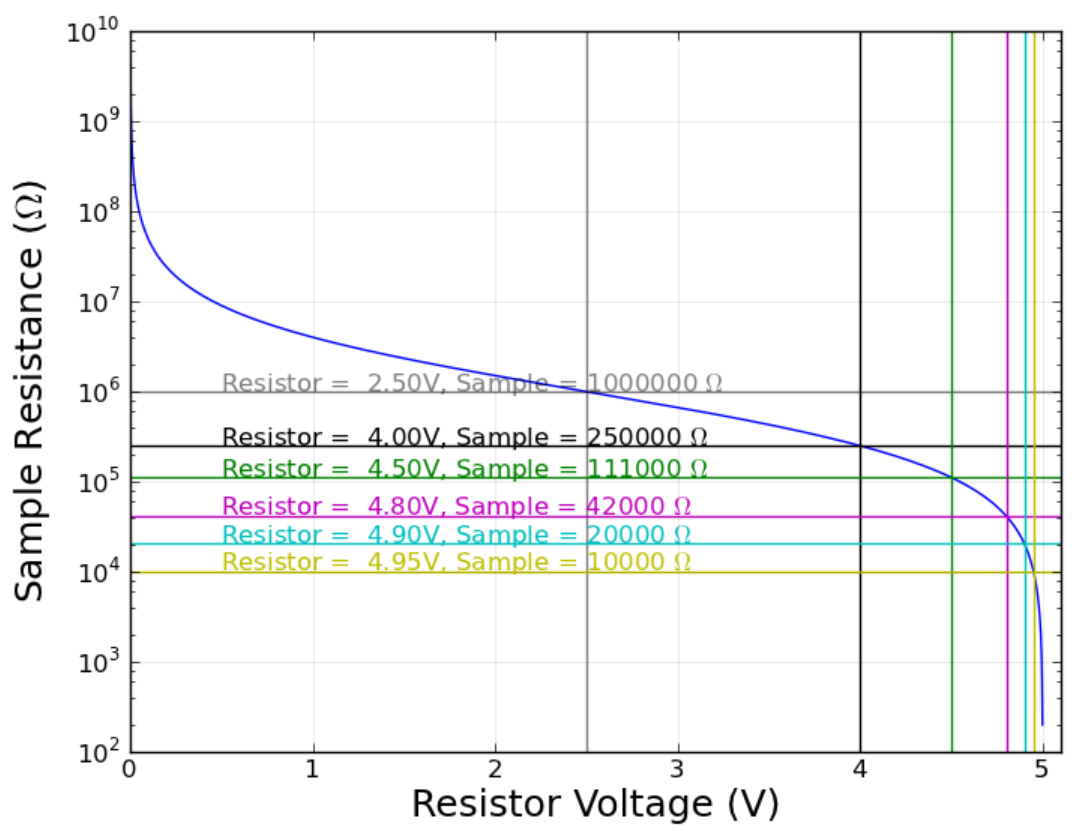

Figure 4.3: Sample resistance as a function of measured resistance

The final experimental set up is shown in Figure 4.4. Changes from the initial set up include a new syringe pump for accurate flow monitoring, the ultrasonic nozzle, a standard laboratory hotplate to set the substrate temperature and a new sample mount. The new sample mount was a particularly useful addition to the apparatus as the connections to the sample were mechanically robust and physically decoupled from the sample, an improvement upon the original mechanical clamp which caused considerable damage to the gold contacts. Figure 4.5a shows a photograph of the mount. The large copper base took some time to heat but once heated, the substrate temperature could be maintained to within a few degrees (Figure 4.6a) despite the poor feedback to the heating control and strong cooling from the fumehood - which created long gaps of no heating and short bursts of strong heating. The connection from the gold pins to the sample was made via small pillars filled with solder (Figure 4.5b). The leads from the sample were soldered to the pillar and the gold pins were mechanically secured to these pillars. From the gold pins, wire leads were directed to a electrical connection box before reaching the 


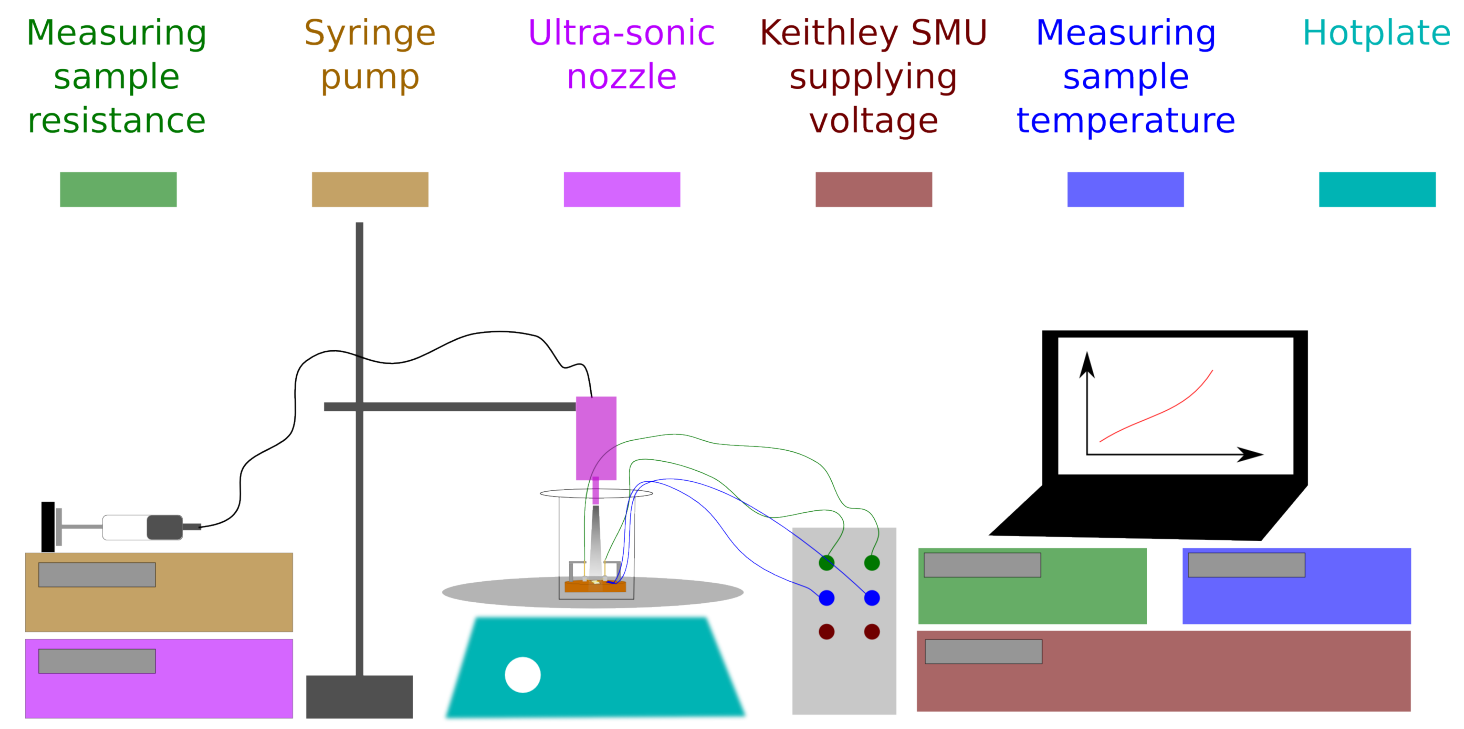

Figure 4.4: A schematic showing the experimental set up with an ultrasonic nozzle.

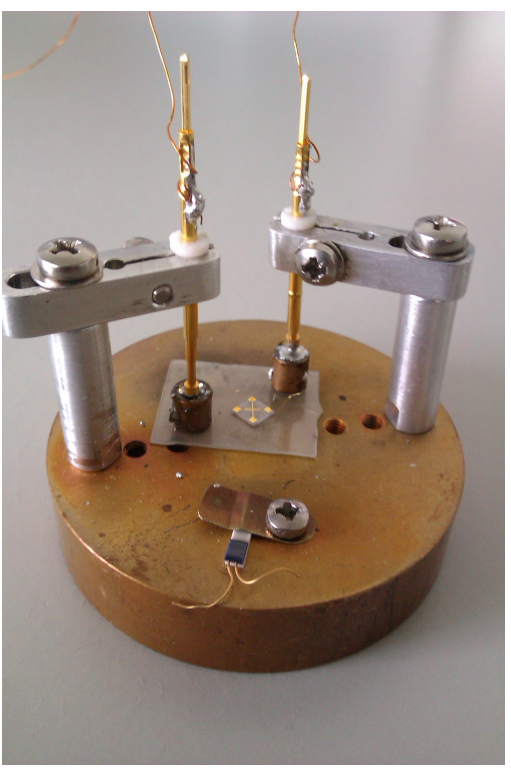

(a) Sample mount

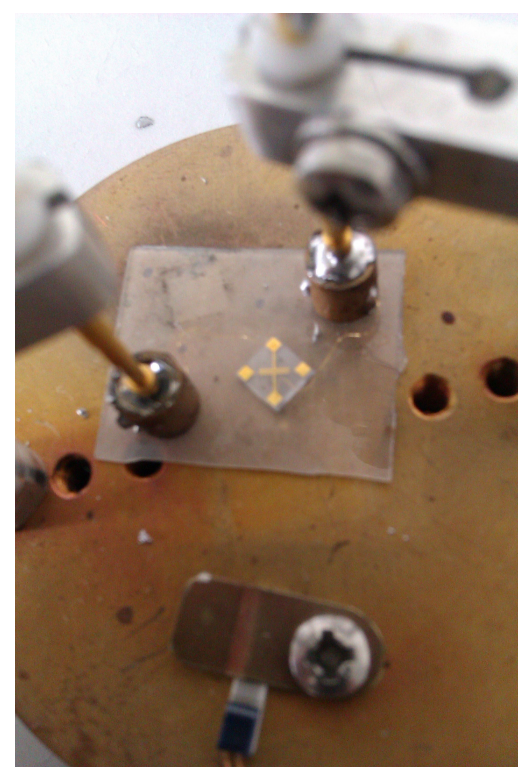

(b) Closeup of connection to sample

Figure 4.5: The sample mount used with the ultrasonic nozzle

multimeter. The electrical circuit for this setup was largely unchanged; only the battery was swapped with a source measure unit - a more reliable and importantly, adjustable, source of voltage.

Samples were prepared by following a general methodology:

- Set hotplate to temperature that results in a final substrate temperature greater than 100 ${ }^{\circ} \mathrm{C}$.

- Start syringe pump at high flow volume to deliver solution to the ultrasonic nozzle. Reduce flow to $(50 \mu \mathrm{L} / \mathrm{min}$ with a $3 \mathrm{~mL}$ syringe $)$ once the solution reaches the nozzle. 


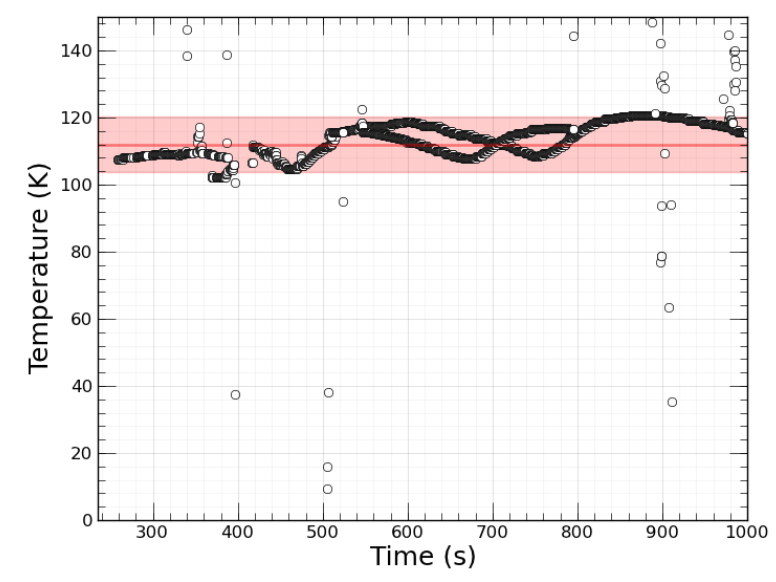

(a) Temperature during spraying

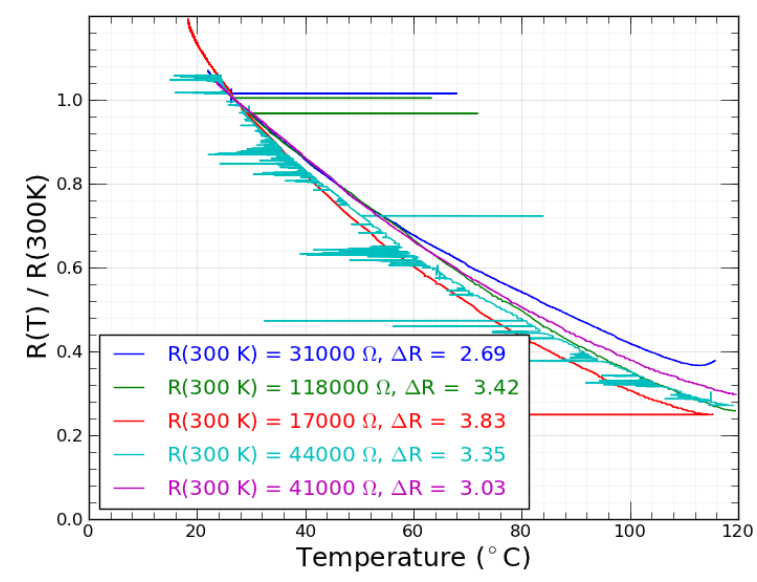

(b) Cooling curves

Figure 4.6: (a) Temperature during spraying from two different runs. Average temperature is around $112 \pm 8{ }^{\circ} \mathrm{C}$. (b) Cooling curves for 5 different samples.

- When the substrate temperature has stabilised, operate the ultrasonic nozzle (91\% power) and syringe pump simultaneously to begin spraying.

- Begin logging sample resistance once spray is stable.

- Stop spray when the sample resistance reads the desired value* $\mathrm{N}$. $\mathrm{N}$ is the correction factor due to the enhancement of conductance (relative to room temperature) at an elevated temperature of $112{ }^{\circ} \mathrm{C}$. Figure $4.6 \mathrm{~b}$ shows that the average resistance at $20{ }^{\circ} \mathrm{C}$ is around 3 times the value of the resistance at the deposition temperature.

While the general methodology is simple enough, a few precautions were necessary. For example, the spray had to be carefully monitored during deposition. Though the ultrasonic nozzle was more reliable than the airbrush, day-to-day variations in the dispersion often lead to blockages at the nozzle and occasional discharge of a large amount of blocked material. The discharge was ruinous to film quality and any sign of imminent discharge required that the deposition be stopped and the blockage dealt with. Otherwise, minor blockages were fairly common and resulted in a spray-sensitive percolation trace. The blockages caused intermittent spraying and a inconsistent deposition rate. The high weight concentration of SWNTs combined with unfiltered bundle sizes in solution were the likely cause behind the inconsistent spraying. In future experiments, we plan on optimising the SWNT solution (via. dilution or centrifugation) for more consistent spraying.

As seen in the previous section, the sample resistance as a function of deposition time could be roughly explained by the percolation model. To date, it is the clearest percolation curve collected from an in-situ experiment and offers some insight for that particular sample. The link between the in-situ percolation trace and the model of conduction was one of the goals of this project. However, the obtained traces from the final set up were found to be strongly 
coupled to the spray. Since the spray was not constant, the amount of material deposited per unit time was highly variable and the resultant curves unreliable for quantitative analysis.

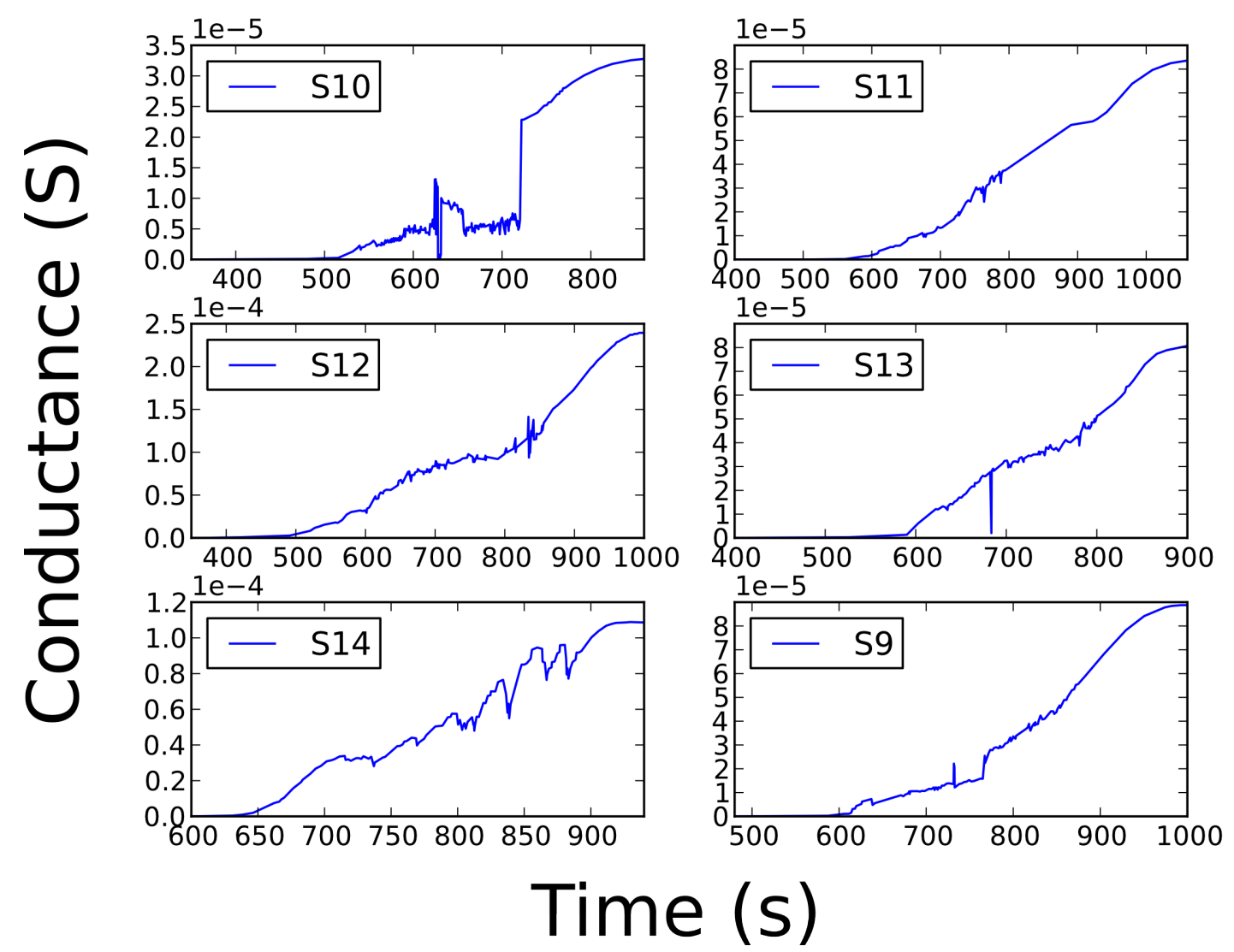

Figure 4.7: Percolation traces during deposition.

Figure 4.7 shows the sample conductance as a function of deposition time for several samples. There are qualitative similarities between some samples, particularly samples S11 - S14. The full percolation for one of the samples (S12) bears a strong resemblance between the qualitative shape of the curve with that of Figure 11.2c. While the percolation threshold was clearly identifiable, the percolation regime (where the power law holds) was difficult to determine. Figure 4.8 shows that different linear regions exist within the data. When only the first $60 \mathrm{~s}$ after percolation is fit, the power law exponent is twice the value expected for 3D percolation. When the power law fitting is extended for the full dataset, the percolation exponent drops in value but the fit quality near the percolation threshold is poor.

Even a brief analysis of the percolation traces obtained using the ultrasonic nozzle is sufficient to show that the deposition conditions need considerable improvement before the percolation trace can be analysed similar to Figure 11.2. Thus, the initial idea of fabricating samples at different points along the percolation curve was only possible at a qualitative level in the work. That is, the sample could not be associated clearly with the percolation, linear or transition (between percolation and linear) regimes. But, samples could be fabricated in a 


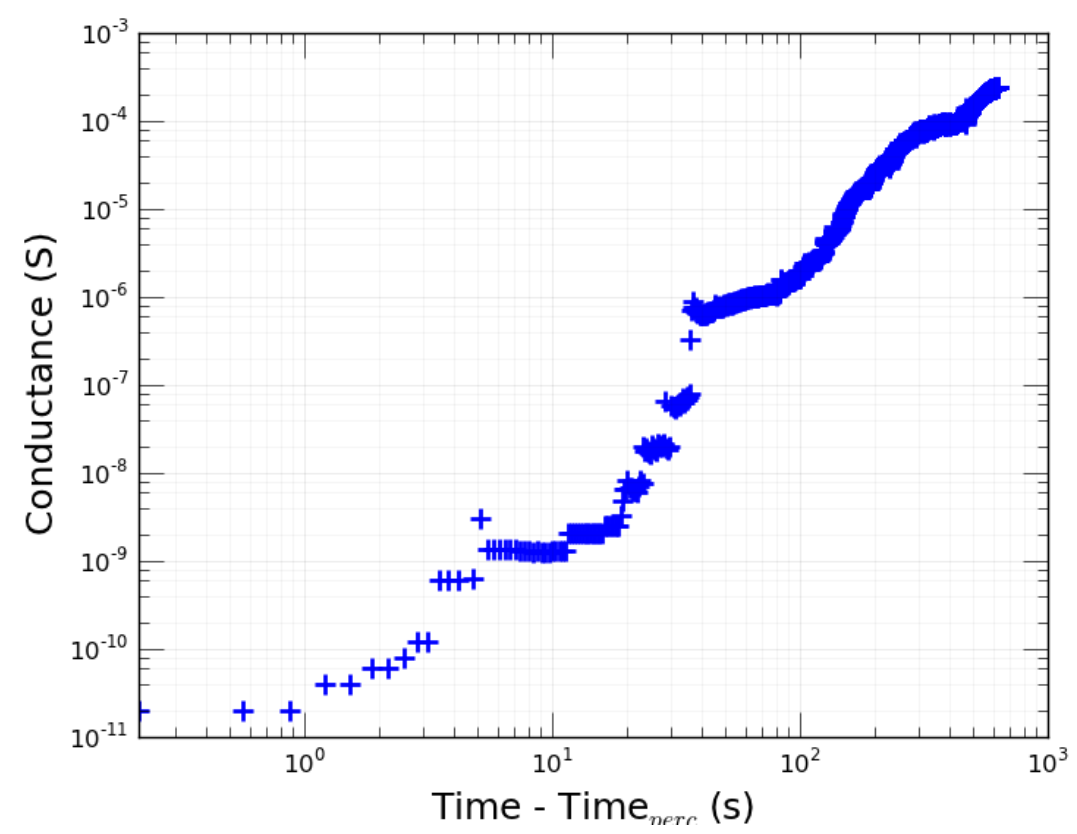

Figure 4.8: Log plot of the percolation trace for sample S12.

controllable manner as a function of deposited material. In this study, samples with resistances evenly spaced between $300 \mathrm{k} \Omega$ to $8 \mathrm{k} \Omega$ were studied (Table 4.2 ).

\subsection{Spatial homogeneity of sprayed SWNT networks}

The optical transparency of SWNT thin films is typically used as a gauge that determines the suitability of the film as a transparent conductor. The metric, $\beta$, dictates the conducting efficacy vs. optical transparency (Chapter 3). The higher the value of $\beta$, the better the transparent conductor. Here, we use the microscopic optical transparency to evaluate the homogeneity of films fabricated by the deposition process described in Section 4.2.2.

The setup for measuring microscopic optical transparency is effectively a Raman spatial mapping measurement. Since the aspect ratio of the electrodes is quite large, the map was set up such that the laser spot tracked from the top end to the bottom at the centre of the electrode gap (Figure 4.9a). As a continuous scan option was not available with the mapping stage, the map was set as a step scan with 1 s at each step and a $10 \mu \mathrm{m}$ step size. A power meter (Thor Labs PM100 with a silicon detector) was attached to the bottom of the microscope slide using double sided tape. The active area of the detector was much larger than the sample. The spatial response of the detector was found to be flat from the calibration measurement. The transparency at a given spot was calculated as: $P_{\text {meas }} / P_{\text {calibration }}$, where $P_{\text {meas }}$ was the measured power at the spot and $P_{\text {calibration }}$ was the power measured with a clean substrate. The $632.8 \mathrm{~nm}$ laser was used for this experiment as it was the simplest to set up. There is little variation in absorbance between $550 \mathrm{~nm}$ and 632.8 for thin films and no corrections are necessary. Since the 


\begin{tabular}{|c|c|c|}
\hline Sample & Optical transparency $(\%)$ & Resistance $(\Omega)$ \\
\hline \hline S4 & $88 \pm 5$ & 16000 \\
\hline S7 & $93 \pm 16$ & - \\
\hline S9 & $75 \pm 22$ & - \\
\hline S12 & $75 \pm 12$ & 18000 \\
\hline S17 & $84 \pm 6$ & 8000 \\
\hline \hline
\end{tabular}

Table 4.1: Spatially averaged optical transparencies for SWNT samples shown in Figure 4.9b. The resistance at $300 \mathrm{~K}$ (within the cryostat) is given for three of the samples.

substrate of choice was only polished on one side, a layer of Zeiss Immersion oil 518C between the substrate and glass was necessary to minimise specular reflection. Power data was logged continually (average collection speed of $0.15 \mathrm{~s}$ per data point) using a simple Python script.

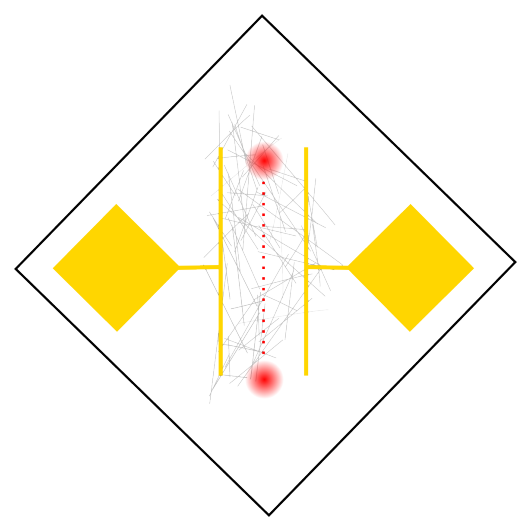

(a) Experimental setup

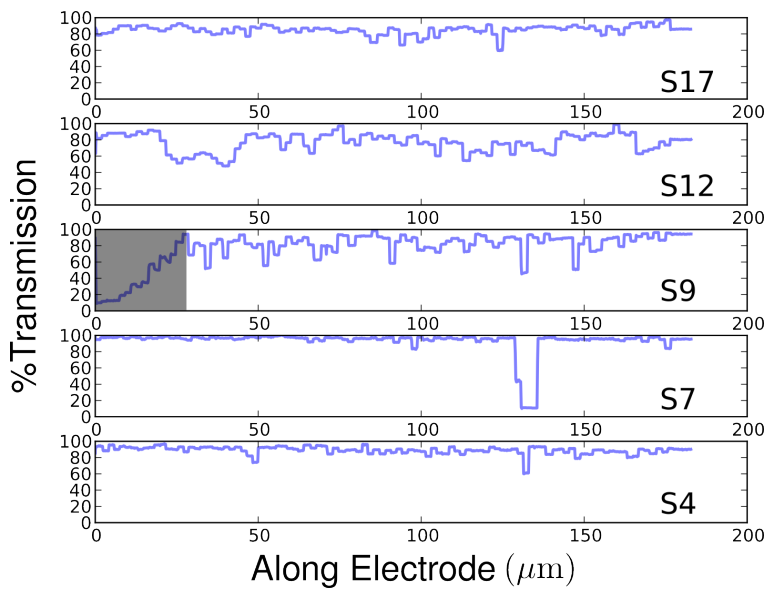

(b) Microscopic transparency

Figure 4.9: (a) Experimental setup showing that the spatial map is along the length of the electrode. (b) Micro-optical density at $632.8 \mathrm{~nm}$ for several SWNT samples.

The measured optical transparencies for a few samples are shown in Figure 4.9b. Some of the samples, notably S17 and S4, show uniform coverage of SWNTs. The remaining samples show varying degrees of inhomogeneity. Sample S7 seems to contain a single region with a high density of material while the remaining areas are covered homogeneously. Samples S9 and S12 are particularly inhomogeneous. The trend in the optical density in sample S9, within the highlighted region, shows a hotspot-like spraying pattern, where the spray is thickest in the centre and decreases in thickness radially. Such a pattern can arise when the spray cone is too narrow, or when the sample is not at the centre of the spray cone. The spatial average and standard deviation of the optical transparency for the samples shown in Figure $4.9 \mathrm{~b}$ are tabulated in Table 4.1. This data is accompanied by the resistance measured at $300 \mathrm{~K}$ within the cryostat for three of the five samples. This data does not show the expected correlation between resistance and average optical transparency. In fact, the trend appears random. A possible connection between spatial homogeneity and VRH conduction is discussed in Section 4.4.5. 


\begin{tabular}{|c|c|c|c|}
\hline Sample & Resistance $(\Omega)$ & Sample & Resistance $(\Omega)$ \\
\hline \hline S1 & 42000 & S6Anneal & 13000 \\
\hline S2 & 303000 & S10 & 125000 \\
\hline S2Anneal & 83000 & S11 & 39000 \\
\hline S3 & 39000 & S12 & 18000 \\
\hline S3Anneal & 23000 & S13 & 44000 \\
\hline S4 & 16000 & S14 & 27000 \\
\hline S5 & 286000 & S16 & 14000 \\
\hline S6 & 22000 & S17 & 8000 \\
\hline \hline
\end{tabular}

Table 4.2: Table of tested samples (fabricated on sapphire) and their average resistance at 300 $\mathrm{K}$ (measured within the cryostat)

\begin{tabular}{|c|c|c|c|c|c|}
\hline Sample & Resistance $(\Omega)$ & Sample & Resistance $(\Omega)$ & Sample & Resistance $(\Omega)$ \\
\hline \hline G1 & 28000 & G2 & 73000 & G3 & 13000 \\
\hline \hline
\end{tabular}

Table 4.3: Table of tested samples (fabricated on glass) and their average resistance at $300 \mathrm{~K}$ (measured within the cryostat)

\subsection{Conduction mechanisms}

This section explores the prevalent mechanisms of conduction in our sprayed SWNT networks. Charge transport in pristine HiPCO SWNTs can be described by three parallel mechanisms (Eq. 3.6). The three mechanisms are prevalent in different temperature ranges. The metallic 'shunt' term describes the small residual conductance due to percolating metallic pathways within a network of non-metallic conducting pathways. At very low temperatures (below $\sim 4$ $\mathrm{K})$ the non-metallic pathways freeze out and only this small contribution from metallic tubes remains. Between $4 \mathrm{~K}$ and $220 \mathrm{~K}$, the VRH term recovers and dominates the conductance until the activation term turns on around $220 \mathrm{~K}$. As motivated in Chapter 3.4, our interest lies in identifying correlations between the macroscopic resistance and the parameters of the charge transport model: the tunnelling barrier size $\left(T_{0}\right)$, conductance pre-factor $\left(G_{0}\right)$ and activation energy $\left(E_{\text {act }}\right)$.

In order to isolate the prevalent conduction mechanisms in our SWNT networks, we measured the temperature dependence of the resistance for sixteen SWNT networks (sprayed on single side polished sapphire) using the PPMS (Chapter 2.4.2). The resistance of these samples at $300 \mathrm{~K}$ is given in Table 4.2. Three samples were also fabricated on glass coverslips. The resistance of these samples are given in Table 4.3 .

It should be noted that resistances above $10 \mathrm{M} \Omega$ were automatically disregarded by the PPMS electronics. Data was collected in one of two modes: (i) $300 \mathrm{~K}$ to $400 \mathrm{~K}$; $400 \mathrm{~K}$ to 2 $\mathrm{K}$ and $2 \mathrm{~K}$ to $400 \mathrm{~K}$ or, (ii) $400 \mathrm{~K}$ to $2 \mathrm{~K}$ and $2 \mathrm{~K}$ to $400 \mathrm{~K}$. Due to instrumental limitations on the highest measurable resistance $(7 \mathrm{M} \Omega$ ) data could only be collected above $10 \mathrm{~K}$ (for the lowest resistance samples). 
Data was analysed in context of the two main models presented in Chapter 4.4. However, conductance traces arising from the FIT and VRH mechanisms are qualitatively similar (Chapter 2.3.3 especially when the measured temperature range does not extend below $10 \mathrm{~K}$. Since any detailed analysis of the conductance data requires that only one mechanism dominate, we first present our preliminary analysis of deducing the dominant mechanism. We argue that $\mathrm{VRH}$ is the most appropriate mechanism for our samples.

The VRH formula (Eq. 2.25) can be reduced to change the conductance from non-linear to linear by plotting $\ln (\mathrm{G})$ as a function of $T^{\frac{-1}{d+1}}$. This transformation allows for more robust fitting with linear regression. The FIT formula, however, is difficult to reduce and any data that follows FIT can only be fit with non-linear regression. In order to identify if FIT or VRH is the dominant mechanism, we fit one representative dataset (sample S4) with Eq. 2.24, and linearised the data according to the VRH model as mentioned earlier. This analysis is shown in Figure 4.10. Two conclusions can be made from this figure: (i) FIT only fits the high temperature data to $\sim 200 \mathrm{~K}$, and (ii) The VRH model (2D VRH) appears to dominate across the full temperature range.

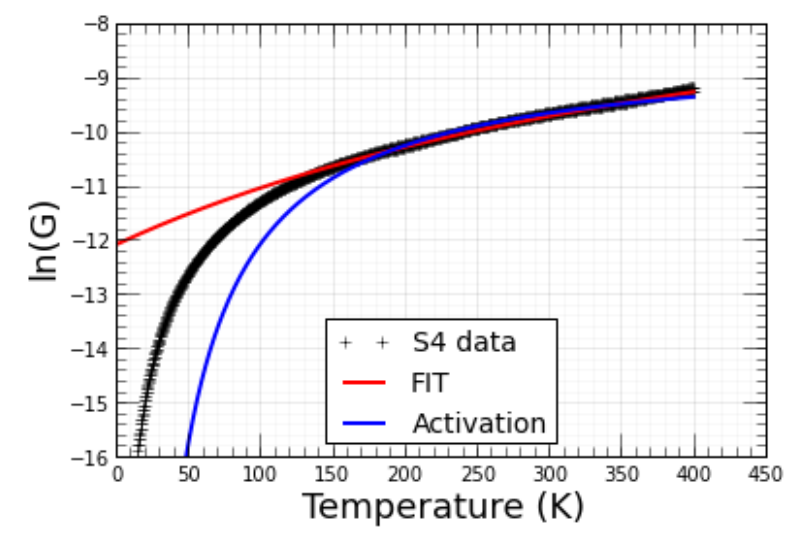

(a) FIT

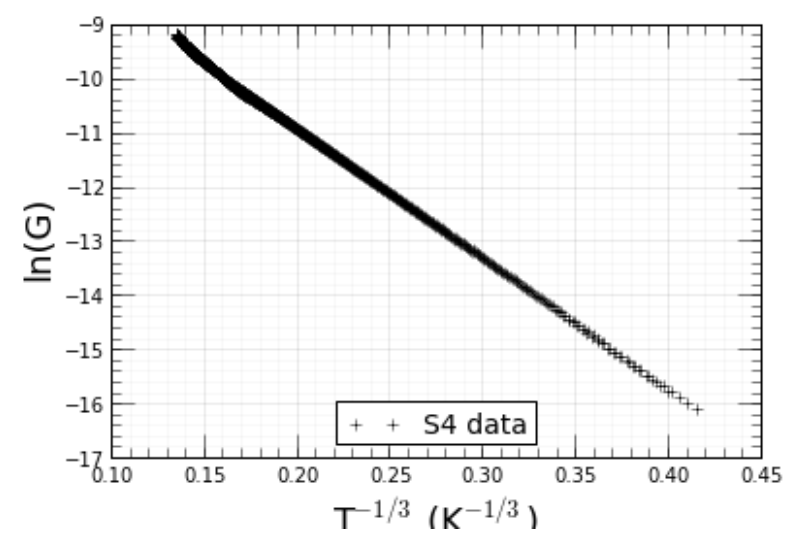

(b) VRH

Figure 4.10: Choosing the VRH dimensionality based on the extent of data that is well fitted.

The first point that FIT only fits the high temperature data could be interpreted as a basis for a model that incorporates FIT and a second mechanism that will play a bigger role at low temperatures. We can demonstrate that this scenario is unlikely by comparing the FIT fit with a fit of pure thermal activation. In Figure 4.10a, we can see that both FIT and thermal activation models cover the same temperature range. This shows that the high temperature limit of thermal activation is the part that actually fits the data. The critical part of the FIT model, that describes the effect of charge fluctuations, does not follow the data at all. Thus, we can conclude that the FIT model does not adequately model the conductance trace of sample S4. The VRH model, however, does follow the conductance curve for a large fraction of the measured temperature range (Figure 4.10b). In the following section, we detail how the con- 
ductance data is fitted with a composite model that is dominated by VRH and supplemented by thermal activation at high temperatures.

Thus, preliminary analysis of all the datasets showed that VRH was the primary conduction mechanism. The majority of the datasets also required the activation term to describe the conductance between $220 \mathrm{~K}$ and $400 \mathrm{~K}$. Since the measured temperature range only goes to $10 \mathrm{~K}$, the small contribution from pure metallic pathways (seen only below $4 \mathrm{~K}$ ) could not be determined. Eq. 3.6 now becomes:

$$
G=G_{0} \exp \quad-\frac{T_{0}}{T}{ }^{\frac{1}{d+1}^{\#}+G_{1} \exp \frac{-E_{a}}{T}}
$$

\subsubsection{Determining VRH dimensionality}

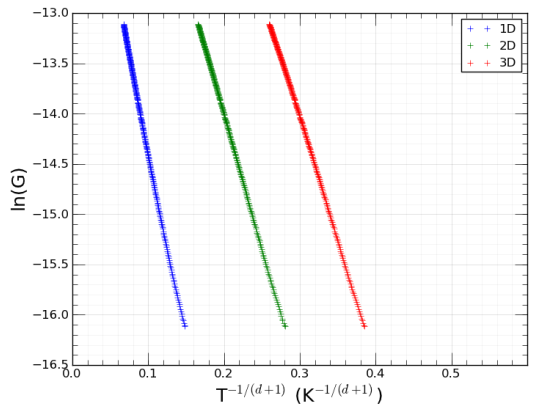

(a) Reduced data for $\mathrm{S} 2$

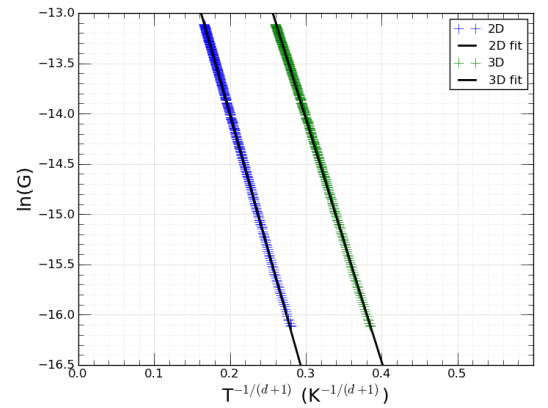

(b) S2 reduced data and linear best fit

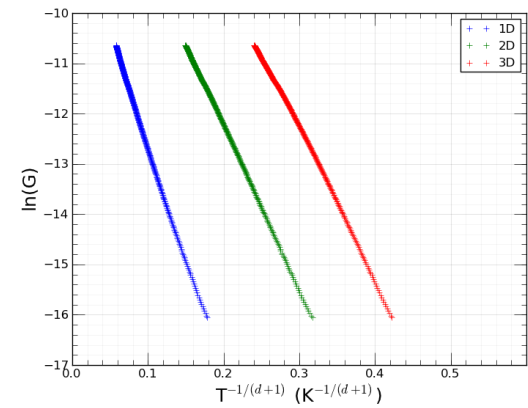

(c) Reduced data for S1

Figure 4.11: Choosing the VRH dimensionality by linearising the observed data.

The dimension of the hopping mechanism is an important parameter and goes some way to visualising the conduction process within disordered materials. However, determining the dimensionality of the conducting elements is not a clear process. There are several features that allow for choosing one dimensionality over another. The most useful feature is the linearity of the reduced data. Here, the experimental data is plotted with the log of conductance $(\ln (\mathrm{G}))$ on the vertical axis against $(1 / \mathrm{T})^{1 / d+1}$ as shown in Figure 4.11a. From this plot, 1D VRH can be easily eliminated but the differences between the $2 \mathrm{D}$ and $3 \mathrm{D}$ reductions are more subtle. A linear best-fit is plotted over the two ambiguous datasets to help discern which dimensionality is more linear (Figure 4.11b). A very close examination of the linear fit shows that the 2D VRH data reduction is more linear than the 3D VRH data. In this particular sample, the dimensionality is hard to pick out from the reduced data. In other cases, the appropriate dimensionality is much easier to pick out (Figure 4.11c) and there is no need for close inspection along with the linear best fit lines. 
The VRH formula shown in Eq 2.25 has three parameters: $d, T_{0}$ and $G_{0}$. It is worth noting that we have chosen to constrain $d$ rather than making it a fitting parameter. As discussed in Chapter 3.4, we want to track $T_{0}$ as a function of network resistance. Since $T_{0}$ is inherently linked to the dimensionality (Eq 2.26), we cannot compare $T_{0}$ values from fits with different $d$ values.

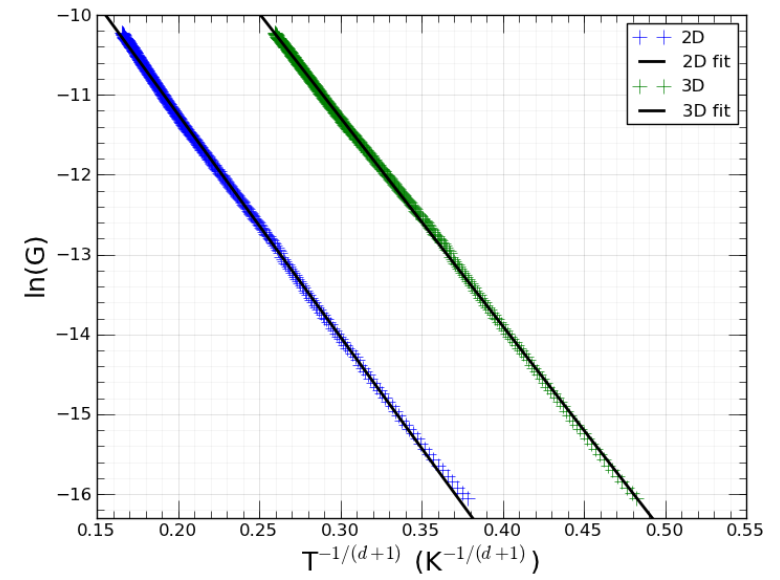

(a) S12 reduced data and linear best fit
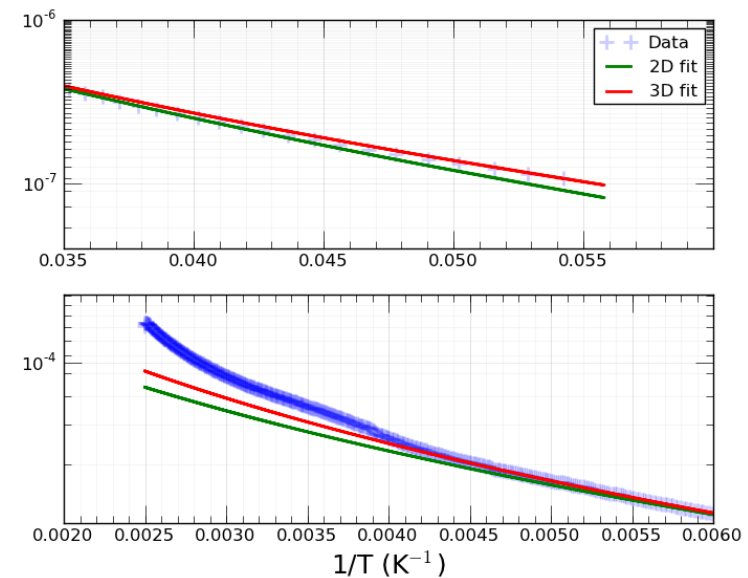

(b) S12 data fitted with 2D and 3D VRH

Figure 4.12: Choosing the VRH dimensionality based on the extent of data that is well fitted.

When the linear best fit shows that both the reduced datasets are equally good, choosing the most appropriate dimensionality becomes harder (Figure 4.12a). In such cases, the choice depends on the temperature range that is best fitted. Figure $4.12 \mathrm{~b}$ shows the low (top plot) and high (bottom plot) temperature extremes for this sample. The $2 \mathrm{D} \mathrm{VRH}$ fit is seen to veer away from the data sooner than the 3D VRH fit. Hence, the latter is chosen for the final fits.
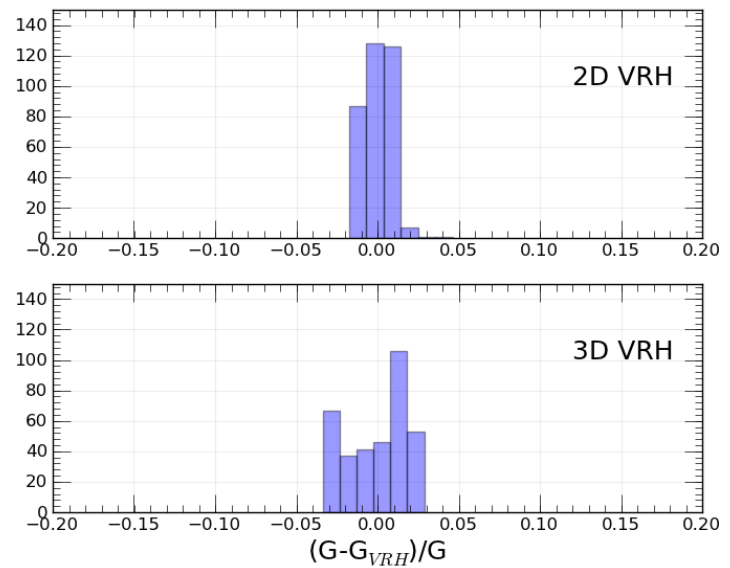

(a) Errors between data and fit for S2
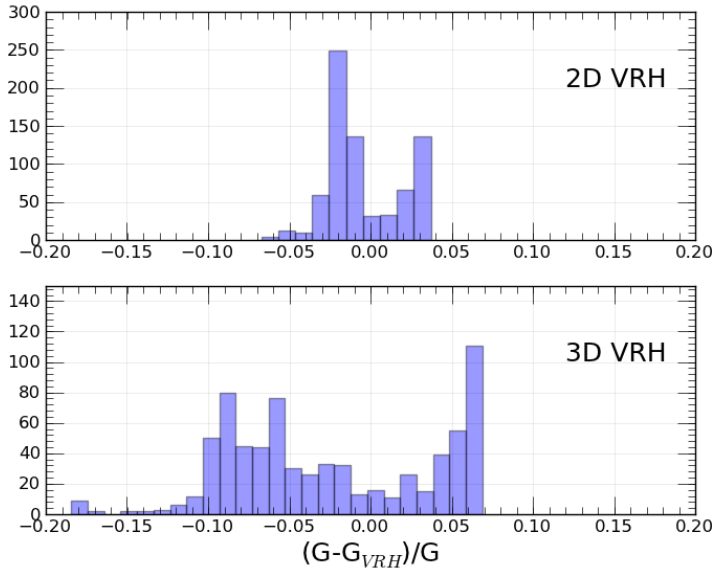

(b) Errors between data and fit for S1

Figure 4.13: Choosing the VRH dimensionality based on the magnitude of normalised error between the model and the data over the temperature range the model operates in. 
The two methods detailed above can be misinterpreted since they are based on visually isolating the trends/deviations. Instead of this approach, one can look at the residuals from the $2 \mathrm{D}$ and $3 \mathrm{D}$ VRH fits to the data and plot them either as a function of temperature or, as a histogram. In the first method, the sample $\mathrm{S} 2$ was shown to follow 2D VRH since the linear best fit identified a smaller non-linearity in the reduced data compared to the 3D VRH model. When the normalised errors between the model and data are plotted as a histogram, it can be seen that both models describe the data well and the 2D VRH fit is only marginally better than the 3D VRH fit (Figure 4.13a). For other samples, the fit by one model is considerably better than the other as seen for sample S1 in Figure 4.13b. This method of using the error distribution is robust and less subjective than the visual methods.

Our use of the error (residual) distribution width to determine the quality of the VRH fit can be considered unorthodox. Typical analyses of goodness of fit use the $\mathrm{R}^{2}$ value. Values fall in the range $[0,1]$ with 'good' fits having values as close to 1 as possible. We have not used $\mathrm{R}^{2}$ because it is not suitable for subtle comparisons. For example, the difference between $\mathrm{R}^{2}$ values of 0.55 and 0.9 are considerable. Not so the difference between 0.997 and 0.993 . In the earlier iterations of fitting, our goal was to compare the quality of the VRH fit across temperature. For this, we used the residual plotted as a function of temperature to find regions of good and poor fit. Thus, the use of the residual distribution continued further down the analysis.

The question of dimensionality is an important one but so is the temperature range that the model fits best. In the samples studied so far, the VRH model was found to fit well for the data below $\sim 220 \mathrm{~K}$. In some cases, the VRH model could fit the entire temperature range though this was quite rare. Isolating the VRH temperature range turns out to be an easier task compared to the temperature range fit by the activation model. Of the several routes attempted for fitting a dual model contribution to the data, the following route worked the best and is methodical enough to be followed for a large set of samples with different contributions from the activation and VRH conduction mechanisms.

First, the conductance data is fitted to the appropriate VRH model for the most suitable temperature range (typically all data below $220 \mathrm{~K}$ ). It is of note that only the cooling data was fitted for most samples as the heating data typically displayed local irregularities (Section 4.4.4). Then, the VRH model component is subtracted from the full dataset. This residual conductance is then plotted on log scale against temperature. The temperature region that is most linear is then fit with the activation model. The entire conductance dataset can be fit accurately with each model describing a clear region of data. The advantage of this method vs. fitting the full data to Eq. 4.2 is the quality of the fit. Fitting all the parameters in Eq. 4.2 gives what appears to be a good fit but closer inspection reveals that the VRH fit deviates away from the data in the low temperature region - a region where the model should fit the data accurately. Furthermore, the clear separation in the temperature regime for the two models - 
VRH for low temperature and activation for high temperature - is indicative that a separate fitting is the best approach.

\subsubsection{Physical origin of activation conduction}

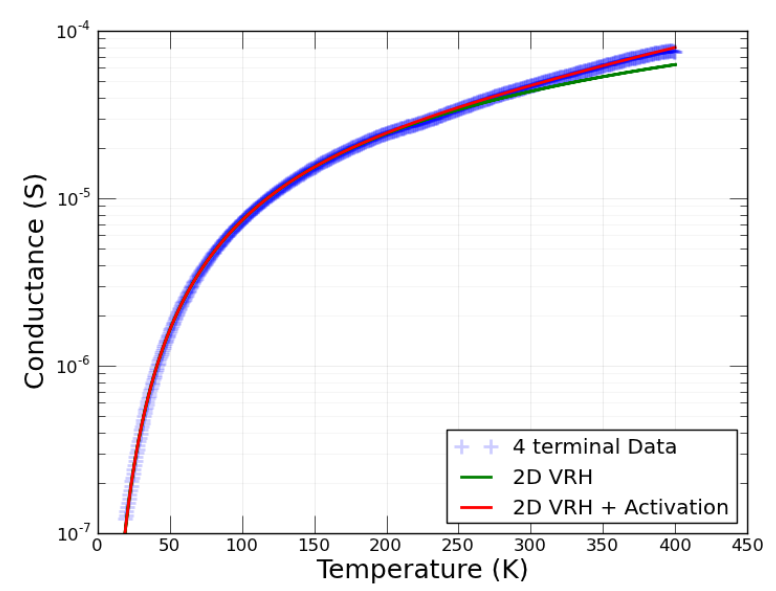

(a) Full temperature range

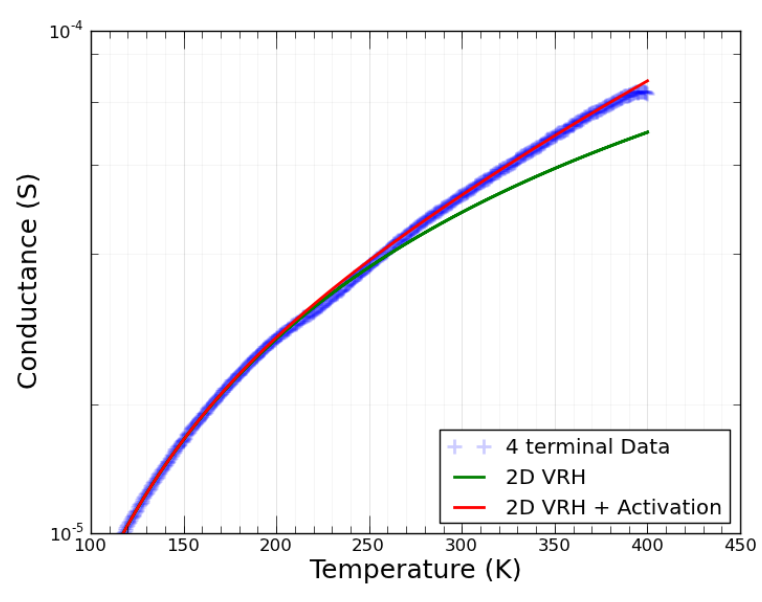

(b) Zoomed to high temperature data

Figure 4.14: Four terminal resistance data with 2D VRH and VRH + activation fits.

Most of the studied samples require the high temperature activation term to fully describe the temperature dependence of the resistance. In our earlier study [52], this term was ascribed to the activation of carriers over M-S junctions. The fitted activation energy of $220 \mathrm{meV}$ from our data agreed with experimental values of Schottky-like barriers at individual, crossed nanotube junctions $(250 \mathrm{meV}$ ) 90]. The presence of both this activation mechanism and the low temperature metallic percolation was attributed to a clear physical change: network densification after solvent removal. SEM images of remnant surfactant in the unannealed network [82] and Raman studies of the G-band linewidth provided additional evidence of increased physical contact between the nanotubes after solvent removal by heating. Here, a four terminal measurement of a pristine HiPCO film confirms our suggestion that the activation process occurs within the film rather than the nanotube/metal contact interface. The data and fits to this four terminal measurement are shown in Figures 4.14a and 4.14b. The fitted activation energy of $170 \mathrm{meV}$ is close to the activation energy from our earlier study. We can also show that the activation process is unrelated to atmospheric doping and is intrinsic to pristine HiPCO nanotubes.

An unannealed sample (prepared by wet spraying) was annealed in-situ within the PPMS sample chamber to avoid atmospheric doping. The effects of solvent removal on the sample resistance were tracked from 300 - $400 \mathrm{~K}$. Figure $4.15 \mathrm{a}$ shows the rapid decrease in resistance for temperatures above $20{ }^{\circ} \mathrm{C}$. The resistance stabilises around $90{ }^{\circ} \mathrm{C}$. Once all traces of solvent are removed without any atmospheric doping, the resistance data can be described by a combination of $3 \mathrm{D} \mathrm{VRH}$ and activation. Figure 4.15b shows cooling and heating data after 
in-situ annealing. The 3D VRH can be associated with the physical morphology of samples prepared by wet-spraying. These samples do not demonstrate uniformity in thickness due to the clumping of SWNTs after deposition (also see Chapter 6.3). Unlike in dry-spraying, wet solution on an unheated substrate can move around creating a 'clumpy' morphology. As described in Section 4.4.3, there is some correlation with inhomogeneous networks and 3D VRH.

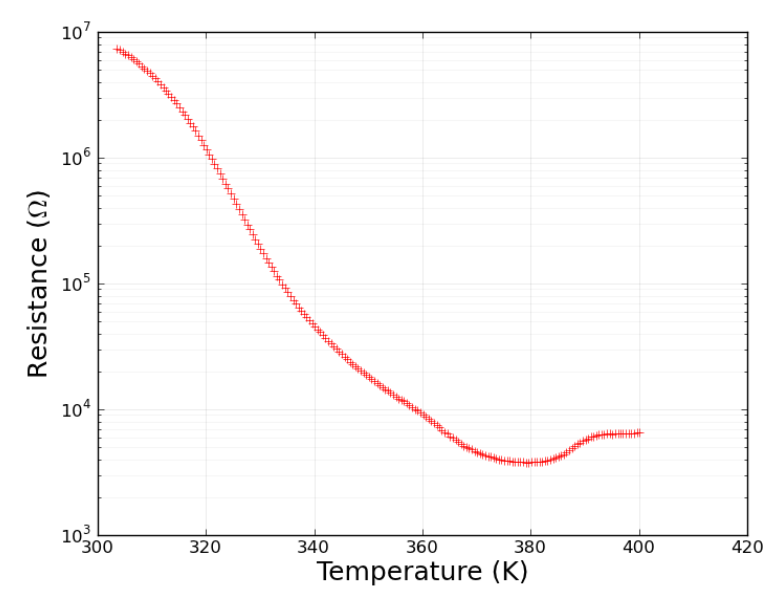

(a) In-situ solvent removal

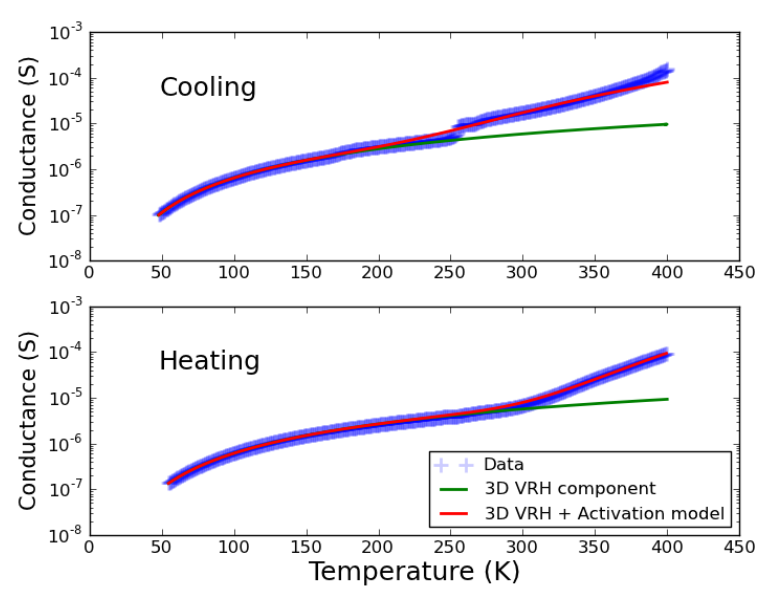

(b) Conduction mechanisms

Figure 4.15: In-situ annealing. (a) Change in resistance as remining solvent is removed. (b) Conduction mechanisms within the annealed sample.

Though both heating and cooling data are described by 3D VRH and activation, a clear hysteresis separates the two curves. The dataset collected immediately after annealing shows a jump in conductance around $250 \mathrm{~K}$. This discontinuity bears some resemblance to dopant desorption processes. Other types of local irregularities, seemingly unrelated to dopant desorption, feature in other resistance curves. In Section 4.4.4, the different types of local irregularities are examined in greater detail.

\subsubsection{Effect of clumps}

As mentioned in our notes on the fabrication method, the SWNT solution was not centrifuged in this iteration of experiments (Section 4.2.1). As a result, the solution retained large clumps of SWNTs - invisible to the naked eye but capable of clogging the nozzle during spray deposition. The build-up of solution behind the blockage eventually forces the spraying to resume but the clumps are sprayed across the sample. In Section 4.3, the distribution of these clumps were detected using the micro-Raman system. In some samples, the clumps appear to heavily dominate the morphology and in others, they are barely present. With this small set of over-lapping conductivity and micro-morphology data, we suggest that VRH dimensionality is linked to sample morphology. 
In Chapter 2.3.2, the $T_{0}$ parameter was described in terms of the localisation length in addition to the density of localised states about the fermi level. With the right estimates of either the density of localised states or the coherence length, changes to $T_{0}$ can be discussed in a qualitative manner. Changes in the VRH dimension, however, can only be discussed qualitatively. Here, we have linked the VRH dimension to morphology. 3D VRH dominates in a sample with large distribution of clumps while 2D VRH dominates in samples with a small distribution of clumps. The intricacies of hopping conduction in these two different systems is best explained visually (Figure 4.16).

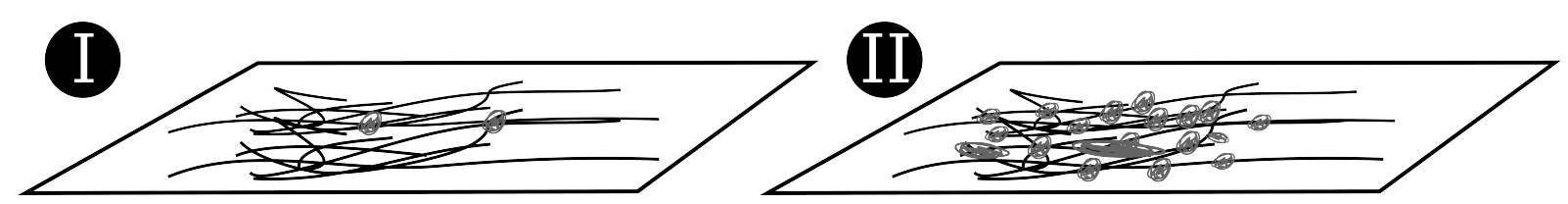

Figure 4.16: Cartoon schematic of two extreme types of SWNT networks: small distribution of clumps (I) and a large distribution of clumps (II).

In scenario I (with a large distribution of clumps), hopping proceeds in three-dimensional space due to the large density of nanotubes and thus, available localised states. This rationale of a high density of localised states also results in a smaller localisation length. In scenario II (small distribution of clumps), the lower density of available localised states restricts hopping within a 'plane'. From this, we can extrapolate that localisation lengths are longer in these 'clump-free' networks.

In summary, we speculate that clumps affect the localisation length since they affect the density of localised states available for hopping. However, the scenarios posed in Figure 4.16 is a simplistic one. The clear-cut distinction of the schematic is a representation of the extreme cases. In reality, the range of possible scenarios are likely to be between scenarios I and II: samples with a significant distribution of clumps as well as clump-free regions. Perhaps an interesting future experiment would be a study of the density of clumps as a function of the VRH dimension, which is now a free fitting parameter.

\subsubsection{Local irregularities in temperature-dependent resistance}

SWNTs exposed to air contain some degree of adsorbed atmospheric dopants (Chapter 3.3) e.g. oxygen and possibly water vapour. The influence of these adsorbates on the network resistance are much larger when the SWNT network is comprised mainly of semiconducting tubes. The major adsorbant, oxygen, can be desorbed by heating for a prolonged period of time [50] or by UV exposure [91]. The desorption of adsorbed oxygen, or other species that increase carrier density, increases the resistance of the SWNT network: between 30 [91] - 50\% [50] of the initial resistance. This sensitivity to adsorbed dopants is greatest when the network resistance is dom- 
inated by non-metallic conducting paths. Such paths typically dominate in (i) thin networks made from a mixed sample (1:2 ratio of M:S nanotubes) or (ii) in a thick film made from a selectively enriched sample of semiconducting tubes. Increasing the metallicity of the conducting paths by either adding more tubes or increasing the ratio of metallic tubes, sees a decrease in the resistance sensitivity to adsorbants [50]. The features related to dopant presence and subsequent desorption are seen in the resistance traces from our SWNT networks. However, we also note the presence of an additional effect where heating decreases the resistance. We tentatively attribute this to desorption of molecular species that reduce carrier densities when present, i.e. they disadvantageously dope the SWNTs.

Prior to measuring the temperature dependence of the resistance, most samples were heated in an ambient furnace to either (i) 30 minutes at $50{ }^{\circ} \mathrm{C}$ or (ii) 10 minutes at $100{ }^{\circ} \mathrm{C}$. This step ensured that the majority of the adsorbed oxygen was desorbed from the network in addition to curing the GE varnish that secured the samples on the measurement puck. In some cases, this process also cured the silver epoxy (used to repair broken connections to the sample). Sample resistance was measured in ambient before $\left(R_{i}\right)$ and after $\left(R_{f}\right)$ the heating step. The blue crosses in Figure 4.18a shows that there is a consistent $40 \%$ increase in resistance after heating. The trend indicates that mild heating (30 minutes at $50^{\circ}$ and 10 minutes at $100^{\circ}$ ) dedope the SWNT network. Despite this heating step, two dopant desorption-related features were seen in the resistance data: a downturn in resistance at high temperatures and, a bump at $250 \mathrm{~K}$.

The metallic-like upturn in resistance is shown in Figure 4.17a. It is only seen in the first cycle of cooling. The upturn corresponds to a de-doping of the network [50] and thus, only occurs once. Another feature, a bump at $\sim 250 \mathrm{~K}$, is also seen regularly in our samples. This feature is tentatively assigned to desorption of adsorbed water [50]. In most cases, the bump occurs in the heating curves. The nature of the bump, however, varies from mild (bottom plot in Figure 4.17b) to significant (top plot in Figure 4.17b). From this, we can surmise that despite the resistance change after heating, the SWNT networks have not been adequately desorbed of dopants. Thus, the heating cycles during measurement induce a series of desorption processes that result in hysteresis of the resistance trace. In fact, if the SWNT network is measured without any curing, the resistance traces show significant hysteresis even into the second cooling cycle (Figure 4.18b).

At present, the only correlation found between samples showing different degrees of hysteresis is the dimensionality of VRH. All of the five samples described by 3D VRH display significantly greater hysteresis between the heating and cooling curves compared to the samples described by 2D VRH. Though the correlation is interesting, the underlying reasons for a link between VRH dimensionality and the resistance hysteresis still remains unclear. It should be noted that this regular discontinuity in the data presented some problems in fitting the activation term. Hence, the conduction mechanism analyses were carried out for the cooling 
dataset alone.

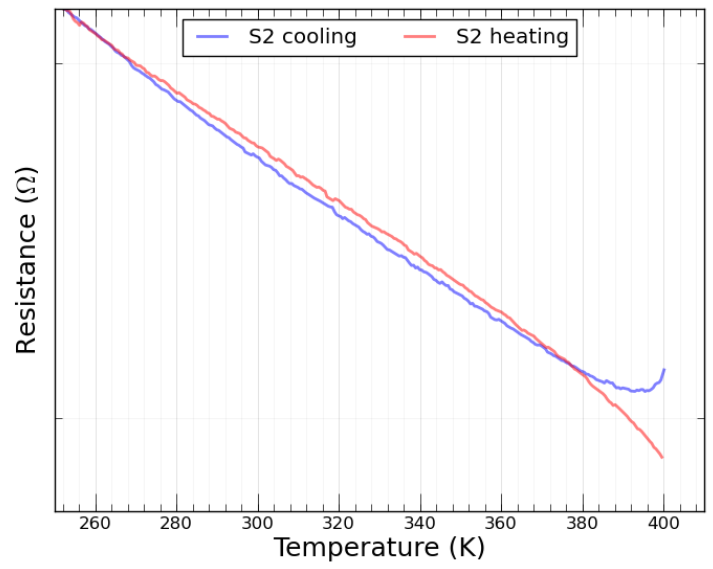

(a) Metallic-like decrease in resistance
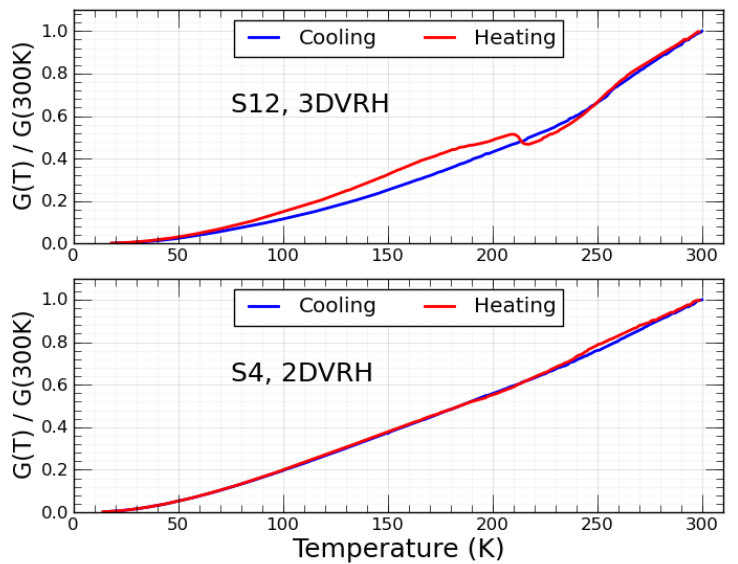

(b) Dopant desorption around $200-250 \mathrm{~K}$

Figure 4.17: Dopant desorption effects. (a) Metallic-like downturn that only appears in the first cooling. (b) Hysteresis in the resistance during heating cooling cycles for two types of samples.

However, two other types of heating have a completely different effect on the sample resistance. The first is internal heating (within the cryostat) to $400 \mathrm{~K}$ within the PPMS and the second, external heating to $320^{\circ} \mathrm{C}$. For both types of heating, the resistance changes by a similar magnitude but in the opposite direction to the desorption data (blue crosses in Figure 4.18a. In the case of internal heating to $400 \mathrm{~K}$, the samples that have already been through the furnace 'desorption' process change perceptibly. This feature is unexpected given previous work which showed that the resistance stabilises after desorption. The favourable change in resistance suggests the desorption of adsorbants that disadvantageously dope the network, or a physical change in the network that improves conduction e.g. defect annealing [92].

The suggestion of improvements to the network conductivity was tested by measuring a set of samples before and after external annealing in a tube furnace (ambient atmosphere) at 320 ${ }^{\circ} \mathrm{C}$. The resistance of the samples was measured within the cryostat at $300 \mathrm{~K}$ (red crosses in Figure 4.18a). This set of samples were also cured in the ambient furnace prior to measurement but their resistance is still consistently lower than before the high temperature anneal. Whatever the mechanism behind this change, the barrier size of the network remains the same. Figure 4.18c shows the normalised conductance curves for the samples before and after annealing. Though the annealed samples have a lower resistance, the conductance trace is unchanged. The average barrier size for this set of curves is $19 \times 10^{3} \mathrm{~K} \pm 2800 \mathrm{~K}$. Only the $G_{0}$ values are consistently larger after annealing, indicating a larger number of conducting pathways [91]. The reduction in resistance after external annealing favours a mechanism that causes a physical change in the network, rather than the desorption of disadvantageous dopants. Annealing has been shown to improve conductivities in SWNT networks by annealing defects [92]. However, 


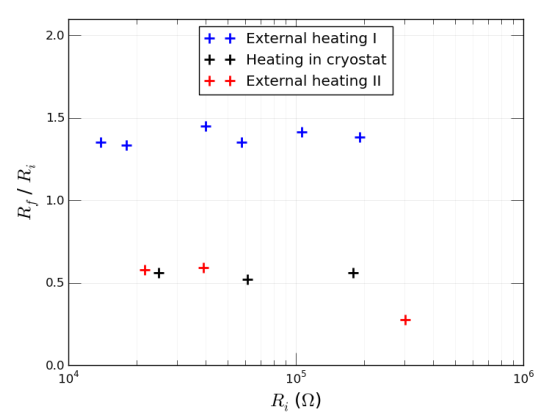

(a) Different effects of heating

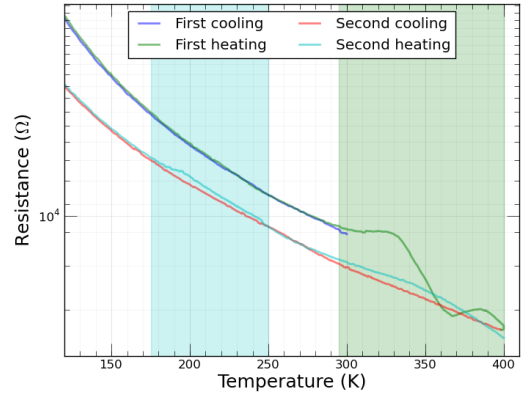

(b) Hysteresis in resistance data

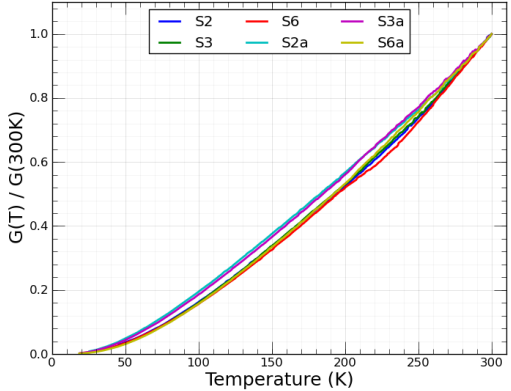

(c) Conductance curves for external heating I

Figure 4.18: (a) Changes to the sample resistance after different types of heating. (b) Hysteresis in resistance data for an uncured sample that hasnt been de-doped to any extent prior to measurement. (c) Normalised conductance plots for dry-sprayed samples before and after annealing (labelled with 'a') at $320{ }^{\circ} \mathrm{C}$.

further study is required to support this mechanism over the desorption effects.

In summary, we have presented dopant desorption and an as-yet unknown mechanism that affects the sample resistance after heating. The sensitivity of the network resistance to these mechanisms suggests that conducting paths are dominated by semiconducting tubes. Since the changes are independent of resistance, the entire sample range - from $300 \mathrm{k} \Omega$ to $8 \mathrm{k} \Omega$ - can be associated with non-metallic conducting pathways.

\subsubsection{Summary statistics}

This final section brings together emergent trends from the VRH + activation model fits to all the samples. The premise for this body of work revolved around finding a correlation between barrier sizes and the sample resistance. From VRH fits to the data, more than $70 \%$ of the samples made on sapphire followed 2D VRH. The remainder could be fitted with 3D VRH. The prevalent 2D VRH is likely due to the M:S ratio. A study by Yanagi et al. [49] found dominant 2D VRH for mixed samples with a majority of semiconducting tubes. When the majority electronic type of the mixed sample became metallic, the VRH dimension changed to 3D. The HiPCO process produces nanotubes with a 1:2 electronic type ratio and we have made no changes to this ratio by discarding nanotubes that are not selectively bound from solution. Therefore, the underlying mechanism for 3D VRH can be attributed to a different mechanism. We speculate that this mechanism is spatial homogeneity.

VRH conduction analysis for three of the samples (S4,S12,S17), whose spatial homogeneity was characterised in Section 4.3, showed that 2D VRH dominates in the homogeneous samples, S4 and S7, while 3D VRH dominates in the spatially inhomogeneous sample, S12. Similarly, all samples fabricated on glass coverslips could only be described by $3 \mathrm{D}$ VRH. In the case of glass, 
the rough substrate morphology causes considerable variation in the $z$ dimension of the sprayed network compared to the same network on a planar substrate like sapphire. This correlation is only a suggestion but it does present an intuitive picture of the hopping process in three dimensions: when large clumps are present in the sample or, when a thin network is sprayed on an undulating substrate.

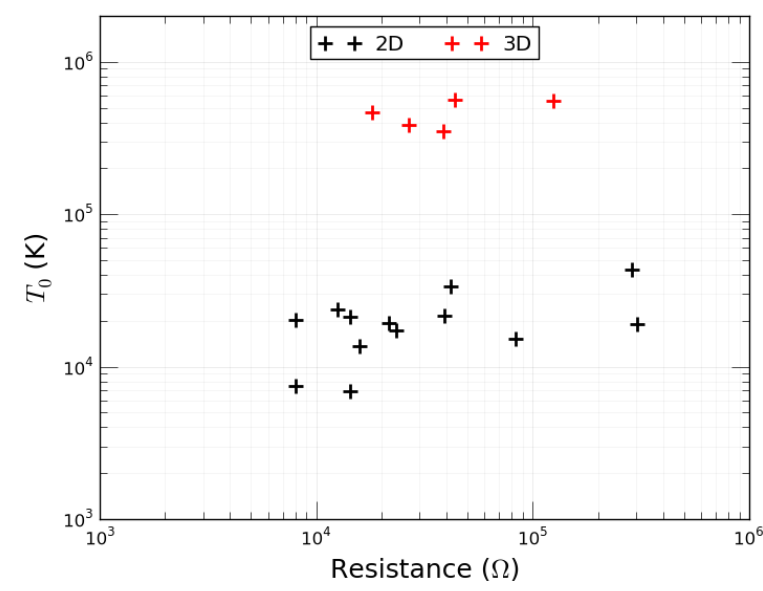

(a) $T_{0}$ vs. sample resistance

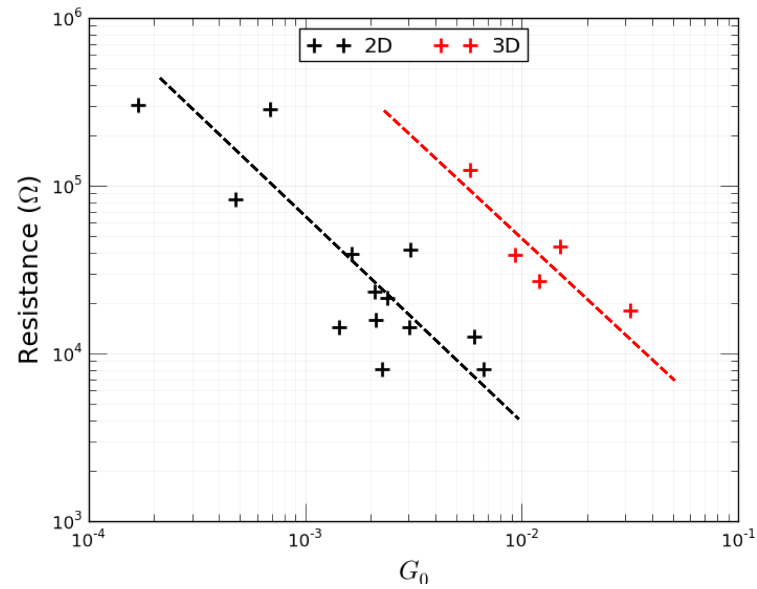

(b) $G_{0}$ vs. sample resistance

Figure 4.19: (a) Sample resistance vs. barrier size $\left(T_{0}\right)$. (b) $G_{0}$ vs. sample resistance (at 300 $\mathrm{K})$. The dashed lines are a visual aid.

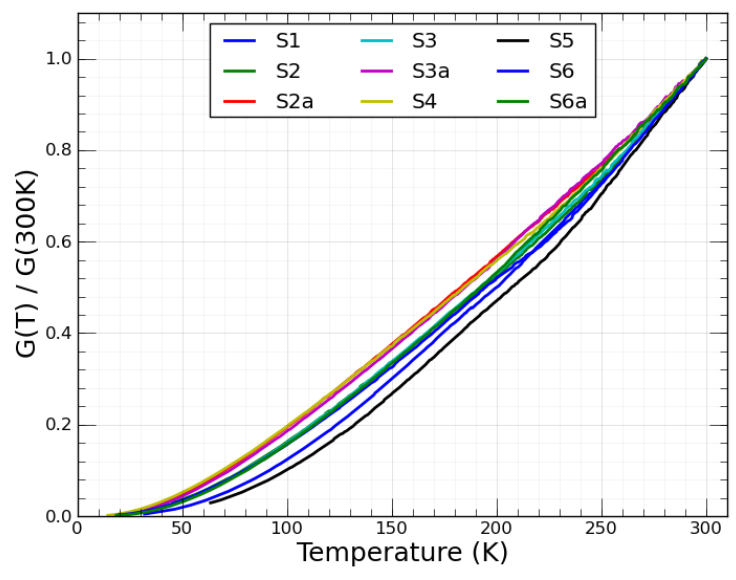

(a) Normalised conductance

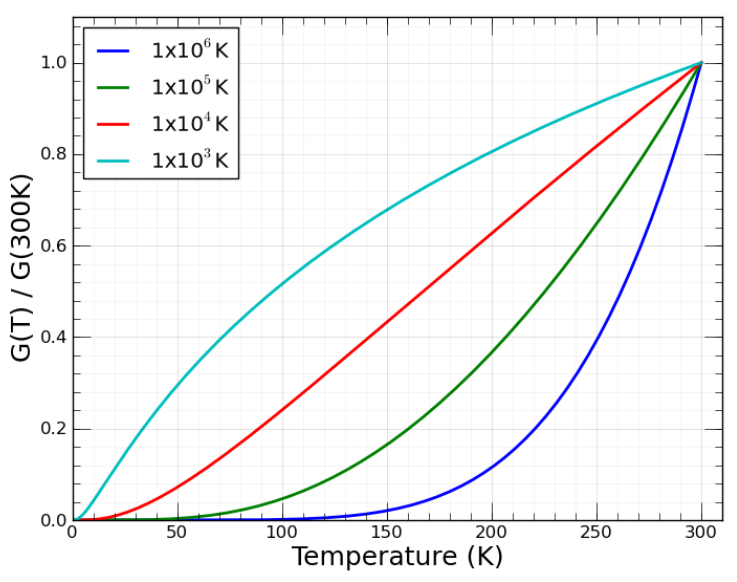

(b) Effect of $T_{0}$ on $G(T)$

Figure 4.20: (a) Normalised conductance curves after dopant desorption. All the samples plotted here demonstrate 2D VRH. (b) Simulated 2D VRH curves with variable barrier sizes.

The $T_{0}$ parameter from both $2 \mathrm{D}$ and $3 \mathrm{D}$ VRH fits is plotted against the sample resistance at $300 \mathrm{~K}$ in Figure 4.19a. There appears to be no correlation between resistance and barrier size. Despite the increase in conducting pathways as the resistance decreases, the intrinsic nature of the conduction processes continues to be dominated by non-metallic pathways. The 
non-metallicity of the SWNT networks for sample resistances between $300 \mathrm{k} \Omega$ and $8 \mathrm{k} \Omega$ is attested by both the sensitivity of the resistance to atmospheric adsorbants. The unchanging microscopic conduction processes can also be seen in the consistent shape of the normalised conductance curves in Figure 4.20a. Without the distracting local irregularities of desorption processes seen in the log plots, the linear plot shows that the normalised curves collapse on top of each other. A simulation of the conductance curve (2D VRH) for different barrier sizes is plotted in Figure 4.20b. The change in barrier size clearly affects the shape of the resistance trace. That this change does not occur in our samples is apparent from the tight cluster of the traces in Figure 4.20a. Thus, the only parameter that changes with resistance is $G_{0}$, which has a clear linear correlation with decreasing resistance (Figure 4.19b).

This null result suggests a limiting barrier size for HiPCO networks in general or, for the non-metallic conduction regime in HiPCO networks. The samples examined so far seem to be within this regime despite having resistances as low as $8 \mathrm{k} \Omega$. Thus, the changeover from non-metallic to metallic pathways lies at a lower resistance.

The activation process for samples demonstrating 3D VRH or $2 \mathrm{D} \mathrm{VRH}$ is essentially the same. The average activation energy is $160 \pm 20 \mathrm{meV}$ and $160 \pm 60 \mathrm{meV}$ for $3 \mathrm{D}$ and $2 \mathrm{D} \mathrm{VRH}$ respectively. The consistency in the fitted activation energy confirms our findings that activation is an intrinsic process within the films that only depends on crossed nanotube junctions.

\subsection{Conclusions}

This chapter presented results of uniting the macroscopic and microscopic pictures of conduction. We were able to controllably fabricate samples with resistances between $300 \mathrm{k} \Omega$ and 8 $\mathrm{k} \Omega$. The conduction mechanisms for this range of samples were subsequently identified from the temperature-dependent resistance. The majority of the samples could be described by 2D VRH alone to $\sim 220 \mathrm{~K}$. The remaining samples followed 3D VRH to the same maximum temperature. Beyond $220 \mathrm{~K}$, an activation process, with an activation energy of $\sim 160 \mathrm{meV}$, had to be taken in account. Four terminal measurements showed that this activation process occurs within the film; most likely at crossed junctions between metallic and semiconducting nanotubes (Schottky-like junctions). The fitted values of the activation energy corresponds with the expected value of Schottky-likes barriers in HiPCO nanotubes seen in Figure 3.7 ; $100 \mathrm{meV}$; and individual metal/semiconductor crossed junctions: 190-290 meV [90]. In our previous study, the origin of this mechanism was attributed to decreased spatial barriers after solvent removal [52]. The observation of VRH and activation in samples annealed only to 110 ${ }^{\circ} \mathrm{C}$ (dry-sprayed samples) supports our premise of pristine HiPCO films.

The two possible types of hopping mechanism (2D or 3D VRH) showed no dependence 
on sample resistance. The only correlation between hopping dimensionality is the spatial homogeneity of the sprayed sample. From the parallel study of microscale variations in sample homogeneity, samples with a spatially uniform network followed 2D VRH and samples with a non-uniform spatial coverage of SWNTs followed 3D VRH. At this stage, this link is suggestive as the study of spatial homogeneity was only performed for a small subset of samples. Nonetheless, it presents an interesting prospect for future research: the relationship between morphology and dimensionality in pristine SWNT networks.

For both $2 \mathrm{D}$ and $3 \mathrm{D} \mathrm{VRH}$, the barrier size, $T_{0}$, remained unchanged with resistance. The value of $T_{0}$ for $2 \mathrm{D}$ VRH fluctuated unpredictably about $20,000 \mathrm{~K}$ by $\pm 9400 \mathrm{~K}$. Thus, this study is the first to demonstrate a limiting junction barrier size in HiPCO networks or, the non-metallic regime of HiPCO networks. Across the full range of samples, the samples showed a strong sensitivity to atmospheric adsorbants, indicating that despite increasing contribution from metallic pathways with decreasing resistance, the non-metallic pathways still dominate. That this non-metallic regime may have a limiting barrier size raises further questions about the relationship between barrier size and resistance. The original expectation of reducing barrier sizes with increasing contributions from metallic pathways is no longer the only possibility. Perhaps, the changeover from non-metallic to metallic conduction is characterised by an abrupt change in limiting barrier size? Or perhaps, the barrier size stays the same regardless of the regime? A simple extension of this study (using sample resistances lower than $8 \mathrm{k} \Omega$ ) is required to resolve these questions in addition to the ones posed in Chapter 3.4 . 


\section{Chapter 5}

\section{Raman spectroscopy of SWNT networks: literature review, methodology and characterisation}

\subsection{Introduction}

Non-destructive characterisation of the component nanotubes carried out using Raman spectroscopy can reveal considerable information about the nanotube population within the sample. All single-walled carbon nanotubes exhibit a characteristic mode called the radial breathing mode $(\mathrm{RBM})$ whose vibrational frequency is inversely proportional to the nanotube diameter. Unique identification of the nanotube from its RBM is possible with a certain amount of information namely, the laser energy, the diameter distribution for the batch of samples and, the density of nanotubes under the probing laser spot. Most Raman studies performed on individual nanotubes or small bundles can unambiguously identify the $(n, m)$ indices from the RBM. Following this assignment, the studies can examine in detail the effect of different environments - both physical and chemical - from the the RBM lineshape. Typically, this type of study is achieved by monitoring the RBM while the laser energy is varied. The Raman signal from nanotubes is a resonance process due to the sharp electronic transitions or Van Hove singularities, intrinsic to $1 \mathrm{D}$ materials (Chapter 3.2). The discrete energies of these electronic transitions - which are typically between 1 and $3 \mathrm{eV}$ - mean that the intensity of the Raman signal can be tuned as a function of laser energy. Using this strategy, the behaviour of the underlying electronic transitions has been studied as a function of temperature and local environment e.g. solubilised in surfactant and on a substrate [76]. The entire nanotube population in a given sample [93] can also be studied using this strategy for insight on enrichment by chirality, diameter or electronic type.

Studies on the effects of local environment, temperature, and even doping on individual nanotubes is of both fundamental and technological importance. Knowledge and understanding of the individual componentry can predict some of the properties of macroscale devices 
fabricated from individual nanotubes such as random networks. However, the properties of the macroscopic devices themselves must be probed independently in order to establish whether the principles identified for individual tubes hold for the ensemble. Establishing such scaling laws is not a trivial matter. The RBM spectrum gets increasingly complex as the number of nanotube neighbours increase. The spectrum also contains many more peaks due to changes in the electronic transitions of the sampled nanotubes. Increasing the population of resonant nanotubes makes it correspondingly difficult for unambiguous assignments of the $(n, m)$ index. Furthermore, the relative ratios of the different types of nanotubes is not a constant in random networks since they have a high degree of local heterogeneity in nanotube density. As a result, the RBMs of SWNT networks are a relatively unstudied field.

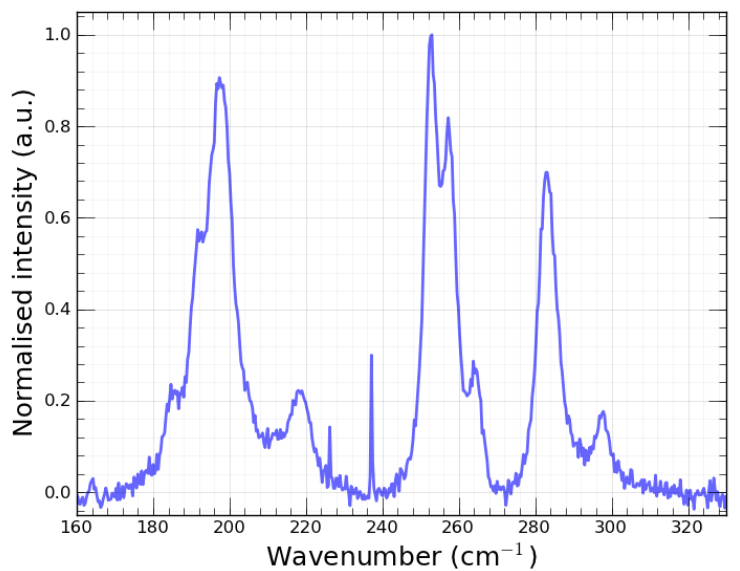

(a) Solution

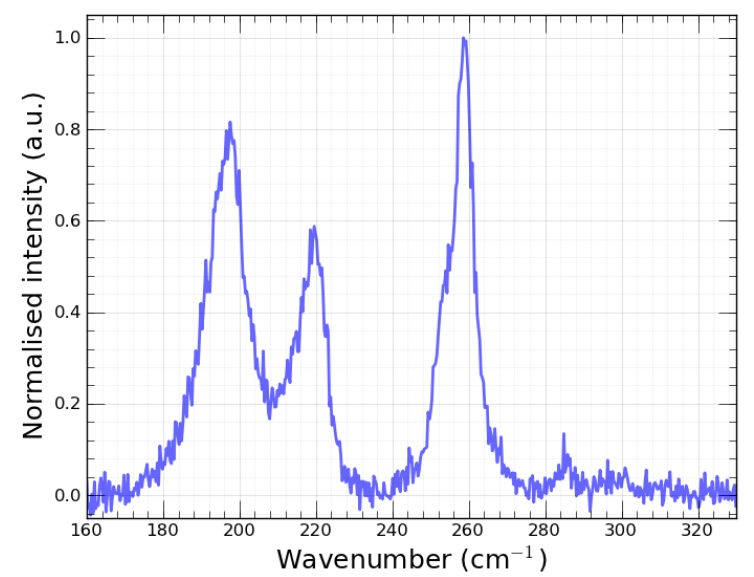

(b) Thin film

Figure 5.1: RBM spectrum from SWNTs in solution (a) and a thin film (b).

One of the main hurdles that must be overcome is spectral deconvolution. Figure 5.1 shows the RBM spectrum for HiPCO tubes solubilised in a stable dispersion and from a region on a thin film that has been heated to remove all traces of solvent. The use of simple peak finding techniques to deconvolute the spectrum shown in Figure $5.1 \mathrm{~b}$ would result in component peaks that are physically insignificant since the peak shoulders are very poorly resolved. Thus for a quantitative study of the influence of external parameters on the RBMs in thin films, we begin by outlining a rigorous methodology for deconvoluting complex RBM spectra; even those with few discernible spectral features. Accurate deconvolution allows the study of two important descriptors of the nanotube local environment: the resonance window and the peak lineshape (linewidth, intensity and position). These descriptors provide a holistic picture of the resonance condition, local temperature and bundling environment from which we can infer the effects of subtle treatments like surfactant removal, ambient and laser heating using an appropriate set of control samples.

In the following chapters, we showcase Raman spectroscopy as a diagnostic tool for thin films 
using two approaches. The first approach presents a high resolution deconvolution methodology that can accurately model the entire RBM spectrum. The second approach presents a spatial summary of a select few modes for any given sample. The accuracy and resolution of the first approach make it an ideal candidate to study properties of highly bundled tubes that are spatially invariant e.g. doping, temperature, or pressure. The deconvoluted RBMs can be tracked between samples or as a function of a tunable parameter giving a rich set of data that can be analysed by metallicity, diameter or chirality. The second approach provides a quick way of analysing the spatial variation within a sample by just deconvoluting one composite from the RBMs. It is useful for studying properties that could change within the heterogeneity of the sample or to provide an ensemble average of the chemical/physical environments. In this thesis, we use the high resolution method to study the temperature dependence of the RBMs from bundled nanotubes and the spatial method to track physical/chemical changes of the film fabrication process. The literature review, methodology and characterisation of the film fabrication process are covered in this chapter. The follwing chapter, Chapter 6, both presents the results of the high resolution temperature dependence study and compares it with a spatial study of local heating.

\subsection{The Raman spectrum of carbon nanotubes}

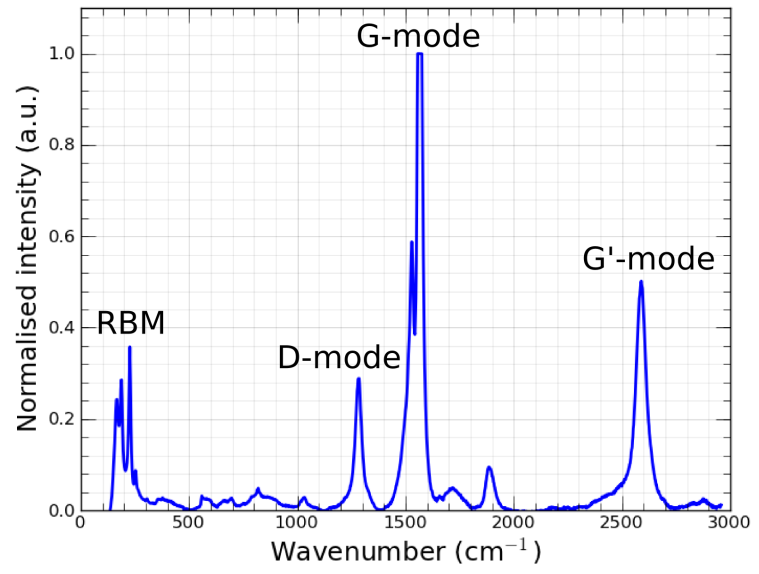

(a) Full Raman spectrum of SWNTs

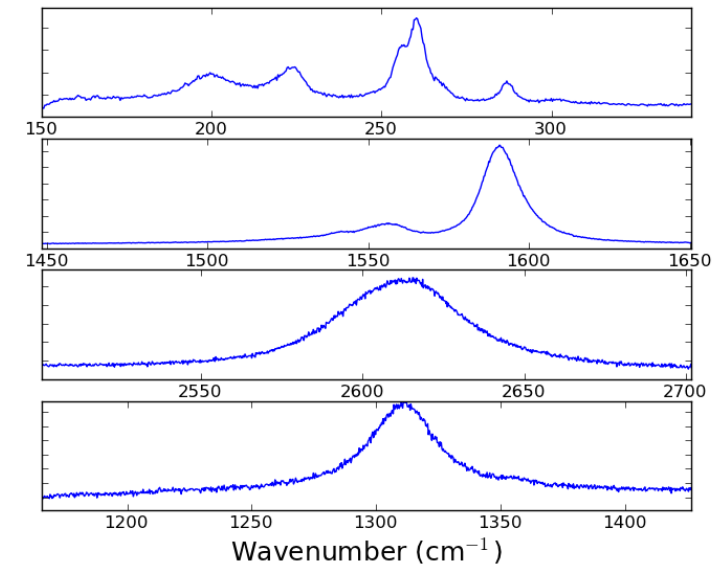

(b) The four main modes in detail

Figure 5.2: Raman spectrum of SWNTs.

The Raman spectrum of carbon nanotubes contains a mixture of modes inherited from from its parent: graphite as well as modes that are uniquely its own (Figure 5.2). The inherited modes include the G-mode ('graphite' mode), D-mode (a so-called 'defect' mode) and the G'-mode. The radial breathing modes or 'RBMs' on the other hand arise from quantum confinement of a single graphite sheet. The quantised dimensions of the nanotube also splits the single G-mode vibration into six components. 


\subsubsection{Kataura plot}

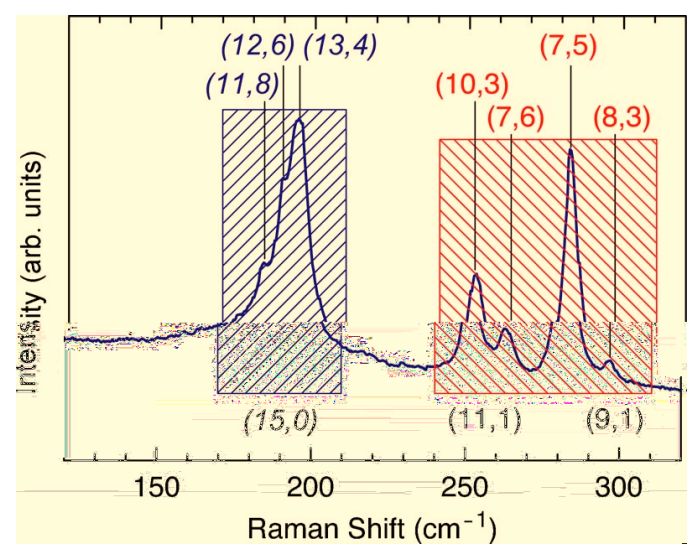

(a) RBM spectrum

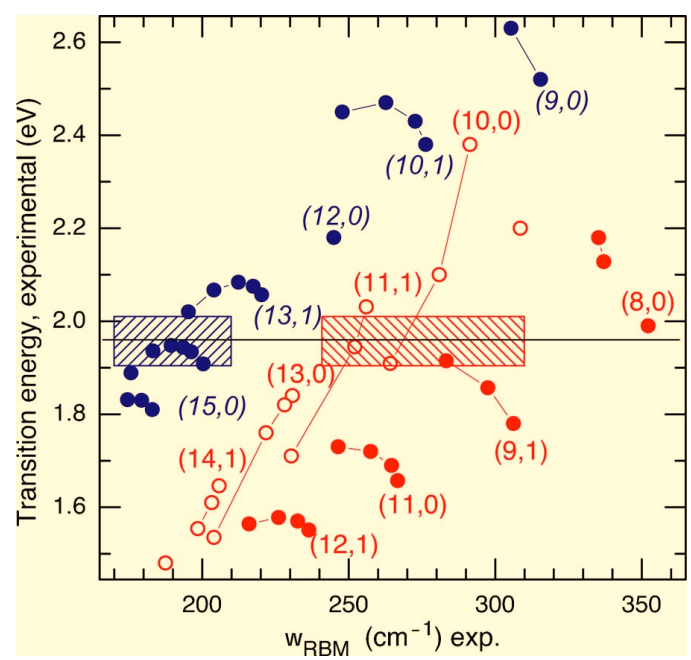

(b) Kataura plot

Figure 5.3: (a) RBM spectrum from SDS-solubilised HiPCO SWNTs. (b) Resonant nanotubes shown in a Kataura plot. Both plots taken from Maultzsch et al. 994.

The RBM vibration is aptly described by its name: the motion is a in/out (breath-like) movement in the radial direction. As a result, the frequency of vibration is strongly dependent on the size of the nanotube ring namely, the nanotube diameter. The relation that converts between the breathing mode frequency and the nanotube diameter $\left(d_{t}\right)$ is a simple linear equation: $\omega_{R B M}=A / d_{t}+B$. The parameters $A$ and $B$ have a range of values but it is agreed that the $B$ parameter is an 'environmental' parameter that changes depending on the sample, temperature, and medium. Recently, Araujo et al. [95] introduced a model that reduces the relation above to $\omega_{R B M} \overline{\overline{\mathrm{p}}} 227.0 / d_{t}$ and derived an expression for all other data in the literature to be: $\omega_{R B M}=227.0 / d_{t} \overline{1+(0.057) d_{t}^{2}}$. This global expression was derived using a simplified analogy of the nanotube and its environment as a coupled system comprised of two spring constants: one from the C-C bond strength and another from the interaction between the SWNT and its surroundings.

An early theoretical study by Kataura et al. [96] predicted the Van Hove transition energies $\left(E_{i i}\right)$ as a function of nanotube diameter. A plot of transition energies vs. $\omega_{R B M}$ or diameter is known as a Kataura plot and is the basis for identifying the $(n, m)$ indices from the Raman spectrum. While the theoretical model of the Kataura plot and subsequent refinements allow for fundamental understanding of the transition energies, they are not useful for experimental assignments as shown in Figure 5.3 for one main reason: nanotubes cannot be fabricated in a way that reproduces the full permutation of $(n, m)$ indices with equal probability of fabrication for a given nanotube. Different processes fabricate only a subset of possible $(n, m)$ indices and selectively enrich certain chiralities, diameters or electronic type. This process enrichment and 
any subsequent enrichment by solubilising the nanotubes in specific surfactants can be studied with an experimental Kataura plot where the SWNT sample is probed with a pseudo-continuum of laser energies. The experimental Kataura plot for HiPCO tubes (Figure 5.3b) is used as the basis for assignment in this chapter.

Figure 5.3 shows one example from literature [94] where the RBM spectrum of a solubilised sample of HiPCO tubes is matched with an experimental Kataura plot. The energetic dimensions of the Kataura plot is narrowed down to $E_{\text {laser }} \pm 0.05 \mathrm{eV}$ as shown by hatched boxes in Figure 5.3b. This energy window is referred to as the 'resonance window' and it describes the energetic spread of the $E_{i i}$ from the resonant nanotubes. In samples where the nanotubes exist as individuals or in small bundles, the resonance window is within tens of meV of the laser energy. As bundles increase in size, the resonance window not only broadens but also shifts in energy along the Kataura plot. The physical reason for this is the effect of the environment on $E_{i i}$ and is expanded upon in Section 5.2 .2 .

\subsubsection{Resonance effects}

The match between the sharp electronic transition energies and the probing laser energy is a resonance effect and gives rise to the strong Raman signal in SWNTs. Changes to the $E_{i i}$ affect the resonance condition which results in a subtle or drastic change in the RBM intensity. In this section, a background literature review and a simple model demonstrate how the degree of bundling and sample temperature influence the electronic transition energies and thus the resultant $\mathrm{RBM}$ spectrum.

\section{Bundling}

The strong van der Waals interaction between $s p^{2}$ carbon in graphite is also present in SWNTs. Raw nanotube powder resembles a fluffy flocculate and is comprised of large bundles of nanotubes. Bundling has been shown to be the cause of changes in the frequency and Raman intensity of the RBMs. Visual changes in the Raman spectrum - appearance and disappearance of various RBMs - can also be attributed to bundling since it causes the electronic transition energies to both redshift and broaden (Figure 5.4). The redshifts are typically of the order of tens of meV and found to be $\sim 70 \mathrm{meV}$ when compared to SWNTs solubilised in SDS [76].

\section{Temperature}

The redshift of the transition energy with temperature could be the result of three possible mechanisms: decrease in the overlap integral with temperature [97], electron-phonon coupling [98] and non-istropic pressure from the thermal expansion of the surrounding environment 


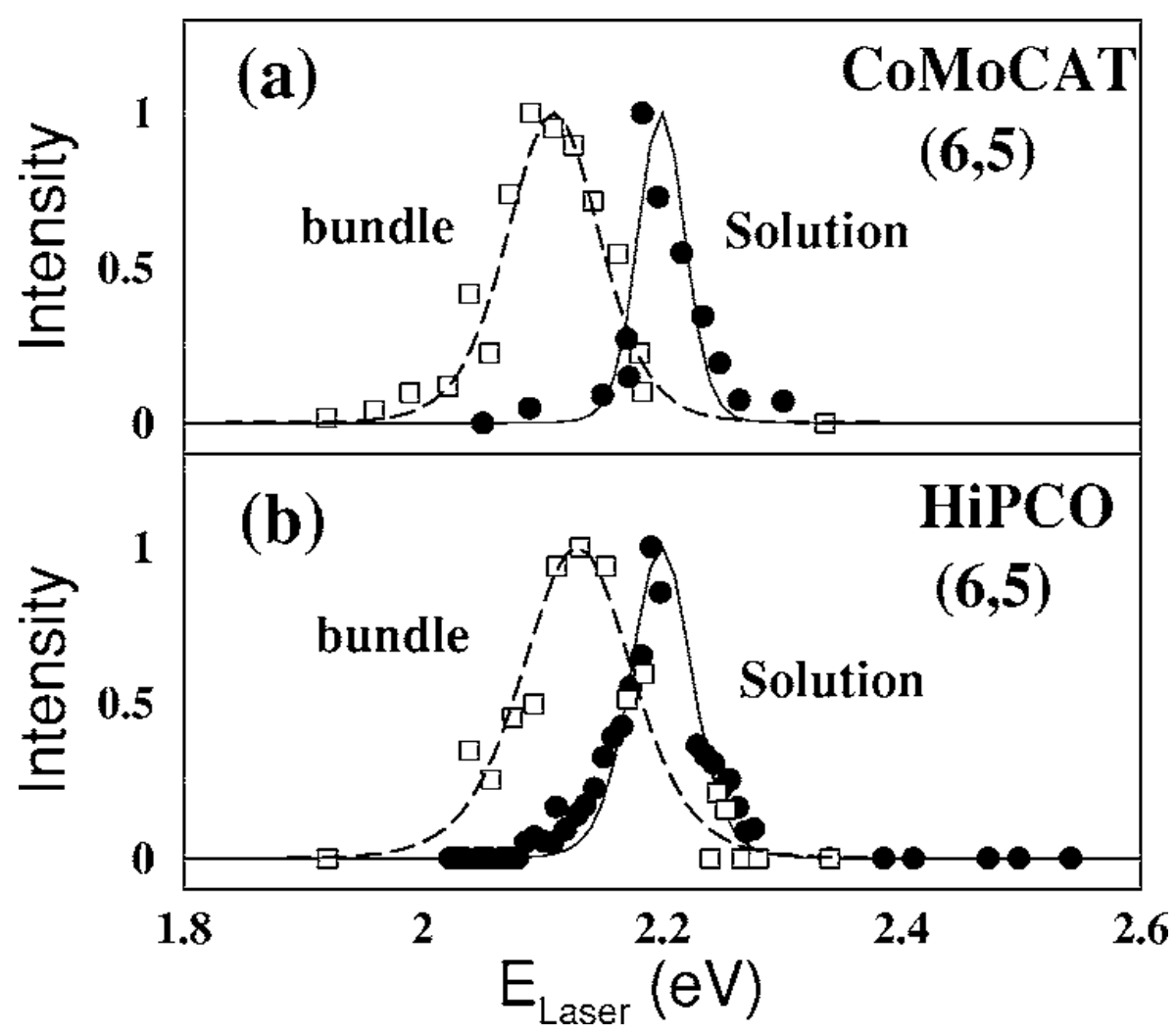

Figure 5.4: $E_{i i}$ of the $(6,5)$ SWNT from two different fabrication process in a bundle and in solution [93].

[98, 76]. In surfactant-coated or bundled nanotubes, the thermal expansion of the surrounding environment affects the transition energies of a given nanotube similar to a changing strain [99]. The transition energies are found to redshift with increasing strain with the exception of semiconducting nanotubes with $\nu=+2(\nu=((2 n+m) \bmod 3))$ where the $E_{i i}$ actually blueshifts with temperature [76] (unfilled circles in Figure 5.5). Eq. 5.1 gives a simple analytical form of $E_{i i}$ with a linewidth, $\gamma$, that can also be used to model the temperature dependence. In individual, suspended nanotubes, electron-phonon coupling is found to be the dominant mechanism for the redshifting and broadening of $E_{i i}[98$.

$$
E_{i i}=\frac{1}{E-E_{i i}+k_{B} T-\gamma} \quad \frac{1}{E-E_{i i}-k_{B} T-\gamma}{ }^{2}
$$

\section{Modelling RBM spectra}

Bundling causes a broadening of the resonance window by changing the transition energies such that they are red-shifted and broadened. Using this intrinsic change to the transition energies, Heller et al. [100] were able to qualitatively model RBM spectra from nanotubes in different bundling environments. We have modelled RBM spectra a little differently. The modelled spectrum in Figure 5.6a shows the expected RBM spectrum according to the experimental data 


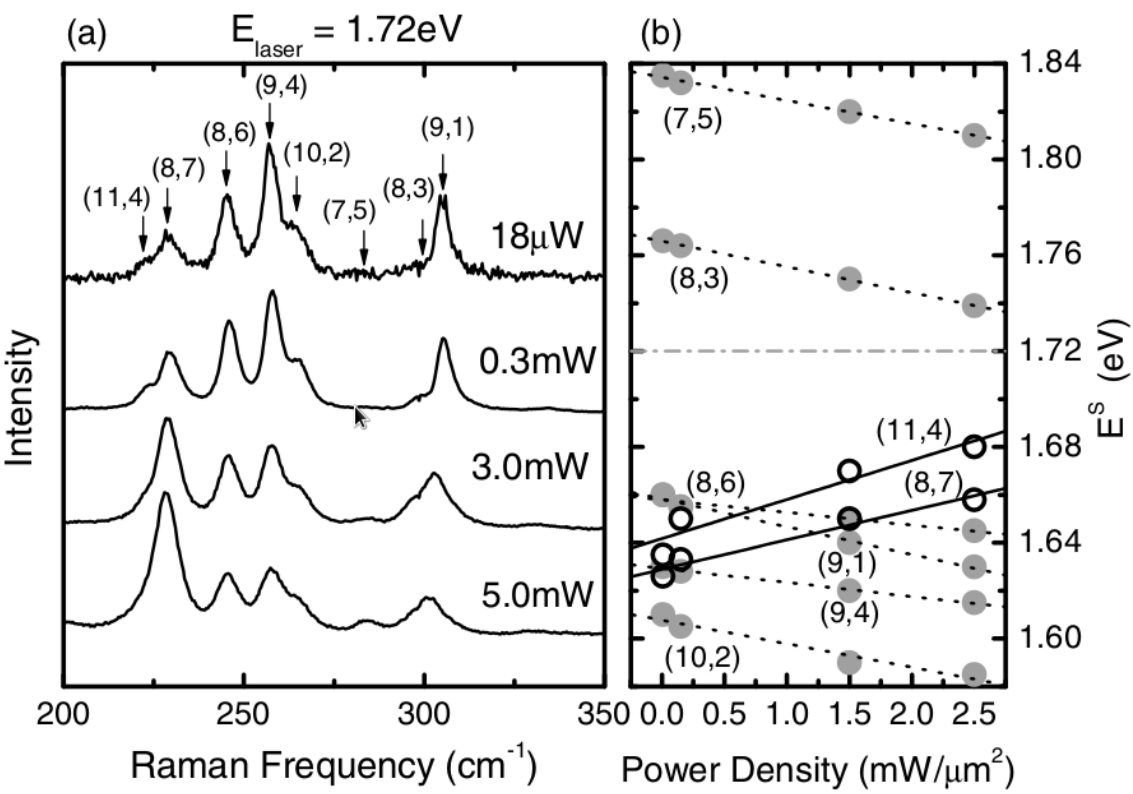

Figure 5.5: Local heating effects on the change in $E_{i i}$ in bundled HiPCO nanotubes [76].

for HiPCO tubes by Maultzsch et al [94]. The RBM intensity for each nanotube (Eq. 5.2) is given as the function of the diameter distribution $(P(d))$ [59], the intensity of the normalised resonance profile at $E_{\text {laser }}$, and the Raman cross-section $(\chi)$ [94. The qualitative agreement between both the solution spectra and the modelled spectrum is excellent but a discerning eye will find that several spectral features are not accurately modelled e.g. the missing $256 \mathrm{~cm}^{-1}$ peak in the SDS solution and more importantly, the increase in intensity of the composite at $200 \mathrm{~cm}^{-1}$.

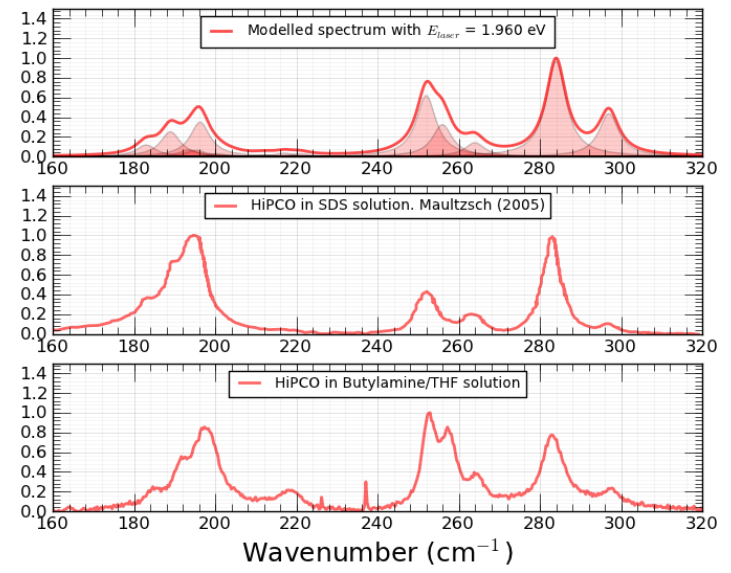

(a) Solution RBM spectra

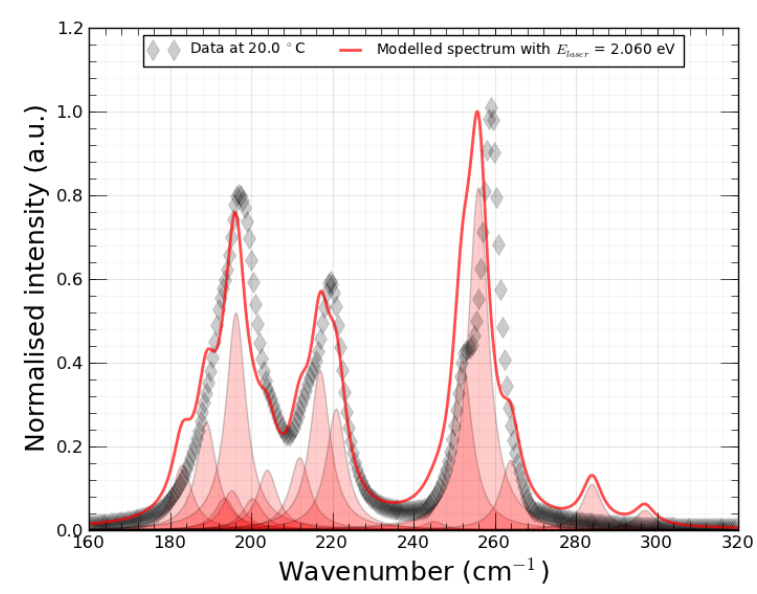

(b) Thin Film RBM spectrum

Figure 5.6: (a) Modelling RBMs according to a diameter distribution [100] and Raman crosssection [94]. (b). Modelling RBMs including population enrichment.

$$
I((n, m))=\chi \times P(d) \times \frac{1.0}{E_{l}-E_{i i}-i \gamma / 2}-{\frac{1.0}{E_{l}-\hbar \omega_{R B M}-E_{i i}-i \gamma / 2}}^{2}
$$


where $P(d)=1.0 /(\sigma \sqrt{4 \pi}) \exp \left(-(d-<d>)^{2} /\left(4 \sigma^{2}\right)\right)$ [100]. The values for $d, E_{i i}, \chi$ and $\omega_{R B M}$ - for tubes that can be uniquely identified after deconvolution - are obtained from Maultzsch et al. 994].

The inaccurate modelling in Figure 5.6a likely arises due to selective enrichment of certain nanotubes. This can arise at different stages of the fabrication process: from the synthesis of the SWNTs to the solubilisation to post-deposition treatments. In thin films, we can also see spatially inhomogeneous enrichment due to local population variation in bundled regions. Solution spectra are a little more representative of the sample population as the total Raman signal is accumulated from a large volume that is in a constant state of flux due to convection and photon pressure from the laser. With the addition of a term accounting for nanotube population enrichment, we can get closer to a represenation of real RBM spectra. Figure 5.6b shows one example of spectral modelling. The starting criterion for matching the model to the data was to expand the resonance window to include the $220 \mathrm{~cm}^{-1}$ composite. Following this, the laser energy was changed such that the modelled signal for this composite (which also happens to contain nanotubes with diameters close to the the mean diameter for HiPCO SWNTs) matched the observed data. The final touchups were adjustments to the relative population of individual tubes at: $252 \mathrm{~cm}^{-1}, 264 \mathrm{~cm}^{-1}, 283 \mathrm{~cm}^{-1}$ and $297 \mathrm{~cm}^{-1}$, in addition to an increase in the population of the large diameter metallic tubes clustered about $200 \mathrm{~cm}^{-1}$.

The qualitative representation of RBM spectra could impart some information about selective enrichment, bundling or temperature effects. However, it is worth noting that the enrichment of an entire family of tubes $\left(200 \mathrm{~cm}^{-1}\right)$ is not very accurate. From the same note of variability in enrichment across different areas of the sample in SWNT films, there could also be the complete disappearance of signal from an expected resonant nanotube. It is for this reason that modelling RBM spectra from a basis set of resonant nanotubes does not give a quantitative picture of the observed data. Furthermore, the broadening of spectral features $\left(E_{i i}\right.$ and linewidth) reduces the visual impact of hidden peaks within a larger composite i.e. composites lose well-defined shoulders. In order to address these problematic features of RBM spectra from bundled SWNTs, we describe a novel way to deconvolute spectra in the following section.

\subsection{Methodology: deconvoluting complicated Raman spec- tra}

The use of the second derivative to identify unresolved peaks in the spectrum is a well-known deconvolution technique in absorption spectroscopy while it is rarely seen in Raman spectroscopy. This is largely due to the fact that Raman spectroscopy probes vibrational modes at the zone center (very small dispersion in vibrational frequency) rather than a continuum of states as is 
the case with absorption spectroscopy. In many cases, even the convoluted spectra of the radial breathing mode region in roped samples can be fit reliably without considering the second derivative as the component peaks can be seen as distinct peak shoulders. However, the complexity of the RBM region increases considerably with higher density of material. Hence, any analysis of thick nanotube networks requires a reasonably robust fitting routine that can pick out RBM components not directly visible in the spectrum itself. We suggest one analysis route that uses the increased resolution afforded by higher order derivatives to deconvolute complicated RBM spectra where spectral features like peak shoulders are too subtle. The following sections detail the procedure of a deconvolution routine that provides stable and interactive analysis of RBM spectra. Variations of this routine could be used to fit large spectral sets by simply eliminating the interactive component or by modifying it slightly.

\subsubsection{Introduction to second derivatives}
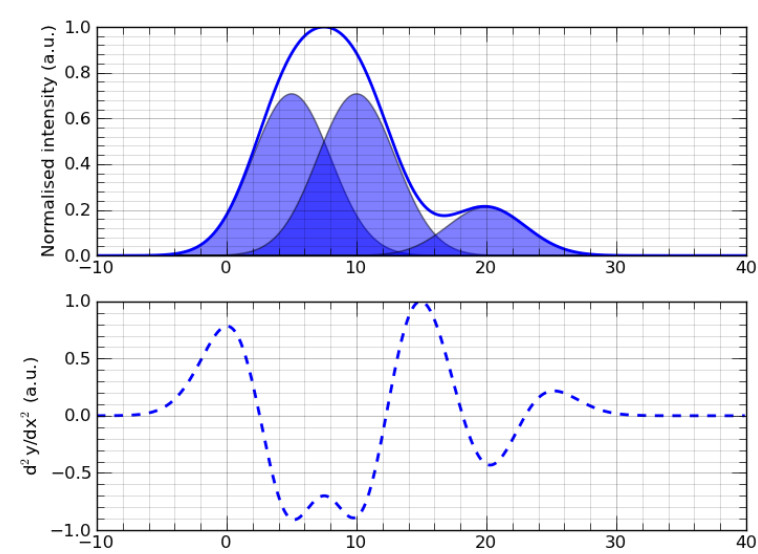

(a) Gaussian
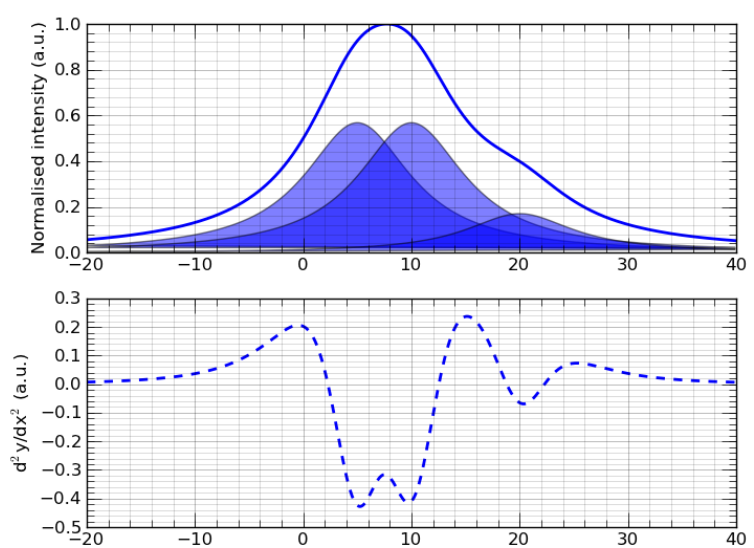

(b) Lorentzian

Figure 5.7: Convoluted spectra comprised of component gaussians (a) and lorentzians (b) with FWHM $(\Gamma)=6$.

Empirically, it can be shown that the second derivative can resolve two peaks provided their peak maxima are separated by at least $80 \%$ of the FWHM. At this limit, the composite spectrum looks like a single peak (top plots in Figure 5.7) while the second derivative clearly resolves two peaks. The second derivatives (bottom plots of Figure 5.7) of the gaussian and lorentzian were calculated analytically using the formulae given in Eq. 5.3 and Eq. 5.4 respectively. The constants $a, b$ and $c$ are the intensity, position and width respectively. For the gaussian, $c$ is proportional to the linewidth by the relation: $c=\Gamma /\left(2^{p} \overline{(2 \log 2))}\right.$ while $c$ is the half-linewidth for a lorentzian.

In their highly-detailed article, Mark and Workman [101] highlight several important parameters to be aware of when calculating higher order derivatives. They make a particular note 
of the wavelength resolution for the numerical evaluation of second derivatives. They illustrate that increasing the wavelength spacing leads to errors in the shape of calculated derivatives while increasing the derivative intensity. This trade-off, while interesting, does not impact the numerical calculation of second derivatives from Raman spectra due to the high wavelength resolution available using fine gratings (e.g. 1800 lines/mm). Raman spectra collected for this chapter have a resolution of $0.01 \mathrm{~nm}$ compared to the $1 \mathrm{nnm}$ resolution typical of absorption spectra. We have compared the second derivative spectra from the raw data compared with interpolated data and noticed little to no difference between the two indicating that the raw data is good enough for all analyses of the second derivative.

$$
\begin{gathered}
d^{2} y / d x^{2}=\left(-a / c^{2}\right) \exp -\frac{(x-b)}{\sqrt{2} c}{ }^{\#}+\left(-a / c^{2}\right)(x-b)^{2} \exp -\frac{(x-b)}{\sqrt{2} c}^{2} \\
d^{2} y / d x^{2}=\left(2 a / c^{2}\right) \quad \frac{\left((2 x-2 b)^{2}\left(1 /\left(c^{2}\right)\right)-\left(1+((x-b) / c)^{2}\right)\right.}{\left(1+((x-b) / c)^{2}\right)^{3}}
\end{gathered}
$$

\subsubsection{Fitting spectra}

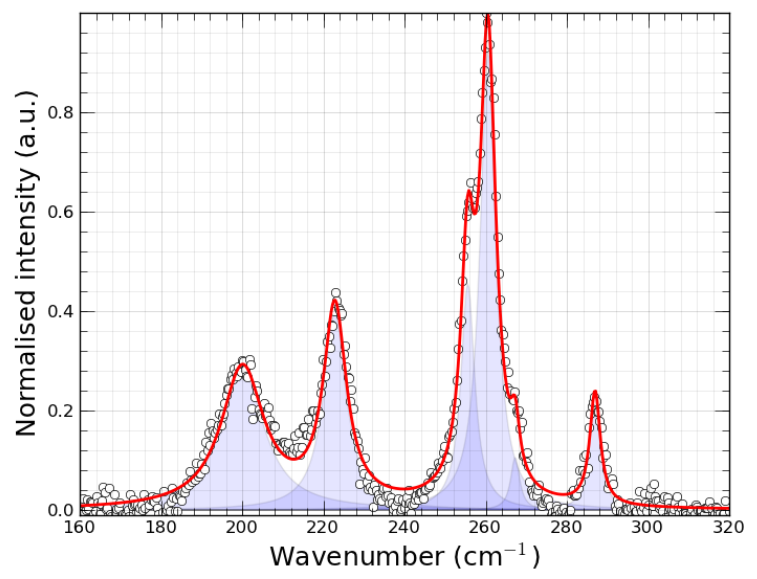

(a) Software peak detection

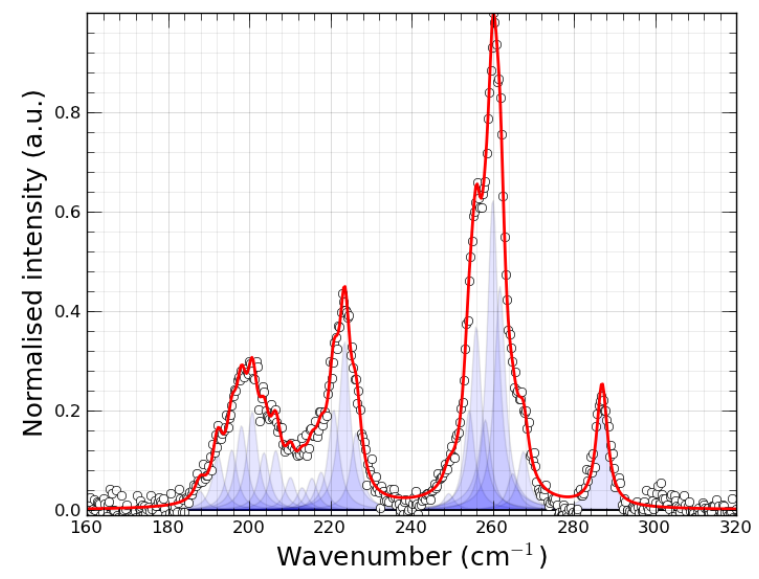

(b) Manual peak fitting

Figure 5.8: Deconvolution of a RBM spectrum of a thin film using a typical GUI software with lorentzians.

A typical fitting routine begins with a manual inspection of the spectrum using visual clues like peak shoulders and bumps to indicate components. A graphical user interface is often used to select the position of the component peaks. Once the user is satisfied that all the component peaks are included, constraints are applied on each peak and the minimisation algorithm is run. Minimisation routines that utilise the Levenberg-Marquadt algorithm are particularly sensitive to initialisation parameters and it is up to the user to ensure that all initial values are not too far away from the optimal value. Nonetheless, fitting spectra with three free parameters 

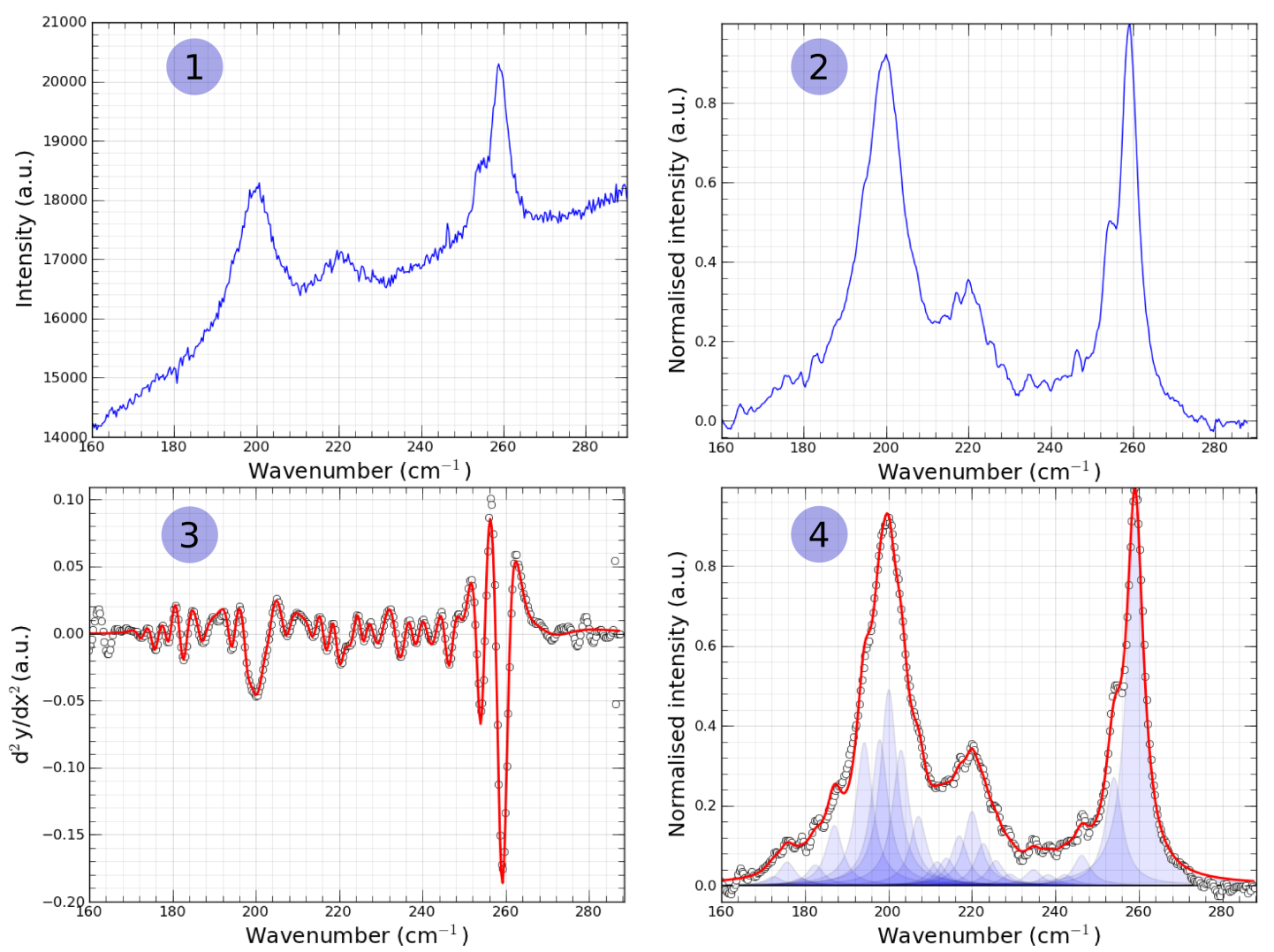

Figure 5.9: Flowchart showing the analysis procedure from raw data to the final fit.

peak becomes progressively less stable and accurate as the number of components increase. When confronted with a spectrum with very few clear shoulders (Figure 5.8), both user and software run into the same trouble highlighted above. Each peak now no longer corresponds to a single nanotube of some chirality $(n, m)$ and there is little physical significance in such a fit.

In order to track the parameters of individual RBMs such as the linewidth, position or intensity, across different samples, we chose to fit the RBM spectra with a slightly more complex procedure than the typical one described in the above paragraph. The flowchart in Figure 5.9 shows that the analysis follows a series of steps from treating the data to the final fit.

\section{Raw spectrum}

2. Spectrum smoothed using a Savitzky-Golay filter with a window of 15 points and order 3. A linear background is subtracted from the raw data.

3. The second derivative is calculated from the smoothed data in 2. after an additional smoothing using a standard triangular-window moving average. This extra smoothing step ensures that extraneous noise features are not picked up by the second derivative. The second derivative is then fit with a user-specified combination of peaks. The plot shown here has been fit to 12 component gaussians. The fit is engineered such that the second calculated second derivative fit to the calculated second derivative of the 12 components. 
4. The peak positions fitted in the above step and a single linewidth for all peaks are used to constrain the fit to the spectrum. The fitted spectrum is further analysed for negative (unphysical) intensities. In the case of negative intensities (which usually occur for very small features), the peak is removed from the list and then the spectrum refitted. This step is both a check of the fit quality and stability. In the case of a fit with negative intensity components, the second fitting round rejects these peaks and refits with the remaining peaks. When the fit contains no negative peaks, this second minimisation checks the stability of the original fit.

The benefit of the fitting procedure described here is the quantity of information that is suddenly available for further analysis compared to the traditional single step fitting. As per the original intentions, the RBM spectra of heavily roped SWNTs can be deconvoluted into component peaks that largely correspond to individual tubes. Unambiguous assignments with unique $(n, m)$ are not likely for all the deconvoluted peaks in the case of our chosen laser line but could be achieved with lines that excite a smaller population of tubes (e.g. $514.5 \mathrm{~nm}$ ).

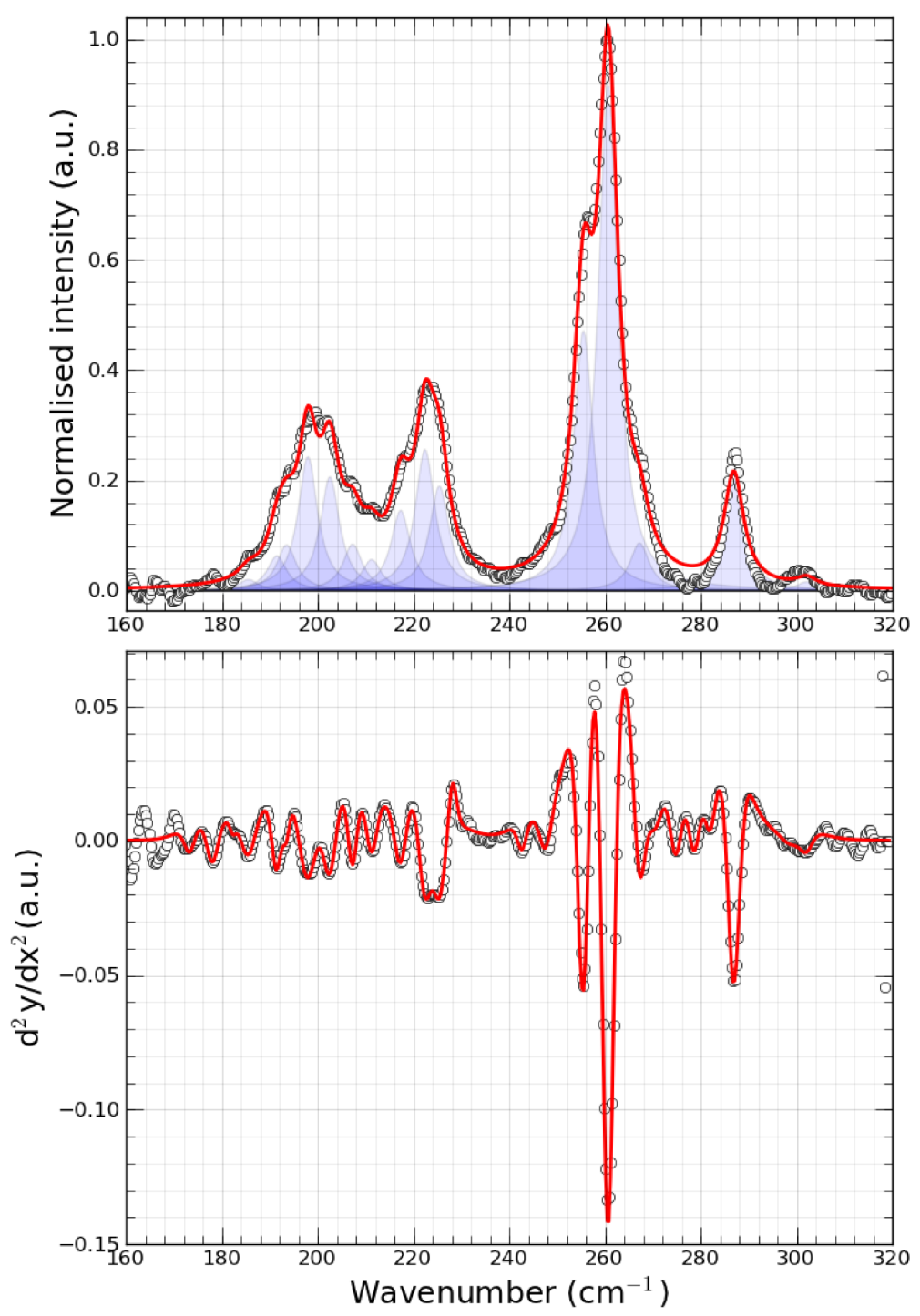

Figure 5.10: Comparing deconvoluted spectra with the second derivative. 


\subsubsection{Fitting constraints}

In a situation where a spectrum is a superposition of a large number of components, a stable fit is unlikely. As mentioned before, this is especially difficult in the case of spectra with very small bumps indicating the presence of the components. Constraining the peak positions is one route to stabilise the fit. Another is to constrain the linewidth of all the peaks in resonance to a single value - an option that has been suggested by others. Other studies also advocate the use of a single linewidth to fit all the RBM peaks in a given spectrum [102, 103]. This is despite the knowledge of wide fluctuations in the RBM frequency of a single SWNT as well as the likely chirality dependence.

However, using a single fitted linewidth requires all the peaks to be resolved in the second derivative. Peaks that are unresolved in the second derivative will increase the effective linewidth for all the other peaks. For example, the peak at $286 \mathrm{~cm}^{-1}$ in Figure 5.10 is not a single peak but a doublet, as evidenced by the asymmetry of the second derivative and indicates that the separation between the doublet is less than $80 \%$ of the peak linewidth. The doublet at $224 \mathrm{~cm}^{-1}$ is just one step better than the one at $286 \mathrm{~cm}^{-1}$ as both peaks are resolved separately, and can be fitted easily. Thus, it is crucial that the experimental conditions are chosen such that single peaks are clear in the second derivative. The following section will illustrate that the laser power is an important parameter for tuning resolution in the second derivative.

\subsubsection{Fitting accuracy}

Figure 5.11 shows the fitted frequency components of a thin film RBM spectrum (as red diamonds) overlaid on an experimental Kataura plot. The Kataura plot is an excellent comparison to our data as the samples measured by Maultzsch et al. used the same unpurified HiPCO nanotubes. The proximity of the red diamonds to the Kataura plot points indicate that most of the fitted frequency components can be associated with a unique nanotube. The peaks that can be unambiguously identified are given in Table 5.1 along with several properties of the tubes including their type (metallic or semiconducting), diameter, transition, $(n, m)$ indices and Raman susceptibility. All these values are taken from the reference of Maultzsch et al. 994.

However, there are two major exceptions to agreement between the fitted data and the Kataura plot. The first is the region between 180 and $205 \mathrm{~cm}^{-1}$ (highlighted in blue). From the shaded resonance window of $\sim 160 \mathrm{meV}$, we can see that there are too many peaks that are too close together within the highlighted blue region. Given that RBM peaks can appear and change position due to a variey of environmental factors such as temperature or bundling, we cannot derive accurate information from a composite with too many closely-spaced components. For this reason, most of the analysis in this chapter is focused on the 220 and $250 \mathrm{~cm}^{-1}$ composites. The second problem is the under-fitting or over-fitting of the $220 \mathrm{~cm}^{-1}$ composite. 


\begin{tabular}{|c|c|c|c|c|c|}
\hline$\omega_{\text {peak }}$ & $(n, m)$ & Type & Diameter $(\AA)$ & $E_{i i}(\mathrm{eV})$ & Suscept. \\
\hline \hline 183 & $\mathrm{M}$ & $(11,8)$ & 12.94 & 1.936 & 1.9 \\
\hline 189 & $\mathrm{M}$ & $(12,6)$ & 12.44 & 1.948 & 1.9 \\
\hline 212 & $\mathrm{M}$ & $(11,5)$ & 11.11 & 2.084 & 1.7 \\
\hline 217 & $\mathrm{M}$ & $(12,3)$ & 10.77 & 2.075 & 2.6 \\
\hline 221 & $\mathrm{M}$ & $(13,1)$ & 10.60 & 2.057 & 1.5 \\
\hline 242 & $\mathrm{~S}$ & $(9,5)$ & 9.63 & 1.84 & - \\
\hline 252 & $\mathrm{~S}$ & $(10,3)$ & 9.24 & 2.031 & 9.8 \\
\hline 256 & $\mathrm{~S}$ & $(11,1)$ & 9.03 & 1.945 & 3.6 \\
\hline 264 & $\mathrm{~S}$ & $(7,6)$ & 8.83 & 1.909 & 2.6 \\
\hline 277 & $\mathrm{~S}$ & $(8,4)$ & 8.29 & 2.10 & 0.3 \\
\hline 284 & $\mathrm{~S}$ & $(7,5)$ & 8.18 & 1.915 & 18.3 \\
\hline \hline
\end{tabular}

Table 5.1: Important physical parameters for the set of uniquely identifiable SWNTs resonant at $1.96 \mathrm{eV}$ from a starting sample of unpurified HiPCO nanotubes.

Unlike the clear shoulders in the $250 \mathrm{~cm}^{-1}$ composite, the $220 \mathrm{~cm}^{-1}$ composite is broad and partly convoluted with the $200 \mathrm{~cm}^{-1}$ composite. Given this poor resolution in the spectral data, the fitting routine does not always converge on the right number of peaks. However, the values that are fitted are usually quite close to expected components.

\subsection{Methodology: statistical RBM analysis}

Analysis of the RBMs from the mapping datasets could not be carried out using the high resolution method of deconvoluting the entire RBM spectrum (illustrated in Section 5.3). This method is inappropriate since the size of the mapping datasets (between 100-400 spectra) is too time consuming. Furthermore, the amount of information discerned from the entire RBM spectrum is unnecessary for the simple theses that are explored in the spatial mapping experiments. Hence, we have focused on simply deconvoluting the $250 \mathrm{~cm}^{-1}$ composite peak. This simpler alternative actually contains a substantial amount of information on the degree of bundling and local heating effects on the resonance window, RBM frequency, linewidth and intensity. These parameters will be explored in the following section and Chapter 6.3.

The deconvolution of the $250 \mathrm{~cm}^{-1}$ composite usually requires three lorentzian components since the two tubes from the $(11,1)$ family - 252 and $256 \mathrm{~cm}^{-1}$ and the tube from the $(10,0)$ family - $264 \mathrm{~cm}^{-1}$ - are close to resonance (within $100 \mathrm{meV}$ ). In the case of higher temperature spectra, four to five lorentzians were required due to the emergence of the $242 \mathrm{~cm}^{-1}$ tube from the $(12,0)$ family and the $272 \mathrm{~cm}^{-1}$ tube from the $(10,1)$ family. The tubes from the $(10,1)$ family are actually quite far from resonance $\left(E_{i i} \sim 2.4 \mathrm{eV}\right)$ but the effect of a large Raman cross-section and the downshifting of the transition energies with temperature brings them into resonance at high temperatures. In saying this, the relatively low intensity and infrequent appearance of this nanotube family means it can usually be ignored. The fitting was carried 


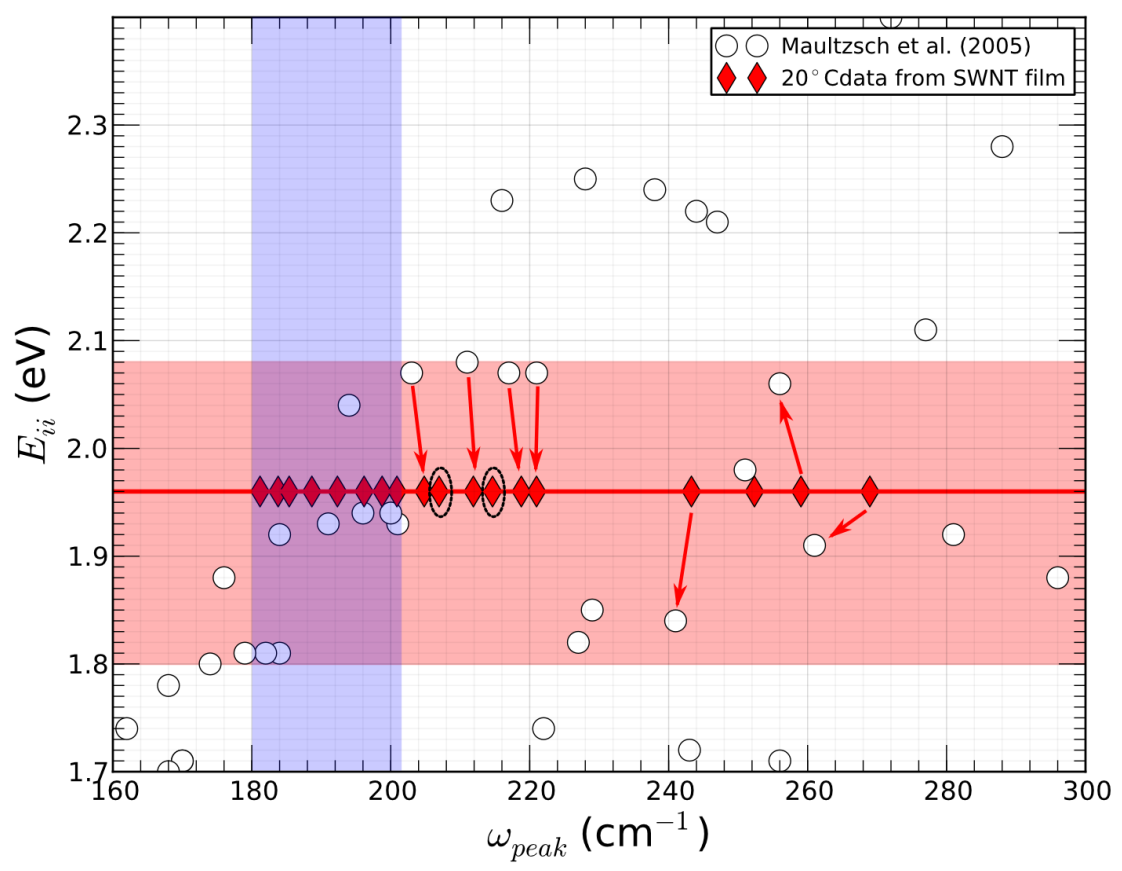

Figure 5.11: Top: Experimental Kataura plot obtained from HiPCO tubes (Maultzsch et al.). The red diamonds are the deconvoluted peaks of a thin film taken at $20{ }^{\circ} \mathrm{C}$. The blue band indicates the range of data for which unambiguous assignation of $(n, m)$ is difficult.

out with the same logic of constraining the linewidth (as described in Section 5.3.2). However, since the mapping did not always cover regions with nanotubes, a limit on the observed counts per second (cts/s) had to be imposed on the datasets to differentiate between noise and real data. This limit corresponded to a background-corrected intensity of 100-200 cts/s. The fitted values of the peak frequencies, effective linewidths and intensities were then used for additional analyses.

The peak frequencies were examined in detail by plotting into histograms. Bin widths were chosen to be between $0.1-0.3 \mathrm{~cm}^{-1}$ depending on the dataset. Once binned, the two dimensional dataset was fit with a simple gaussian: $I=I_{0} \exp \frac{-\left(x-x_{0}\right)^{2}}{2 \sigma^{2}}$. The parameters $x_{0}$ and $\sigma$ give the mean peak frequency and the standard deviation of the distribution of frequencies.

Establishing the true peak frequency of one SWNT within a bundled region is subject to many limitations, including deconvolution, peak resolution, spectrometer calibration and drift. Since deconvolution has been addressed by using a set of methods illustrated in Sections 5.3 and 5.4, the spectrometer calibration issues are the main limitation affecting the band of uncertainty about the true peak frequency. The following section explains how we have established a simple method for obtaining deconvoluted peak frequencies with the major source of uncertainity as the grating resolution. 


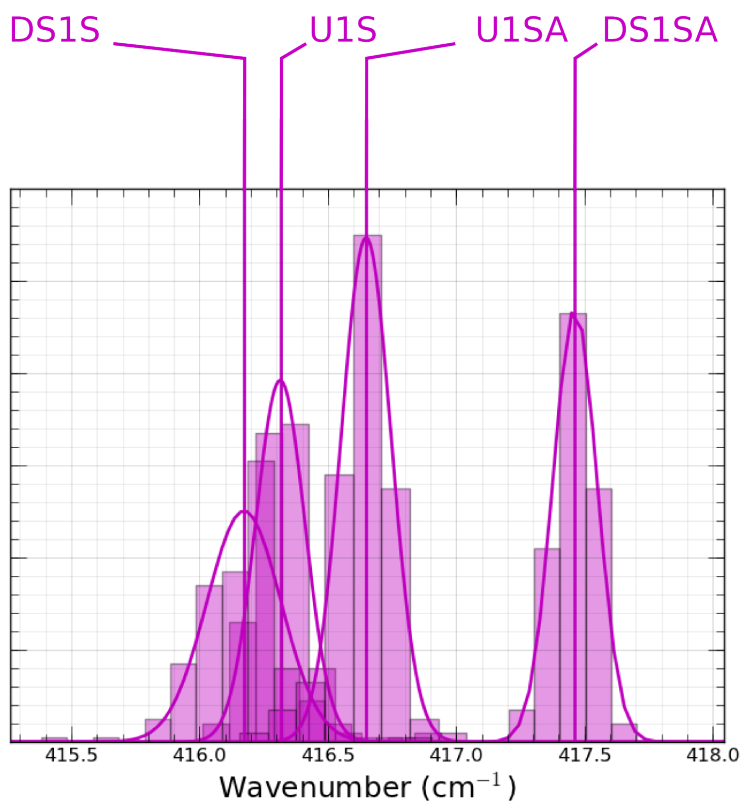

Figure 5.12: Sapphire frequencies from SWNT thin film mapping dependence showing considerable variation in the peak position.

\subsubsection{Calibration}

The typical method for calibrating the spectrometer is with an external standard such as silicon. The parameters of the grating position are adjusted such that the silicon peak maximum is at $520 \mathrm{~cm}^{-1}$. However, this method doesn't allow for a constant adjustment with uncertainties associated with the grating position and the gradual drift of the spectrometer due to thermal effects that occurs over the course of a day. In these cases, it is useful to have an internal calibration from the substrate or a plasma line within the acquisition window that allows for an independent comparison standard for multiple measurement sessions. Since we chose c-plane sapphire as a substrate, calculating an internal calibration is a relatively simple task. The sapphire $\left(A_{1 g}\right)$ peak at $416.7 \mathrm{~cm}^{-1}$ [104, 105] is always included in the spectral window when acquiring RBM data. However, a few details need to be confirmed before the sapphire peak position can be used to correct for drift and grating position uncertainty. Figure 5.12 shows the spread in the peak position histograms from four SWNT thin film samples. This variation is likely because of the inherent spectrometer drift rates on different days. Given this variability in the sapphire peak, we need to establish the peak frequency that will be used as a calibration from a control sample taken shortly after calibrating the spectrometer.

A plain piece of sapphire was used to obtain the room temperature value of the $A_{1 g}$ peak. Prior to the measurements on sapphire, the spectrometer was adjusted such that the silicon peak was exactly at $520 \mathrm{~cm}^{-1}$ (Figure 5.13a). Natural variations in the $A_{1 g}$ peak frequency were checked for using spatial and axial dependence measurements on both the polished and unpolished sides. Figure 5.13 shows all the relevant measurements for the sapphire calibration. It is apparent from the room temperature measurements that the $A_{1 g}$ peak frequency falls quite tightly around $416.5 \mathrm{~cm}^{-1}$. However, the temperature dependence measurements (data 


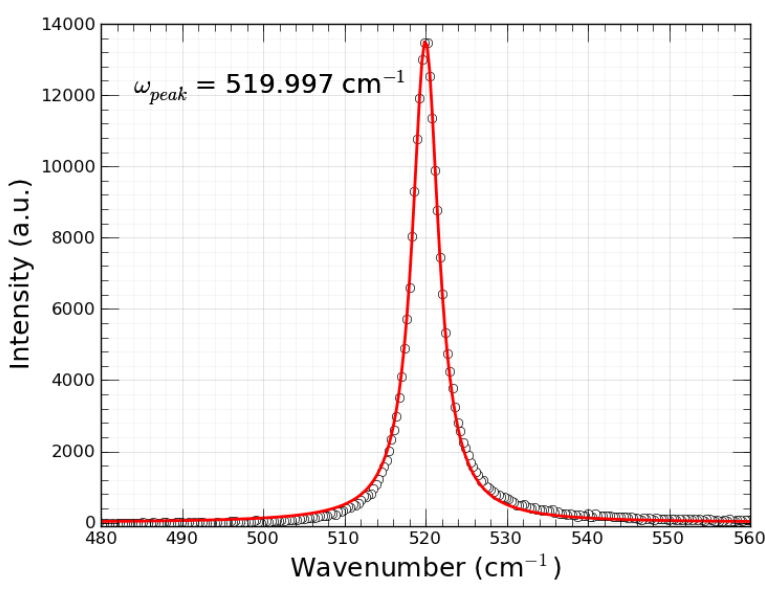

(a) Silicon

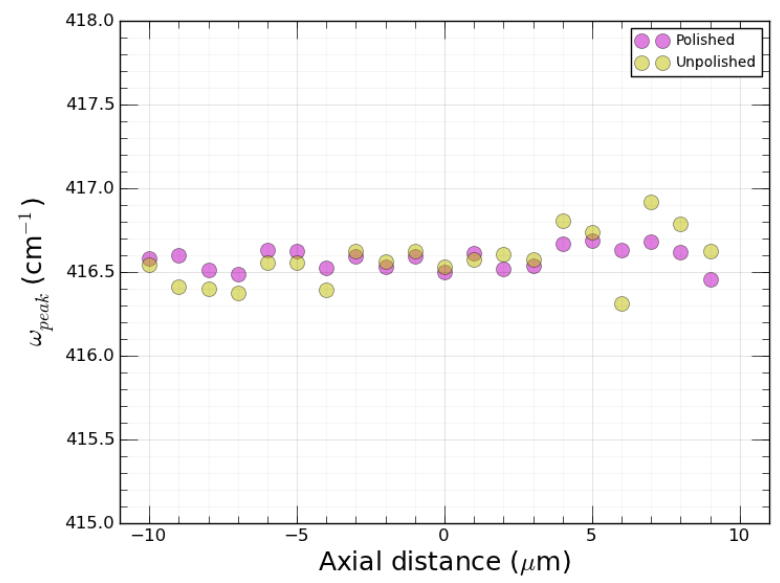

(c) Axial dependence
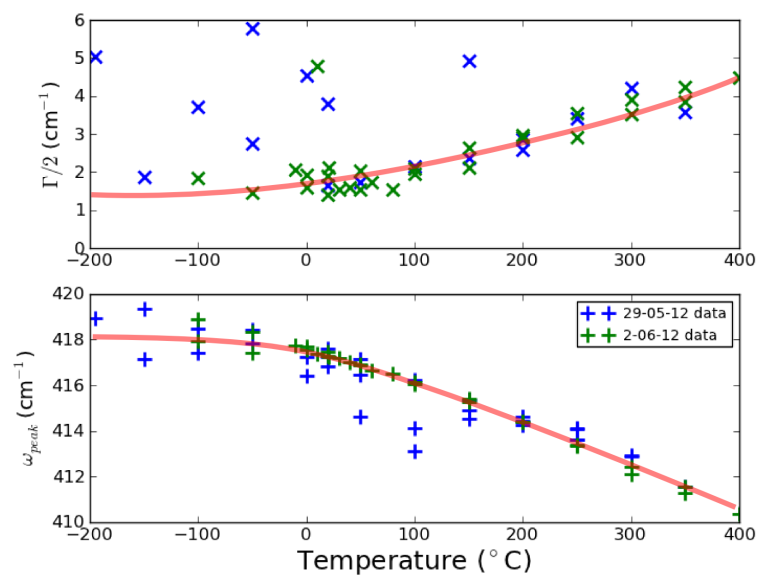

(b) Temperature dependence

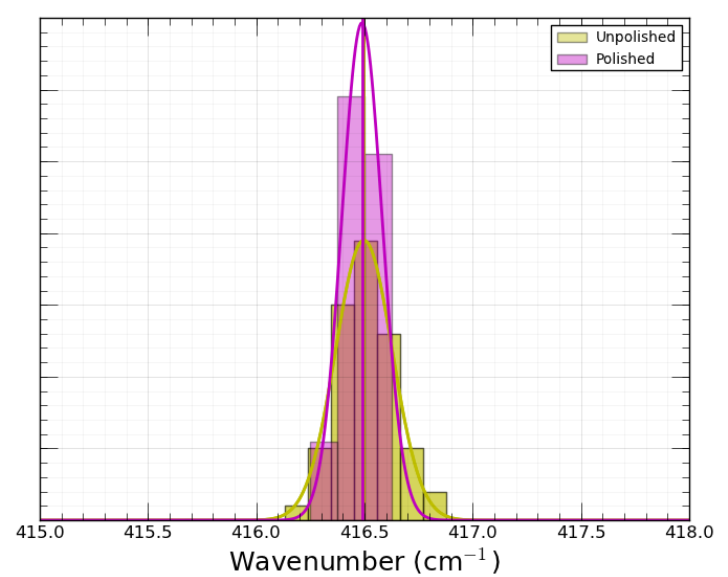

(d) Spatial dependence

Figure 5.13: (a) Spectrometer calibrated so that the silicon peak is exactly at $520 \mathrm{~cm}^{-1}$. (b)Temperature dependence of the sapphire peak isolated from the temperature dependence data of SWNT films on two separate days of measurement. The transluscent red line is a visual aid to see the general trend of the temperature dependence data. Axial dependence (c) and spatial dependence $(\mathrm{d})$ of the sapphire peak on the polished and unpolished sides of a clean substrate.

extracted from the temperature dependence of SWNT thin films) show that the frequency vaires between $417-418 \mathrm{~cm}^{-1}$. This upshift probably corresponds to noisy signal since the focus is no longer on the sapphire but further away on the SWNT thin film. Evidence for noisy data with positive displacement of the objective is also seen in the axial dependence data in Figure 5.13c. Thus, from the simple measurements of the axial and spatial dependence using the specific grating, spacer and objective configurations (discussed previously), the sapphire peak frequency can be assigned as $416.5 \pm 0.1 \mathrm{~cm}^{-1}$. This value can now be used to calibrate the different spatial maps of SWNT films.

The dispersion seen in the sapphire sample is quite small compared to the dispersion of frequencies seen in SWNT networks (see the following section). To ensure that the dispersion 


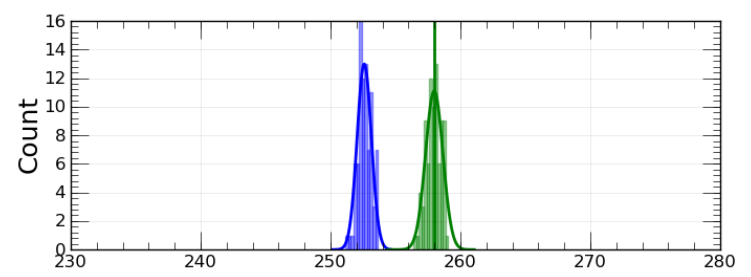

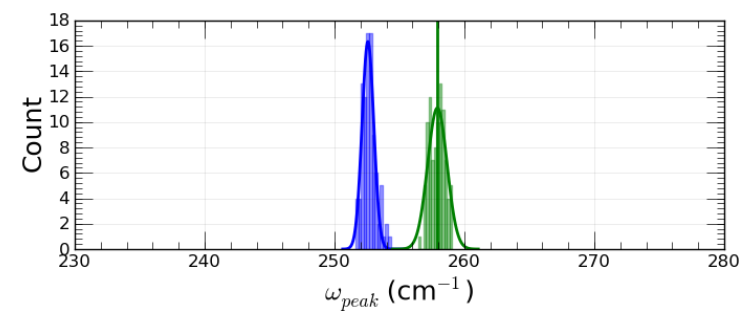

(a) Frequency histograms

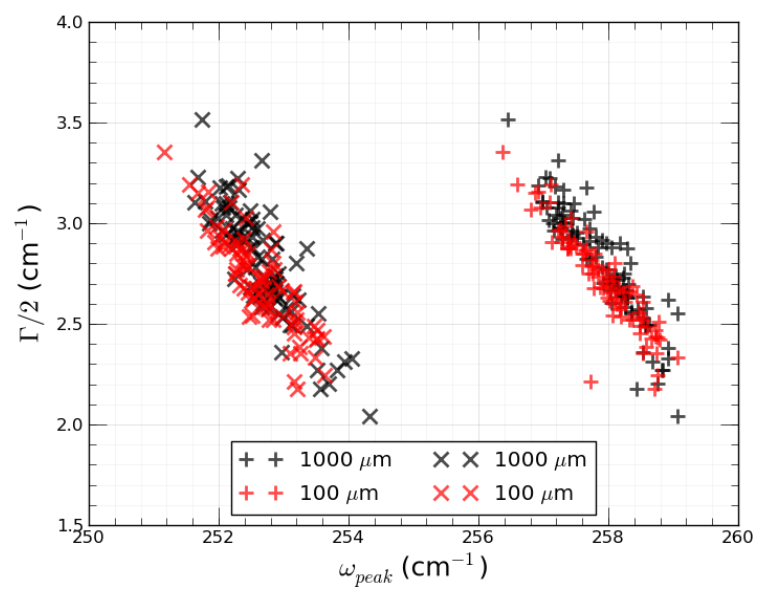

(b) $\Gamma$ vs. $\omega_{\text {peak }}$

Figure 5.14: (a) Histograms of the deconvoluted peaks within the $250 \mathrm{~cm}^{-1}$ composite at two pinholes: $100 \mu \mathrm{m}$ (top plot) and $1000 \mu \mathrm{m}$ (bottom plot). (b) Linewidth vs. peak frequency (252 and $256 \mathrm{~cm}^{-1}$ peaks).

isn't an artifact of a misaligned beam/broadening from a too-large pinhole, spatial maps from a SWNT sample (U2SAN2; Table 5.2) were collected at two different pinholes (1000 and 100 $\mu \mathrm{m})$. Figure $5.14 \mathrm{a}$ shows the dispersion in frequency persists regardless of pinhole size. To check if it is due to local heating, Figure 5.14b shows the peak frequencies from Figure 5.14a plotted against the effective linewidth. The linear correlation between the two parameters is almost identical for the fully open and constricted pinhole data. Thus, the frequency dispersion is directly related to the frequency softening that occurs due to local heating. This pinhole dependence experiment was carried out at the outset and only included in full here due to the shared context of local heating. In fact, the success of the singe peak deconvolution technique was dependent upon the pinhole having a negligible effect. If the pinhole size was correlated with the frequency dispersion, a very small pinhole would have been necessary for the experiments; a factor that would have considerably slowed down data collection.

\subsubsection{Analysing data from spatial maps}

Two sets of data analysis were carried out for each SWNT sample. The first involved fitting the $250 \mathrm{~cm}^{-1}$ peak to anywhere between three and five lorentzian components. After deconvolution, the peaks from a given spectrum were corrected according to the shift of the sapphire peak from the established peak position of $416.5 \mathrm{~cm}^{-1}$. The values of the peak frequency were then plotted as a histogram for each peak and each histogram fitted with a gaussian distribution. For a clear visual trace of variations in the mean peak frequency, this value is highlighted using a solid vertical line of the same colour as the histogram. The peaks are colour coded as follows: $242 \mathrm{~cm}^{-1}$ (cyan), $252 \mathrm{~cm}^{-1}$ (blue), $256 \mathrm{~cm}^{-1}$ (green), $264 \mathrm{~cm}^{-1}$ (red) and $272 \mathrm{~cm}^{-1}$ (magenta). The second analysis calculated the normalised integrated intensities of the $220 \mathrm{~cm}^{-1}$ and 250 


\begin{tabular}{|c|c|c|c|c|}
\hline Name & As-prepared state & Film thickness & Treatment & Name: post-treatment \\
\hline \hline U1S & Wet-sprayed & thin & Annealing in air & U1SA \\
\hline U2S & Wet-sprayed & thick & Annealing in nitrogran & U2SAN2 \\
\hline U3S & Wet-sprayed & thin & Annealing in air & U3SA (not tested) \\
\hline DS1S & Dry-sprayed & thin & Annealing in air & DS1SA \\
\hline \hline
\end{tabular}

Table 5.2: List of samples.

$\mathrm{cm}^{-1}$ composites for each spectrum. Integrated intensities for the $220 \mathrm{~cm}^{-1}$ and $250 \mathrm{~cm}^{-1}$ composites were calculated from the raw data between 205 and $235 \mathrm{~cm}^{-1}$, and between 235 and $275 \mathrm{~cm}^{-1}$ respectively.

\subsection{Characterisation: effects of the film fabrication pro- cess}

The remainder of this chapter concerns the thin film fabrication process and using Raman spectroscopy to understand the effects of solvent removal. In a previous study [52], we showed that the remnant solvent in as-prepared samples can be removed by a simple annealing process. From combined TGA and DSC, the solvents evaporated by $100{ }^{\circ} \mathrm{C}$. With this information, 'dryspraying' of SWNT networks was achieved by spraying the SWNT dispersion onto a substrate heated to $110{ }^{\circ} \mathrm{C}$. In this brief characterisation, we study the RBM spectra of: samples still containing solvent, dry-sprayed samples and samples annealed at a higher temperature of 320 ${ }^{\circ} \mathrm{C}$.

Four basic samples were prepared by spraying an SWNT dispersion (Chapter 4.2.1) for a few seconds onto sapphire. All samples except one were prepared by using a spray time of $10 \mathrm{~s}$. The dry-sprayed sample required spraying onto a substrate heated at $110{ }^{\circ} \mathrm{C}$ while two unannealed samples were prepared without heating (wet spraying). A 'thick' sample was fabricated by wet spraying for around two minutes. Raman spectra focusing on the RBMs were collected as a spatial map across a region of approximately $100 \mu \mathrm{m} \times 100 \mu \mathrm{m}$ and a minimum step size of $5 \mu \mathrm{m}$ with a $1 \mathrm{~s}$ acquisition time at each spot. Following this initial data collection, the samples were annealed in a tube furnace (under ambient atmosphere or $\mathrm{N}_{2}$ ) at $320{ }^{\circ} \mathrm{C}$ for five minutes. The nomenclature of the sample names is a set of initials that follow as: 'type, sample number, substrate, treatment' (Table 5.2). So, the first unannealed sample on sapphire which was subsequently annealed in air is 'U1SA'. If the annealing was carried out in nitrogen instead, the name becomes 'U1SAN2'. Maps of the RBMs were collected after the treatment using the same conditions described above. The data was analysed according the method detailed in Section 5.4 .2 . For simplicity, we have only analysed the dominant $256 \mathrm{~cm}^{-1}$ peak.

It is important to note that the component RBMs don't vary much between different unan- 


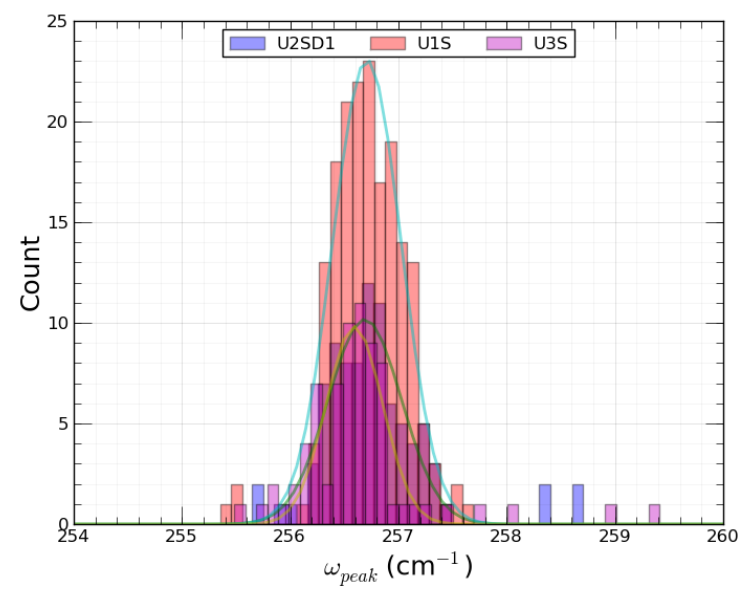

(a) Unannealed Samples

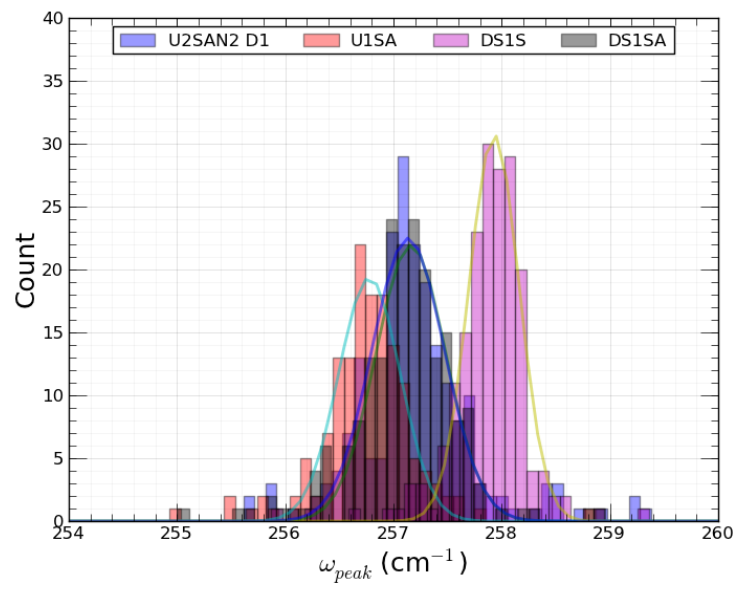

(b) Annealed samples

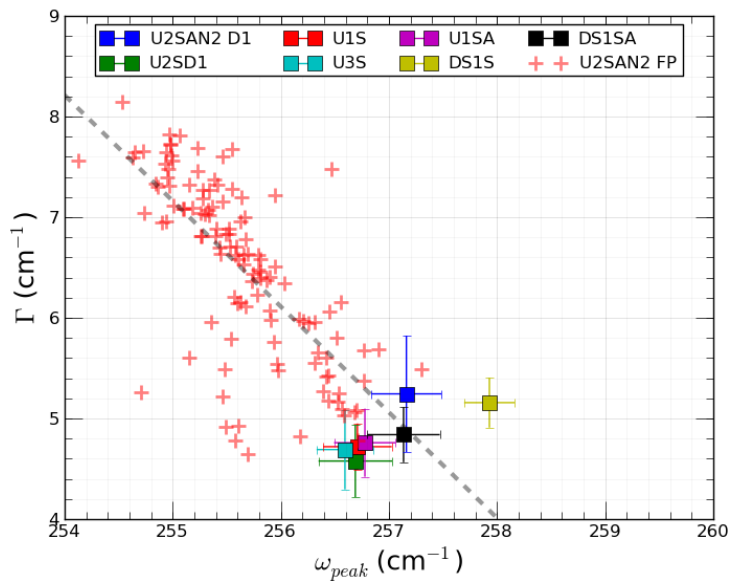

(c) Summary

Figure 5.15: Histograms of peak positions for unannealed (a) and annealed (b) samples. (c) Trends in the linewidth vs. peak frequency for the two sample types: annealed and unannealed. The dashed line is the linear fit to the full power data of U2SAN2.

nealed samples despite slight variations in sample preparation like the state of the spraying solution or the spraying time. For example, samples U2S and U3S were prepared with different thicknesses using the same solution. The former was prepared by spraying the solution for 3 minutes while the latter was made with spray that lasted around 10 seconds. Figure $5.15 \mathrm{a}$ shows that the peak position of the $256 \mathrm{~cm}^{-1}$ composite is consistent across the different unannealed samples. The one caveat to consistency seen in the unannealed samples is the power dependence of the thick sample. Unlike the other two thin samples, the thick sample shows a marked difference when probed with a full power laser (see Chapter 6.3). At lower power, the spectra are comparable to the full power spectra of the thin samples.

The peak positions remain in a tight cluster upon annealing (Figure 5.15b) though the mean peak position of the dry sprayed sample is around two standard deviations away from the cluster of the high temperature annealed samples. Figure $5.15 \mathrm{c}$ which plots the RBM peak 
linewidth plotted against the peak frequency of the $256 \mathrm{~cm}^{-1}$ peak for all the samples shows the clustering and the distinct separation of the dry sprayed sample. From the extrapolation of the trendline demonstrating pure local heating (thick annealed sample at full power), the cluster around $257 \mathrm{~cm}^{-1}$ sits close to the expected value of $\omega_{\text {peak }}$ when the linewidth is around $4.5 \mathrm{~cm}^{-1}$ (approximate limit of bundled samples seen in Section 6.2.3).

These experiments show that all the high temperature annealed samples and unannealed samples fall in a tight cluster about the linewidth and peak position expected at ambient temperature. The outlier of this cluster is the dry sprayed sample which exhibits a higher peak position to the other samples. The degree of ambient doping does not typically affect the RBM frequencies [106]. Furthermore, the local density of nanotubes has a strong correlation with the degree of local heating (Chapter 6.3). Hence, we can surmise that the upshift seen for the DS1S sample is due to the probed region: where nanotube coverage is extremely sparse and local heating by the laser is smaller compared to the other samples.

\subsection{Conclusions}

We have described two unique routes for analysing RBM spectra from bundled nanotubes. The first route uses an interactive fitting algorithm that allows for a high resolution deconvolution of complex RBM spectra. The second route is useful for analysing large volumes of data. We use this technique for a brief study of the thin film fabrication process. Most of the samples are clustered around a linewidth of $4.6 \mathrm{~cm}^{-1}$ and a peak position of $257 \mathrm{~cm}^{-1}$. The spatial sensitivity of this technique provides some preliminary insight into the effects of local nanotube density and local heating. 


\section{Chapter 6}

\section{Temperature-dependent Raman spectroscopy of SWNT networks}

\subsection{Introduction}

Despite prolific literature on the correlation between the RBMs and various physical parameters e.g. doping, diameter distribution, degree of bundling; the temperature dependence of the RBMs is limited to a few studies. Of these studies, the majority only consider the temperature dependence of RBM frequencies [107, 108, 109, 110, 111, 112]. Only two studies have directly measured the temperature dependence of the RBM linewidth [113, 114] while the RBM intensity is not directly measured; its dependence on temperature is only inferred from the expected trend in $E_{i i}(\mathrm{~T})$ [97, 76]. In this section, we present the temperature dependence of the full RBM lineshape - frequency, linewidth and intensity - for the majority of unambiguous SWNTs resonant at $1.96 \mathrm{eV}$ (Table 5.1). The effects at ambient temperature are compared with local heating by the laser. The results of this detailed study provide insight on fundamental issues that are yet to be resolved: the contribution of anharmonicity to the RBM frequency and linewidth and, the convolution of bundling and local heating effects.

\subsection{Global heating}

As noted in the previous chapter, the RBM spectra from bundled regions can be complex with poor resolution of peak features. Figure 6.1 shows the RBM spectra at three temperatures. Even at $20{ }^{\circ} \mathrm{C}$, the sharpness of component peaks within the composites in our samples is considerably less than the RBM spectra of bundled SWNTs from literature [107, 108]. This reduced resolution in our SWNT films points to: (i) a larger linewidth and (ii) a larger number of resonant nanotubes due to a broadened resonance window. These effects are consistent with a sample comprised of larger SWNT bundles than those in the literature. Thus, deconvolution of the RBM spectra were carried out using the high resolution fitting routine described in Chapter 5.3 . 


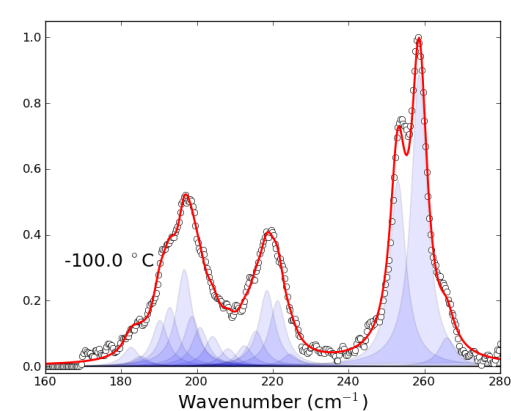

(a) $-100{ }^{\circ} \mathrm{C}$

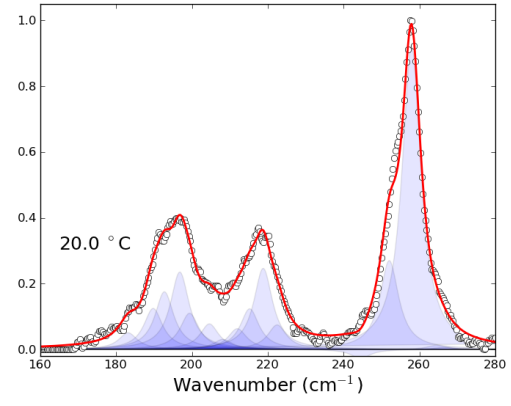

(b) $20{ }^{\circ} \mathrm{C}$

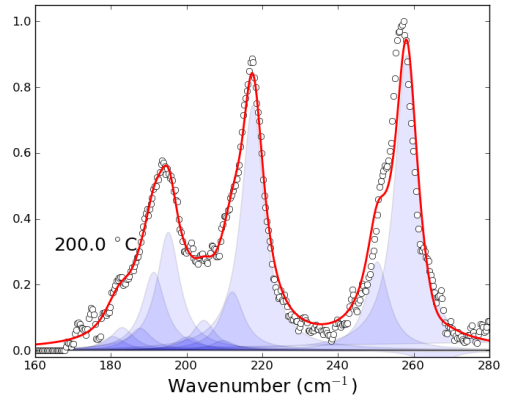

(c) $200{ }^{\circ} \mathrm{C}$

Figure 6.1: Temperature dependence of a bundled region of a SWNT thin film.

\subsubsection{Data collection}

The data collection for the temperature dependence study was relatively straightforward. Two SWNT thin films (U1SA and U2SNA2; see Table 5.2) that were sprayed and subsequently annealed at $300{ }^{\circ} \mathrm{C}$ for 5 minutes were placed in a Linkam cryostat. The sample space was constantly under a flow of nitrogen to prevent combustion of nanotubes. Measurements were taken approximately every $50{ }^{\circ} \mathrm{C}$ though the step size was shortened to $20{ }^{\circ} \mathrm{C}$ around room temperature. The laser spot was moved to a given spot (we tried to keep this spot consistent for most of the measurements on a given day) and refocused with the aid of the built-in optical microscope prior to data acquistion at a given temperature. Data was collected at full power with an acquisition time of $1 \mathrm{~s}$. Typically, data was collected in the following order: $20^{\circ} \mathrm{C}$ to $-195{ }^{\circ} \mathrm{C}$ to $400{ }^{\circ} \mathrm{C}$ and finally back to $20{ }^{\circ} \mathrm{C}$. A total of $\sim 65$ spectra were collected in this temperature range. Each spectrum was deconvoluted using the high resolution, second derivative fitting routine described in Chapter 5.3. The final peak positions, intensities and linewidth were exported to a txt file in order to analyse the temperature dependence of each parameter.

\subsubsection{Peak frequency}

This section presents the temperature dependence of the RBM frequencies from bundled HiPCO SWNTs. The temperature dependence of the peak frequency is due to anharmonic effects with components due to (i) volume effects and/or (ii) phonon decay processes. In some cases one mechanism dominates over the other e.g. the temperature dependence of the $\mathrm{A}_{1 g}$ phonon frequency in $\mathrm{CuO}$ is dominated by phonon decay processes alone (Chapter 8). The temperature dependence of RBM frequencies has only been considered in a small number of studies. Previous studies include those of Meletov et al. [107] on small bundles of arc discharge nanotubes; Uchida et al. [108], Zhang et al. [109] and Zhou et al. [110] on CVD grown nanotubes; and Karachevtsev et al. [111] and Raravikar et al.[112] on bundled HiPCO nanotubes. Both the 
relevant studies on HiPCO nanotubes only looked at a small subset of the full range of possible data. Karachevtsev et al. carried out their studies at two temperatures while Raravikar et al. looked at the temperature dependence of only two RBMs; both large diameter tubes. Thus, we are limited to the studies on individualised CVD tubes by Zhang et al. [109] and Zhou et al. [110.

RBM peak frequencies from both the high resolution and single composite deconvolution methods are discussed below. For the former, only the peak frequencies that could be unambiguously associated with nanotubes were extracted e.g. $283(7,5), 264(7,6), 256(11,1)$, $252(10,3), 221(13,1), 217(12,3), 212(11,5), 189(12,6)$ and $183(11,8)$. The montage of plots in Figure 6.2 shows the temperature dependence for these nine RBMs. The margin of fitting error is comparable to the temperature dependence of the frequency in all but the RBMs with frequencies above $250 \mathrm{~cm}^{-1}$. The degree of noise in the peak frequencies not only obliterates any underlying temperature dependence but also indicates the limitations of the high resolution deconvolution. The large number of closely-spaced resonant SWNTs between 180 and $220 \mathrm{~cm}^{-1}$ blur the RBM spectrum too much. The composite at $220 \mathrm{~cm}^{-1}$ is particularly problematic. The composite is not clearly isolated from the $200 \mathrm{~cm}^{-1}$ composite, and it can contain 3 to 5 component peaks. The major problem is the relative intensity of the three main components; they are too close together and thus have no visible shoulders. As a result, the fitting of the low wavenumber region is at the mercy of the various instabilities associated with fitting poorly defined peaks. Above $250 \mathrm{~cm}^{-1}$, the number of resonant tubes is fairly low and the RBMs of this region can be fitted with a high degree of accuracy. Figures 6.2c and $6.2 \mathrm{~d}$ show data with a relatively small level of noise compared to the variation with temperature.

Figure 6.3 presents a visual comparison of the component peaks of the $250 \mathrm{~cm}^{-1}$ composites calculated using single composite (Figure 6.3a) and full spectrum (Figure 6.3b) deconvolution methods. Despite small differences in the number of fitted components, the temperature dependence of the two main peaks from the $250 \mathrm{~cm}^{-1}$ composite are reproduced by both the methods. The number of peak components does not seem to affect the majority of the data since the $264 \mathrm{~cm}^{-1}$ peak is both weak and further away from resonance compared to the 252 and $256 \mathrm{~cm}-1$ peaks.

The linear correlation between frequency and temperature is typically interpreted in terms of volume effects and phonon decay processes. But, volume contributions to the frequency downshift in graphene and related materials are considered negligible since the thermal expansion coefficient is both small and negative. The radial thermal expansion coefficient of SWNTs is measured as $-1.5 \times 10^{-6} \mathrm{~K}^{-1}$ [115]. The sign of the thermal expansion gives a stiffening of the frequency with increasing temperature [115] - opposite to the observed trend - suggesting anharmonicity dominated by phonon decay processes. However, most temperature dependence studies of the RBM frequency reject this mechanism [116, 109, 117] in favour of the mechanism 


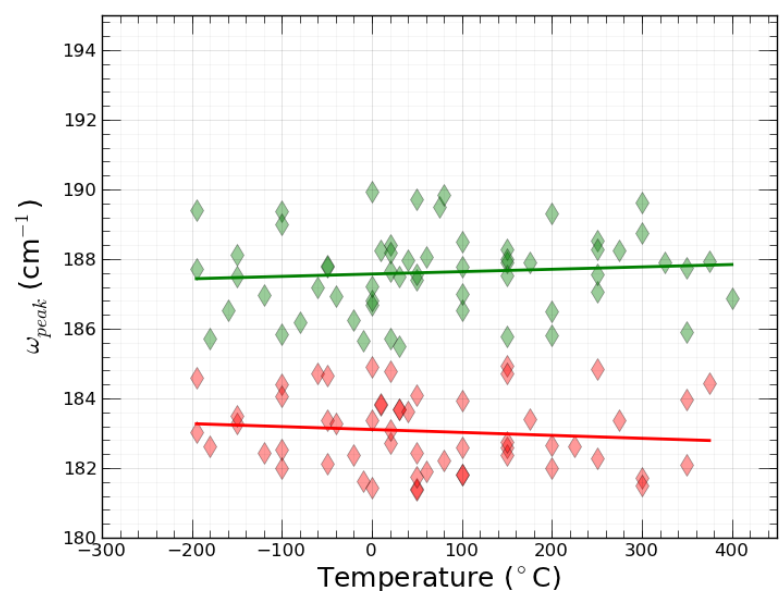

(a) 183 and $189 \mathrm{~cm}^{-1}$ peaks

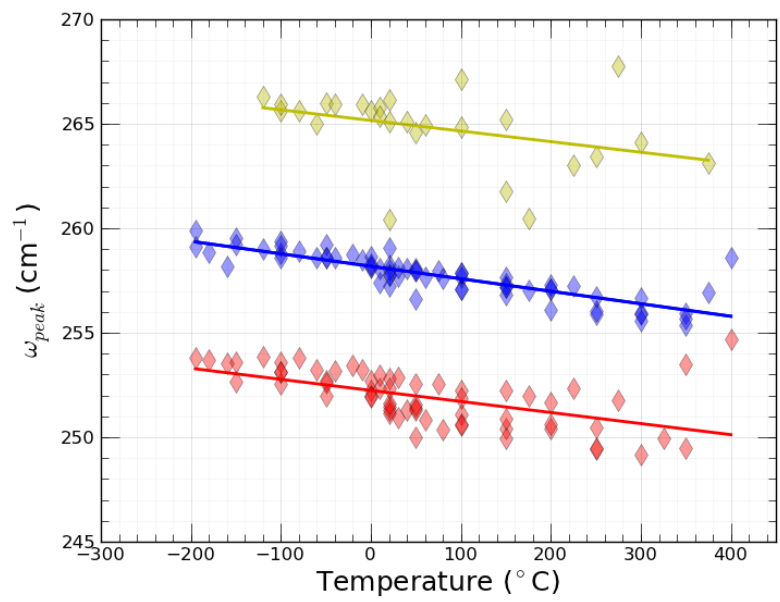

(c) $250 \mathrm{~cm}^{-1}$ composite

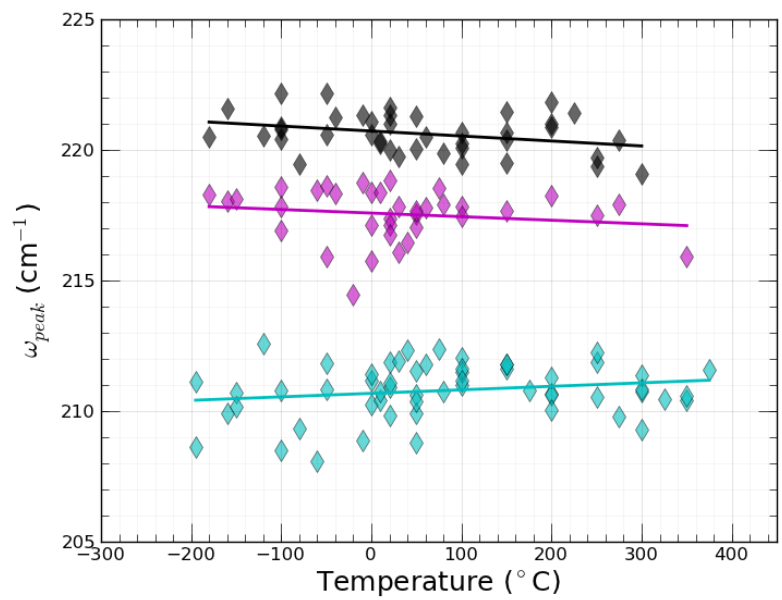

(b) $220 \mathrm{~cm}^{-1}$ composite

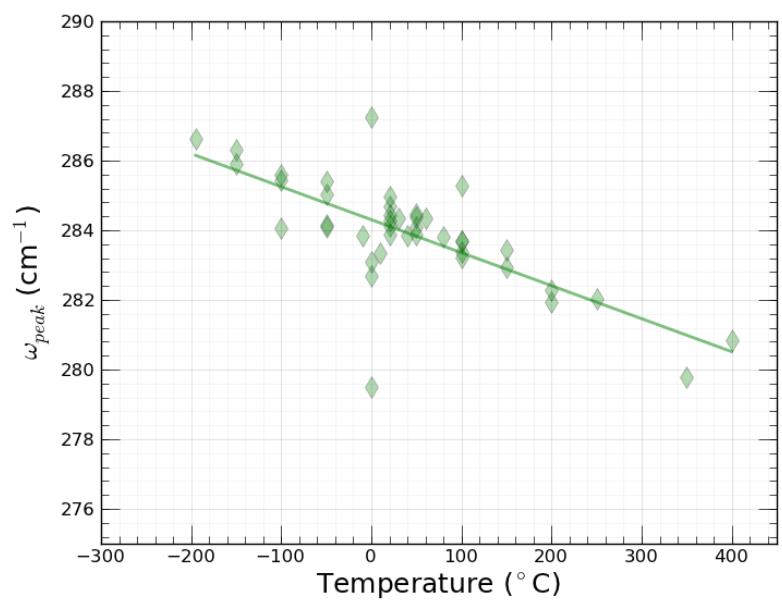

(d) $284 \mathrm{~cm}^{-1}$ peak

Figure 6.2: Temperature dependence of the RBM peak frequencies.

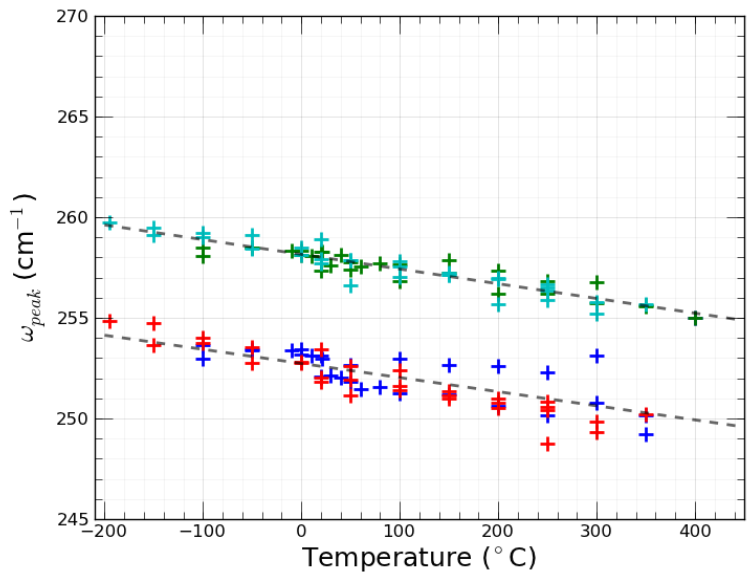

(a) Analysis of $250 \mathrm{~cm}^{-1}$ only

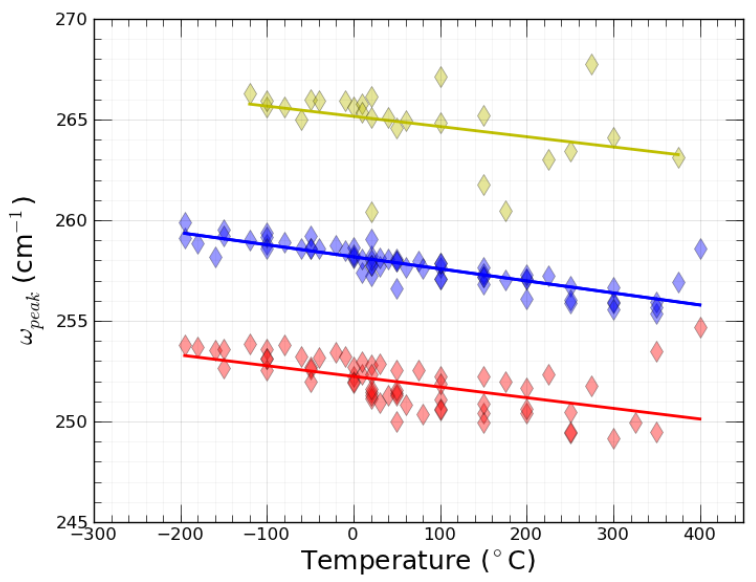

(b) Full spectrum fitting

Figure 6.3: Frequency softening of the $250 \mathrm{~cm}^{-1}$ composite using two methods. 


\begin{tabular}{|c|c|c|c|c|c|}
\hline$\omega_{\text {peak }}$ & $(n, m)$ & $\chi\left(\mathrm{cm}^{-1} / K\right)$ & $\alpha_{R B M}\left(K^{-1}\right)$ & $\alpha_{l i t}\left(K^{-1}\right) 1$ & Source \\
\hline \hline 252 & $(10,3)$ & -0.0050 & $-2.10 \times 10^{-5}$ & - & - \\
\hline 256 & $(11,1)$ & -0.00598 & $-2.31 \times 10^{-5}$ & - & - \\
\hline 264 & $(7,6)$ & -0.00507 & $-1.91 \times 10^{-5}$ & $-3.41 \times 10^{-5}$ & Raravikar et al. \\
\hline 284 & $(7,5)$ & -0.00948 & $-3.33 \times 10^{-5}$ & $-1.0 \times 10^{-5}$ & Zhang et al. \\
\hline \hline
\end{tabular}

Table 6.1: Softening coefficients and comparisons with available literature values.

provided by Raravikar et al. [112] with some citing an absence of phonon decay mechanisms [116] for the studied temperature range. Anharmonicity is considered insignificant since the maximum experimental temperatures are usually around $800 \mathrm{~K}$; much smaller than the Debye temperature (2500 K in graphene).

Raravikar et al. specfically studied the temperature dependence of RBM frequencies from bundled SWNTs. Using both simulation and experiment, they concluded that changes to the RBM frequency result from changes to the inter- and intra-tube force constants with temperature. The pivotal point in their argument favouring the new mechanism was the discrepancy between the predicted $d \omega_{R B M} / d T$ from their simulations with experimental values of thermal expansion; the simulated downshift requiring a thermal expansion coefficient of $7 \times 10^{-6}$ $\mathrm{K}^{-1}$. Disagreement between this value and the experimental value of $-1.5 \times 10^{-6} \mathrm{~K}^{-1}$ was argued as compelling evidence for a different mechanism of bond expansion. The authors' make no comment on the possibility of phonon decay processes giving rise to a frequency shift with temperature. Phonon decay processes operate in the regime of low phonon amplitudes. All temperatures below the Debye temperature are considered part of this regime. A possible lower limit for observing anharmonic effects can be obtained from the $\mathrm{G}^{+}$optical phonon lifetime (of a HiPCO film ) which remains constant till $\sim 100 \mathrm{~K}$ [118]. The minimum temperature from most of the studies listed previously [109, 119, 120] - with the exception of Karachevtsev et al. [111 - is $300 \mathrm{~K}$. Yet, these studies rely on the model of Raravikar et al. to explain their results of the RBM temperature dependence without appropriate consideration of phonon decay processes. Temperature dependence studies of the $\mathrm{G}^{+}$frequency in both graphene and SWNTs [121, 122, 123] all suggest three phonon decay as the dominant mechanism.

We suggest additional studies to resolve this question of the mechanism behind anharmonicity of the RBMs. The model of Raravikar et al. implies that changing force constants dominate the anharmonicity. On the other hand, phonon decay mechanisms can be observed at low temperatures in other Raman modes. Perhaps a theoretical study of anharmonicity due to phonon decay processes - similar to a recent study on $\mathrm{TiO}_{2}$ [124] - can clarify the issue.

Table 6.1 shows both the softening $\left(\chi_{R B M}\right)$ and normalised softening coefficients $\left(\alpha_{R B M}\right)$ for the nanotubes with a strong linear change in frequency with temperatue $\left(\omega_{\text {peak }}>250 \mathrm{~cm}^{-1}\right)$. The softening coefficient is the slope of the linear fit which is then normalised to the peak frequency at $0{ }^{\circ} \mathrm{C}$ (the y intercept from the linear fits shown in Figure 6.2). The values of 
$\alpha_{R B M}$ are clustered about $2 \times 10^{-2}$ and comparable to the values for individual SWNTs from Zhang et al. The 264 and $284 \mathrm{~cm}^{-1}$ peaks (the only two that can be directly matched according to $(n, m)$ indices) are not sufficiently different to suggest a change in softening for bundled SWNTs. Diameter dependence of $\alpha_{R B M}$ only exists for tubes with diameter greater than 1.4 $\mathrm{nm}$ [109]. There is no diameter dependence of $\alpha_{R B M}$ for nanotubes with diameter around $1 \mathrm{~nm}$ (like those from the HiPCO process).

\subsubsection{Peak linewidth}

Like the previous section, both the $250 \mathrm{~cm}^{-1}$ composite and full spectrum fitting routines were used to obtain the temperature dependence of the linewidth. The similarity between the linewidths obtained from the two methods is verified in Figure 6.4. In some cases, the lower resolution fitting of the $250 \mathrm{~cm}^{-1}$ composite leads to poor estimation of $\Gamma$. Figure $6.4 \mathrm{~b}$ shows one such dataset where the higher resolution fitting routine identifies a clear trend of linewidth with temperature while the single composite fitting routine shows no such trend. Only the high resolution fitting data is used for the following discussion on scattering mechanisms and the optical phonon lifetime.

The increasing linewidth for temperatures above $-190{ }^{\circ} \mathrm{C}(\sim 80 \mathrm{~K})$ contradicts an earlier study of Raman linewidths from SWNT bundles [113. Linewidths of the three, single resonance Raman modes (RBM, G-mode and G-mode) were found to only increase perceptibly above $400 \mathrm{~K}$. The apparent contradiction is due to a lack of deconvolution. The authors' chose not to deconvolute the composite. Thus, the RBM components within the composite varied with temperature in a way that composite linewidth was either unaffected, or changed slower than the components. The fitting routines used in this study avoid these issues by fitting the components rather than the composite. Here, the fitted linewidths represent the general behaviour of a single RBM. The word 'general' is used due to the fitting criterion that fits one linewidth for the entire RBM spectrum rather than fitting a different linewidth for every RBM.

The linear trend of the linewidth with temperature (Figure 6.4a suggests a three phonon decay process. But fitting the data to the symmetric version of the model (Eq. 2.11; two daughter phonons of equal magnitude and opposite momenta) produces a poor fit. The fit over-estimates the temperature dependence and under-estimates the linewidth as $T \rightarrow 0 \mathrm{~K}$. The poor quality of the fit suggests either (i) asymmetric decay (daughter phonons of different magnitude) [118] or, (ii) the combination of temperature-independent and dependent components of the linewidth [114.

There is a high probability of asymmetric decay channels in materials with a limited number of low frequency phonon brances e.g. graphene. In graphene, the decay channels can be determined from the phonon dispersion [125]. This is not the case for SWNTs; the number 

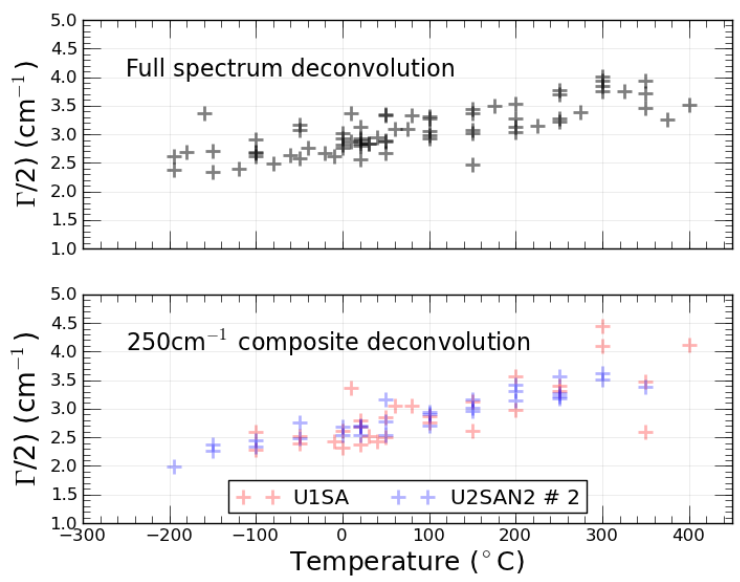

(a) Analysis of $250 \mathrm{~cm}^{-1}$ only

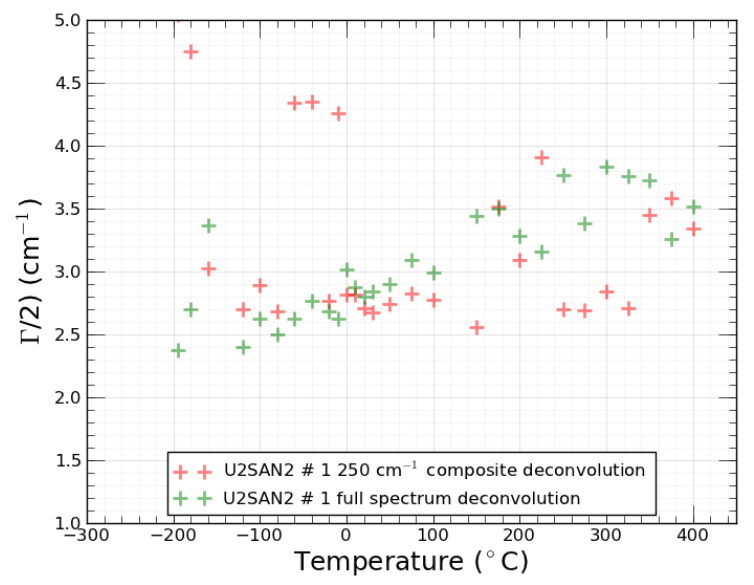

(b) Full spectrum fitting

Figure 6.4: Temperature dependence of linewidth.

of phonon branches in a simple $(n, n)$ tube is far greater than that of graphene. However, there are no theoretical predictions of anharmonic decay channels for SWNTs. Where applicable - such as the shared G-mode - the temperature dependence of optical phonon lifetimes in SWNTs is modelled using the phonon decay channels determined in graphene [125]. Since no such connection exists for the RBMs, modelling the data in terms of asymmetric decay channels is questionable. In fact, fitting the data using this model gives unphysical parameters; the daughter phonons have a greater frequency than the parent RBM phonon.

$$
\Gamma(T)=\gamma+\Gamma_{0} \quad 1+\frac{2}{e^{\hbar \omega / 2 k_{B} T}-1}
$$

Iliev et al. [114] showed that the RBM linewidth can be fitted with a combination of temperature-dependent and independent terms. This model appends an additional fitting parameter, $\gamma$ - the temperature-independent term - to the Klemens model as shown in Eq. 6.1. In the Klemens model, both the slope of $d \Gamma / d T$ and the limiting linewidth as $T \rightarrow 0 \mathrm{~K}$ are given by the $\Gamma_{0}$ parameter. In the modified Klemens model, these two functions are controlled separately. The limiting linewidth is now given by $\gamma$ and $\Gamma_{0}$ controls the slope. Larger values of $\Gamma_{0}$ give a higher rate of increase for the temperature-dependent linewidth (Figure 6.5a). Figure 6.5b shows the data from Figure 6.4a fitted to Eq. 6.1. The fit gives $\gamma$ and $\Gamma_{0}$ as 4.6 and $0.41 \mathrm{~cm}^{-1}$ respectively. The temperature-independent linewidth gives the RBM phonon lifetime as $1.2 \mathrm{ps}$. This value is comparable to the RBM lifetime determined by Jorio et al. [113] and even the lifetime of the G-mode phonon. 


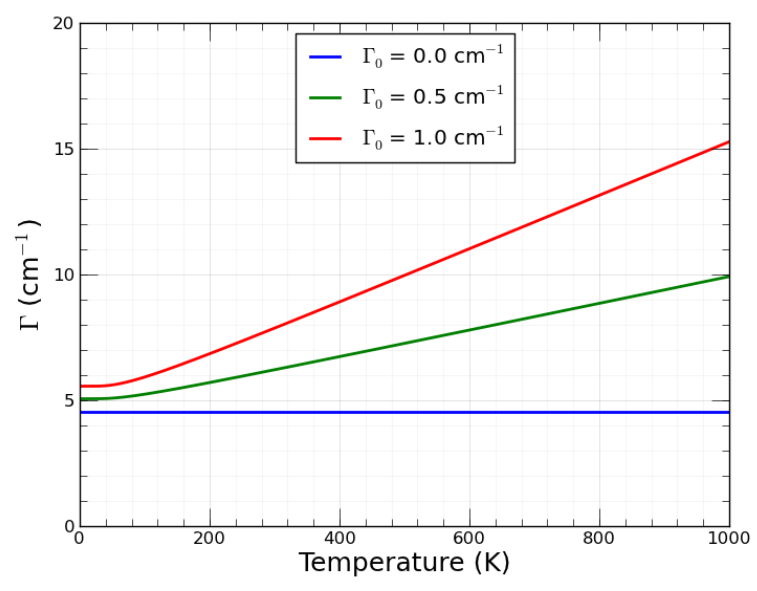

(a) Effect of $\Gamma_{0}$

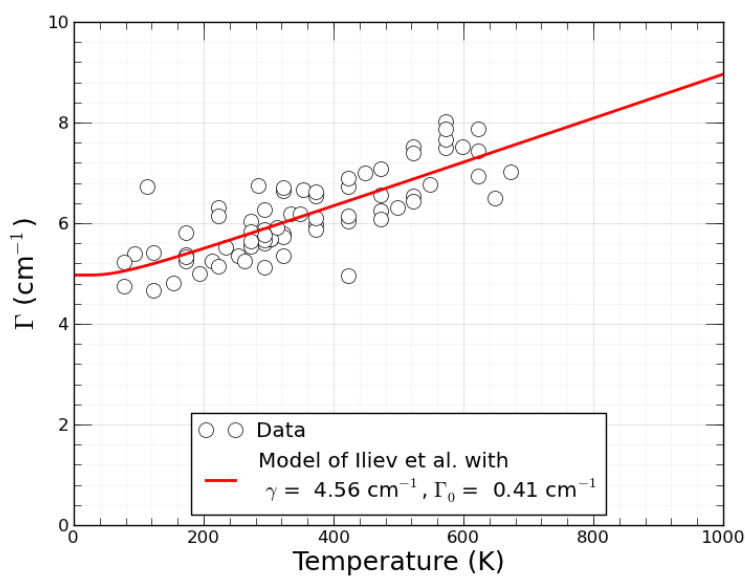

(b) Model of Iliev et al.

Figure 6.5: Fitting linewidth data.

\subsubsection{Peak intensity}

The RBM spectrum at $1.96 \mathrm{eV}$ is comprised of three main composites at 200, 220 and 250 $\mathrm{cm}-1$. Of the three, the component RBMs of the 220 and $250 \mathrm{~cm}^{-1}$ composites are situated on the high and low energy side of $E_{\text {laser }}$ respectively. The relative size of the composites indicates the degree of both bundling and temperature. As bundling (temperature) increases, the resonance window broadens and its center moves to a higher energy (Chapter 5.2.2). The following section quantifies the relationship between the composite size and temperature. The results of this analysis are used as a qualitative indicator of local temperature in the following section on local heating (Section 6.3).

The 'size' of the composite is a qualitative term. The quantitative version is the signal. In Chapter 2.1.1, the Raman signal was defined as equivalent to the number of Raman photons; a quantity that can be associated with the integrated intensity of the Raman mode. In this case, the Raman signal of a composite containing several component Raman modes is required. So, integrated intensities are calculated based on a frequency range i.e., if the peak frequency of a component RBM falls within the range for a given composite, it is included in the calculation of integrated intensity for that composite. The frequency ranges for calculating the Raman signal of the composites are idenitified on the basis of nanotube families (family indices are given in brackets): $200(15,0), 220(13,1)$ and $250 \mathrm{~cm}^{-1}(11,1)$. The ranges are: $170-205 \mathrm{~cm}^{-1}$ for $(15,0) ; 205-235 \mathrm{~cm}^{-1}$ for $(13,1)$ and, $235-275 \mathrm{~cm}^{-1}$ for $(11,1)$. The integrated intensity of the composite is the the sum of the integrated intensities of the component RBMs within the specified frequency range. Since the total signal from a given spot is not always constant, the integrated intensity for each composite is normalised by the total integrated intensity of the full RBM spectrum.

The unambiguous SWNTs associated with the above frequency ranges are listed in Table 5.1 


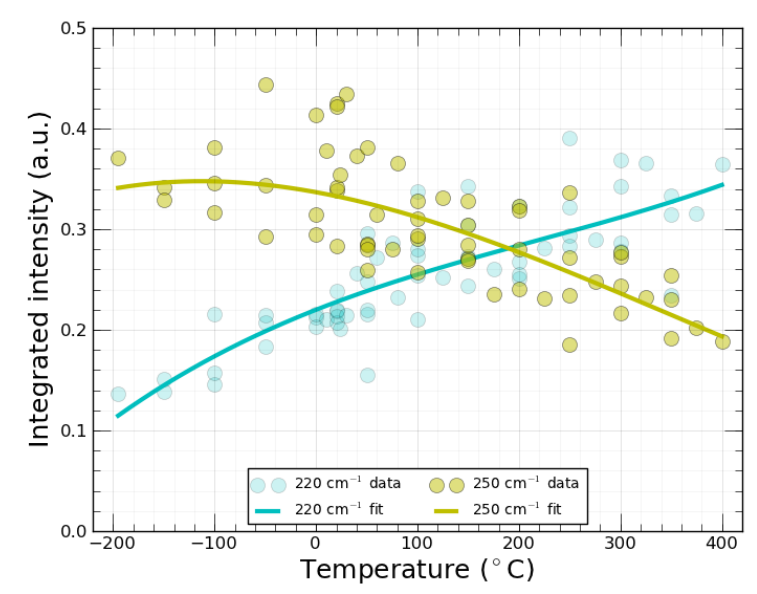

(a) Composite signal

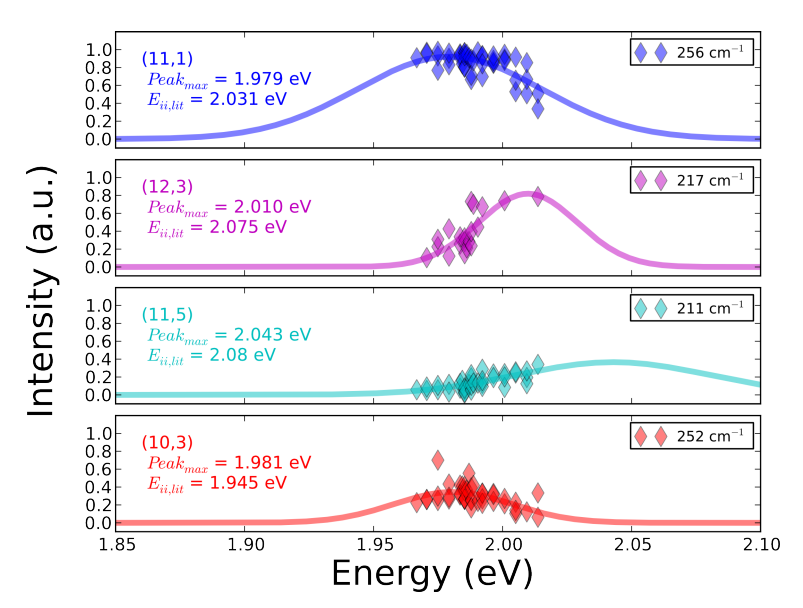

(b) Peak intensity

Figure 6.6: Temperature dependence of the 220 and $250 \mathrm{~cm}^{-1}$ RBM composites (a) and some of peaks within (b).

and the full list can be found in [94]. While the majority of nanotubes within a composite belong to the same family, there are some notable exceptions. The 264 and $205 \mathrm{~cm}^{-1} \mathrm{RBMs}$ in particular. In the former, the nanotube is considered part of the $250 \mathrm{~cm}^{-1}$ composite since there are no other resonant tubes from its own branch. The $205 \mathrm{~cm}^{-1}$ peak, however, is associated with the $200 \mathrm{~cm}^{-1}$ composite rather than the $220 \mathrm{~cm}^{-1}$ because it cannot be unambiguously separated from the $204 \mathrm{~cm}^{-1}$ peak that belongs to the $(15,0)$ family.

Figure 6.6a shows the temperature dependence for the 220 and $250 \mathrm{~cm}^{-1}$ composites. Trendlines are calculated using a smoothing spline fit to the data. The trend for the $250 \mathrm{~cm}^{-1}$ composite begins to decrease steadily after $0{ }^{\circ} \mathrm{C}$ while the trend for the $220 \mathrm{~cm}^{-1}$ peak monotonically increases from the lowest measured temperature. The two trendlines cross at 200 ${ }^{\circ} \mathrm{C}$. At this temperature, the relative signal from each composite contributes $25 \%$ to the total Raman signal. A similar cross-over is observed in the data of Uchida et al. [108] at $500{ }^{\circ} \mathrm{C}$.

The redshifted cross-over temperature for the films in this study are a consequence of the higher degree of bundling. The $220 \mathrm{~cm}^{-1}$ composite (also called the 'roping' composite since the component RBMs have $E_{i i}$ greater than the laser energy) moves into resonance at a lower temperature. However, the mechanism behind its early appearance into resonance is still unclear. Either the $E_{i i}$ are already red-shifted and/or, they have a faster rate of decrease with temperature in bundled samples. The $E_{i i}$ are known to redshift in bundles [93] but the effect of bundling can be convoluted with an increase in local temperature due to laser heating [76]. The remainder of this section uses the temperature dependence of the observed RBM intensity to calculate the rate of change of $E_{i i}$ with temperature.

The temperature dependence of the intensity for four RBMs - two from each of the two main composites - are shown in Figure 6.6b. In this figure, the $\mathrm{x}$-axis represents temperature 

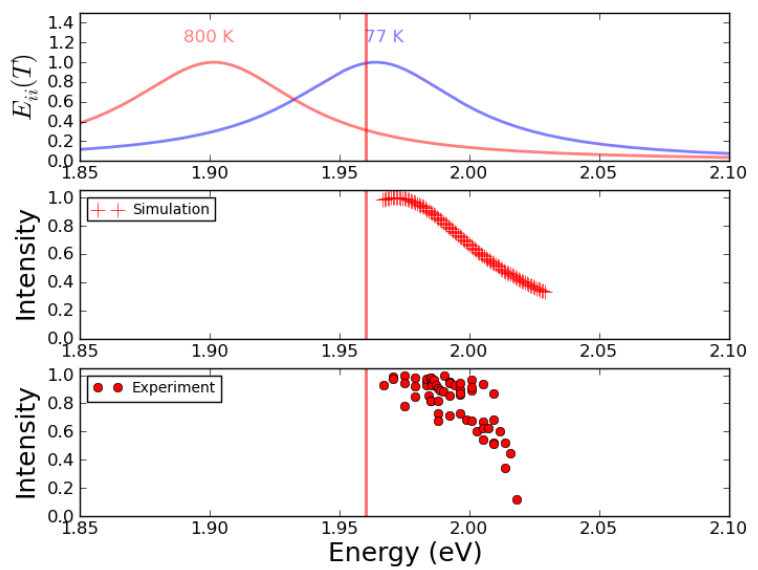

(a) $256 \mathrm{~cm}^{-1}$ peak

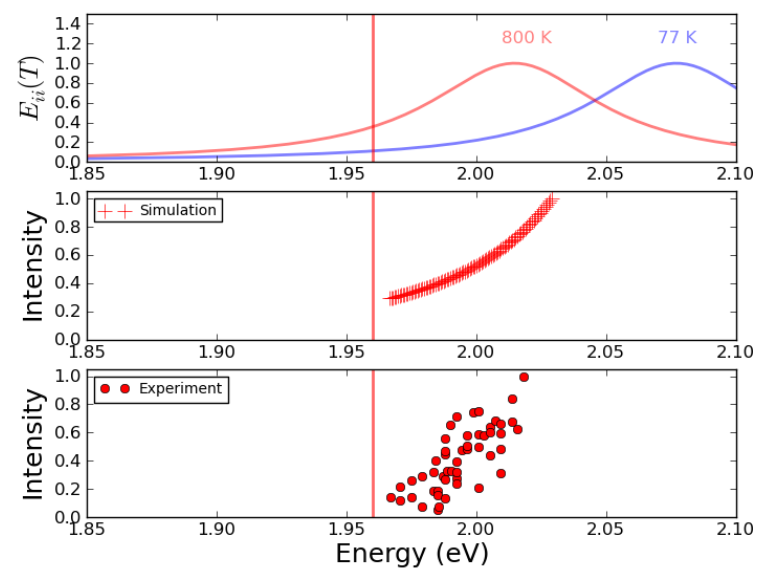

(b) $211 \mathrm{~cm}^{-1}$ peak

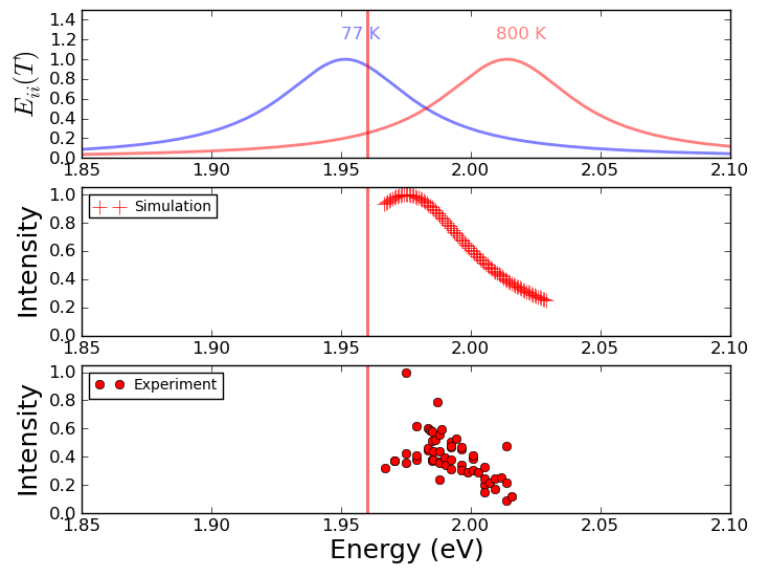

(c) $252 \mathrm{~cm}^{-1}$ peak

Figure 6.7: Temperature dependence of the RBM intensity for three peaks within the 220 and $250 \mathrm{~cm}^{1-}$ composites. The top subplot presents the $E_{i i}$ at 77 (blue curve) and $800 \mathrm{~K}$ (red curve). The middle and bottom subplots show the simulated and observed RBM intensities respectively. 
but as $k_{B} T+E_{\text {laser }}$. This unusual transformation of temperature is done so that the $E_{i i}$ and the RBM intensity can be compared on the same scale. The temperature dependence of the RBM intensity can be simulated using a simple model that only relies upon the change in $E_{i i}$ with temperature [76, 98]. The temperature-dependent RBM intensity was simulated for three of the four RBMs shown in Figure 6.6b. The $217 \mathrm{~cm}^{-1}$ peak was not simulated because the available data points were insufficient for determining a trend. The basic steps of the simulation are as follows:

1. The $E_{i i}$ for the three nanotubes were taken from [94]. The shape of $E_{i i}$ was modelled using Eq. 5.1 with $\gamma$ of $40 \mathrm{meV}$.

2. The intensity of the modelled curved at the laser energy was recorded.

3. $E_{i i}$ was then red- or blueshifted by $\mathrm{k}_{B} T$. The intensity of the curve at the laser energy was again recorded.

4. The above step was carried out for temperatures between $77 \mathrm{~K}$ and $800 \mathrm{~K}$.

Minor adjustments were made to some of the parameters until a visual match to the data was obtained. The simulation results for three RBMs are shown in Figure 6.7. All three figures show that the simulated data are a strong (albeit qualitative) match to the observed data. The minor adjustments were a $60 \mathrm{meV}$ downshift to the $E_{i i}$ for the $256 \mathrm{~cm}^{-1}$ peak, and a blueshift in $E_{i i}$ with temperature for the $252 \mathrm{~cm}^{-1}$ peak. The manual redshift of the $E_{i i}$ for the 256 $\mathrm{cm}^{-1}$ peak strongly suggests that $E_{i i}$ are altered in bundle. While highly probable (parameters pertaining to the $256 \mathrm{~cm}^{-1}$ peak are the most accurate as it is best resolved peak of the RBM spectrum at $1.96 \mathrm{eV}$ ), less noisy data is required for a conclusive assertion that the $E_{i i}$ is altered upon bundling. The best solution is a separate study of $E_{i i}$ determined from the RBM intensity as a function of laser energy. The blueshifted $E_{i i}$ for the $252 \mathrm{~cm}^{-1}$ agrees with the findings of Fantini et al. [76]: $E_{i i}$ from SWNTs with $\nu=+2$ (Chapter 5.2.2) blueshift with temperature while the $E_{i i}$ from all other SWNTs are redshifted. The $252 \mathrm{~cm}^{-1}$ peak has the index $(10,3)$ and applying the formula for $\nu((2 n+m) \bmod 3))$ gives +2 .

\subsection{Local heating}

In this final section, we examine the effects of local heating on the RBMs. Local heating in SWNT networks can occur if the probed area is physically disconnected from the rest of the network. When the probed area is a small clump of material, only heat conduction via the network is suppressed; conduction to the substrate heat sink is possible. Both heat conduction pathways are suppressed when the clump is large. A sample where prolonged deposition of wet solution created a non-conducting network with a non-uniform morphology was used in this study. The effects of local heating are studied in two ways: single spot power dependence and spatial mapping. The characteristics of local heating determined from the single region are 
compared to the same quantities obtained from the spatial map.

\subsubsection{Data collection}

Local heating effects for a single region of bundled SWNTs were determined from a power dependence study. An axial dependence of the RBM spectrum at a given spot is equivalent to a sensitive power dependence measurement for the LabRam which only has coarsely graded neutral density filters for altering the laser power. RBM spectra were collected every micron up to $\pm 15 \mu \mathrm{m}$ from the focal plane. Spatial variations in local heating were measured by collecting Raman spatial maps at two powers: full power $\left(P_{0}\right)$ and low power (setting D1 or $\mathrm{P}_{0} / 10$ ). Data acquisition was carried out such that the first spatial map was at full power and the second at low power.

Given such a large volume of data, only the $250 \mathrm{~cm}^{-1}$ composite was studied in detail. The composite was deconvoluted as described in Section 5.4.2, The following parameters were studied: linewidths, peak frequencies and the relative signal intensities of the 250 and $220 \mathrm{~cm}^{-1}$ composites. The linewidths and frequencies were extracted from the deconvolution. Relative signal intensity was calculated slightly differently to the method outlined in Section 6.2.4 where the integrated intensity was calculated from the sum of the component integrated intensities. In this analysis, only the $250 \mathrm{~cm}^{-1}$ composite is deconvoluted and the components for the remainder of the spectrum are unknown. Hence, the integrated intensity for a composite is calculated from the spectral data. The frequency range of each composite remains the same (170 - $205 \mathrm{~cm}^{-1}$ for $(15,0) ; 205-235 \mathrm{~cm}^{-1}$ for $(13,1)$ and, $235-275 \mathrm{~cm}^{-1}$ for $\left.(11,1)\right)$.

\subsubsection{Characteristics of local heating}

The three characteristics of the RBM spectrum that vary with temperature are the peak frequency, linewidth and the relative signal from composites close to $\left(250 \mathrm{~cm}^{-1}\right)$ and higher in energy $\left(220 \mathrm{~cm}^{-1}\right)$ from the laser line. The established variation of these characteristics with temperature makes them semi-quantitative metrics of temperature when the heat source cannot be monitored externally.

Figures $6.8 \mathrm{a}$, $6.8 \mathrm{~b}$ and $6.8 \mathrm{c}$ plot the $256 \mathrm{~cm}^{-1}$ peak frequency, linewidth and relative signal from the two composites as a function of axial displacement (effective laser power). The window of axial displacement where major changes are seen in all three parameters is $\pm 5 \mu \mathrm{m}$ from the focal plane. Using a irradiation radius of $1 \mu \mathrm{m}$ and the full power at the focal plane as 6.7 $\mathrm{mW}$, the effective power at the onset of local heating (axial displacement of $\pm 5 \mu \mathrm{m}$ ) is $3 \mathrm{~mW}$. Within the heating window, the 'inverse- $V$ ' and ' $V$ ' shapes of the linewidth and peak frequency show that the two parameters are linearly correlated. The same linear correlation is identified 


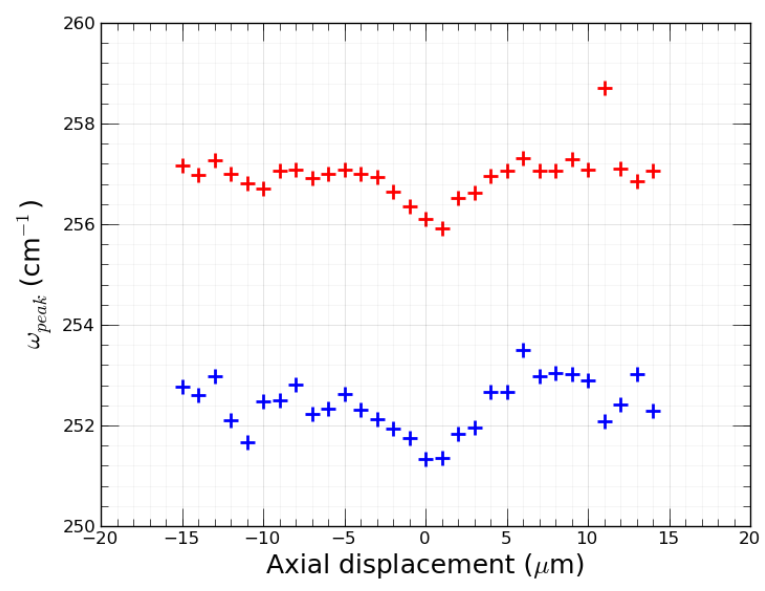

(a) Peak frequency

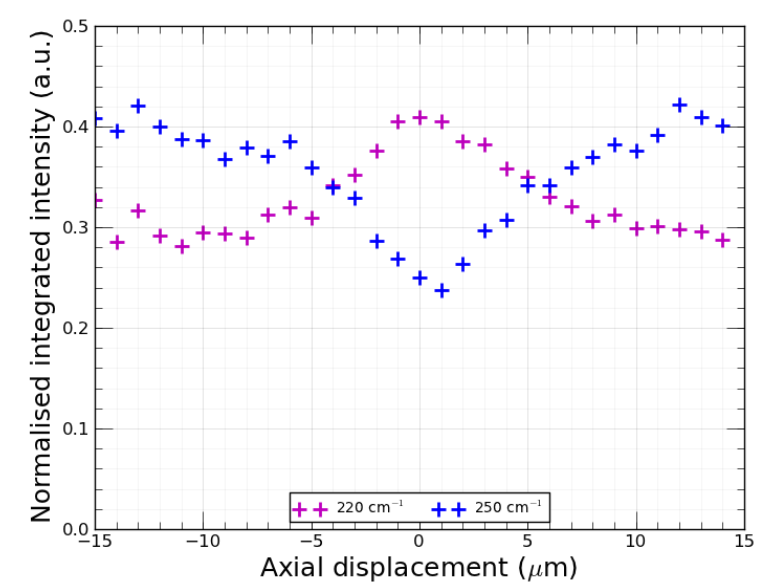

(c) Composite signal

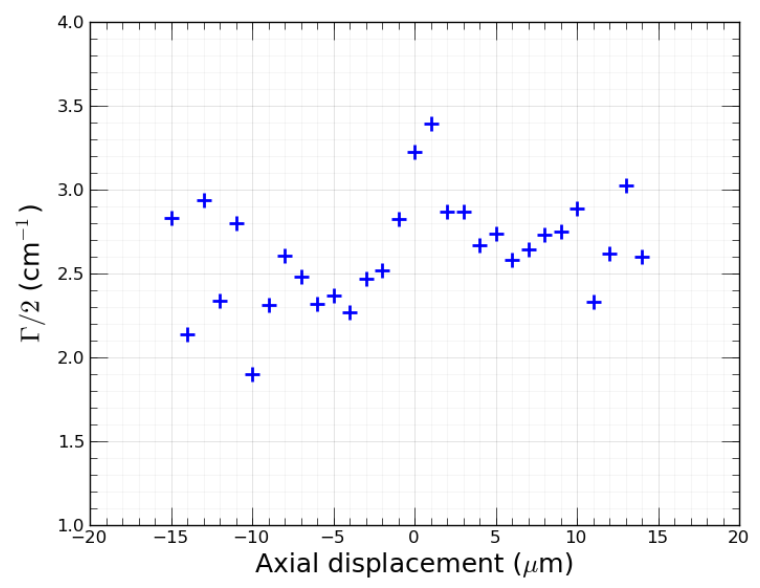

(b) Linewidth

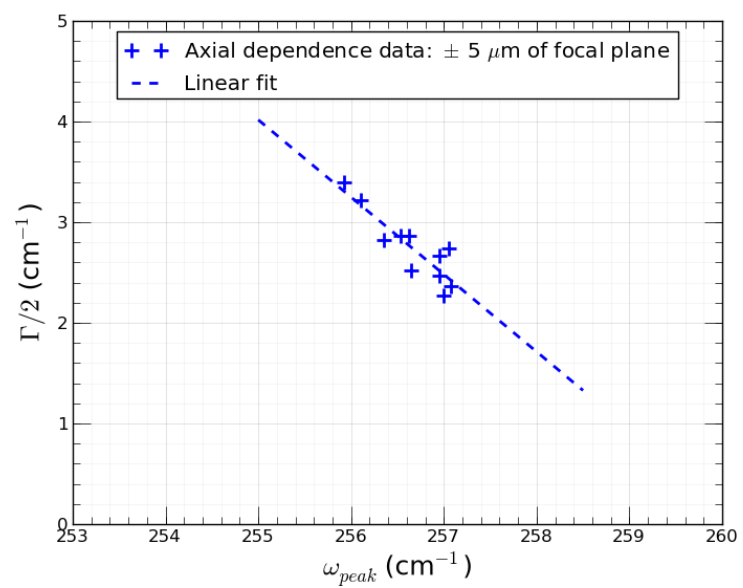

(d) Frequency vs. linewidth

Figure 6.8: Axial dependence of various parameters from a bundled region of a thick SWNT film.

for the 220 and $250 \mathrm{~cm}^{-1}$ composites. The maximum temperature rise occurs at the focal plane and can be estimated as $100{ }^{\circ} \mathrm{C}$ using $\omega_{\text {peak }}(T)$ for the $256 \mathrm{~cm}^{-1}$ tube (Figure 6.2) and $150{ }^{\circ} \mathrm{C}$ using $\Gamma(T)$ (Figure 6.5b). Similar predictions of the local temperature cannot be made with the composite signal since the calculation of integrated intensity differs from Section 6.2.4.

Figure 6.8d shows the linewidth plotted against peak frequency for axial displacements between $-5 \mu \mathrm{m}$ and $5 \mu \mathrm{m}$. The correlation between the two parameters can be fitted with a straight line as discussed in the above paragraph. Hence, $\omega_{\text {peak }}$ still remains a reliable proxy for temperature. The linewidth can also be used but lacks accuracy for low signal datasets. The peak frequency of the $256 \mathrm{~cm}^{-1}$ peak on the other hand, is always well resolved. At this point, it is worth reiterating that local heating is only seen in the U2SAN2 sample since it contains isolated, thick clumps of SWNTs. Figure 6.9 compares axial dependence of the 256 $\mathrm{cm}^{-1}$ peak between a thin sample and the thick sample. The thin sample, D2S, has a higher $\omega_{\text {peak }}$ (discussed in Chapter 5.5) but shows no change near the focal plane. 


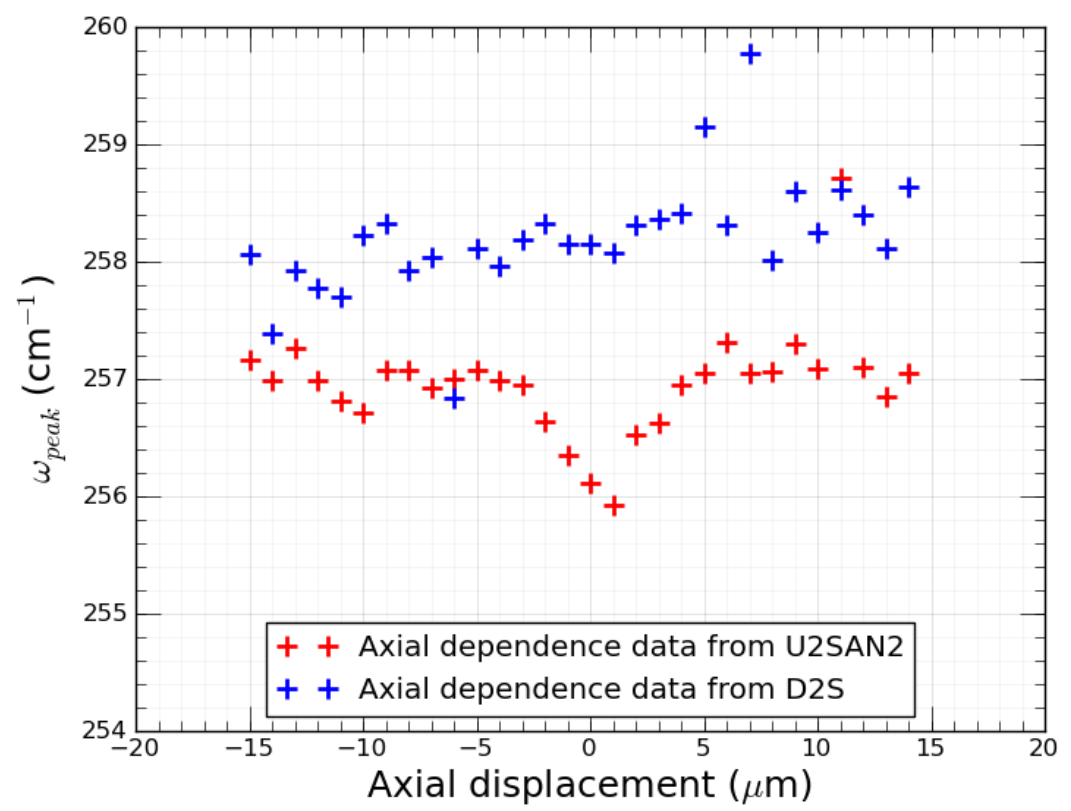

Figure 6.9: Axial dependence from a bundled region of a thick SWNT film vs. thin film.

\subsubsection{Heating effects from spatial mapping}

Establishing the characteristics of local heating required a simple experiment where the sample region was kept constant and the power varied. A more complex experiment of spatial mapping also gives the same results. The variation of SWNT density and laser power as the laser tracks across the sample also leads to local effects; effects that mirror the characteristics identified in Section 6.3.2. A spatial variation in local heating draws a picture of sample inhomogeneity e.g. large variations in SWNT density. In the absence of visual evidence, a spatial map could qualitatively establish the degree of homogeneity within a sample. The greater the extent of local heating, the greater the inhomogeneity.

Figure 6.10 shows a matrix of frequency histograms for the annealed sample at high and low powers. At high power, the components of the $250 \mathrm{~cm}^{-1}$ composite are increased to include the $242 \mathrm{~cm}^{-1}$ and $277 \mathrm{~cm}^{-1}$ peaks. The appearance of these two peaks indicates a broadening of the resonance window due to a shift and broadening of the underlying electronic transitions (Table 5.1). Both these nanotubes have $E_{i i}$ considerably higher than the laser energy [94]. At high power, the peak frequencies are redshifted. The maxima of the gaussians fitted to the histogram data are decreased by $1 \mathrm{~cm}^{-1}$ at higher powers. A connected feature (though more subtle) is the dispersion of frequencies within the histogram. The dispersion of frequencies for the two main peaks - 252 and $256 \mathrm{~cm}^{-1}$ - is reduced by half at low power. Both the redshift in the average peak frequency and increased resonance window point to an increase in local temperature. 


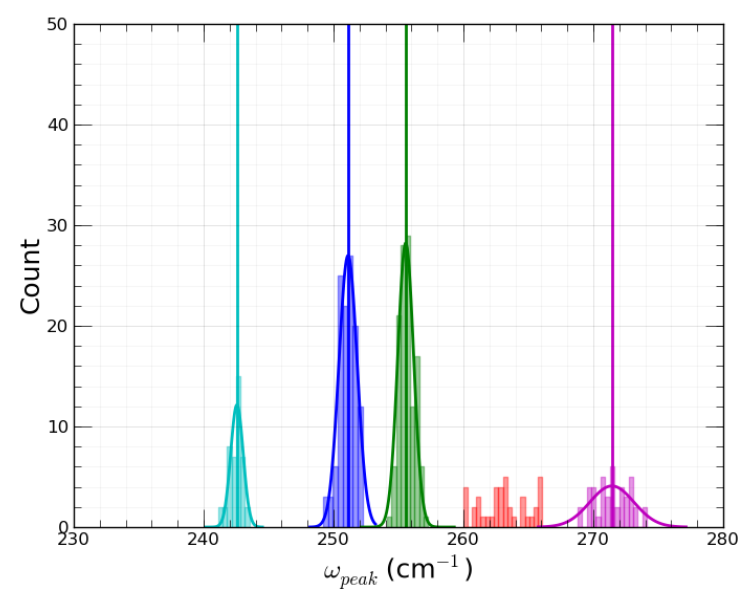

(a) Full power $\left(\mathrm{P}_{0}\right)$

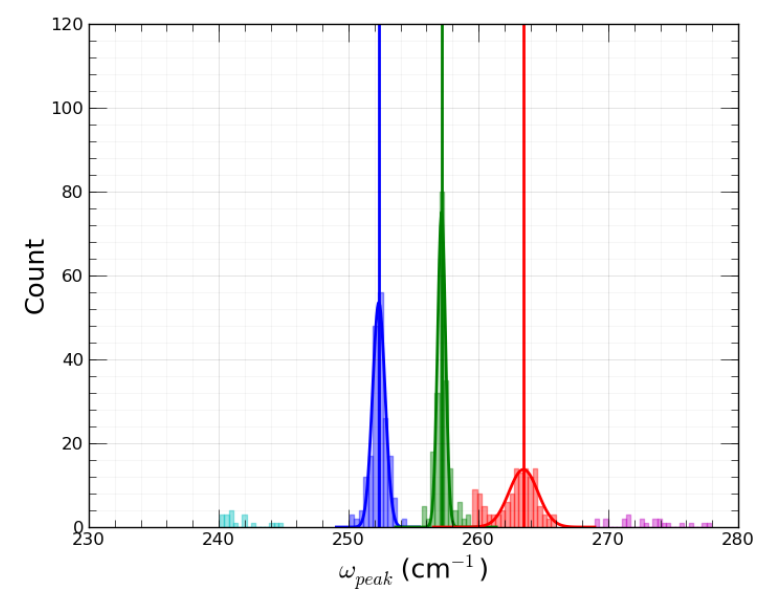

(b) Low power $\left(P_{0} / 10\right)$

Figure 6.10: Power dependence of the RBM frequency histogram that follows the transformation of a solvent covered sample to one without.

Thus, we can conclude that variable density of SWNTs in the U2SAN2 sample induces a range of local temperatures during spatial mapping. As outlined in Sections 8.2 and 6.3 .2 , the RBM spectrum from regions heated above ambient exhibit three changes: decreased $\omega_{R B M}$, increased $\Gamma$ and larger (lower) signal from $220(250) \mathrm{cm}^{-1}$ composites. Figure 6.11 shows that these effects are also observed for the spatial maps. The linear correlation between the linewidth and frequency is similar to local heating data from a single region (Figure 6.8d).

\subsection{Conclusions}

We have investigated the temperature dependence of the RBM spectrum from pristine HiPCO thin films using the resolution deconvolution methodology. The RBM peak position decrease linearly with increasing temperature with a softening coefficient similar to that of individual SWNTs. We find strong evidence that the change in RBM intensity as a function of global temperature follows from the variation of $E_{i i}(T)$. For semiconducting tubes with $\nu=+1$ and metallic nanotubes, $E_{i i}$ redshifts with temperature. For semiconducting tubes with $\nu=+2, E_{i i}$ blueshifts with temperature. Both these effects have been observed in local heating experiments, where the laser was tuned across the transitions at different laser powers (local temperature) [76]. Our method has the advantage of identifying a temperature since the temperature of the sample is controlled externally - it is difficult to identify an accurate local temperature from power dependence measurements. Combined with our high resolution fitting strategy, we suggest our method of simulating $d E_{i i} / d T$ can be useful in decoupling bundling-related changes to the $E_{i i}$ from temperature-related changes. With an appropriate set of samples where the sample thickness can be controlled accurately e.g. dielectrophoretic deposition, this single laser 


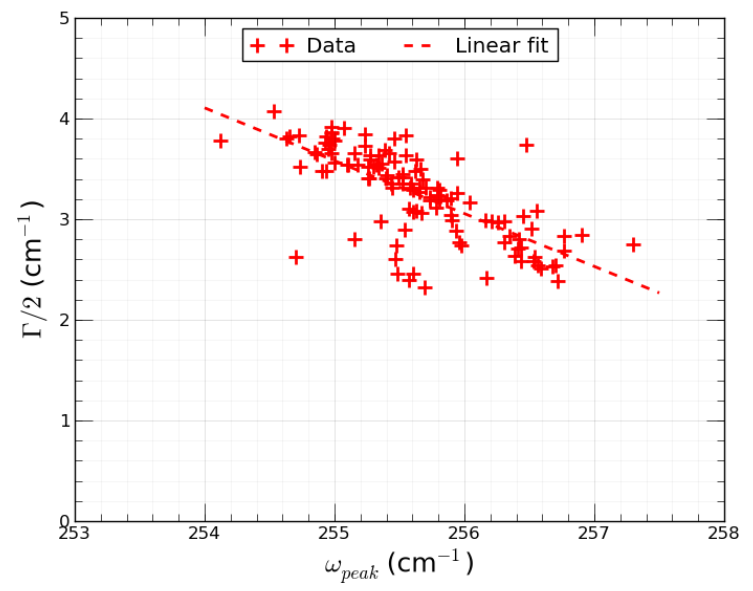

(a) $\Gamma$ vs. $\omega_{\text {peak }}$

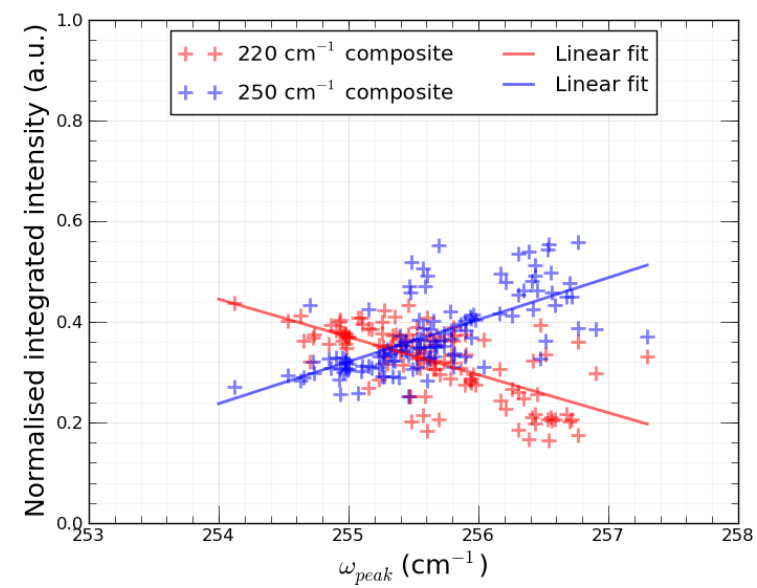

(b) Composite signal

Figure 6.11: Spatial variation of $\Gamma$ vs. $\omega_{\text {peak }}$ (a) and integrated intensity of the 220 and 250 $\mathrm{cm}^{-1}$ composites (b).

method can be used to determine the rate of change in $E_{i i}$ with temperature according to the degree of bundling.

The major changes to the RBM lineshape in pristine thin films with increasing temperature can be summarised as follows: (i) increase in the resonance window, (ii) decrease in $\omega_{R B M}$, (iii) increase in $\Gamma$, and (iv) increase in signal from the 'roping' composite and a decrease in signal from composites closer to the laser energy. These changes are the same for local heating by the laser. From the four different changes, the quickest way to discern local/global effects is from a linear correlation between $\Gamma$ and $\omega_{R B M}$. Our findings from laser heating experiments indicate that the local temperature is strongly correlated to local nanotube density. 


\section{Chapter 7}

\section{Introduction to Cupric Oxide}

\subsection{Introduction}

Cupric oxide $(\mathrm{CuO})$ is a thermodynamically stable oxide of copper at ambient pressure and temperature (Figure 7.1a). This stability is reflected in the $\mathrm{Cu}_{2} \mathrm{O}$ samples are often mixed phase with $\mathrm{CuO}$ [126] due to the disocciation of $\mathrm{Cu}_{2} \mathrm{O} \rightleftharpoons \mathrm{CuO}+\mathrm{O}_{2}$ even at room temperature [127. $\mathrm{CuO}$ crystallises in the monoclinic structure (Figure 7.1b; resulting from a considerable Jahn-Teller distortion of the expected cubic lattice seen in other transition metal monoxides [128]. The monoclinic crystal can be described with the following parameters: $\alpha=\gamma=90^{\circ}$ $; \beta=99.54^{\circ} ; a=4.6837 \AA ; b=3.4226 \stackrel{\AA}{A}$ and $c=5.1288 \AA$ [129]. The optical bandgap is between 1.0 and $1.4 \mathrm{eV}$ [23, 22, 21]. Most experimental studies on bulk $\mathrm{CuO}$ do not allude to the type of optical transition but a recent DFT study was able to demonstrate that this low energy gap is an indirect bandgap [130]. However, this study is only one of its kind and still relatively unsophisticated compared to other DFT methods [131]. There have also been suggestions of a direct bandgap around $3 \mathrm{eV}$ by optical [132] and Raman [133] studies independently but its existence and energy still remains inconclusive. $\mathrm{CuO}$ is p-type semiconductor due to native point defects in the form of $\mathrm{Cu}^{2+}$ vacancies in the lattice. In both $\mathrm{Cu}$-rich and $\mathrm{Cu}$-poor environments, the vacancies introduce an impurity level in the bandgap about $0.2 \mathrm{eV}$ from the valence band edge [130]. As a result, conduction in $\mathrm{CuO}$ proceeds via an activation mechanism with an activation energy corresponding to the impurity level [134].

Despite a bandgap situated near the optimum value of $1.4 \mathrm{eV}$ (for single junction solar cells) and intrinsic p-type conduction, $\mathrm{CuO}$ is not ideal for photovoltaic applications due to the likely indirect nature of the bandgap and poor carrier mobilities. The relatively poor mobility of the hole carriers due to a hole effective mass of $1.87 m_{0}$ [130] (compared to $0.26 m_{0}$ in silicon) means that $\mathrm{CuO}$ would be inadequate for charge separation required for efficient solar cells. Consequently, most technologically-relevant research projects have concentrated on $\mathrm{Cu}_{2} \mathrm{O}$. $\mathrm{Cu}_{2} \mathrm{O}$ with its larger but direct bandgap and high mobilities is considered a far more viable candidate for low-cost solar cells. To date, the two main bodies of $\mathrm{CuO}$ research are focused on (i) using $\mathrm{CuO}$ as a simple model system for understanding superconductivity in the high temperature cuprate 


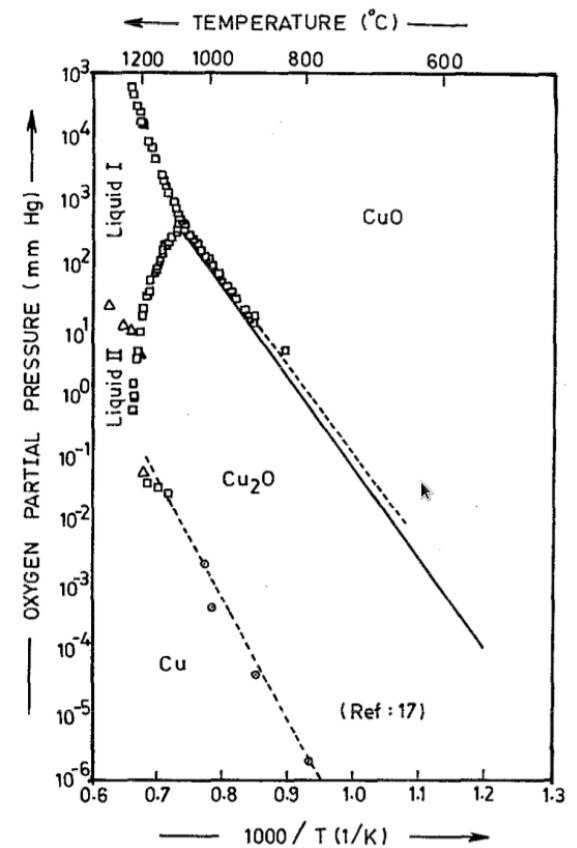

(a) $\mathrm{CuO}$ phase diagram

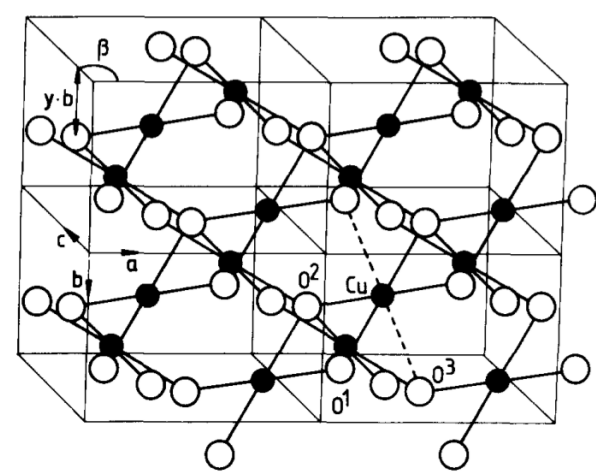

(b) $\mathrm{CuO}$ crystal structure

Figure 7.1: (a) Phase diagram of $\mathrm{CuO} / \mathrm{Cu}_{2} \mathrm{O}$ from [135]. Oxygen partial pressure at ambient is $120 \mathrm{~mm} \mathrm{Hg}$. (b) $\mathrm{CuO}$ crystal structure [136].

systems and, (ii) in the fabrication and characterisation of nanomorphologies from nanowires [137] to nanocrystals [126] to more exotic shapes like nano-shuttles [138], nano-urchins [139] and nano-flowers [140, 141].

The first category contains most of the fundamental studies performed on $\mathrm{CuO}$ in its bulk form. The second is a recent proliferation of $\mathrm{CuO}$ research and particularly emphasises the possibility of novel applications based on the unique structural properties of the different nanosystems. Applications of these structures span a wide range: as field emission sources [142], anode materials in Li-ion batteries [139], conductometric chemical sensors [143] and light switches [141]. The point of commonality between these two fields of research is the use of Raman spectroscopy to probe physical properites like structural transitions and confinement effects. Chrzanowski and Irwin [144] spearheaded the first Raman study of $\mathrm{CuO}$ with a view to compare the temperature dependence of this material to the high $\mathrm{T}_{c}$ cuprates $\mathrm{YBa}_{2} \mathrm{Cu}_{3} \mathrm{O}_{6+y}$ (YBCO) and $\mathrm{La}_{2} \mathrm{CuO}_{4}$ (LCO). Since then the bulk CuO Raman spectrum has been studied for its low temperature properties [144, 133], vibration origins of the various modes using isotope exchange [145], polarisation dependence [146] and pressure dependence [136, 147]. Nanomorphologies of $\mathrm{CuO}$ have been studied with Raman in order to identify phonon confinement effects [148, 149, 150] and electron-phonon coupling [151]. Figure 7.9c shows the summary of changes to the Raman lineshape (in terms of the peak position and linewidth of the $\mathrm{A}_{1 g}$ mode) for spectra collected along the length of a $\mathrm{CuO}$ nanowire [150]. The nanowire in this case has a 'nanoneedle' geometry and decreases in diameter away from the root. The authors summarise that increasing phonon confinement, bought about by the decreasing diameter towards the 
needle tip, results in the red-shifted and broadened Raman peak.

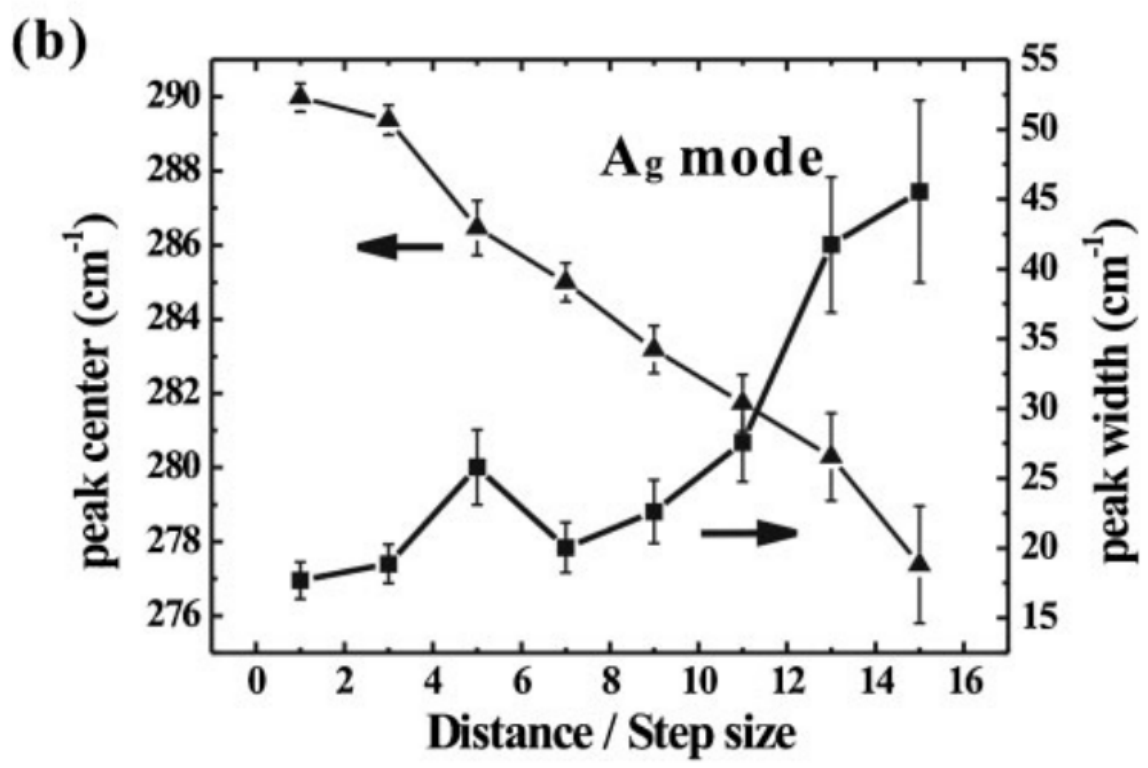

Figure 7.2: Summary of the Raman lineshape obtained from a $\mathrm{CuO}$ nanowire with a "nanoneedle' shape. Figure taken from [150].

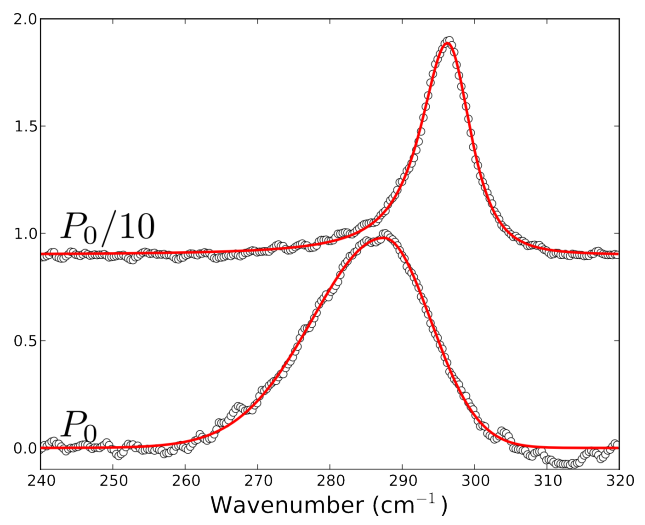

(a) $\mathrm{A}_{1 g}$ mode in a $\mathrm{CuO}$ thin film

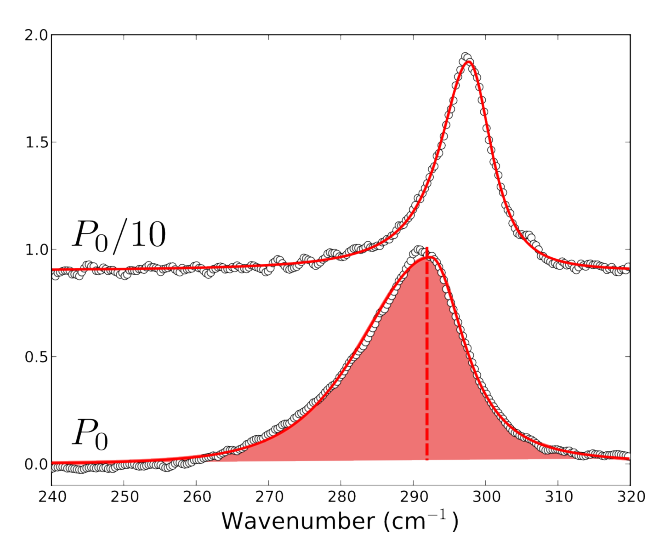

(b) $\mathrm{A}_{1 g}$ mode in a $\mathrm{CuO}$ nanowire forest

Figure 7.3: Raman spectrum from a $\mathrm{CuO}$ thin film and nanowire forest (Figure 7.19) at two powers $\left(\right.$ top $=P_{0} / 10$, bottom $=P_{0}$ ). The full power lineshape for the nanowire forest is highlighted to make the asymmtery clear.

The phenomenological model of phonon confinement effects on the Raman lineshape was first established by Richter et al. [152]. Studies since have refined upon this model depending upon the test nanosystem. An important improvement has been the addition of a term that quantifies the contribution from laser heating to the Raman lineshape. The two types of laser heating: inhomogeneous (spatial temperature distribution) and homogeneous (uniform elevated temperatures), have been well studied in silicon where the former is shown to contribute to the asymmetry of the Raman lineshape [153] while the latter symmetrically broadens the Raman lineshape [154]. In both cases, the authors conclude that phonon confinement effects are mainly present in the strong confinement regime $\left(d \leq r_{b o h r} ; 5 \mathrm{~nm}\right.$ exciton Bohr radius in silicon). The 
inclusion of these additional terms is based on the poor heat transport in nanomaterials due to suppressed thermal conductivities, e.g. the thermal conductivity of silicon decreases by almost a factor of 10 when grain size decreases from $1 \mu \mathrm{m}$ to $100 \mathrm{~nm}$ [155]. Hence, laser heating can be a strong effect for nanomaterials with dimensions both within and above the confinement regime.

The Bohr radius of $\mathrm{CuO}$ can be calculated as $1.7 \mathrm{~nm}$ (details in Section 7.3.2) and the Raman spectrum of any $\mathrm{CuO}$ nanomorphologies with dimensions above this value (such as the sample tested in [150]) most likely exhibit strong local heating by the laser rather than phonon confinement effects. However, high temperature dependence of the $\mathrm{CuO}$ Raman spectrum is limited to one low resolution study that only tracked the effect of temperature on the Raman peak positions. Chapter 8 addresses this very gap in the literature by a detailed investigation into the effects of global and laser heating in $\mathrm{CuO}$. The underlying mechanisms of mode anharmonicity are fleshed out in terms of contributions from volume effects and phonon decay. Finally, an analytical form of the temperature dependence of the $\mathrm{A}_{1 g}$ mode is presented for the peak intensity, frequency and linewidth.

Both global and local heating on the continuous $\mathrm{CuO}$ thin films studied in the above chapter lead to a symmetrically broadened lineshape (Figure 7.3a). Chapter 9 is focused on the particular asymmetry observed in the $\mathrm{CuO}$ nanowire forest (Figure 7.3b). The disappearance of the strong asymmetry at low power implicates a thermal phenomenon: an inhomogeneous temperature distribution instead of phonon confinement or Fano resonance effects. The asymmetric lineshape is modeled using a phenomenological model that utilises the full temperature dependence derived in Chapter 8 .

The remaining part of this chapter provides the relevant introduction to the Raman spectrum of bulk $\mathrm{CuO}$ in Section 7.2, a review of the three main physical processes that can lead to asymmetric Raman lineshapes in Section 7.3 and details of sample preparation and characterisation in Section 7.4 .

\subsection{Raman spectrum of $\mathrm{CuO}$}

The Raman spectrum of $\mathrm{CuO}$ has three distinct modes at room temperature at 298, 345 and 632 $\mathrm{cm}^{-1}$. Group theory analysis on the monoclinic crystal structures shows these modes to have symmetries $A_{1 g}, \mathrm{~B}_{1 g}$ and $\mathrm{B}_{2 g}$ respectively. Numerical superscripts/subscripts help in differentiating between different modes of the same symmetry. Figure 7.4 shows that the vibrational modes are bought about by the movement of the $\mathrm{O}^{2-}$ ions alone [156, 145] while the $\mathrm{Cu}^{2+}$ remain nearly immobile. This analysis was substantiated by Irwin et al. [145] using a an isotope exchange measurement where the Raman spectra from $\mathrm{Cu}^{18} \mathrm{O}$ showed a shift in all three modes 
compared to $\mathrm{Cu}^{16} \mathrm{O}$.
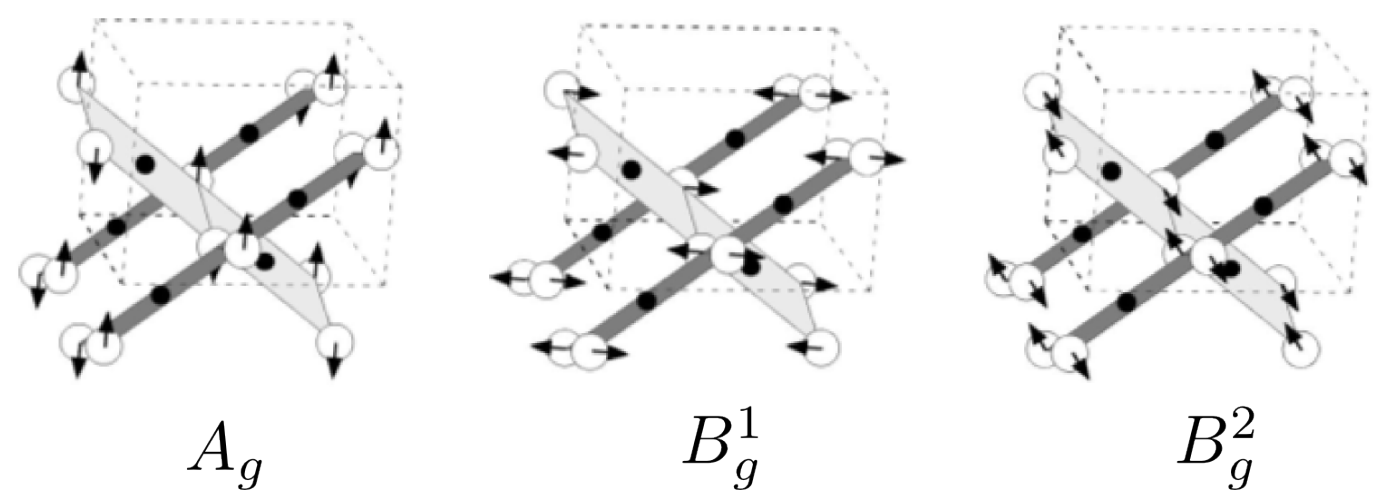

Figure 7.4: The motions of the $\mathrm{Cu}^{2+}$ (filled circles) and $\mathrm{O}^{2-}$ ions (unfilled circles) in $\mathrm{CuO}$ for the three Raman modes. Figure from [156].

According to Yamada et al. [156], the $\mathrm{CuO}$ crystal structure can be viewed as a stack of $\mathrm{CuO}$ ladders along the 110 and $\overline{1} 10$ directions. The relation between the two ladders can be represented by the intersecting and bending angles: $\theta_{1}$ and $\theta_{2} . \theta_{1}$ is the angle between the running directions of the two ladders and $\theta_{2}$ is the angle between the parallelograms in each ladder. The parallelograms here are the shape formed within the ladder by the nearest neighbour $\mathrm{O}^{2-}$ ions around every $\mathrm{Cu}^{2+}$ ion. The full three dimensional co-ordination of the oxygen ions can be viewed as a distorted octahedron (as shown by the grey hatch in Figure 7.6b). Thus, if we compare Figure 7.4 and Figure 7.6b, another view of the $A_{1 g}$ mode is that of an inter-ladder bending mode. A sample Raman spectrum of nanostructured $\mathrm{CuO}$ (Figure 7.5) shows the three Raman modes discussed above.

Aside from the $A_{1 g}$ and two $\mathrm{B}_{g}$ modes which can be assigned to a single resonance, there are two additional Raman modes which have been identified in $\mathrm{CuO}$. The first is a weak mode that appears below $213 \mathrm{~K}$ (Néel temperature) that hardens to $240 \mathrm{~cm}^{-1}$ by $60 \mathrm{~K}$. The temperature dependence characteristics of this mode have been well studied [133] and it has since been suggested as due to a strong spin-phonon interaction in $\mathrm{CuO}$ [158]. Figure 7.7a shows that this mode is not to be seen at the predicted frequency of $230 \mathrm{~cm}^{-1}$ at $-75{ }^{\circ} \mathrm{C}$. Given the weak intensity of this mode ( 0.14 relative to the intensity of $A_{1 g}$ peak at $40 \mathrm{~K}$ ) in a single crystal, it is likely that it cannot be seen in our polycrystalline $\mathrm{CuO}$ samples. The second mode is a broad feature at $1100 \mathrm{~cm}^{-1}$ (seen in the red curve of Figure 7.7b). This mode is generally associated with a multi-phonon resonance and has also been used to study confinement effects in $\mathrm{CuO}$ nanoparticles [149].

The general trend observed in both single crystal [144, 133] and nanostructured $\mathrm{CuO}$ [157, 149] is that the $A_{1 g}$ mode dominates the Raman spectrum while the $\mathrm{B}_{1 g}$ and $\mathrm{B}_{2 g}$ modes are considerably smaller and poorly resolved. The relative intensities of the $A_{1 g}$ vs the two $\mathrm{B}_{g}$ modes can be linked to polarisation dependence of the modes along different crystalline axes 


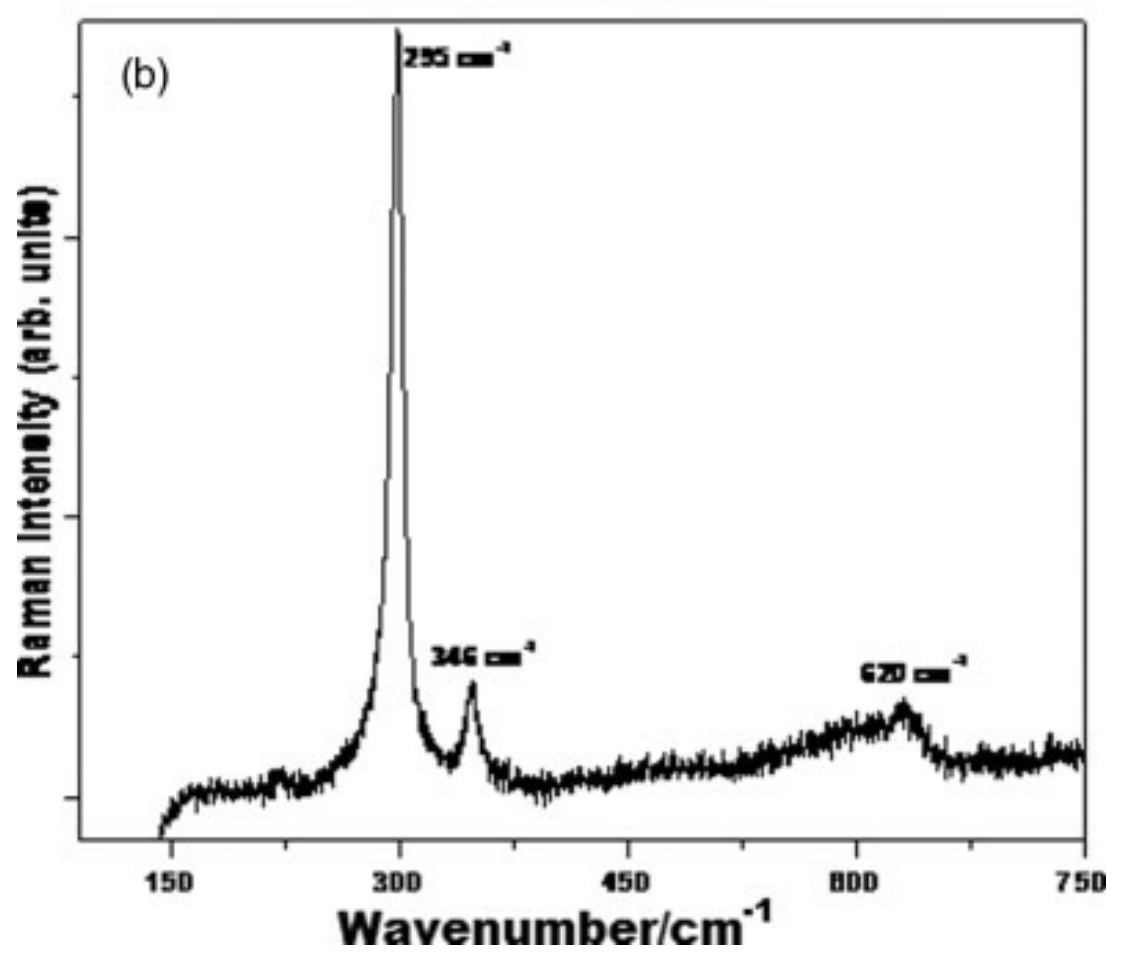

Figure 7.5: Raman spectrum of $\mathrm{CuO}$ at $632.8 \mathrm{~nm}$. Figure from [157].

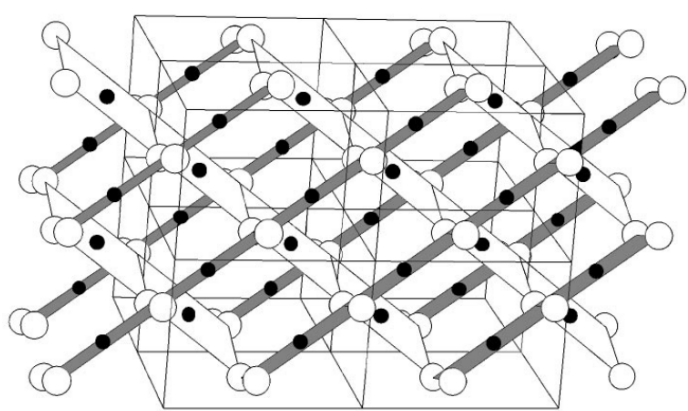

(a) $\mathrm{CuO}$ structure

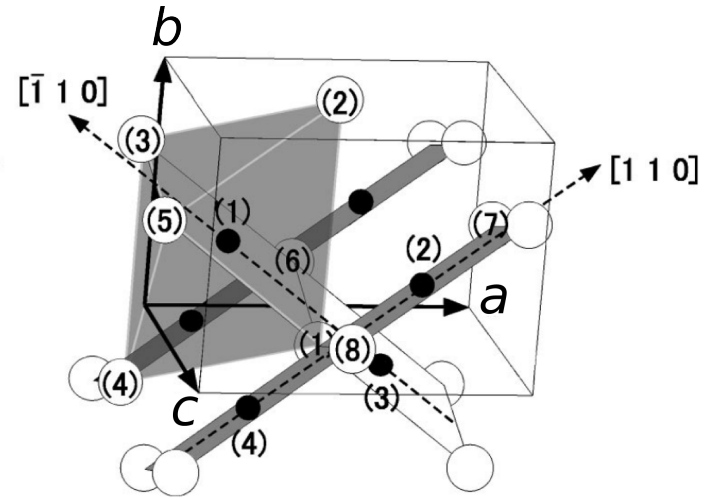

(b) $\mathrm{CuO}$ ladders

Figure 7.6: (a) $\mathrm{CuO}$ crystal. (b) $\mathrm{CuO}$ ladders within the monoclinic structure. Both figures taken from Yamada et al. [156].

[146]. There is also evidence for resonance effects from the increase in relative intensities of the two $\mathrm{B}_{g}$ modes with laser wavelength. Under the $632.8 \mathrm{~nm}$ laser, $\mathrm{B}_{1 g}$ mode intensity is just under $20 \%$ of the $A_{1 g}$ peak intensity while the $\mathrm{B}_{2 g}$ mode is barely visible (Figure 7.7a). With a $488 \mathrm{~nm}$ laser, the $\mathrm{B}_{1 g}$ peak intensity increases to $\sim 40 \%$ while the $\mathrm{B}_{2 g}$ peak increases to around $80 \%$ [157]. Under UV excitation $(325 \mathrm{~nm})$, the $\mathrm{B}_{1 g}$ and $\mathrm{B}_{2 g}$ peaks have a relative intensity of $60 \%$ and $100 \%$ respectively (Figure $7.7 \mathrm{~b}$ ).

This trend of increasing intensity with laser energy suggests a wavelength-dependent change in Raman susceptibility for the two $\mathrm{B}_{g}$ modes and could be linked to a resonance effect. An early study on single crystal $\mathrm{CuO}$ by Irwin et al. 133 . observed an increase in signal from all the Raman modes with increasing laser energy. The authors used an exploratory band structure 
study by Ching et al. [159] to suggest the onset of resonance at around $2.55 \mathrm{eV}$. The indirect and direct bandgap in $\mathrm{CuO}$ has also been independently studied using a photochemical method and found to be $1.4 \mathrm{eV}$ and $3.2 \mathrm{eV}$ respectively [132].

Given the similarity of these optical properties with that of silicon, we can utilise the wellstudied silicon Raman peak at $520 \mathrm{~cm}^{-1}$ using data from Compaan and Trodahl [17] to gain a little insight on the general trend in Raman signal with laser energy. They showed that the Raman susceptibility of the $520 \mathrm{~cm}^{-1}$ silicon peak increases steadily - over several orders of magnitude - until the direct bandgap energy of $3.32 \mathrm{eV}$ (see Chapter 2.1.1). Thus, we suggest that visible lasers used in our experiments probe $\mathrm{CuO}$ in the sub-resonance regime. Given that both the $\mathrm{B}_{g}$ modes are quite weak until the probing laser has a wavelength of $488 \mathrm{~nm}$ [157], we have chosen to focus our study on the dominant $A_{1 g}$ mode.

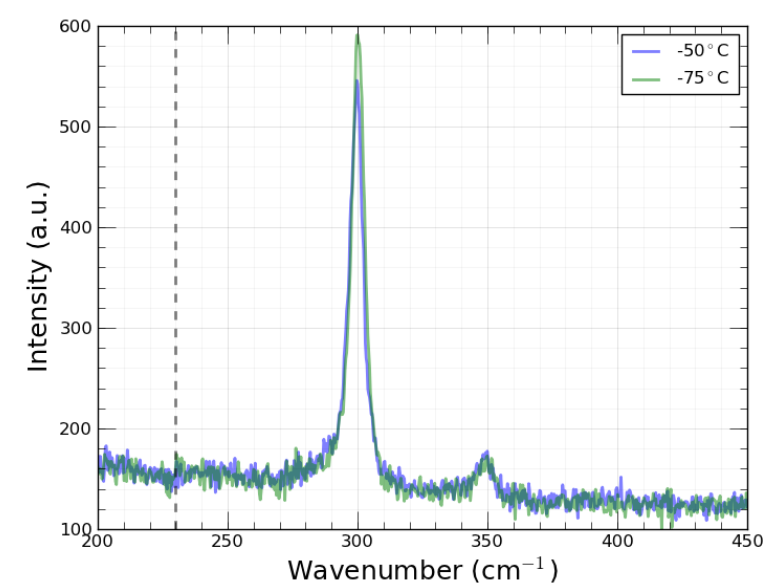

(a) Low temperature spectra

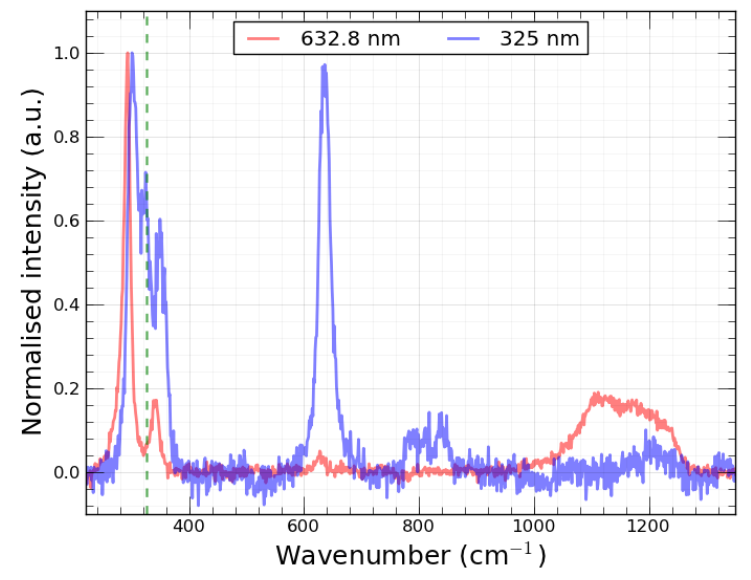

(b) $\mathrm{CuO}$ Raman: UV vs. visible

Figure 7.7: (a) Low temperature Raman spectrum of polycrystalline $\mathrm{CuO}$ thin film. The dashed line shows the expected position of the weak low temperature mode. (b) The Raman spectrum of cupric oxide in the UV $(\lambda=325 \mathrm{~nm})$ and visible $(\lambda=632.8 \mathrm{~nm})$. The dotted green line indicates the plasma line from the UV laser at $325 \mathrm{~cm}^{-1}$.

\subsection{Physical origins of lineshape asymmetry}

Phonon confinement, scattering from the carrier continuum (Fano resonance) and temperature gradients under the laser spot all cause asymmetric broadening of the Raman lineshape. The effect of the other mechanisms occurring in conjunction with each of the three mechanisms mentioned above are also discussed. The section ends with an evaluation of the likelihood that one of these three mechanisms is the dominant mechanism causing power dependent asymmetry of the $\mathrm{A}_{1 g}$ mode from a forest of cupric oxide nanowires (Figure 9.1b). 


\subsubsection{Fano resonance}

Fano resonance occurs when the background continuum of free electrons or holes interacts with the discrete excited states (Raman excited phonons) [160]. Eq. 7.1 gives the equation for the lineshape that results from this type of interaction. The parameter, $q$ ( also known as the Fano asymmetry parameter), determines if the skew is on the high $\omega$ side (positive $q$ ) or low $\omega$ side (negative $q$ ). Positive and negative $q$ are associated with holes and electrons respectively. The asymmetry parameter is also inversely proportional to carrier-phonon coupling strength where larger asymmetries (smaller $q$ ) indicate a greater coupling (Figure 7.8a). Fano lineshapes are common in bulk silicon even those without significant external doping. Figure $7.8 \mathrm{~b}$ shows the Raman spectrum from a piece of single crystal silicon used to calibrate the spectrometer prior to measurements. The observed lineshape of the $520 \mathrm{~cm}^{-1}$ mode fits well with the Fano model where the fitted value of $q$ is 21.5. This value of $q$ corresponds to a very small amount of p-doping. Figure $7.8 \mathrm{a}$ shows that this level of Fano asymmetry at $E_{\text {laser }}=1.96 \mathrm{eV}$ corresponds approximately to a hole doping concentration of $7 \times 10^{18} \mathrm{~cm}^{-3}[161]$.

$$
I(\omega)=I_{0} \frac{1+{\frac{\left(\omega-\omega_{\text {peak }}\right)}{q \Gamma}}^{2}}{1+{\frac{\left(\omega-\omega_{\text {peak }}\right)}{\Gamma}}^{2}}
$$

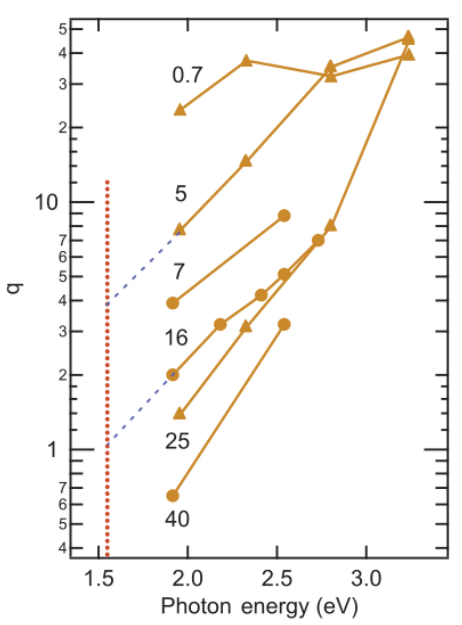

(a) Fano $q$ parameter

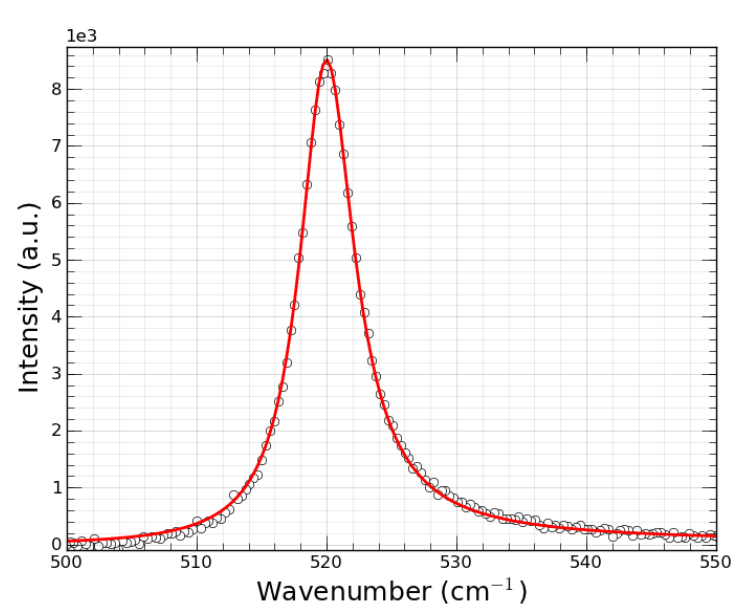

(b) Raman lineshape of bulk silicon

Figure 7.8: (a) Doping and laser energy dependence of the $q$ parameter for bulk silicon. Figure from [160]; doping concentrations (in units of $10^{19} \mathrm{~cm}^{-3}$ ) are shown in black next to curves. (b) Raman lineshape of silicon fitted with a Fano lineshape (Eq. 7.1 where $q=21.5$.

Carrier concentrations in semiconductors can be controlled by photo-stimulation. Photons with energies above the bandgap (or the activation energies of impurity levels) can activate carriers. Thus, it is possible the photo-excitation of charge carriers by the Raman laser leads to an increasing $q$ since larger carrier concentrations increase this value (Figure 7.8). Laser- 
induced Fano resonance was suggested as a mechanism for the power dependent asymmetry observed in silicon nanowires [162]. However, a subsequent study by the same group suggested that a combination of phonon confinement and inhomogeneous temperature distribution could explain the laser-stimulated asymmetry [153].

Similarly, the power dependent asymmetry of the $\mathrm{CuO}$ nanowire forest also cannot be explained by laser-induced Fano resonance. In this case, the reason is that the Fano mechanism predicts asymmetry on the wrong wavenumber side. Holes are the majority charge carriers (including carriers excited by the laser) since $\mathrm{CuO}$ is natively p-doped [130. If the hole-phonon coupling is strong (as expected for carriers with high effective mass [132]), the resultant Raman lineshape is expected to broaden on the higher wavenumber side (positive $q$ ) - opposite to the observed asymmetry. The case against Fano resonance can also be made empirically by the shape of the asymmetry. Fano lineshapes with a single peak typically has a very long, flat tail (Figure 7.8b) while the observed Raman lineshape (Figure 9.1a) does not have a discernible tail.

\subsubsection{Phonon confinement}

The visible laser photon has a wavevector, $k_{\text {laser }}$, that interacts with the Raman phonon at $k \sim$ 0 . The wavevector of the laser photon is around $10^{4} \mathrm{~cm}^{-1}$ while the Brillouin zone, $k$, of typical solids is of the order $10^{8} \mathrm{~cm}^{-1}$. The interaction region therefore is limited to $k \sim 0\left(10^{4} / 10^{8}\right)$. However, this interaction region is only relevant for bulk solids. As crystals decrease in size, the phonon momenta spread out beyond the zone center due to the Heisenberg uncertainty principle. The interaction of the laser photon is no longer confined to $k \sim 0$. Instead, the laser photon can interact with the optical mode at points along the dispersion curve with $k \geq 0$. This phenomenological model of phonon-photon interaction led Richter et al. [152] to postulate that the Raman lineshape of a confined crystal is the sum of individual lorentzians from values of $k \geq 0$ (Eq. 8.1). The contribution from a given $\mathrm{k} \neq 0$ is weighted by $\exp \left[(-k d / 2 \alpha)^{2}\right]$. This gaussian-like spectral weighting term is described by the parameter $\alpha$ whose value needs to be determined empirically. In silicon nanowires with a diameter distribution $4.5 \mathrm{~nm} \leq \bar{d} \leq 23$ nm, $\alpha=6.3$ [153]. The bottom two terms of Eq. 8.1] include the analytical form of the phonon dispersion for the specific Raman mode $(\omega(k))$ and the natural linewidth of the material $(\Gamma$ when $\mathrm{T}=0 \mathrm{~K})$.

$$
I(\omega)=\underbrace{\mathrm{Z} 1}_{0} \frac{2 \pi k \exp -\frac{k d}{2 \alpha}^{2}}{[\omega-\omega(k)]^{2}+\left(\Gamma_{0} / 2\right)^{2}} d k
$$

We can qualitatively simulate the expected asymmetry of the $\mathrm{A}_{1 g}$ lineshape of $\mathrm{CuO}$ due to phonon confinement using Eq. 8.1. For this simulation, $\omega(k)$ is obtained from calculated phonon 
dispersion curves of Reichardt et al. [163] (shown in Figure 7.9a). The curve is described with a simple cosine: $\omega(k)=a+b \cos (\pi k)$ where $a=289.5 \mathrm{~cm}^{-1}$ and $b=33.4 \mathrm{~cm}^{-1}$. For the remaining parameters, $\Gamma_{0}$ is set to $2.928 \mathrm{~cm}^{-1}$ (from the the temperature dependence measurements in Chapter 8.2) and $\alpha$ is arbitrarily chosen to be 1.5. It is worth noting that larger values of $\alpha$ lead to a larger asymmetry since increasing values of $\alpha$ result in a greater sampling of the phonon dispersion curve (Figure 7.9b). Figure 7.9c shows the Raman lineshapes obtained for three different diameters using the above parameters.

The asymmetry due to phonon confinement qualitatively matches the asymmetry seen in Figure 9.1b, However, there is one major discrepancy between the experimentally observed and modelled $\mathrm{A}_{1 g}$ lineshapes: the peak frequency of the $\mathrm{A}_{1 g}$ mode. This mismatch arises from the phonon dispersion data. The calculated dispersion curves of Reichardt et al. shown in Figure $7.9 \mathrm{a}$ give the $\mathrm{A}_{1 g}$ phonon frequency at the zone center as $320 \mathrm{~cm}^{-1}$; a value that is substantially higher than the limiting frequency of $303.7 \mathrm{~cm}^{-1}$ calculated from the temperature dependence data. The discrepancy is the result of dispersion curves calculated along different directions of the Brillouin zone. The dispersion curve in Figure 7.9a is calculated along the $a$ axis of $\mathrm{CuO}$ while the $\mathrm{CuO}$ nanowires are polycrystalline. Another minor consequence of the phonon dispersion data is the non-monotonous decrease in $\omega(k)$. If the spectral weighting term samples $k>0.5$, the simulated Raman lineshape will begin to show spikes (since the phonon frequency starts to increase for $k>0.5)$.

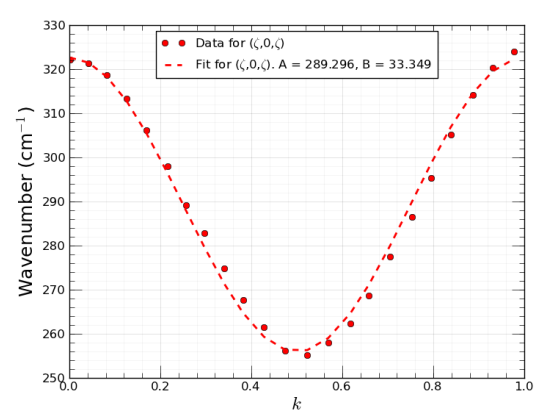

(a) $\mathrm{CuO}$ dispersion relation

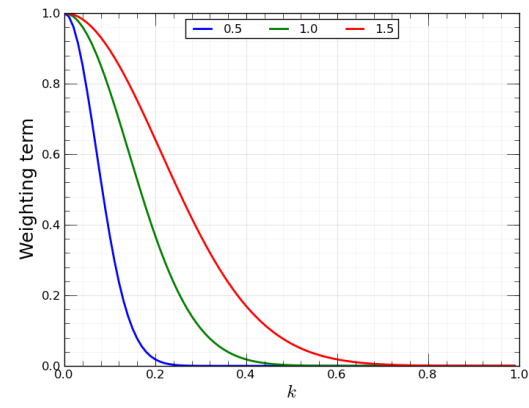

(b) Spectral weighting

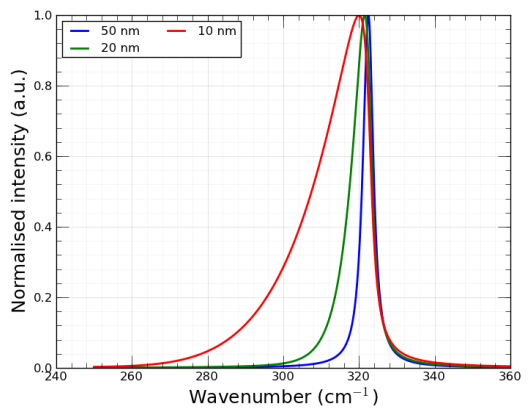

(c) Resultant spectra

Figure 7.9: (a) Phonon dispersion along the $(\zeta, 0, \zeta)$ direction of $\mathrm{CuO}$ calculated in [163]. (b) Spectral weighting with different values of $\alpha$. (c) Simulated $\mathrm{A}_{1 g}$ lineshape using $\alpha=1.5$ for three different diameters.

Despite the poor fit to the data, simulating the effects of phonon confinement on the $A_{1 g}$ mode shows two things: (i) the shape of the asymmetry matches that seen in the $\mathrm{CuO}$ nanowire forest and (ii) choosing appropriate $\omega(k)$ and $\alpha$ are critical for accurate modelling of the observed Raman lineshape. The $\alpha$ parameter is particularly critical since it dictates the degree of asymmetry. An arbitrary value of $\alpha=1.5$ gives the onset of phonon confinement for confined dimensions smaller than $\sim 20 \mathrm{~nm}$. Typically, the Bohr radius $\left(a_{B}\right)$ of a material is a reasonably accurate indicator of the critical dimension below which confinement effects become prominent. 
It can be calculated using the hydrogen Bohr radius formula:

$$
a_{B}=\frac{4 \pi \varepsilon_{0} \hbar^{2}}{m_{0} q^{2}}
$$

where $\varepsilon_{0}, \hbar, m_{0}$ and $q$ are the permittivity of free space, reduced Planck's constant, electron rest mass and elementary charge. The hydrogen Bohr radius calculated using Eq. 7.3 equals $0.53 \AA$. The Bohr radius for hydrogenic impurities in a given material can be calculated by multiplying the hydrogen Bohr radius (the above equation) by the dielectric constant $\kappa$ and dividing by the effective mass $\left(m_{\text {eff }}\right)$ of the material. While phonon confinement is a simple model that describes odd Raman lineshapes, it is too often used without appropriate consideration of other mechanisms [148, 149, 150]; thermal phenomena in particular. A recent study on silicon nanocrystals showed that phonon confinement effects on the Raman spectra can only be observed when dimensions are below $10 \mathrm{~nm}$ (the silicon Bohr radius is $5 \mathrm{~nm}$ ) [154]. Above $10 \mathrm{~nm}$, thermal phenomena are the major cause of lineshape broadening. The dominance of heating effects is due to the suppression of thermal transport by boundary scattering mechanisms that dominate in nanomaterials; even when the dimensions are above the confinement regime [155, 164]. For example, the thermal conductivity of a silicon nanowire with a diameter of $22 \mathrm{~nm}$ is around 5 times lower than that of wire with diameter $115 \mathrm{~nm}$ [164. The reduction in heat transport both through the sample and a poor thermally conducting medium like air [165] causes a significant rise in temperatures using moderate laser powers.

Thus, modelling Raman spectra from nanomaterials with confined dimensions close to or below the Bohr radius must also account for laser heating effects. The temperature induced by the laser beam can be spatially homogeneous [166] or inhomogeneous [153]. In the former, a symmetric broadening of the peak is convoluted with the asymmetric broadening due to the phonon confinement. Figure 7.10a shows the Raman spectrum of the TO mode in germanium nanowires with an average diameter of $6 \mathrm{~nm}$. In this case, the confined dimensions are well below the Bohr radius of germanium $(\sim 17 \mathrm{~nm})$ and phonon confinement is the dominant mechanism. However, the authors' still need to modify the phonon confinement model given in Eq 8.1 to account for a uniform heating of the nanowires by the laser beam.

Inhomogeneous heating by the laser provides an additional asymmetry to a lineshape already asymmetrically broadened due to phonon confinement. Adu et al. showed that long, individual silicon nanowires on a poor heat sink show considerable degree of inhomogeneous heating [153]. The inhomogeneous temperature distribution is modelled using a simple exponential model: 


$$
T(z)=\Delta T \exp -\frac{z}{b}^{2}+T_{a}
$$

The parameter $b$, is estimated using the power dependence of the asymmetry component. Since the asymmetry due to phonon confinement is power-independent, any changes with power are attributed to a spatially inhomogeneous temperature. Temperature gradients in the sample are directly related to the gaussian intensity distribution. Raman lineshapes are calculated using a modified form of Eq. 8.1. This equation (Eq. 7.5) states that the lineshape asymmetry is both due to temperature gradients and phonon confinement. The Raman lineshape is calculated by integrating over the phase space of temperature and $k$.

$$
I(\omega)={ }_{-c}^{Z^{c}} I_{0} \exp -\frac{z}{a}{ }^{2} d z \sum_{0}^{Z_{1}} \frac{2 \pi k \exp -\frac{k d}{2 \alpha}^{2^{\mathbf{i}}}}{[\omega-\omega(k, T(z))]^{2}+\left(\Gamma(T(z) / 2)^{2}\right.} d k
$$

The fits to Eq. 7.5 are shown for two nanowire samples: one with an average diameter close to $(\bar{d} \sim 6 \mathrm{~nm}$; Figure 7.10c) and above $(\bar{d} \sim 23 \mathrm{~nm}$; Figure $7.10 \mathrm{~b})$ the silicon Bohr radius. A close inspection of the observed Raman lineshape for the larger diameter silicon nanowires reveals a symmetric rather than asymmetric broadening at high laser power and Eq. 7.5 over-estimates the asymmetry. Adu et al. suggest that the inhomogeneous temperature distribution alone is responsible in this case since the phonon confinement model will not give any asymmetry. At low laser powers, the data for both 6 and $23 \mathrm{~nm}$ nanowires appear symmetric while the fitted lineshape is asymmetric. These poor fits suggest that Eq. 7.5 is applicable only for spectra from confined nanowires taken at high power. Simpler models like those of pure homogeneous and inhomogeneous heating could account for the remaining spectra.

Phonon confinement effects can be considered negligible in the $\mathrm{CuO}$ nanowire forest due to the power dependence spectra shown in Figure 9.1. The recovery of the $\mathrm{A}_{1 g}$ mode at low power to a symmetric lineshape similar to that of the thin film is a clear of a thermal phenomenon as the low power lineshape remain asymmetric if phonon confinement were the underlying mechanism. Furthermore, the Bohr radius for $\mathrm{CuO}$ is calculated to be $\sim 0.5 \mathrm{~nm}$ using an effective mass of $1.87 m_{0}$ (calculated using hole and electron masses from[130]) and a dielectric cosntant, $\kappa$, of 18.1 [167]. While this value is only an estimate, the key point is that it is quite small. $\mathrm{CuO}$ nanowires prepared by thermal oxidation have diameters well above this value [137]. Thus, the power-dependent asymmetry and small Bohr radius of $\mathrm{CuO}$ show that phonon confinement is unlikely to explain lineshape asymmetry. This leaves temperature inhomogeneity as the most 


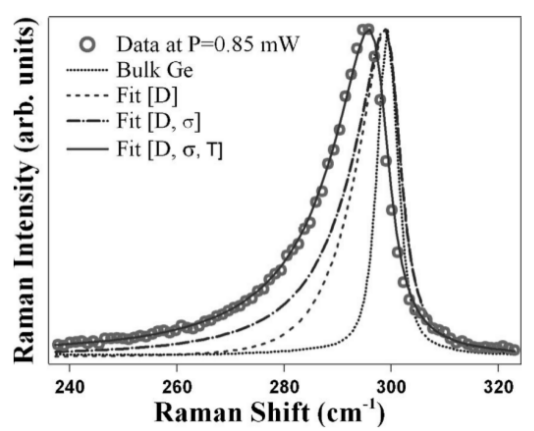

(a) Homogeneous heating

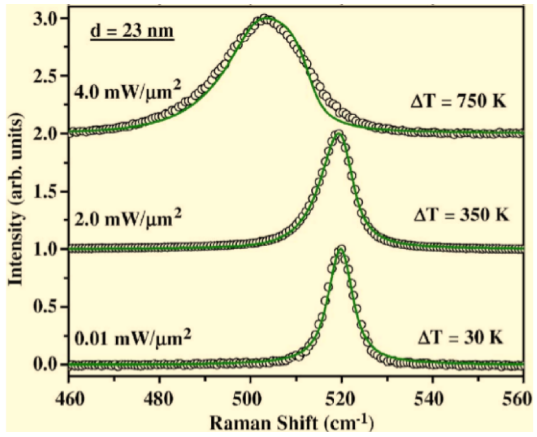

(b) Inhomogeneous heating. $\bar{d}=23$ (c) Inhomogeneous heating. $\bar{d}=6$ nm.

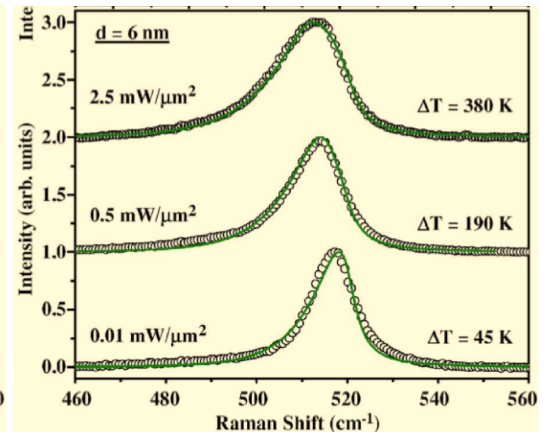

nm.

Figure 7.10: (a) Phonon confinement model + homogeneous heating for Ge nanowires with $\bar{d} \sim$ $6 \mathrm{~nm}$. Figure from [166] (b) Phonon confinement model + inhomogeneous heating for a $6 \mathrm{~nm}$ silicon nanowire. Figure from [153]. (c) Phonon confinement model + inhomogeneous heating for a $6 \mathrm{~nm}$ silicon nanowire. Figure from [153].

plausible mechanism of the power-dependent asymmetry. In Chapter 9, we examine the power dependence of the lineshape asymmetry in detail and, demonstrate an extension of the homogeneous heating results from the previous chapter to model the Raman signal from a sample with a temperature distribution. As will be seen in the following chapters, our approach provides a physical basis for lineshape asymmetry due to temperature gradients, a facet that was not explored in the preliminary model of Adu et al.

\subsection{Sample preparation and characterisation}

To obtain a monophase $\mathrm{CuO}$ film, a film of copper was annealed at moderately high temperatures. Previous work showed that oxidation temperatures below $400{ }^{\circ} \mathrm{C}$ produced mixed phase films [134]. The procedure for fabricating nanowires is less straightforward since the quality of growth is dependent upon temperature, annealing time, the chosen copper substrate and a more ephemereal parameter of local air flow fields around the substrate during annealing [168]. A few general principles can be gleaned from existing literatue such as the inverse proportionality of diameter with growth temperature and the linear dependence of nanowire lengths with growth time [169, 137, 170, 171]. With these general guidelines, the methodology has to be fine-tuned for the particular fabrication conditions. For this I am grateful for the considerable preliminary work by Honours student James Eldridge, who established the basic procedure. When successful, nanowire forest growth is seen as a velvety flaky surface over the copper substrate (Figure 7.11). Using the experimental conditions described below the success rate for fabricating samples with some nanowire coverage was quite high. In most cases, the entire copper button could be covered as shown in Figure 7.11 .

$\mathrm{CuO}$ samples for Raman analysis were prepared by one of two methods: thermal oxidation 


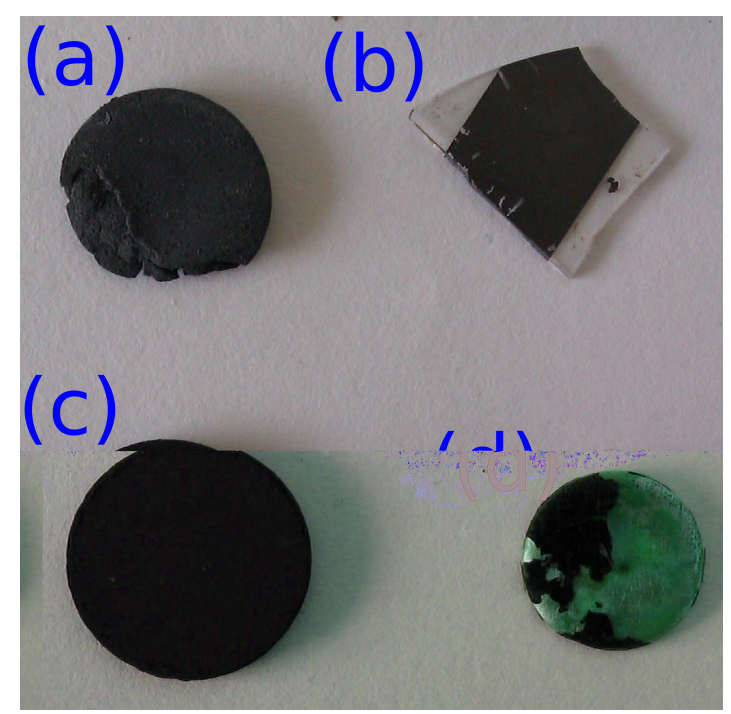

Figure 7.11: (a) Sintered pellet. (b) Thin film. (c) Unsintered pellet. (d) Nanowire forest.

and pressing. The nanowire forest and thin film were fabricated by annealing copper subtrates in air. For the former, a copper button (cleaned in $\mathrm{HCl}$ and de-ionised $\mathrm{H}_{2} \mathrm{O}$ ) was annealed at $475{ }^{\circ} \mathrm{C}$ with a gentle flow of air running $(110 \mathrm{~mL} / \mathrm{min})$ through a water bubbler. Water vapour has been shown to facilitate the nucleation of nanowires [172]. The thin film was made in two steps. First, a layer of copper of thickness $\sim 1 \mu \mathrm{m}$ was evaporated using an Edwards 305A thermal evaporator onto double side polished sapphire. This film was then annealed in a tube furnace without air flow at $500{ }^{\circ} \mathrm{C}$ for two hours. The pellet was fabricated by pressing $\sim 0.7$ $\mathrm{g}$ of cupric oxide powder from Aldrich with a force of 8.5 tonnes across a $13 \mathrm{~mm}$ die (equal to a pressure of $6.24 \mathrm{kbar})$. The pellet was sintered in a furnace for 18 hours at $950{ }^{\circ} \mathrm{C}$.

\subsubsection{XRD}

Powder XRD patterns from the pellets and thin film were obtained using an X'Pert Pro Diffractometer in the Bragg-Brentano geometry. For the $\mathrm{CuO}$ thin film, four runs from $20^{\circ}<2 \theta<$ $100^{\circ}$ were averaged to obtain a good signal to noise ratio. The position of the sapphire (006) reflection from the sample substrate was matched to a control diffractogram to ensure the sample was properly aligned. The diffraction pattern from the thin film is shown in Figure 7.12 along with a reference from the PDF database [129]. All peaks except the one marked with an asterix can be attributed to $\mathrm{CuO}$. The gap between $40-44^{\circ}$ is due to the removal of the sapphire (006) signal which would otherwise overwhelm the $\mathrm{CuO}$ diffraction pattern. For all subsequent analyses, only the peaks from $32-65^{\circ}$ were used since most of these are just comprised of signal from one plane. The higher $2 \theta$ peaks are less desirable for analysis, as they are too weak and too many signals are clustered together within the peaks.

The diffractograms from the two pellets displayed a constant shift of $\sim 0.5^{\circ}$ which corresponded to a displacement factor due to inappropriate sample holders. The position of the 


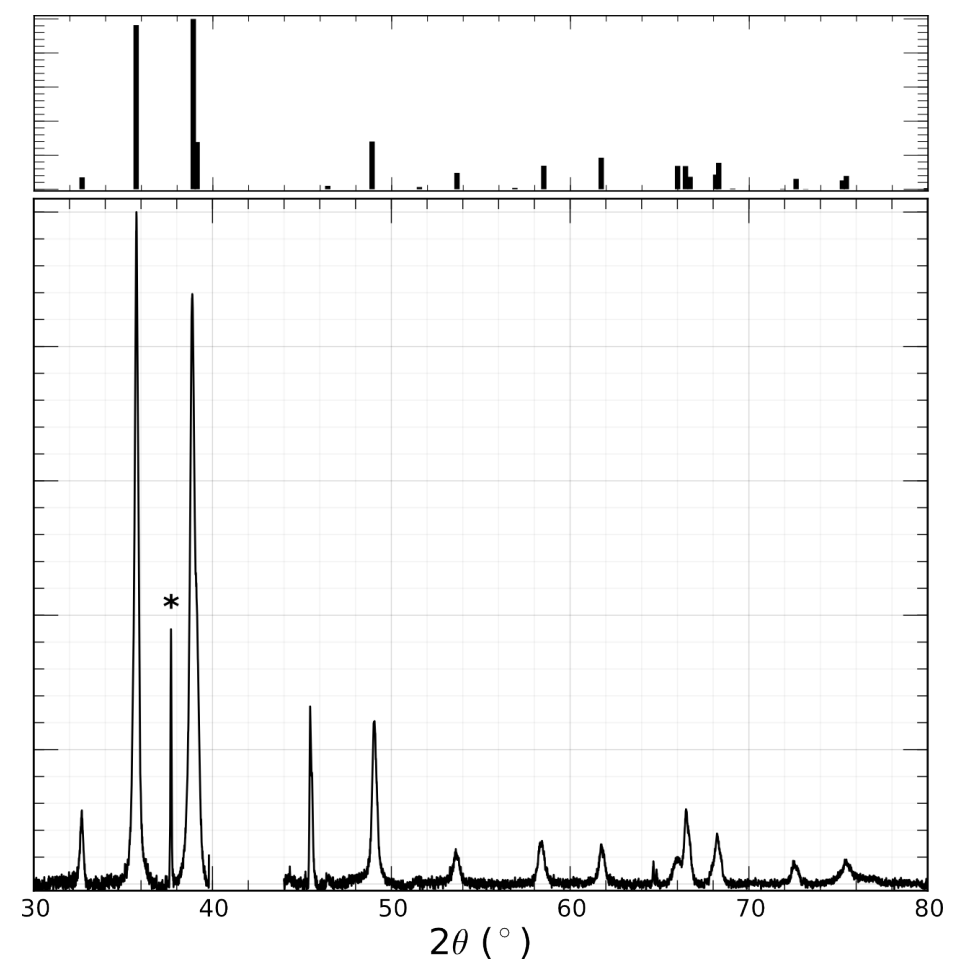

Figure 7.12: $\mathrm{CuO}$ XRD pattern. The top plot shows the reference pattern for $\mathrm{CuO}$ [129].

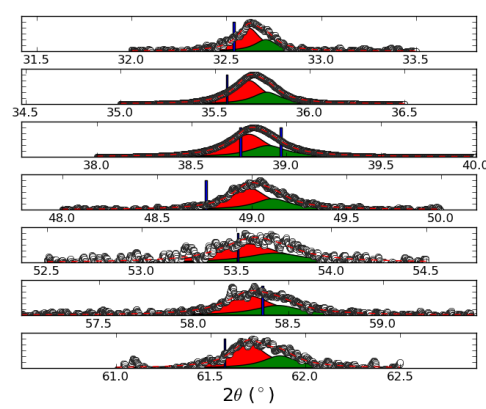

(a) Thin film

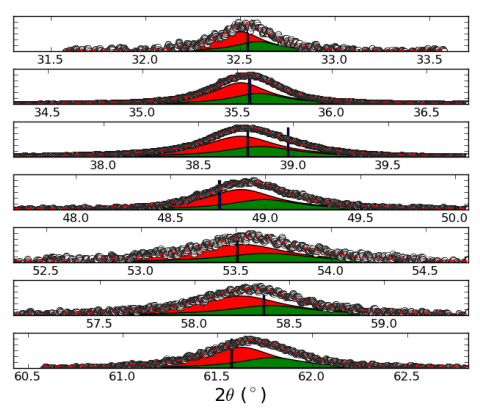

(b) Unsintered pellet

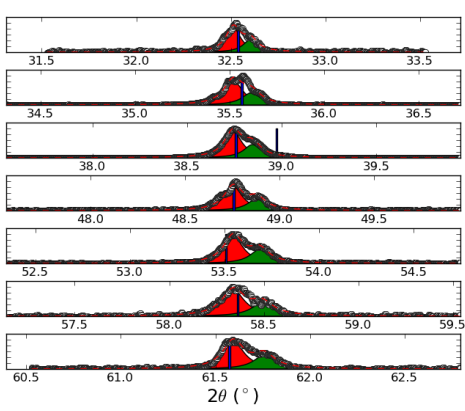

(c) Sintered pellet

Figure 7.13: Diffractograms and two lorentzian fits of seven common XRD peaks.

two samples from the expected illumination plane were measured to be around $1.5 \mathrm{~mm}$. A more rigorous correction for the non-ideal displacement was established using experimental data plotting position shift of $\mathrm{Al}_{2} \mathrm{O}_{3}$ vs. displacement [173]. The data shows a linear relationship between the position shift and the sample displacement. These experimental values were used to estimate the displacement of the pellets from the measured position shift of the $\mathrm{CuO}$ pellets from the PDF reference pattern. The average displacement was around $1.2 \mathrm{~mm}$ for both samples which agrees very well with the measured values. Hence, the average shift was used to correct for the displacement factor in each peak. The corrected diffractogram data are shown in Figure $7.13 \mathrm{~b}$ and $7.13 \mathrm{c}$.

Figure 7.13 shows the fitted peaks for seven main reflections of the unsintered and sintered pellets and the thin film. The fitting was performed manually where each reflection was isolated 
and background corrected before being fitted with a peak lineshape that is a linear combination of two Lorentzians. Unlike the silicon peaks, the $\mathrm{CuO}$ peaks did not exhibit any observable asymmetry. For the broader peaks, it was necessary to constrain the fitting such that the $k \alpha_{2}$ is included in the fitting. This was done by constraining the position and intensity of the second Lorentzian. The position was fixed using the expected shift between $k \alpha_{1}$ and $k \alpha_{2}$ (Chapter 2.5) and intensity was fixed such that it was always half that of the $k \alpha_{1}$ peak. This fitting procedure allows the low $2 \theta$ reflections with insufficient separation between the $\mathrm{k} \alpha_{1}$ and $\mathrm{k} \alpha_{2}$ peaks to be included in the analysis. This is especially important for $\mathrm{CuO}$ as the dominant reflections are in the low $2 \theta$ region.

Figures $7.13 \mathrm{a}$ and $7.13 \mathrm{~b}$ show that there are significant shifts in the peak positions compared to the reference spectra for $\mathrm{CuO}$. Since these shifts are not the result of calibration errors - the sapphire control peak at $\sim 40^{\circ}$ was matched to the sapphire peak of the sample - they indicate some compressive lattice strain within the $\mathrm{CuO}$ film. The presence of compressive strain in the $\mathrm{CuO}$ film is not surprising as the total film volume increases by more than a factor of two during the thermal oxidation process. As such, any increase in crystallite size that occurs parallel to the substrate plane will hence lead to compressive stress in the matrix. The apparent strain derived from the position shift appears to be a strongly dependent on the $(h k l)$ plane, indicating anisotropic strain broadening of specific $(h k l)$ planes. We also see an anisotropic dependence of the linewidth on 2 $\theta$. This is similar to observations by Bianchi et al.[174] who also attribute this effect to anisotropic strain in $\mathrm{CuO}$ powder.

$$
\begin{gathered}
\beta=\frac{k \lambda}{D_{v} \cos (\theta)} \\
\beta \cos (\theta)=\frac{k \lambda}{D_{v}}+4 \varepsilon \sin (\theta)
\end{gathered}
$$

Further analysis of the peak linewidth $(\Gamma)$ was carried out after correcting for instrumental broadening using the relation for Lorentzian lineshapes: $\Gamma=\Gamma_{\text {obs }}-\Gamma_{\text {instrument }}$. These corrected values were then further analysed using the crystallite size analysis relations of Scherrer (Eq. 7.6) and Williamson-Hall (Eq. 7.7). The parameters $\mathrm{k}, \lambda, D_{v}$, and $\varepsilon$ are the crystallite shape factor (0.9 for spherical particles), the $\mathrm{Cu} \mathrm{K} \alpha_{1}$ wavelength $(0.15405980 \mathrm{~nm})$, the volumeaveraged crystallite size and the strain respectively. $\beta$ is the Lorentzian linewidth converted into an integral breadth using the formula: $\beta=(\pi / 2)^{*} \Gamma$.

The Scherrer equation, without strain correction, yields an average particle size across all seven reflections of $36 \pm 5 \mathrm{~nm}$, with a large variation between peaks from $60 \mathrm{~nm}$ to $20 \mathrm{~nm}$. 


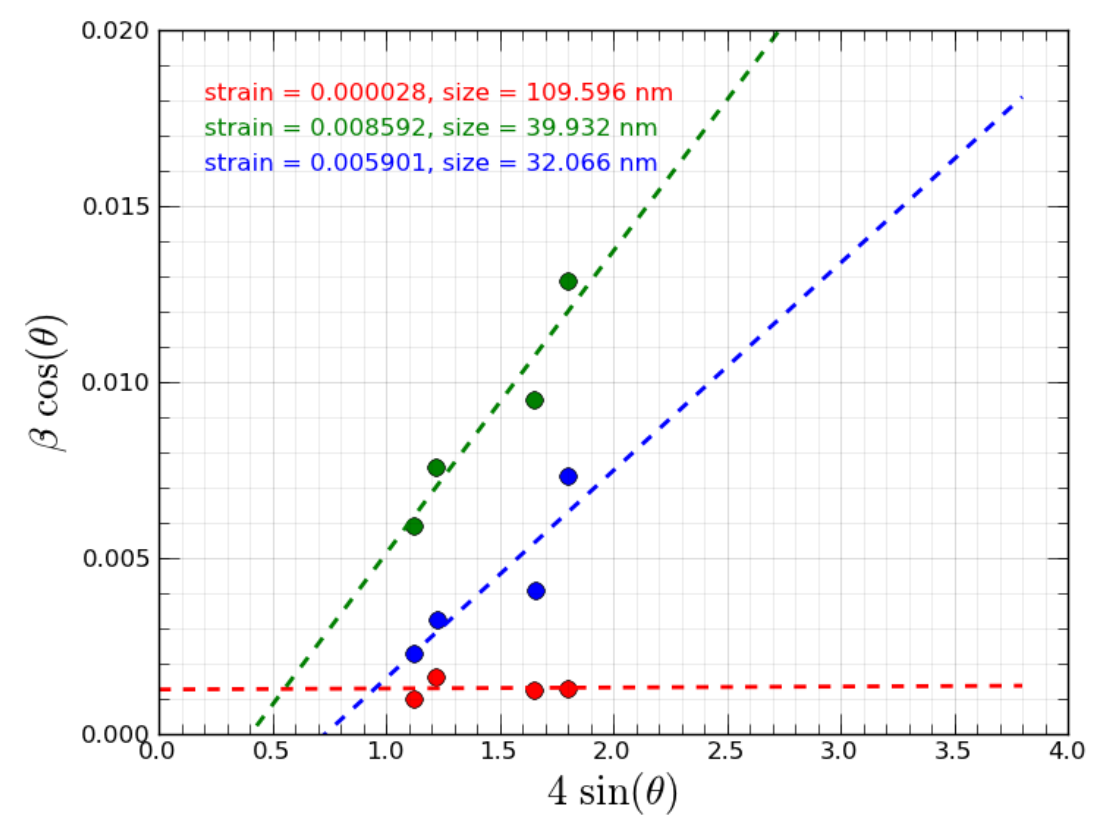

Figure 7.14: Williamson-Hall analysis for all three samples. (red $=$ sintered pellet, green $=$ unsintered pellet, blue $=$ thin film).

To account for strain broadening, Williamson-Hall analysis was performed. Using the FWHM data from all seven reflections in the Williamson-Hall plot gives a strain of $2.25 \times 10^{-3}$ and a crystallite size of $200 \mathrm{~nm}$. However, using all seven reflections is not robust since the $\mathrm{R}^{2}$, or goodness-of-fit, is around 0.25. Instead, we chose to apply the Williamson-Hall analysis to a subset of the reflections. This approach is justified because of the large noise fluctuations and very low intensities for some of the weaker reflections eg. at $58.3^{\circ}$ and $61.7^{\circ}$. The dominant reflection at $38.8^{\circ}$ is also left out of the analysis since this composite peak is most likely a composite of two reflections. The doublet cannot be resolved due to the presence of the $\mathrm{k} \alpha_{2}$ peak. Figure 7.14 shows the Williamson-Hall analysis for all four reflections, indicating a similar average particle size of $32 \mathrm{~nm}$ to that obtained via Scherrer analysis and a larger average crystal strain than that calculated from the peak position shifts.

The Williamson-Hall analysis is generally performed when strain and size broaden the peaks while the Scherrer analysis applies when strain is small. Scherrer analysis of a material with a reasonable strain contribution to the peak broadening will give a smaller grain size compared to a Williamson-Hall analysis. Given the agreement in the grain size using with these two methods, the contribution of strain can be taken as negligible in all three samples.

\subsubsection{Conduction in $\mathrm{CuO}$ thin films}

Figure 7.15 shows current vs. temperature (plotted in the Arrhenius style) data measured at different voltages for a $\mathrm{CuO}$ thin film. The strong linearity in the data shows that thermal activation is the dominant conduction mechanism. The invariant slope for voltages between 


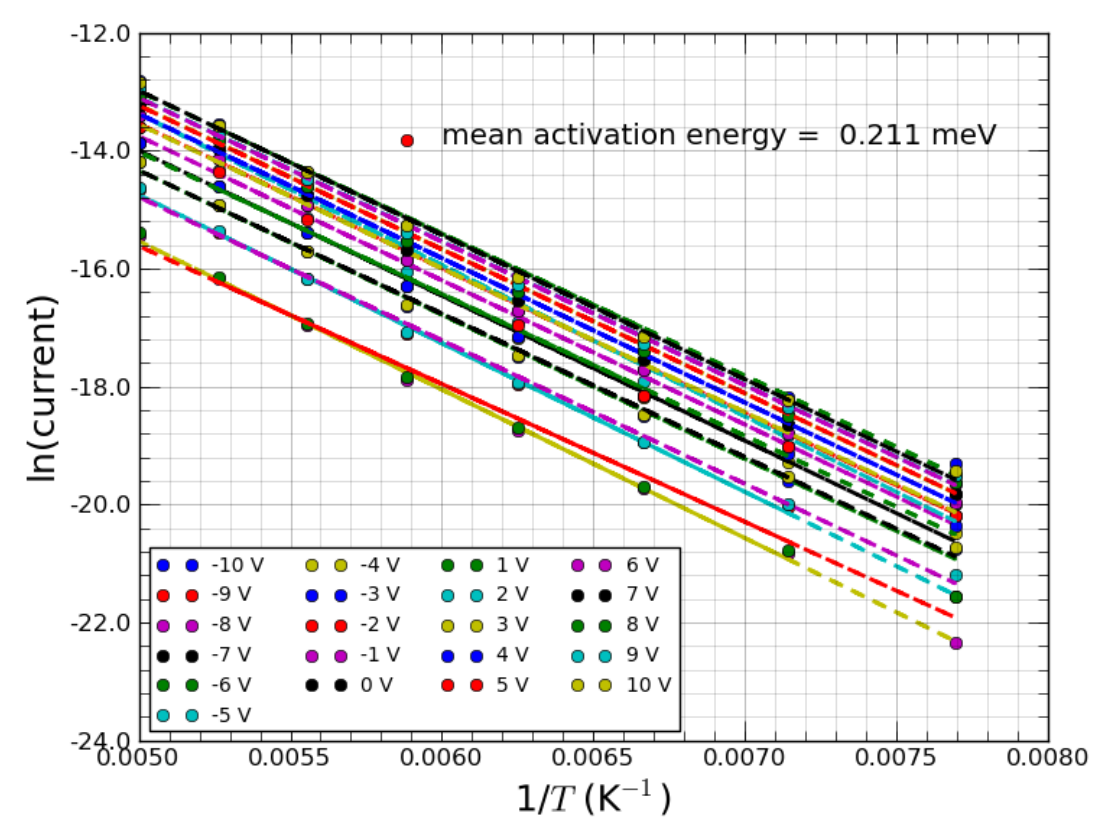

Figure 7.15: Arrhenius plots of current vs. temperature (between 200 and $130 \mathrm{~K}$ ) at different voltages for a $\mathrm{CuO}$ thin film.

$\pm 10 \mathrm{~V}$, indicates that our thin films of nanocrystalline $\mathrm{CuO}$ have a voltage-independent thermal activation energy of $\sim 0.2 \mathrm{eV}$. Furthermore, this lack of correlation between the voltage and the activation energy attests to activation processes within the film, not due to Schottky barriers at the metal- $\mathrm{CuO}$ contacts.

Previous studies [175, 176, 177] have proposed the presence of conduction barriers that are associated with depletion regions at grain boundaries in microcrystalline $\mathrm{CuO}$. However, the sub-100 nm diameter of our nanocrystallites suggest the entire nanocrystal would be depleted in our films and would not participate in conduction processes. Given the p-type nature of our films we attribute the activation behaviour to trap states that lie $\sim 00.2 \mathrm{eV}$ from the valence band edge. These trap states can be associated with $\mathrm{Cu}^{2+}$ vacancies [130. Figueirido et al. [134] have also reported similar activation energies of $0.1-0.16 \mathrm{eV}$ for $\mathrm{CuO}$ films with crystallite diameters of $<1$ mum.

\subsubsection{Morphology}

\section{Thin film}

The thin film has a very interesting morphology due to the confined expansion of the evaporated copper film. The expansion in the unit cell from copper to cupric oxide is significant $(2.7$ change in cell volume). However, the oxidation process that we have followed does not seem to expand the film uniformly and instead creates a geographic landscape of hills and valleys. The size of these hills are significant enough to be seen in the microscope (Figure 7.16a). Coarse 


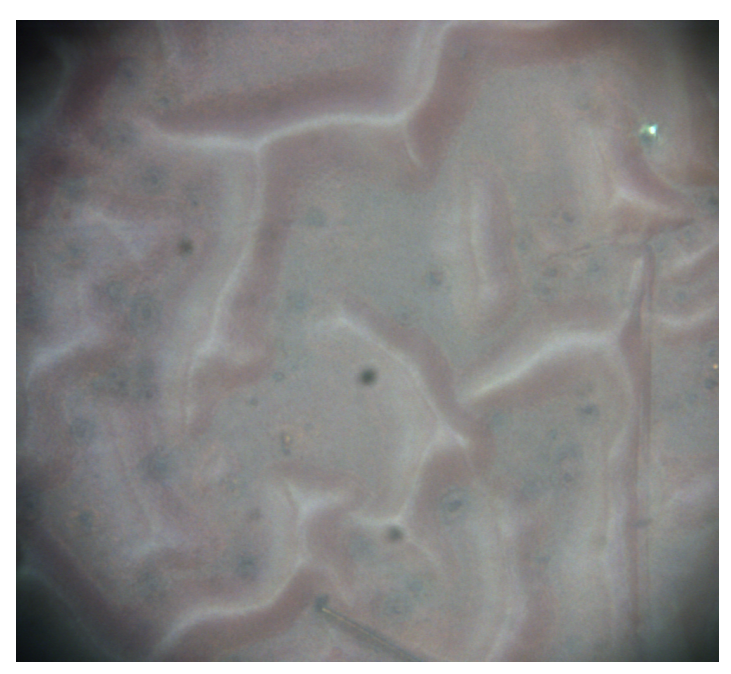

(a) Optical micrograph

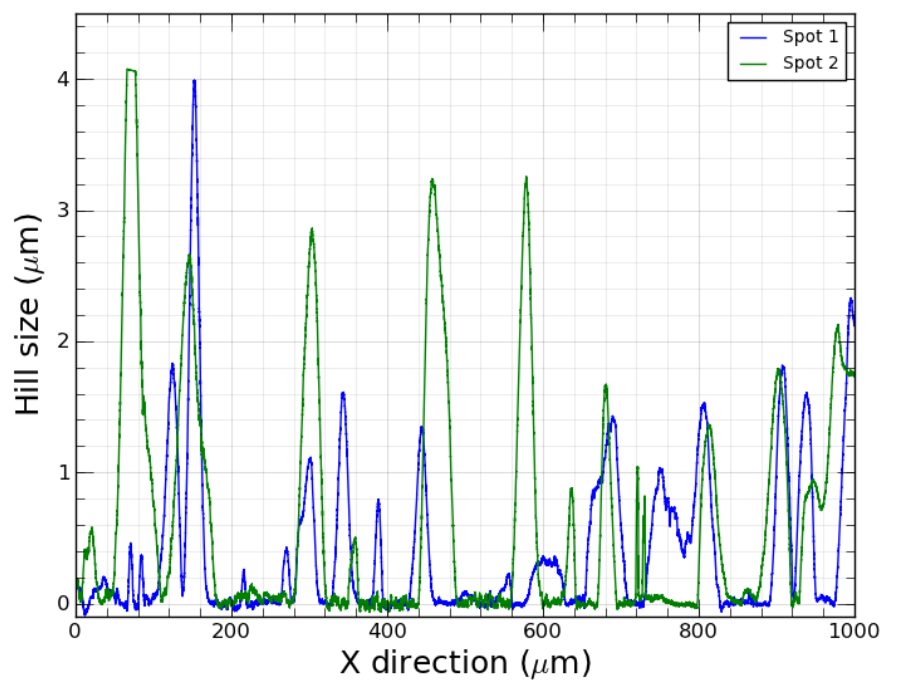

(b) Coarse contact microscopy

Figure 7.16: The varying geography of the thin film landscape. The hills are large enough to be seen in a microscope.

contact microscopy using the Dektak shows that the hills can be up to $4 \mu \mathrm{m}$ in height from the valley floor (Figure 7.16b). The low resolution SEM image in Figure 7.17a also shows the same hill and valley geography seen in the optical image and the Dektak profile. The implications of this variable sample morphology have considerable repercussions on the Raman data.

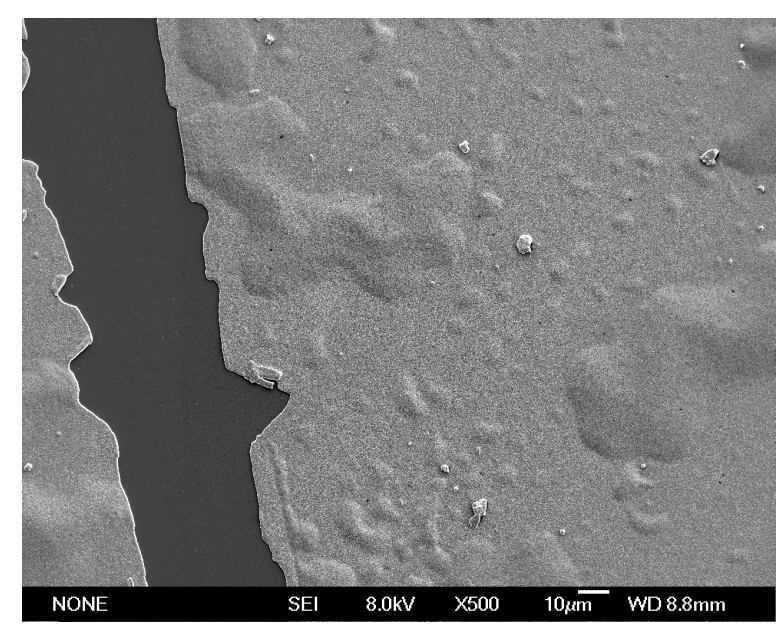

(a) Low magnification

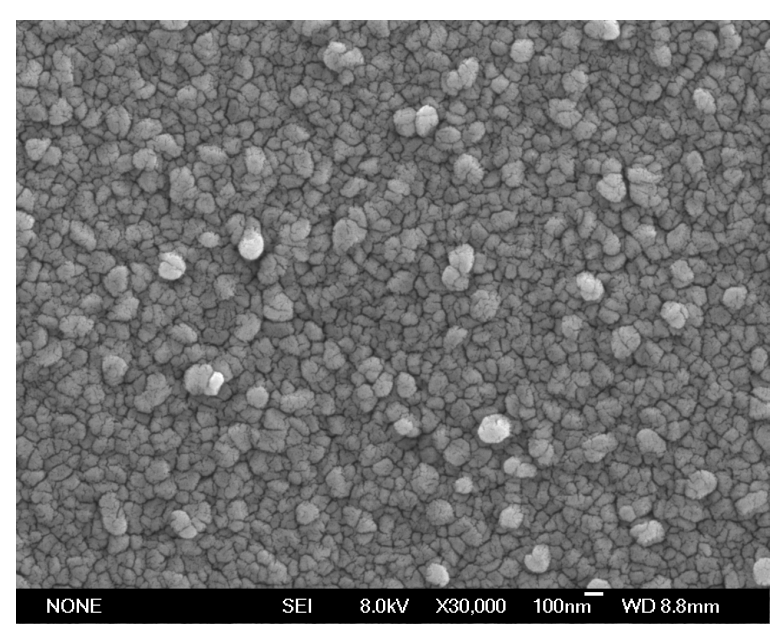

(b) High magnification

Figure 7.17: SEM images of CuO thin film morphology at two different length scales

High resolution SEM images of the $\mathrm{CuO}$ thin film (Figure 7.17b) show a nanostructured surface comprised of many 'nearly' spherical nanoparticles. It is instructive to compare the particle sizes obtained from image analysis with the volume-averaged crystallite sizes obtained from our XRD analysis. Image analysis - once optimised to remove artefacts and false positives - can be a powerful tool for estimating grain size distributions since it is directly sampling the 
sample morphology. Image analysis can also give information relating to the shape factor of the grains which makes it considerably more useful than XRD analysis which assumes spherical grain sizes and can only handle monomodal size distributions. A number of image analysis techniques can be used to extract size distributions from high resolution SEM images. We have used the 'Analyze Particles' feature in ImageJ to analyse our images.

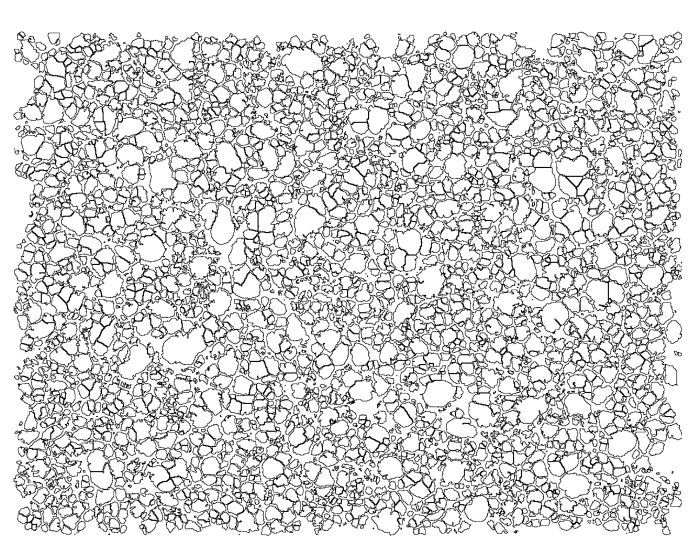

(a) Image J Analysis

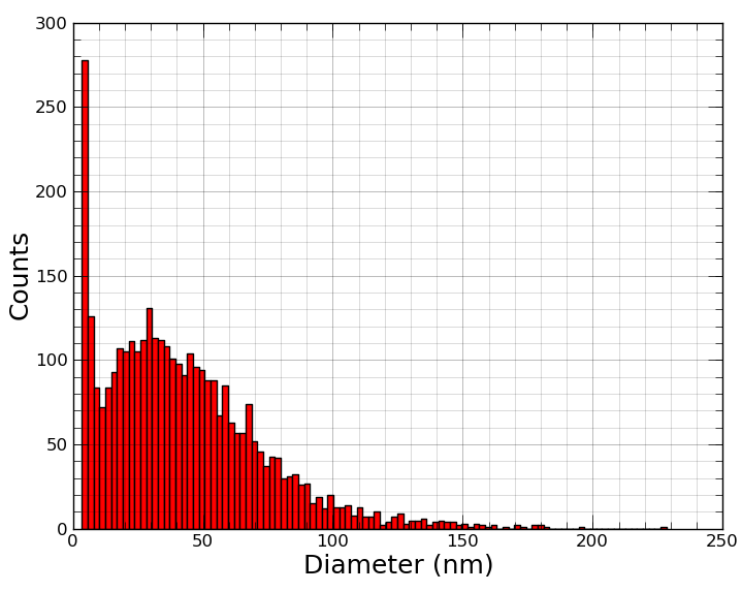

(b) Grain size histogram

Figure 7.18: (a) ImageJ analysis of the SEM image shown in Figure 7.17b and the histrogram of particle sizes from that analysis (b).

The SEM image in Figure 7.17b (obtained at 30,000×) was analysed using the freely available and widely used software, ImageJ [178]. First, the image was thresholded such that greyscale values above 100 were made 0 . This had the effect of changing the image such that each observable grain was black with a white outline. A watershedding algorithm was then implemented to segment the grains more accurately. Finally, the ImageJ 'Analyze Particles' routine was carried out to evaluate the grain area, perimeter and circularity $\left(4 \pi \times\right.$ area/( perimeter $\left.\left.^{2}\right)\right)$. We also attempted to utilise a circular Hough transform [179] to get the dimensions of the grains. However, this technique was ineffective for the $\mathrm{CuO}$ samples. In an image with closepacked crystallites, there is overlap and abutment between crystallites that leads to non-circular particles. The most clearly spherical particles are those that sit on top of the surface and have been able grow without interference from neighbouring crystallites. These particles are also often larger than particles within the bulk film for the same reason - they have not had to compete for space with neighbouring crystallites during the growth process. The ImageJ procedure on the other hand, handles non-circular particles more robustly, it also can present small artefacts from both the thresholding and the watershedding algorithms. This can be seen in the large spike at $\sim 5 \mathrm{~nm}$ in Figure $7.18 \mathrm{~b}$, A visual examination of the SEM image shows that there are no crystallites around $5 \mathrm{~nm}$ in size. The watershedding algorithm - which is a segmentation algorithm - reduces the size of the larger crystallites leading to an overall bias towards smaller crystallites. Despite this bias, the histogram distribution from the ImageJ is a reasonable approximation of grain size distribution in the sample (the peak is at around 23 
$\mathrm{nm}$ ). It is of particular note that the particle sizes obtained from SEM image analysis correlate closely with the average values obtained from the x-ray analysis. This implies that the observed particles are in fact single domain nanocrystals.

\section{Nanowire forest}

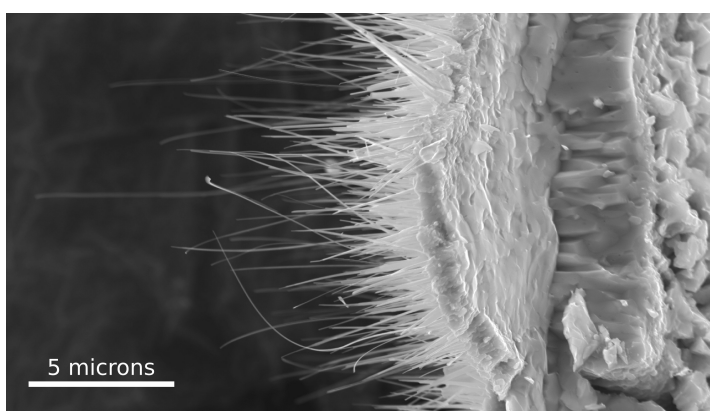

(a) View 2

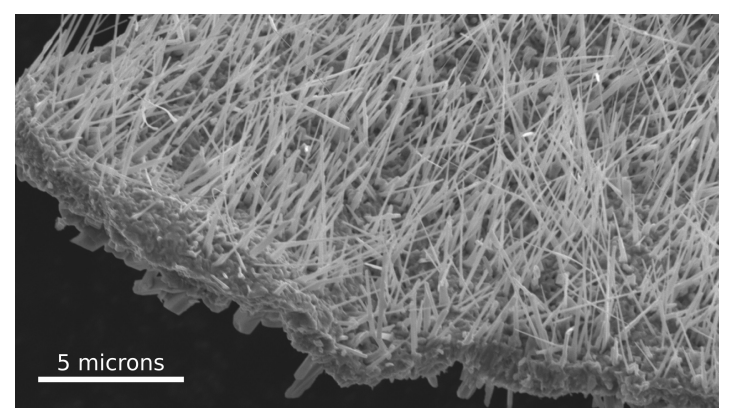

(b) View 1

Figure 7.19: Two views of a $\mathrm{CuO}$ nanowire forest prepared using the thermal oxidation technique.

Figure 7.19 shows two views of a small flake containing a growth of $\mathrm{CuO}$ nanowires. The strata seen within the flake in Figure 7.19 a follow the description provided in the literature [134]: nanowires, thin $\mathrm{CuO}$ layer and a thick, pillar-like $\mathrm{Cu}_{2} \mathrm{O}$ layer. The wires grow in a predominantly vertical direction (Figure 7.19b) but there is considerable local variation in the growth direction. Similarly, the lengths of the wires is quite variable and wires can grow to $10 \mu \mathrm{m}$ (Figure 7.19a). The growth of the nanowires depends upon feedstock of copper ions diffusing from the copper rich layers below most likely via a grain boundary diffusion process [169]. A closer inspection of some of these nanowire growth areas at a lower magnification reveal a similar hill and valley geography seen in the thin films (Figure 7.20). The hill features have no nanowire growth while the valleys flourish with varying degrees of nanowire coverage. The non-uniformity of coverage is a complex problem beginning with the still unknown issue of areas where nanowire nucleation is favourable. From there, the growth at a local level is complicated by air flow fields and the flux of copper ions diffusing to the surface.

The macroscale coverage of $\mathrm{CuO}$ nanowires is visually discernible by a velvety, matte black coverage (Figure 7.11). When the entire substrate is covered, the stratified layers seen in Figure 7.19 a are unadhered to the substrate. From observation, the peeling of the oxide layers from the underlying copper button seems to happen while the sample is cooling within the furnace. Slower cooling rates do not appear to reduce flakiness indicating that the intrinsic adhesion between the pillar-like $\mathrm{Cu}_{2} \mathrm{O}$ layer and the copper is very poor. However, the advantage of this mechanism is the reusibility of the copper button for subsequent growths. For the purposes of Raman measurements, the flaking was of little concern. 


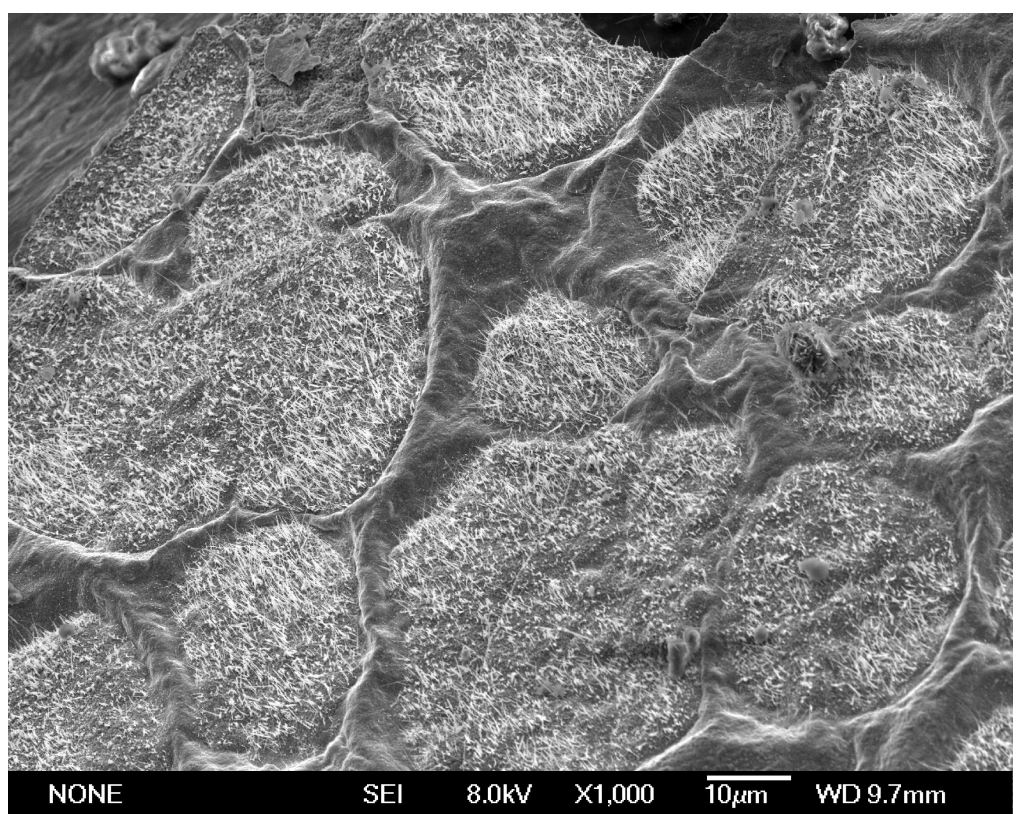

Figure 7.20: Hill and valley topology on a flake containing nanowires. 


\section{Chapter 8}

\section{Raman spectroscopy of $\mathrm{CuO}$ thin films}

\subsection{Introduction}

Raman studies on bulk $\mathrm{CuO}$ have been largely focused on forging a connection between $\mathrm{CuO}$ and the high temperature superconducting (HTS) cuprates. $\mathrm{CuO}$ was used as a simpler model system for exploring superconductivity in the more complex cuprates. Raman spectroscopy has been an important aspect of these investigations and has been used to identify structural [145, 136], magnetic [145] and spin-phonon [158] -related effects in $\mathrm{CuO}$ that could be linked back to the HTS cuprates in their superconducting state. As a result, the main Raman modes in $\mathrm{CuO}$ have been well studied at low temperature.

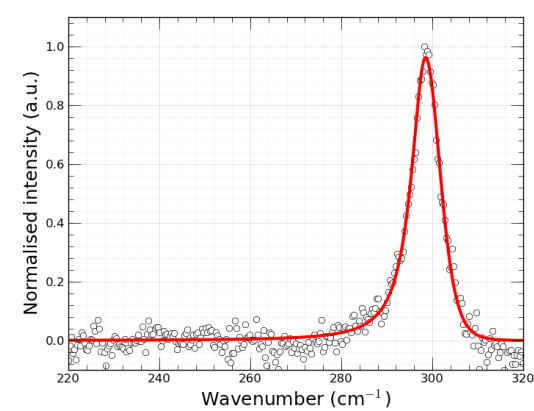

(a) Sintered pellet

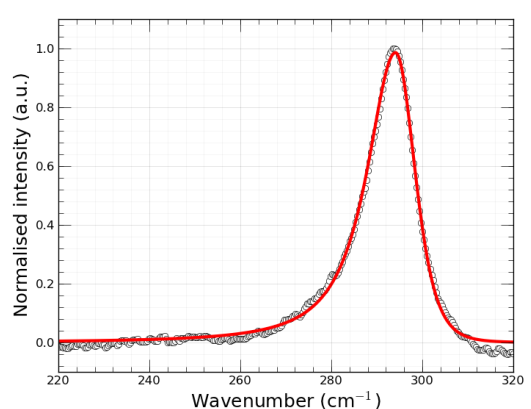

(b) Thin film

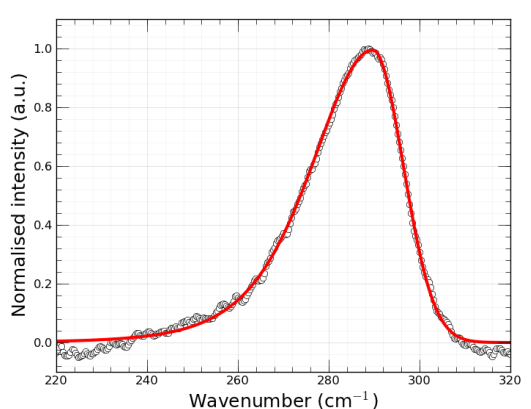

(c) Nanowire forest

Figure 8.1: Full power spectra from three morphologies of $\mathrm{CuO}$.

More recently, nanostructured forms of $\mathrm{CuO}$ (nanocrystals and nanowires) have been studied using Raman spectroscopy in an effort to understand quantum confinement phenomena [148, 150, 149, 151]. However, both these branches of studies have left the study of high temperature anharmonicity for the Raman modes untouched. This behaviour is particularly important for the understanding and deconvolution of local heating effects from the interesting confinement phenomena. The significance of local heating in nanostructures with quantised dimensions close to or below the exciton Bohr radius has been demonstrated by several groups for a number of materials (see Chapter 7.3.2). 
Heating effects due to morphological variation are significant in $\mathrm{CuO}$. Figure 8.1 shows the Raman lineshape (of the $A_{1 g}$ mode) for three different structures of $\mathrm{CuO}$ : a sintered pellet (bulk approximation), nanocrystalline thin film and a nanowire forest. Neither of the two nanostructures have dimensions within the quantum confinement regime so the visible changes to the lineshape are due to other physical effects. This chapter and the following chapter will demonstrate that the changes in the Raman lineshape of $\mathrm{CuO}$ nanomaterials are entirely due to laser heating effects.

This chapter discusses the lineshape of the nanocrystalline thin film alone. The first part presents the temperature dependence of the $A_{1 g}$ Raman mode between 200 and 700 K. From this, we can formulate a semi-empirical equation that can fully describe the Raman lineshape (intensity, peak position and linewidth). The second part of the chapter illustrates the use of local heating to gain several physical insights. The unique morphology of the thin film allows for the sampling of Raman spectra from $300 \mathrm{~K}$ to temperatures $300 \mathrm{~K}$ greater than that probed using a sample heating stage. The behaviour of the Raman lineshape under local heating is shown to be equivalent to ambient heating and the model describing anharmonicity is found to hold to $1000 \mathrm{~K}$.

The high absorption coefficient of $\mathrm{CuO}$ for laser energies in the visible spectrum combined with the low conversion to Raman photons (non-resonant Raman since $\mathrm{E}_{\text {direct }}>2.5 \mathrm{eV}$ ) means a significant portion of the absorbed energy is converted to heat. This combined with a low thermal conductivity leads to the sample heating to $1000 \mathrm{~K}$ under a $\sim 5-6.7 \mathrm{~mW}$ He-Ne laser $\left(\mathrm{E}_{\text {laser }}=1.96 \mathrm{eV}\right)$. By increasing the laser energy by $0.5 \mathrm{eV}$ to $2.41 \mathrm{eV}$ using a $\sim 12 \mathrm{~mW} \mathrm{Ar}^{+}$ ion laser, the increased absorption in $\mathrm{CuO}$ leads to local temperatures that take the material through a phase transition to $\mathrm{Cu}_{2} \mathrm{O}$.

These studies of extreme heating in $\mathrm{CuO}$ show why it has been possible for synthetic chemists to reduce $\mathrm{CuO}$ nanoparticles to copper using a lab laser with $\lambda=532 \mathrm{~nm}$ [180]. They also illustrate that any studies intent on probing confinement effects in $\mathrm{CuO}$ must ensure that heating effects are either accounted for in the experimental conditions or in the model used to describe the data. Neither of these suggestions have been utilised so far and the conclusions of these studies are called into question with our results.

\subsection{Temperature dependence of $\mathrm{CuO}$}

Temperature-dependent spectra were collected between $200-700 \mathrm{~K}$ at $\sim 500 \mu \mathrm{W}$ with an $\mathrm{N}_{2}$ cooled cryostat. Care was taken to ensure that these spectra were collected from a place on the sample with uniform morphology. This was done by refocusing the laser beam and checking the 
morphology using the attached optical microscope prior to every measurement. The $100 \times 0.6$ NA objective was used with the cryostat and has a beam waist of approximately $600 \mathrm{~nm}$ (measured using the method from [24] outlined in Section 2.2.2). The $A_{1 g}$ peak was best fit using a pseudo-voigt lineshape as shown in Eq. 8.1 [181. This lineshape is a simple approximation of the Voigt lineshape where the contribution from the lorentzian and gaussian components are convoluted. The pseudo-Voigt lineshape allows both components to be mixed additively with a mixing parameter, $\eta$, that varies between 0 (pure gaussian) and 1 (pure lorentzian). The mixing parameter was found to be temperature-independent (over the range of temperatures measured) with an average value of 0.5 . With this parameter held constant, only the peak position $\left(\omega_{\text {peak }}\right)$ and linewidth or full-width at half-maximum (FWHM, $\Gamma$ ) were fitted for all spectra.

$$
I\left(\omega_{\text {peak }}, \Gamma\right)=I_{0} \quad \eta \frac{(\Gamma / 2)^{2}}{\left[\omega-\omega_{\text {peak }}\right]^{2}+(\Gamma / 2)^{2}}+(1-\eta) \quad \exp \frac{\left(\omega-\omega_{\text {peak }}\right)^{2}}{\Gamma^{2} / 4 \ln (2)}
$$

The temperature dependence of each of the parameters that describe the Raman lineshape are detailed in the following two sections beginning with the peak position and linewidth. The second section describes the dependence of the Stokes intensity and signal as a function of temperature. We find this latter parameter to follow a unique temperature dependence and suggest a possible physical mechanism that leads to this observation. Most importantly, the temperature dependence of the Raman signal allows us to model a semi-empirical formulation describing the full Raman lineshape at any given temperature within $300-\sim 1000 \mathrm{~K}$. This formulation becomes the bedrock for understanding the asymmetry seen in the Raman lineshape of the nanowire forests (Figure 8.1c).

\subsubsection{Peak position and linewidth}

Figure 8.3 shows the $A_{1 g}$ mode red-shifting and broadening with increasing temperature. The change in $\omega_{\text {peak }}$ can be explained by a combination of two anharmonic effects (Chapter 2.1.1): volume effects like thermal expansion and phonon decay processes. The broadening of the peak however is due to phonon decay processes alone and is proportional to the inverse phonon lifetime, $\tau^{-1}$. In order to calculate the contribution of thermal expansion to the peak shift, we use the equation $\left.\Delta \omega_{\text {thermal }}=\omega_{0}[\exp (3 \gamma \alpha T)-1]\right)$ [166]. The parameters $\gamma$ and $\alpha$ are the Grüneisen parameter and the linear thermal expansion coefficient respectively. This equation is an approximation of the full equation which includes the temperature dependence of $\alpha$. The value of $\alpha$ from Ohashi et al. at $300 \mathrm{~K}$ is an approximate average for the temperature dependence of $\alpha(T)$ between $300 \mathrm{~K}$ and $700 \mathrm{~K}$ [156]. Using the values: $\gamma=0.37$ and $\alpha=6.0 \times 10^{-6}$ $\mathrm{K}^{-1}$ [182], the contribution of the red-shift from thermal expansion at $673 \mathrm{~K}$ can be calculated 


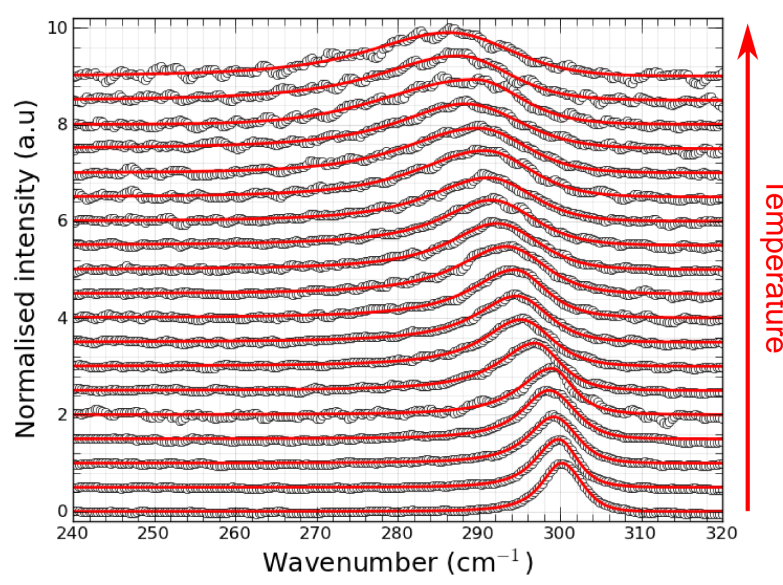

(a) Temperature dependence

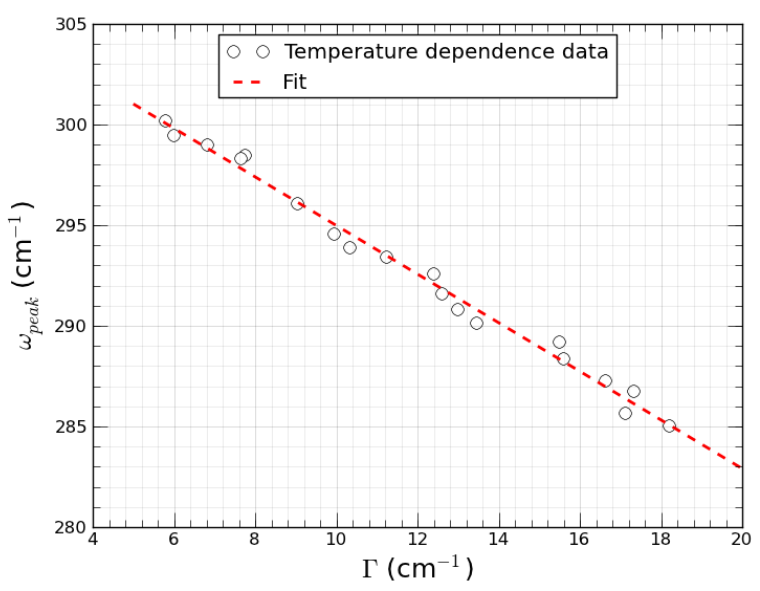

(b) Position vs. linewidth

Figure 8.2: (a) Water fall plot of temperature dependence spectra showing redshifting of the $A_{1 g}$ peak position and broadening of the peak linewidth with increasing temperature. (b) Peak position vs. linewidth for the $A_{1 g}$ peak across different temperatures.

as $1.4 \mathrm{~cm}^{-1}$. This red-shift is far lower than the $18 \mathrm{~cm}^{-1}$ shift measured in $\omega_{\text {peak }}$ at $673 \mathrm{~K}$, suggesting that the position shift can also be described by phonon decay alone. However, the convenience of this calculation is complicated by the fact that the volume Grüneisen parameter for the crystal can be different from that of a given vibrational mode (Chapter 2.1.1). Hence, we have to combine the mode Grüneisen parameter for the $A_{1 g}$ mode from a study by Reimann et al. [136] $(\gamma=0.86)$ along with the thermal expansion coefficient given above. Since this more accurate value of $\gamma$ is only twice the value of that from Ohashi et. al, the thermal expansion contribution to the redshift comes out to be $3.2 \mathrm{~cm}^{-1}$. Since more than $80 \%$ of the observed redshift at $673 \mathrm{~K}$ is due to phonon decay processes, we can be reasonably confident in our assumption to ignore thermal expansion effects in the peak frequency shift of the $A_{1 g}$ mode of $\mathrm{CuO}$.

As described earlier in Chapter 2.1.1, there are two general models that describe phonon decay channels in materials. The model of Klemens [8] describes the decay of an optical phonon into two symmetric acoustic phonons: the so-called 'three phonon decay'. This model was later extended with an additional term accounting for 'four phonon decay' by Balkanski et al. [11]. In $\mathrm{CuO}$, we have chosen to fit the data with the Klemens model alone. As described in Chapter 2.1.1, the relationship between $\omega_{\text {peak }}$ and $\Gamma$ is linear when the two parameters follow the same model (Figure 8.2b). The lack of thermal expansion effects means that $\omega_{\text {peak }}(T)$ is also described by phonon decay processes; either three or four phonon. However, there is no apparent non-linearity in the data of either $\omega_{\text {peak }}(T)$ or $\Gamma(T)$ (Figure 8.3) to require the model of Balkanski et al.

From the large linewidth of $30 \mathrm{~cm}^{-1}$ at $1000 \mathrm{~K}$, the phonon lifetime can be calculated to be 0.35 ps. A short lifetime is likely due to a large number of available decay channels. Neutron 
scattering experiments of the phonons in $\mathrm{CuO}$ by Reichdart et al. [163] show there to be around 5 -6 branches with phonons of frequency $\omega_{0} / 2$ available for coupling with the $A_{1 g}$ phonon. By comparison, silicon [183] and germanium [184] have only one available phonon branch and consequently much smaller linewidths $\left(\sim 12 \mathrm{~cm}^{-1}\right)$ at $1000 \mathrm{~K}[13$.

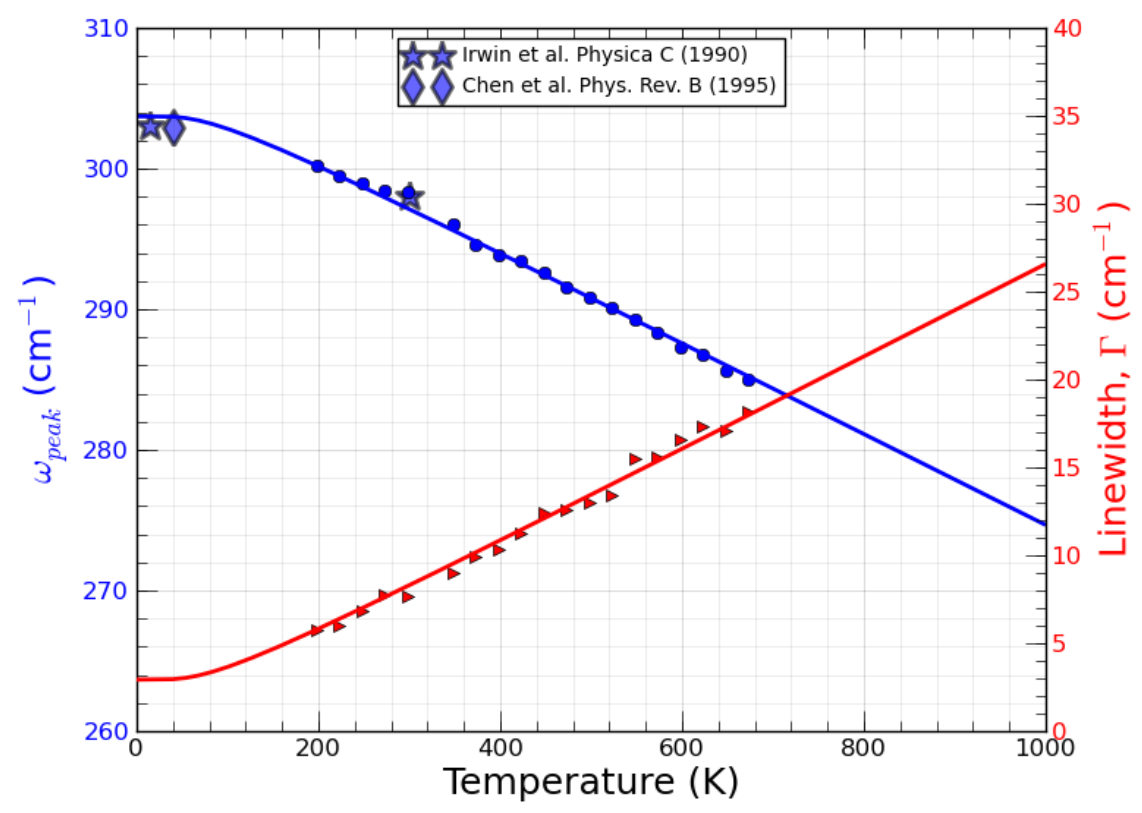

Figure 8.3: Calibration of peak position and linewidth with temperature.

An early study on sintered $\mathrm{CuO}$ found the value of $\omega_{\text {peak }}$ to be $303 \mathrm{~cm}^{-1}$ at $15 \mathrm{~K}$ [144]. Due to the lack of low temperature data from our experiments, we have included both this value and a second value at $40 \mathrm{~K}$ [158] in our peak frequency dataset in order to fit $\omega_{\text {peak }}(T)$. This composite dataset was then fit using the Klemens model (Eq. 2.9) and is shown in Figure 8.3. The limiting peak frequency as $\mathrm{T} \rightarrow 0 \mathrm{~K}$ is $303.7 \mathrm{~cm}^{-1}$; very close to the literature value of 303 $\mathrm{cm}^{-1}$ at $15 \mathrm{~K}$. The temperature dependence of the linewidth was fitted using our data alone and the limiting linewidth (as $\mathrm{T} \rightarrow 0 \mathrm{~K}$ ) of $2.9 \mathrm{~cm}^{-1}$ matches the value of $2.88 \mathrm{~cm}^{-1}$ - obtained from the linear relationship between the peak frequency $\left(303.7 \mathrm{~cm}^{-1}\right)$ and linewidth. The fitted values of $\omega_{0}, A_{\omega}$ and $A_{\Gamma}$ are given to 4 s.f. as $307.3 \mathrm{~cm}^{-1},-3.598 \mathrm{~cm}^{-1}$ and $2.928 \mathrm{~cm}^{-1}$ respectively.

Previous studies have noted that the peak position can be affected by intrinsic stress in the sample [185] and stress induced by heating [186]. Our sample has been shown to be free of intrinsic stress from Williamson-Hall analysis of the dominant XRD peaks [187]. The effects of heat-induced stress can also be deemed negligible due to the sample morphology and absorbance. Signal is only collected from within $\sim 50 \mathrm{~nm}$ of the top surface due to the high absorption coefficient of $\mathrm{CuO}$ (around $10^{5} \mathrm{~cm}^{-1}$ at $1.96 \mathrm{eV}$ [22, 23]). This top layer is comprised of grains that have some capability to expand (Figure 7.17b) suggesting expansion-related stresses will be minimal. 


\subsubsection{Peak maximum and integrated intensities}

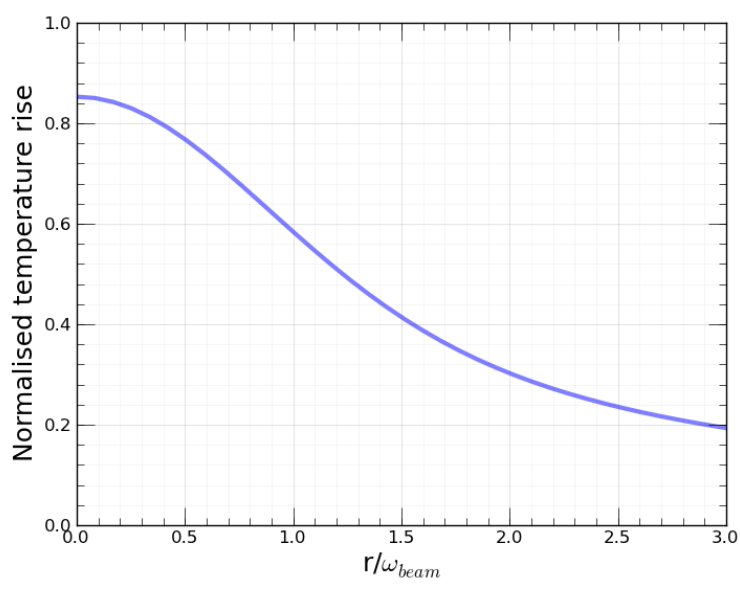

(a) Calculation of peak temperature

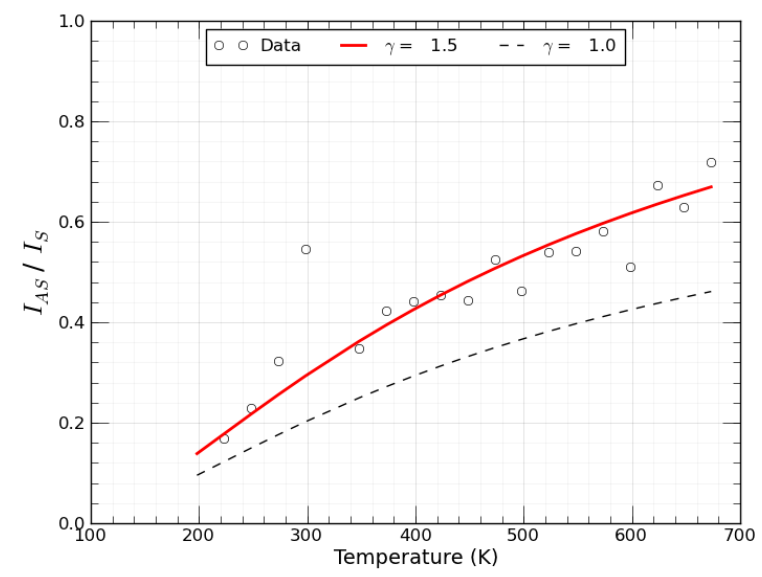

(b) $I_{A S} / I_{S}$

Figure 8.4: (a) Calculation of normalised temperature rise using the analytical formultion of Lax. (b) Anti-Stokes to Stokes intensity ratio as a function of temperature.

The Anti-Stokes to Stokes intensity ratio $\left(I_{A S} / I_{S}\right)$ is the simplest way to estimate local temperature using Raman spectroscopy as it doesnt require any calibration. In the non-resonant case, the effective temperature can be described using Eq. 2.13 (taken from [188]) with $\gamma=1.0$. However, the use of $I_{A S} / I_{S}$ as an accurate temperature proxy is limited to certain situations: homogeneous heating and where the Stokes and Anti-Stokes wavelengths have similar detector efficiencies. Homogeneous temperature of the sample can be achieved when the entire sample is heated, or when the thermal conductivity is high enough that a gaussian intensity distribution on the sample can be converted to a uniform temperature within the acquisition time. In the case of non-uniform local heating with a gaussian laser beam, the actual temperature rise can be as high as twice the temperature estimated from $I_{A S} / I_{S}$ [16]. In $\mathrm{CuO}$, the correction factor is predicted to be 2.0 since $\omega_{0}=307.3 \mathrm{~cm}^{-1}$ (Chapter 2.1.1). Using the analytical solution of Lax et al. we can calculate the normalised temperature rise under a gaussian laser beam for $\mathrm{CuO}$ (Figure 8.4a). If we assume that light is collected from a region with a radius of $\sim 2 \omega_{\text {beam }}$, the temperature rise at the beam center is around 1.5-2.0 times higher the temperature at the edge of the collection region (uniform temperature). This calculation corroborates very well with that of Herman because $\mathrm{CuO}$ obeys one of the key assumptions used in the calculation of both Herman and Lax; i.e. high sample absorption. It should be noted that both these calculations are for the case of $t=0 \mathrm{~s}$. In a real experiment, the acquisition time might be sufficiently long to allow for cooling by conduction and the curve shown in Figure 8.4a would be considerably flatter.

While the Anti-Stokes to Stokes intensity ratio can be shown to be an unsuitable temperature proxy for laser heating by theoretical means, the observed $I_{A S} / I_{S}$ itself is different to Eq. $2.13(\gamma=1)$ for this temperature-dependent study; which is considered homogeneous heat- 
ing since laser power was quite low. A correction factor of 1.5 is required to fit the observed data. This correction could be due to either different efficiencies for the Stokes and Anti-Stokes photons, or a change in the optical properties of $\mathrm{CuO}$ with temperature. The difference in detection efficiency for Stokes and Anti-Stokes photons is only 3\% (Figure 2.3a) and does not account for the $50 \%$ increase seen in Figure 8.4b. Hence, we can surmise is that the optical properties of $\mathrm{CuO}$ have a significant temperature dependence.

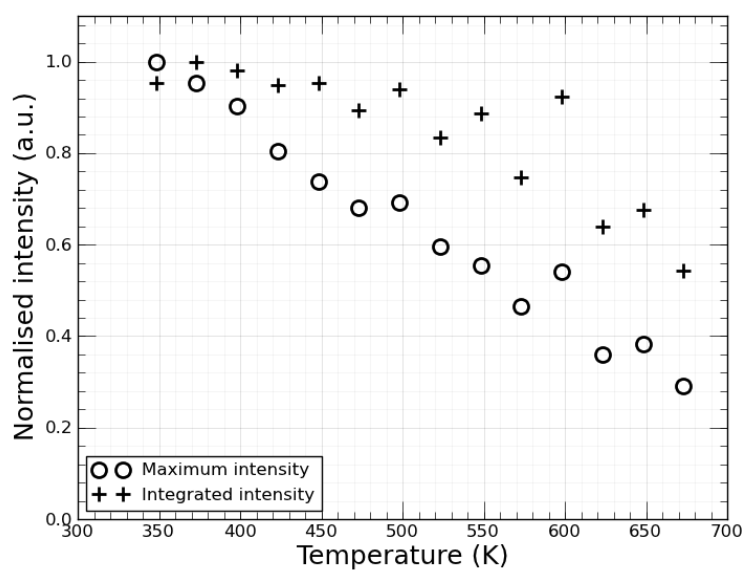

(a) Simulating Raman signal

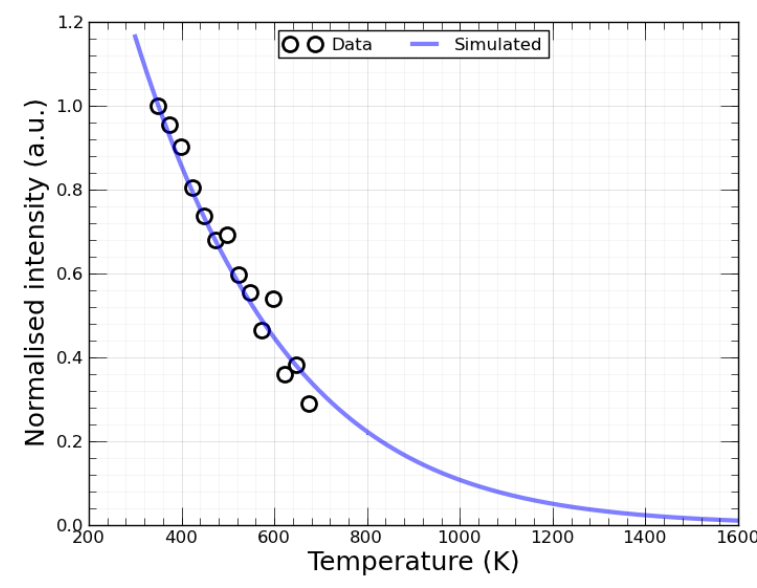

(b) Analytical approximation of $I_{S}$

Figure 8.5: (a) Observed data of the maximum Stokes intensity and integrated intensity vs. temperature.(b) Analytical approximation of Stokes intensity.

Since the temperature-dependent optical properties of $\mathrm{CuO}$ are significant enough to affect the Stokes intensities, an accurate description of the Raman lineshape must account for it. Figure $8.5 \mathrm{a}$ shows that both the Stokes intensity and integrated intensity decrease with temperature. The decrease of the Stokes peak with temperature is not a consequence of the decreasing population of ground state phonons (since the Planck distribution can be simplified as a linear increase at the high temperatures). Eq. 2.14 shows that the Stokes intensity is effectively a linear function of temperature as its major component is the Planck distribution. A correction factor appended to Eq. 2.14 gives the normalised Stokes intensity (Eq. 8.2) and can reproduce the trend in the observed data (as shown in Figure 8.5b). A linear trend can also describe the Stokes intensity data but we can show that the Raman signal will be zero by $800 \mathrm{~K}$. In local heating experiments, signal from regions with temperatures can be as high as $1000 \mathrm{~K}$ (Section 8.3.2), making a linearly decreasing trend unlikely.

$$
I_{S}=\exp \left(-4.5 \times 10^{-3} T\right) 1+\left(\exp \left(-h c \omega_{0} / k_{B} T\right)-1\right)^{-1}
$$

To explore how the Stokes intensity trend affects the Raman signal (integrated intensity), the Raman spectra between 350 and $800 \mathrm{~K}$ were simulated using the established calibrations 
for $\omega_{\text {peak }}(\mathrm{T})$ and $\Gamma(\mathrm{T})$, and the empirical linear and exponential decrease of the Stokes intensity with temperature. These values were used to simulate the Stokes lineshape (using Eq. 8.1) from which the integrated intensity was calculated for $\omega$ between 220 and $320 \mathrm{~cm}^{-1}$. The results of the simulation are shown in Figure 8.6. The close match between the trend of exponential decrease correction to the Stokes scattering rate formula with the observed data in Figure $8.6 \mathrm{~b}$ indicates that the observed decrease in Stokes intensity drives the decreasing Raman signal. This seems a trivial observation but the key here is that the trend in the Stokes intensity is lower than that expected due to a decrease in phonon population alone. The exponentially decreasing Stokes intensity with temperature is also qualitatively matched by a study on silicon (Figure 2.4b) indicating that this functional form is qualitatively appropriate.

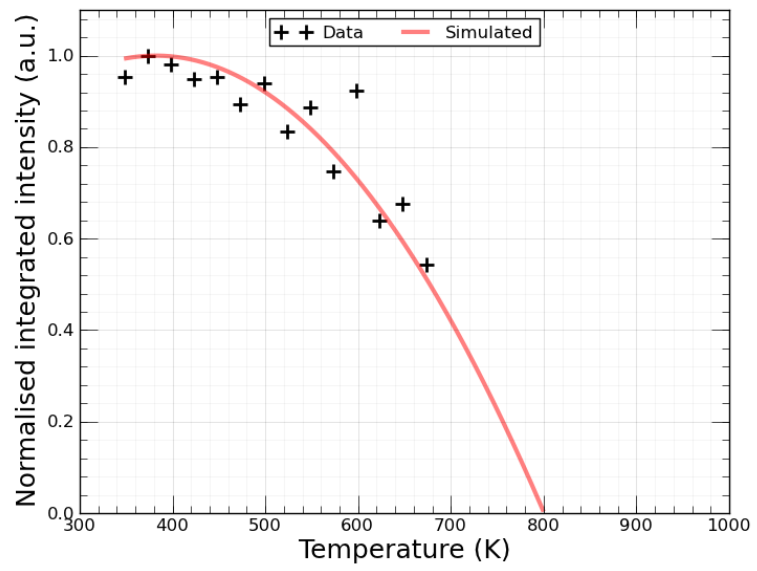

(a) Linearly decreasing Stokes intensity

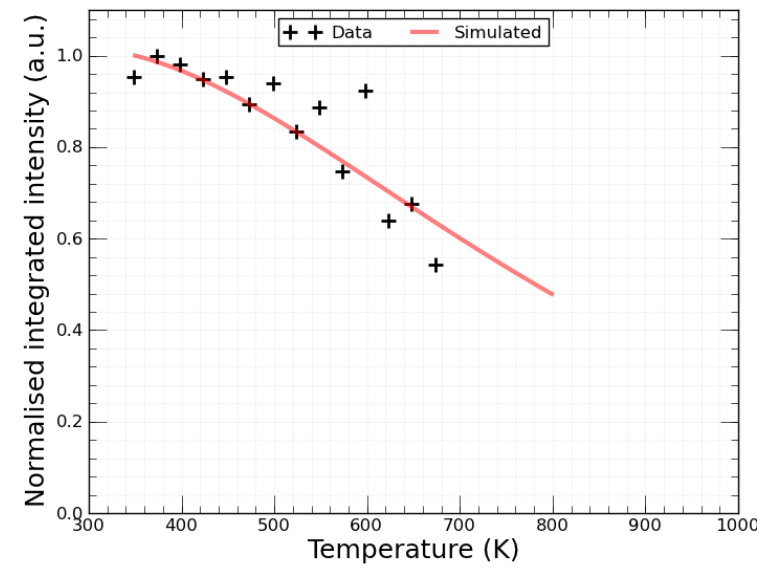

(b) Exponential decrease in Stokes intensity

Figure 8.6: Normalised integrated intensity from temperature dependence plotted against simulated data using (a) linear decrease in Stokes intensity and (b) decreasing exponential factor for Stokes intensity scattering rate formula.

In summary, we have examined in detail the temperature dependence of the $A_{1 g}$ Raman mode in $\mathrm{CuO}$ between 200 and $700 \mathrm{~K}$. We find that volume effects have very little effect on the observed temperature dependence of the peak frequency. Both the peak frequency and the linewidth can be described using the model of three phonon decay, where the optical phonon decays into two symmetric acoustic phonons. We have established a calibration curve for the peak position and linewidth using this model. We additionally find that both the maximum intensity and integrated intensity of the Stokes peak decrease with temperature. Using the calibration curve for the peak frequency and linewidth and an exponential decrease in the maximum Stokes intensity with temperature, we can fully model the Raman lineshape. The decrease in the Stokes intensity is greater than that expected from a decreasing population of ground state phonons with temperature. This is indicative of a significant temperature dependence for some fundamental optical property of cupric oxide (e.g. refractive index and absorbance). 


\subsection{Laser heating effects}

As seen in the previous section, the Raman modes of a material have a distinct temperature dependence: the peak position and linewidth of the modes red-shift and broaden with increasing temperature. Local heating by the laser beam can have a similar effect as increasing the temperature of the entire sample. We find that laser heating in $\mathrm{CuO}$ is a dramatic effect and the extent of local heating is highly dependent upon the crystallite (grain) size, sample morphology and, laser energy (both power and wavelength dependence). In this section, we investigate the above physical parameters in detail to identify unique phenomena such as (i) an equivalence between laser and ambient heating, (ii) evidence of inhomogeneous temperature distributions at sites with poor connectivity to a thermal sink and, (iii) laser-induced phase transition from $\mathrm{CuO}$ to $\mathrm{Cu}_{2} \mathrm{O}$.

\subsubsection{Pellets}

One of the first lineshape comparisons we can make is between the three samples with grains namely, the sintered pellet, unsintered pellet and the thin film. Full power spectra from the three samples was obtained from a spatial mapping experiment. Figure 8.7 shows a few of the characteristic spectra from each sample where the collected spectra were background-corrected and fitted with a pseudo-voigt lineshape as described in Section 8.2. In the sintered pellet, we see that the $A_{1 g}$ mode is narrow and centered at around $298 \mathrm{~cm}^{-1}$ while this mode is considerably broadened and red-shifted for the other two samples. In fact the red-shifted spectra from the unsintered pellet and the thin film are shifted to around $280 \mathrm{~cm}^{-1}$. This similarity can be connected to the size of the component grains within these two samples. From the Williamson-Hall analysis presented in Chapter 7.4.1, we found that the sintered pellet has a grain size of $\sim 100 \mathrm{~nm}$ while the thin film and unsintered pellet have a grain size around $30 \mathrm{~nm}$. The similarity in size between the thin film and unsintered pellet means that the laser heating effects are about the same in both. The samples are heated by the laser due to suppressed thermal conductivities. Thermal conductivity can be suppressed by boundary scattering (finite boundaries e.g. grain boundaries), increase in point defects, lattice imperfections and scattering from phonons (important at higher temperatures) [189]. In the case of the unsintered pellet and thin film, the suprression could be due to one or more of the above effects. The disappearance of this effect upon sintering suggests that the most likely mechanisms are boundary scattering (small grain size) and possible lattice imperfections (which can be corrected by high temperature annealing). As noted in the previous section, we can assume that strain-related effects are minimal given the comparative values of grain size and the depth of optical absorption. Additionally, the extent of homogeneous strain calculated from the Williamson-Hall analysis comes to around $0.5 \%$ (negligible) for both samples. 


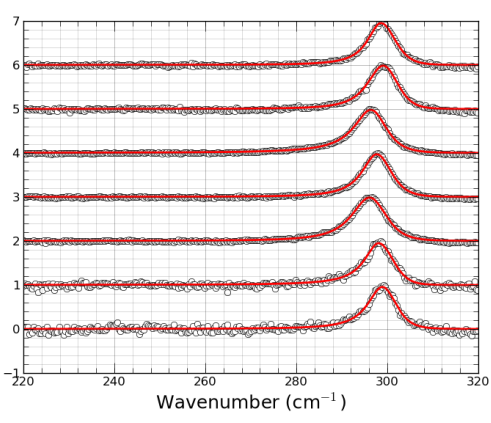

(a) Sintered pellet

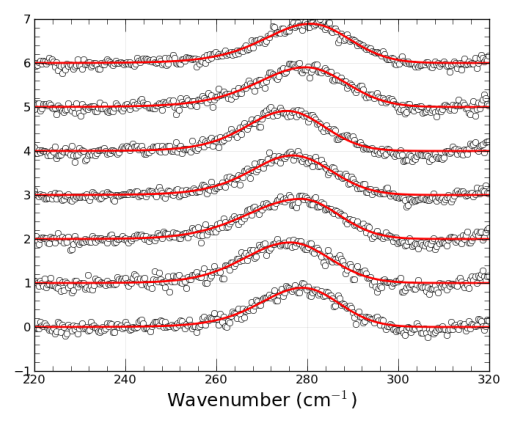

(b) Unsintered pellet

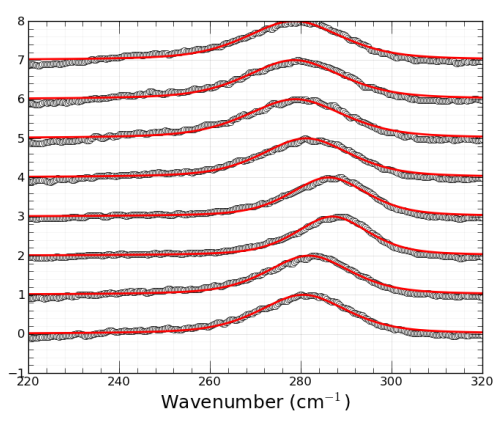

(c) Thin film

Figure 8.7: Full power spectra from three $\mathrm{CuO}$ samples.

\subsubsection{Thin films}

Characterisation of the thin film morphology in Section 7.4 .3 showed that the macroscopic landscape is comprised of hill and valley features. These two features show distinctly different Raman spectral characteristics. A simple power dependence at hill and valley sites show that the hill heats up considerably more than the valley (Figure 8.8). The clear power dependence of the peak position and linewidth indicates strong local heating by the laser as seen earlier in the previous section. At powers below $1 \mathrm{~mW}$, the peak position at $\sim 298 \mathrm{~cm}^{-1}$ is consistent with the literature value. At higher powers, $\omega_{\text {peak }}$ undergoes significant redshifts upto $\sim 16 \mathrm{~cm}^{-1}$ while the linewidth broadens from $8 \mathrm{~cm}^{-1}$ to $22 \mathrm{~cm}^{-1}$. The high optical absorption coefficient of nanocrystalline $\mathrm{CuO}$ at $1.96 \mathrm{eV}$ [22, 23] concentrates the incident light in a shallow region below the surface; corresponding to the characteristic penetration depth $(\sim 50 \mathrm{~nm})$. This local concentration of high energy photons combined with both a low conversion of incident photons to Raman scattered photons (non-resonant laser energy) and a low thermal conductivity (as typically seen when comparing bulk with sub-100 nm grains [155]) considerably heats the area under the laser spot at higher power levels. The difference in the extent of local heating for the hill and valley sites can be related to thermal anchoring and will be demonstrated in the following section. Since the thin film is comprised of many hills and valleys, every spatial mapping experiment on a thin film will sample both these topologies. The shoulder at $\sim 250$ $\mathrm{cm}^{-1}$ seen in the full power spectrum at a hill site (Figure $8.8 \mathrm{~b}$ ) will be discussed in Section 8.3.3.

\section{Equivalence between local and global heating}

Raman spatial mapping was carried out by rastering the laser across a region of $50 \mu \mathrm{m} \times 50$ $\mu \mathrm{m}$ with a step size of $\sim 5 \mu \mathrm{m}$ at high $(5-6.7 \mathrm{~mW})$ and low $(\sim 0.5 \mathrm{~mW})$ laser powers under ambient conditions. This collection of independent spectra were analysed using the same procedure as the temperature dependence data. Figure 8.9 a shows that the scatter of $\omega_{\text {peak }}$ versus $\Gamma$ for these power dependence spectra follow the same linear correlation between peak width and position as the temperature dependent study. This indicates that the power dependence is attributable to a homogeneous local temperature, which can be determined by converting 


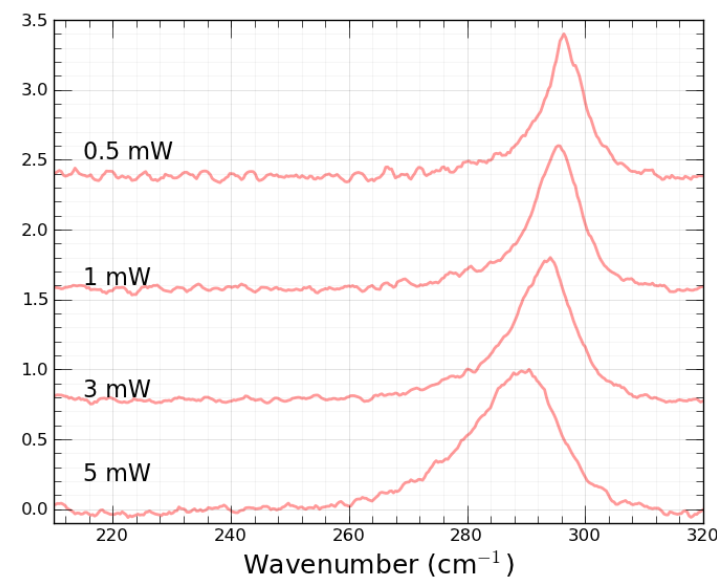

(a) Valley

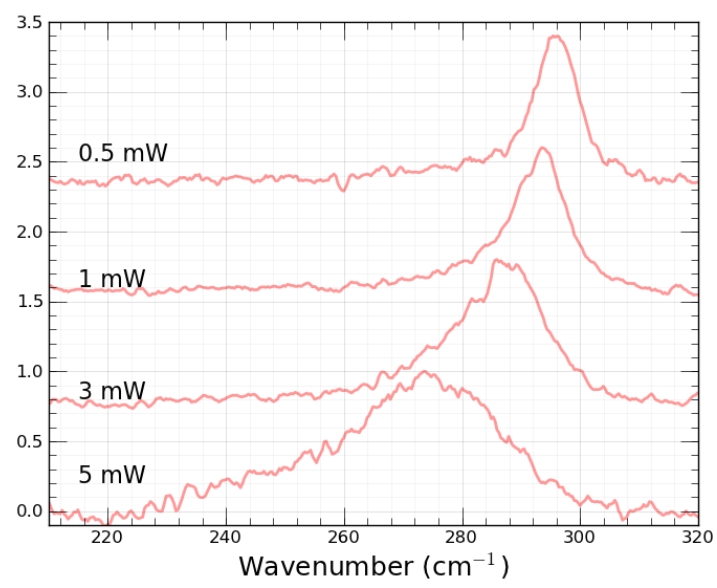

(b) Hill

Figure 8.8: Power dependence of the $A_{1 g}$ peak for two different topologies.

experimentally measured values of $\Gamma$ or $\omega_{\text {peak }}$ to a temperature using the equations described in Section 8.2. Transforming the data in Figure 8.9a into a histogram (Figure 8.9b) reveals two distinct clusters for the high power spectra; a low temperature cluster at $\sim 400 \mathrm{~K}$, and a cluster at $\sim 1000 \mathrm{~K}$ which is well in excess of the maximum temperature attainable using our external heating apparatus. This bimodal distribution can be shown to be a result of the micro-morphology of the $\mathrm{CuO}$ film, which comprises of micro-bubbles ('hills') situated upon a planar background surface ('valleys') as seen in Figure 8.9c. Spectra collected independently from larger micro-bubbles (that are visible through the optical microscope) correlate with the high temperature spectra seen in the spatial maps (Figure 8.8b).

We can demonstrate that the bimodal temperature distribution is caused by the combined effect of two major components that vary with morphology: (i) thermal coupling to the sapphire substrate; and (ii) focal intensity as a function of sample surface height. A third component, the in-plane $\mathrm{CuO}$ film thermal conductivity, can be linked to the behaviour of the sample temperature upon continual illumination but isn't necessary to understand the bimodal distribution. The contributions from the two main components can be seen by conducting an axial dependence study in which the focal depth above and below the focal plane is varied at both hill and valley sites. Negative distances indicate that the focal spot lies beneath the sample surface, i.e. within the film. The observed Raman spectra were analysed as outlined in Section 8.2.

Figure 8.10 a shows the integrated intensity of the fitted pseudo-voigt peak as a function of the axial displacement from the focal plane for a hill and valley. The data corresponding to the valley site in Figure 8.10a is effectively the same as that of silicon (or some other standard) used in a typical axial dependence measurement to obtain the confocal depth for the Raman microscope. From both the silicon (not shown here) and $\mathrm{CuO}$ axial dependence we get a confocal depth of $6 \mu \mathrm{m}$ from the full width at half maximum by fitting a lorentzian lineshape to the axial 


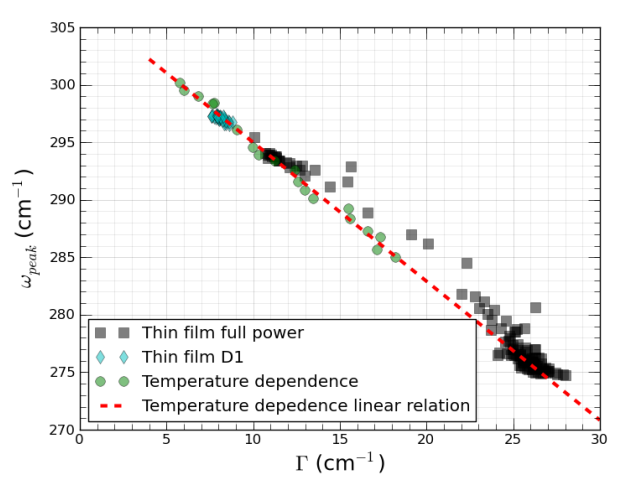

(a) Peak position vs. linewidth

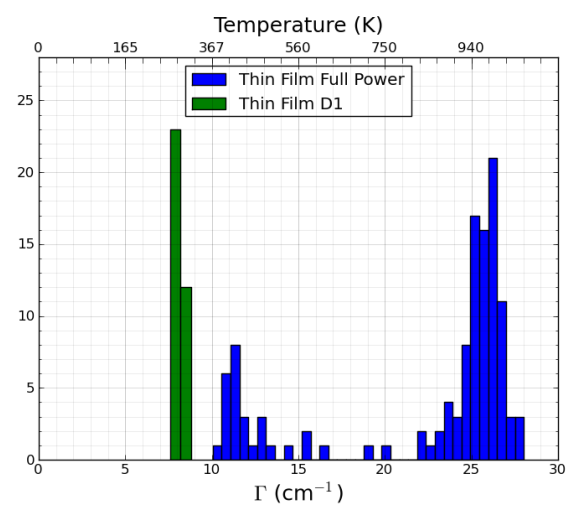

(b) Histogram of linewidths

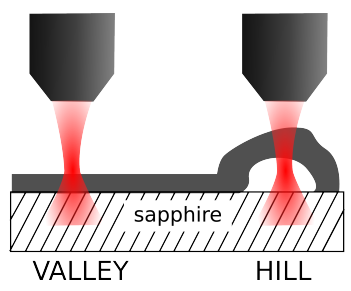

(c) Schematic of hill vs. valley

Figure 8.9: (a) and (b) Equivalence between global and local heating. (c) Schematic of a spatial mapping acquistion when intial focus is on a valley site.

dependence data since the instrumental collection profile is unlikely to be dependent on morphology. The hill site follows the same trend as the valley site everywhere except $\pm 5 \mu \mathrm{m}$ from the focal plane. Here the signal from the hill site is only $80 \%$ of the signal from the valley and is a consequence of the reduced intensity from high temperature regions as seen in Section 8.2.2.

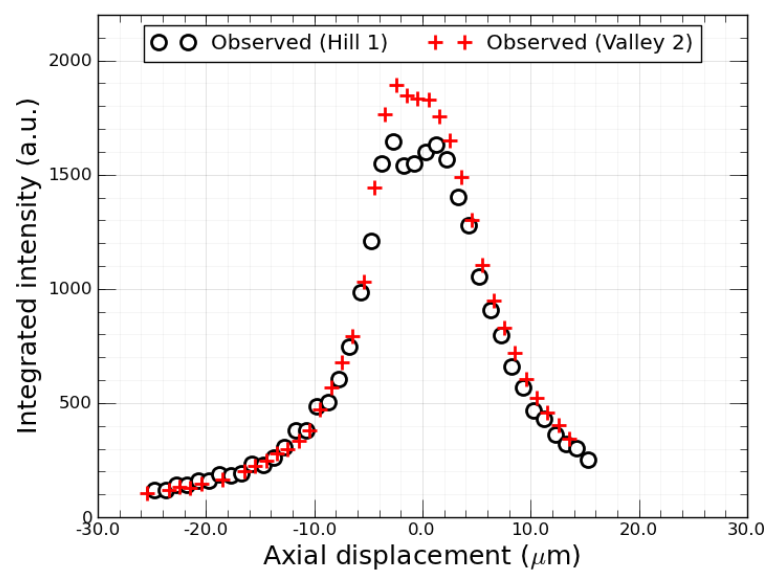

(a) Integrated intensity

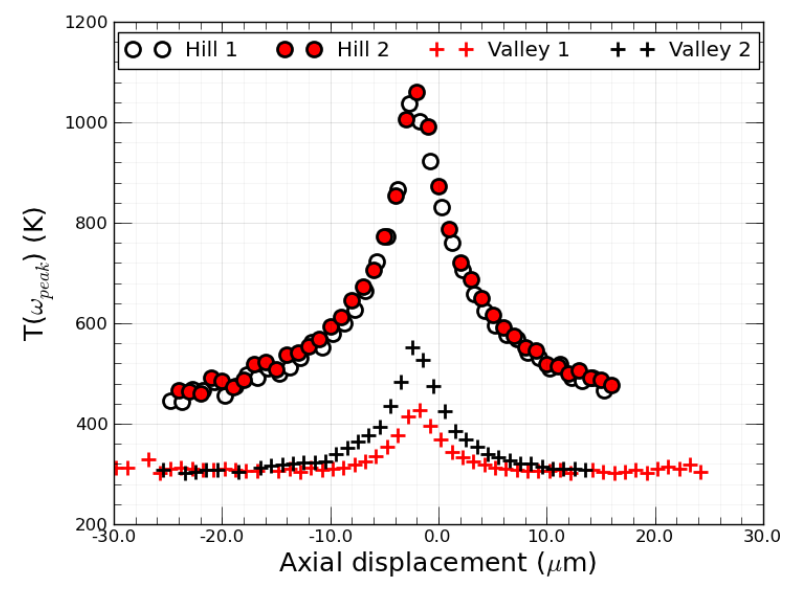

(b) Effective temperature

Figure 8.10: (a)Integrated intensity and effective temperature as a function of the axial displacement, $z$, for hills and valleys. (b) Effective temperatures at hills and valleys.

Figure $8.10 \mathrm{~b}$ shows the peak position converted to an effective temperature since it is easier to fit an accurate peak maximum for low intensity data. The variation of temperature with $z$ differs markedly between hills and valleys and implies that there is a fundamental difference in local material properties in these two regions. It is important to note that these differences are not observed at low incident laser power, hence local stress is not the cause of the differing axial dependence. Rather, the hills weaker thermal coupling to the underlying substrate due to their micro-bubble nature leads to a sharper increase in temperature with laser heating at hill 
sites than is observed in the valleys. Since the Raman mapping procedure simply rasters across the sample at a constant height (Figure 8.9c), without adjusting the focus at each acquisition point, variation in focal height will affect the laser intensity at the sample surface. The range of temperatures within the highlighted $5 \mu \mathrm{m}$ of the focal plane for the two sites, coincides with the two data clusters observed in Figure $8.9 \mathrm{~b}$. This follows from the experimental practice of focusing on a valley region at the beginning of the spatial mapping measurement. At a hill, the focal point lies within the sample and leads to considerable local heating.

The saturation temperature for the valley region corresponds to ambient while the hill still exhibits a temperature of $400 \mathrm{~K}$ at $\pm 15 \mu \mathrm{m}$ from the focus. At first glance, the saturation effect resembles an artefact of long acquisition times which can cause significant local heating. However, repeating the axial dependence measurements using a three-fold reduction in acquisition time reproduces the same data shown in Figure 8.10b. This suggests that the two saturation temperatures are indicative of a morphology dependence in the thermal conductivity, if one assumes that in-plane conduction is the dominant cooling mechanism. Of course, this assumption is only valid for lower effective temperatures (away from focus) as radiative cooling becomes significant for high temperature regions (near focus). However, with continual illumination, the local temperature cannot cool fast enough by dissipating heat through thermal conduction, leading to higher saturation temperatures even at low laser intensity.

Both the peak position and linewidth of the $A_{1 g}$ peak follow the linear relation established in Section 8.2 for global heating. This trend suggests that the integrated intensity should also follow the decreasing trend with temperature observed in the globally heated sample. Figure 8.10a shows that the hill site at $1000 \mathrm{~K}$ has a signal of $80 \%$ compared to that from the valley site at $400 \mathrm{~K}$. From Figure $8.6 \mathrm{~b}$ we see that the signal from a region at $1000 \mathrm{~K}$ should be $30 \%$ compared to a region at $400 \mathrm{~K}$. Part of the reason for the higher signal in Figure $8.10 \mathrm{a}$ can be attributed to a larger signal volume for the axial dependence data.

In summary, we confirm from power and axial dependence studies that the local heating at hill sites is due to a disconnect in the thermal anchoring to the substrate. This topological variation in the extent of local heating allows for the extrapolation of the established temperature dependent behaviour for the $A_{1 g}$ mode (Section 8.2 to $1000 \mathrm{~K}$. The continuation of the linear trend observed in Figure $8.2 \mathrm{~b}$ provides strong evidence that local heating in $\mathrm{CuO}$ causes the same change to the peak linewidth and position as changing the global temperature.

\subsubsection{Extreme heating}

In the previous section, we saw that hill sites were far more susceptible to local heating when the laser beam was close to focus since they are thermally disconnected from the sapphire substrate. From the peak positions, we could identify a maximum temperature of $1000 \mathrm{~K}$ when 
excited with a $632.8 \mathrm{~nm}$ laser. When the sample is probed with a laser wavelength of 514.5 $\mathrm{nm}$, the induced local heating is high enough for a phase transition to $\mathrm{Cu}_{2} \mathrm{O}$. Figure 8.11 shows one scenario of an extreme heating effect in $\mathrm{CuO}$. Here, a hill and valley site are independently probed with a full power $(11 \mathrm{~mW}) \mathrm{Ar}^{+}$laser with $\lambda=514.5 \mathrm{~nm}$. The thermally isolated hill site has undergone a complete transition to $\mathrm{Cu}_{2} \mathrm{O}$ while the valley site shows that only part of the probed region has been changed to $\mathrm{Cu}_{2} \mathrm{O}$. The Raman peaks associated with $\mathrm{Cu}_{2} \mathrm{O}$ are marked with a dashed maroon line while the $\mathrm{CuO}$ Raman peaks are contained within the highlighted grey region. The Raman signal of $\mathrm{Cu}_{2} \mathrm{O}$ picks out many of the vibrational modes of the crystal and is comprised of band modes $\left(\sim 100 \mathrm{~cm}^{-1}\right)$, IR-active modes $\left(\sim 150 \mathrm{~cm}^{-1}\right)$, multi-phonon modes $\left(418 \mathrm{~cm}^{-1}\right)$ and defect modes $\left(\sim 220 \mathrm{~cm}^{-1}\right)$ [190] unlike the nominally Raman-active modes of $\mathrm{CuO}$.

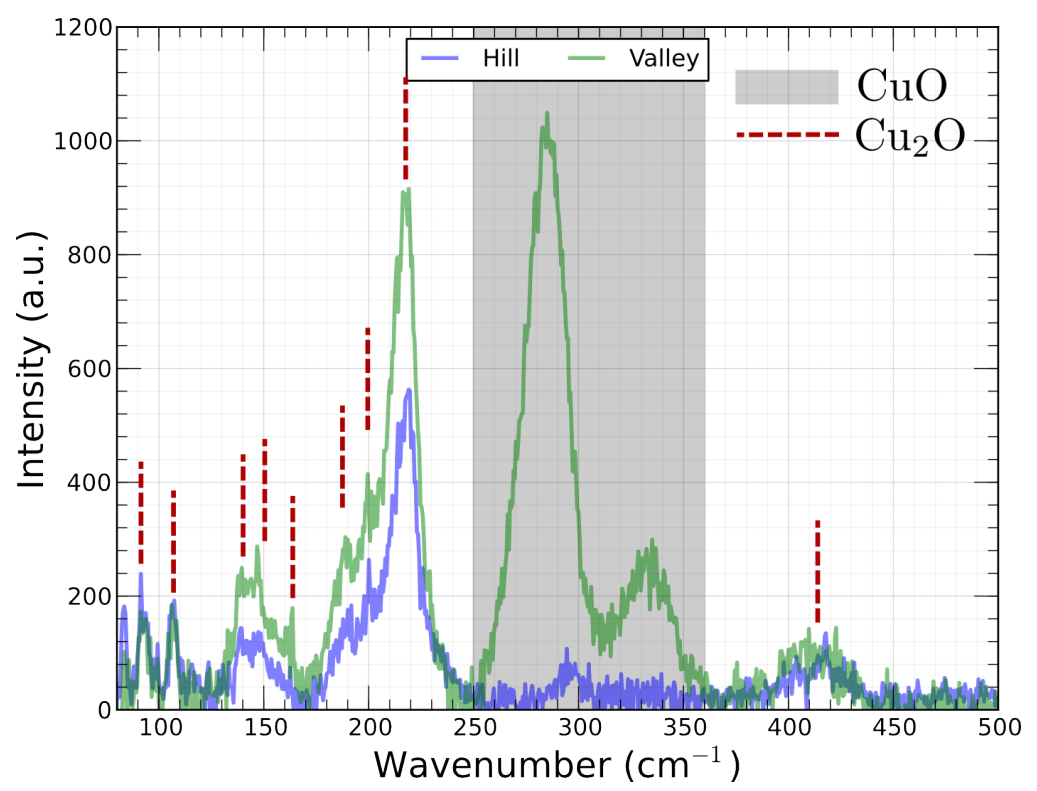

Figure 8.11: Extreme heating of a hill and valley region under $514.5 \mathrm{~nm}$ excitation.

Figure 8.11 shows that the relative amount of sample that is converted from $\mathrm{CuO} \rightarrow \mathrm{Cu}_{2} \mathrm{O}$ is dependent upon the extent of local heating. At the valley site - which is more thermally stable - we see only a partial transformation to $\mathrm{Cu}_{2} \mathrm{O}$. The $\mathrm{Cu}_{2} \mathrm{O}$ signal most likely comes from the hottest regions, e.g. directly under the laser hotspot. Regions further away from the hotspot are not hot enough to be transformed into $\mathrm{Cu}_{2} \mathrm{O}$. At the hill site, the suppression of thermal conductivity for most of the probed regions affects a nearly $100 \%$ transformation to $\mathrm{Cu}_{2} \mathrm{O}$.

The scenario described here is very similar to that observed in silicon by Kouteva-Aguireva et al. [186]. In this paper, the authors describe the Raman spectrum of silicon using a 200 $\mathrm{mW} \mathrm{CO}_{2}$ laser. While SEM images show a pit (corresponding to local melting), the authors were still able to observe a Raman peak. The conclusion was that the silicon melt was a small localised region directly under the laser hotspot surrounded by regions at considerably lower temperatures and these 'wing' regions were observed in the Raman signal. 
This picture of a very hot region and a uniform region is in good agreement with the laser heating plot shown in Figure 8.4a. The temperature rise at the hotspot at a hill site is likely to be considerably more than the plotted amount since thermal conductivity decreases with increasing temperature. In $\mathrm{CuO}$, this constriction of heat flow from the hot regions appears to be significantly large leading to a phase transition that is expected to be at $1400 \mathrm{~K}$ at an $\mathrm{O}_{2}$ partial pressure of $160 \mathrm{~mm} \mathrm{Hg}$ (atmospheric, Figure 7.1a).

Using this model of local heating, we can re-examine the interesting lineshape of the $A_{1 g}$ mode close to focus at a hill site (Figure 8.12a). The plots of a valley site at the same distance from focus is also shown for comparison. The left hand side of the $A_{1 g}$ peak from a hill shows a unique asymmetry with a jutting shoulder around $250 \mathrm{~cm}^{-1}$. From previously published work, two scenarios are possible: (i) asymmetric temperature distribution close to the hotspot that abruptly stops at the melt zone or, (ii) a structural transition in $\mathrm{CuO}$ that considerably softens the $A_{1 g}$ mode.

For the first scenario, the shape of the asymmetry on the low wavenumber side is indicative of lower signal from regions at high temperatures. In Section 8.2.2, we found that Raman intensities and signal decrease with increasing temperature. We can then picture an inhomogeneous temperature region between the hotspot and the region of uniform temperature. The observed Raman signal then is a linear superposition from all these regions. The following chapter expands on this idea but here we are only interested in a qualitative description. If we assume that the temperature and peak frequency are related by the same Klemens process discussed earlier, the shoulder at around $250 \mathrm{~cm}^{-1}$ would correspond to a temperature around $1600 \mathrm{~K}$. The top plot in Figure 8.12c shows that the asymmetric low frequency tail is comprised of Raman signal from $\mathrm{T}_{\text {uniform }}(1000 \mathrm{~K}$ in this case) to $1600 \mathrm{~K}$. An abrupt cessation of signal when the crystal loses its structure and becomes a melt could lead to the shoulder at $250 \mathrm{~cm}^{-1}$.

A high temperature XRD study on $\mathrm{CuO}$ [156] provides evidence for an alternative scenario of a structural change. From Rietveld analysis of XRD patterns, the authors found that the bending angles of the $\mathrm{Cu}-\mathrm{O}$ ladder is different below $800 \mathrm{~K}$ and that there is a significant change to the equilibrium positions of the $\mathrm{O}^{2-}$ ions at $800 \mathrm{~K}$. Since these two physical changes affect the $A_{1 g}$ mode vibration, the authors concluded the study with a comment that is likely to be a significant softening of the $A_{1 g}$ mode at $800 \mathrm{~K}$. The deconvolution of the $A_{1 g}$ peak then is a simple case of two sub-peaks: one at $\mathrm{T}_{\text {uniform }}$ of $\sim 1000 \mathrm{~K}$ and the softened peak due to temperatures above $1000 \mathrm{~K}$ at $250 \mathrm{~cm}^{-1}$ (bottom plot in Figure 8.12c). At this stage, the case for a structural change requires further study. Raman spectra of homogeneously heated $\mathrm{CuO}$ are required to at least $800 \mathrm{~K}$. Instead, we favour the model of inhomogenous heating. In the following chapter, we demonstrate a model of inhomogeneous heating based on the temperature dependence of peak position, linewidth and intensity identified in Section 8.2 . 

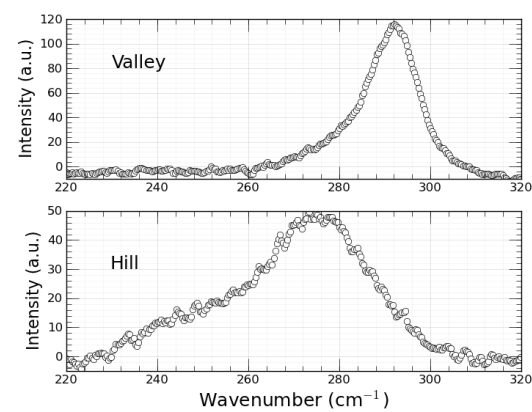

(a) Hill vs. Valley at $2.5 \mu \mathrm{m}$ into sample

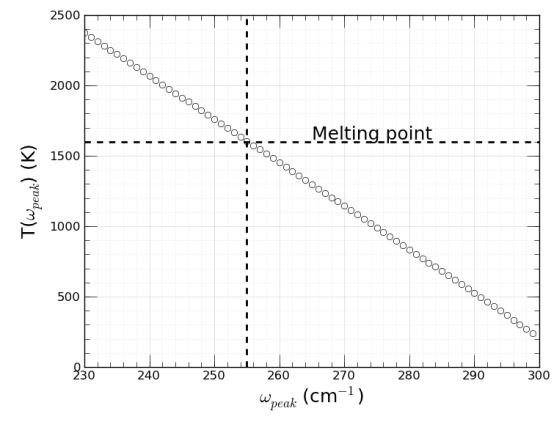

(b) Temperature vs. $\omega_{\text {peak }}$

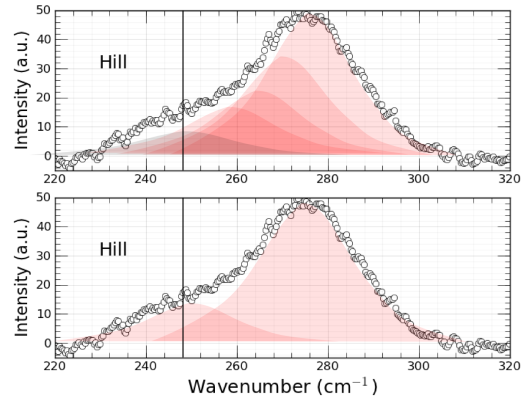

(c) Two scenarios

Figure 8.12: (a) Full power spectrum $2.5 \mu \mathrm{m}$ below the focal plane for a hill and valley. (b) Temperature (generated from peak position) vs. peak position. (c) A visual deconvolution of the observed $A_{1 g}$ lineshape at a hill site due to a linear combination of signal from regions at different temperatures (top) or a structural transition for the inhomogeneous temperature region at the laser hotspot (bottom).

The unusual $A_{1 g}$ mode depicted in Figure $8.12 \mathrm{c}$ is a rare feature and only seen at hill sites locally heated with a $632.8 \mathrm{~nm}$ laser at full power. The unique topology and thermal transport constrictions in our $\mathrm{CuO}$ thin films allows us to probe both uniform and non-uniform laser heating. Similarly, when both hill and valley regions are sampled using a $514.5 \mathrm{~nm}$ laser at full power, we see evidence of two other changes to $\mathrm{CuO}$ : melting and a phase transition to $\mathrm{Cu}_{2} \mathrm{O}$.

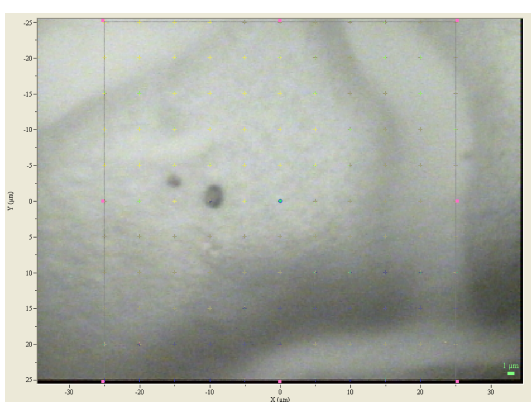

(a) Before acquisition

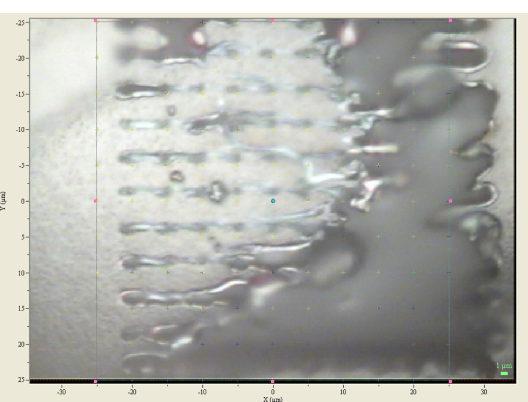

(b) After acquisition

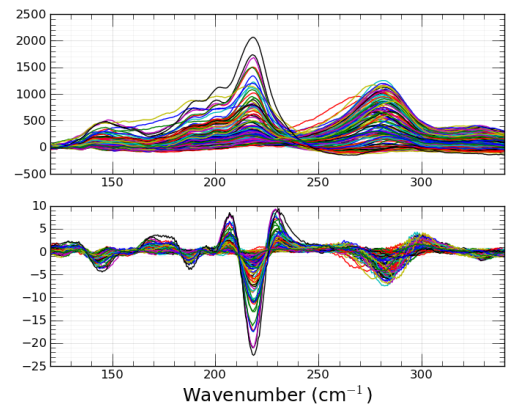

(c) Raman spectra

Figure 8.13: Full power spectra from three morphologies of $\mathrm{CuO}$.

Figure 8.13 shows optical images of the $\mathrm{CuO}$ sample before (Figure 8.13a) and after (Figure $8.13 \mathrm{~b}$ spatial mapping with a full power $514.5 \mathrm{~nm}$ laser. The resultant spectra are shown in Figure 8.13c. The image of the film after acquistion shows a laser trail both from the acquistion spot and from the laser moving across the sample while rastering to the next spot. The right hand part of the film also shows a complete change in contrast after acquisition and resembles each of the smaller circles on the left hand side suggesting that this entire area has been effectively decimated by the laser. From a visual inspection of the topology, this region appears to be a valley. In the previous section, we were able to find some evidence indicating that cooling by conduction is more likely at valleys than hills due to the reduced thermal cou- 
pling to the substrate and temperature-induced suppression of thermal conductivity. Hence, at hill sites, the heating is only local. At a valley site, the stronger connection to the substrate thermal sink and better thermal conductivity allow for thermal conduction. This spread of heat from the locally heated laser spot can affect a larger scale change as seen in Figure 8.13b.

As a final note, the laser trails in the optical image of the sample after acquisition show that the probed areas have a different contrast to the original $\mathrm{CuO}$. This either points to a change in the contrast from $\mathrm{Cu}_{2} \mathrm{O}$ - whose optical penetration depth is 10 times lower than $\mathrm{CuO}$ - or, that there is melting of the sample in addition to the phase transition.

\subsection{Conclusions}

We have shown that the temperature dependence of the both the peak position and linewidth of the $A_{1 g}$ Raman mode in $\mathrm{CuO}$ is due to a three phonon decay process. Here the Raman optical phonon decays into two daughter acoustic phonons of equal and opposite momenta. This temperature dependence due to global heating of the sample is also found to hold when the sample is locally heated by the laser.

The topology of the $\mathrm{CuO}$ thin film is one of undulating hills and valleys. The hill regions are where the $\mathrm{CuO}$ thin film has buckled and detached from the sapphire substrate in an effort to release some of the stress during the fabrication process. The stress arises from the two-fold volumetric expansion to the $\mathrm{CuO}$ structure. The nature of the evaporated film prohibits the expansion in the lateral directions so stress is released normal to the film. Since the hill regions are thermally isolated from the sapphire heat sink, they heat up considerably under the laser. At the same laser power and focus, the hills have an average temperature of $1000 \mathrm{~K}$ while the valley regions are around $400 \mathrm{~K}$. Thus, we can probe the changes to the Raman lineshape from $300 \mathrm{~K}$ to $1000 \mathrm{~K}$ using local heating. The results show that the linear correlation between $\omega_{\text {peak }}$ and $\Gamma$ found from the temperature dependence study holds to $1000 \mathrm{~K}$. From this we can infer (i) that there are no temperature-induced stress effects during local heating and (ii) that laser heating of the $\mathrm{CuO}$ thin film is mainly a homogeneous heating effect. This equivalence between local and global heating means that any temperature effects seen in nanostructures can be modelled using our the calibrations established in the temperature dependence study. The following chapter expands further on this idea for modelling the inhomogeneous temperature effects found in $\mathrm{CuO}$ nanowire forests using the calibrations found from homogeneous heating of the entire sample.

While local heating can be largely described with a homogeneous heating model, there are certain situations where a small degree of temperature inhomogeneity must be taken into account to describe the observed Raman lineshape. This inhomogeneity is seen in the full power 
spectrum taken within $3 \mu \mathrm{m}$ of the focal plane at a hill site. Under these conditions, the $A_{1 g}$ mode shows a bumpy asymmetry in low wavenmumber tail of the peak. This jutting shoulder occurs due to the inhomogeneous temperature distribution induced by the gaussian laser. The resultant lineshape is most likely due to a linear combination of Raman signal from regions at different temperatures.

The high optical absorption of $\mathrm{CuO}$ under a red laser with a wavelength of $632.8 \mathrm{~nm}$ can induce temperatures as high as $1000 \mathrm{~K}$. When the laser wavelength is decreased to $514.5 \mathrm{~nm}$, the degree of local heating is high enough to induce a phase transformation to $\mathrm{Cu}_{2} \mathrm{O}$. Under an atmospheric partial pressure of $\mathrm{O}_{2}$, this corresponds to temperatures around $1300 \mathrm{~K}$ in the sample. 


\section{Chapter 9}

\section{Raman spectroscopy of $\mathrm{CuO}$ nanowire forests}

\subsection{Introduction}

In Chapter 2.1.1, we saw that increasing phonon-phonon interactions at high temperatures leads to a symmetric broadening of the Raman lineshape. Under certain conditions, local heating by a gaussian laser beam can also lead to symmetric broadening. A homogeneous sample temperature can be established at the laser spot if there is adequate thermal contact with a heat sink i.e. the underlying substrate or the surrounding medium. In the $\mathrm{CuO}$ film, a symmetrically broadened $A_{1 g}$ peak was seen from regions where the film is physically connected to the substrate (valley regions; Figure 9.1a). Regions where the sample is physically disconnected from the heat sink (hill regions) showed broadening that could not be adequately described by a symmetric lineshape (Chapter 8.3.3). In the film, poor heat transport led to a spatially inhomogeneous temperature under the laser spot.

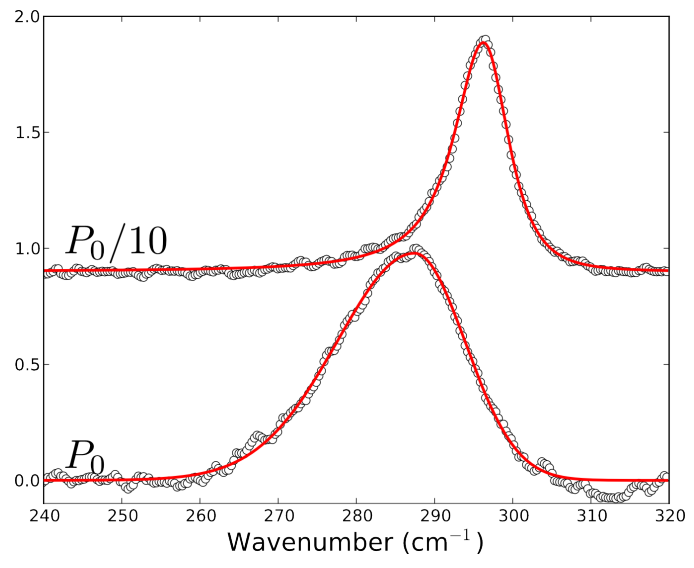

(a) Thin film

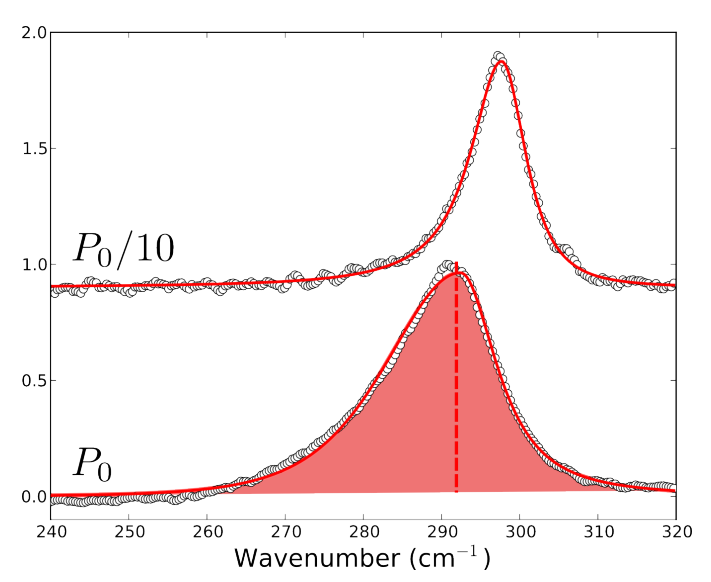

(b) Nanowire forest

Figure 9.1: Power dependence spectra for a thin film (a) and a nanowire forest (b).

A power dependent lineshape asymmetry - similar to that of silicon [191] - is also seen in 
$\mathrm{CuO}$ nanowire forests (Figure 9.1b). However, asymmetric broadening of Raman modes from nanostructures can have other physical origins such as phonon coupling with carrier continuum [162], phonon confinement [152] or a combination of phonon confinement and laser heating both homogeneous [166] and inhomogeneous [153]. In Chapter 7.3, we argued that thermal effects alone are the dominant mechanism behind the asymmetric lineshape from a $\mathrm{CuO}$ nanowire forest (Figure 9.1b). The thermal effects primarily involve the induced temperatures within the sample. In this chapter, we demonstrate that lineshape asymmetry can be modelled using a semi-emperical equation derived from the temperature dependence of the $\mathrm{A}_{1 g}$ mode from 'bulk' $\mathrm{CuO}$ (Chapter 8). The model calculates the minimum and maximum temperatures of the induced temperature distribution. We show that the asymmetric lineshapes are dependent on regions of homogeneous and inhomogeneous (spatial temperature gradients) temperature.

Regions directly under the laser hotspot experienced the largest rise in temperature. If the thermal conduction route to the substrate is disconnected, the only option is for the sample to cool by lateral thermal conduction (outwards from the hotspot). However, lateral thermal conduction is not very efficient at establishing a uniform temperature under the laser spot. Since thermal conductivity is a decreasing function of temperature, cooling rates are lower for regions with higher temperatures. Thus, gaussian laser beams induce a spatially inhomogeneous temperature across the illuminated regions of the sample. Raptis et al. [191] were able to show that temperature gradients induced by a laser with a large intensity distribution result in an asymmetric Raman lineshape in bulk silicon.

\subsection{Data collection}

Spatial maps from a nanowire forest were obtained taken at five different powers: FP, D0.3, D0.6, D1 and D2. As mentioned earlier, these power levels are fractions of the full power: $5-6.7$ $\mathrm{mW}$ (Table 2.1). For ease of acquisition, a flake of the $\mathrm{CuO}$ nanowire forest (Figure $7.11 \mathrm{~d}$ ) was stuck onto a glass microscope slide with water. Without this attachment to a surface, the flakes were highly susceptible to draughts. Spatial maps were collected with a minimum step size of 5 $\mu \mathrm{m}$ using an acquisition time of 10s. Raman spectra were smoothed and background-corrected according to the method described in Chapter 8.2. Around 230 spectra were collected in total.

\subsection{Quantifying Asymmetry}

Before setting up a model describing the lineshape as a result of temperature gradients, it is instructive to develop an approach to describe the asymmetry of the lineshape. Quantitative measurement of asymmetry can be done in a number of ways as it involves taking the width ratio from the two sides of the peak. For example, Adu et al. [153] defined the asymmetry 
parameter (henceforth referred to as 'skew') using the values of $\omega$ at $10 \%$ of the peak intensity. The low and high wavenumber sides of the peak are given as $\omega_{L}$ and $\omega_{R}$. The skew was calculated using: $\omega_{\text {peak }}-\omega_{L} / \omega_{R}-\omega_{\text {peak }}$. This method is sensitive to small asymmetry and long tailed peaks as it uses the width of the lineshape at $10 \%$ intensity. While useful for datasets where the asymmetry is mainly seen as a long tail, poor background correction and noisy data can introduce considerable errors into the calculation. The latter point is particularly relevant for low intensity datasets.

$$
\begin{aligned}
& I(\omega)=\begin{array}{llll}
a & 1+\frac{\left(\omega-\omega_{\text {peak }}\right)^{2}}{b^{2}} & -m_{1} & \text { if } \omega<\omega_{\text {peak }} \\
a & 1+\frac{\left(\omega-\omega_{\text {peak }}\right)^{2}}{b^{2}} & -m_{2} & \text { if } \omega>\omega_{\text {peak }}
\end{array} \\
& \Gamma=2 b \quad 2^{\frac{1}{m}}-1^{0.5}
\end{aligned}
$$

Fitting the datasets to an appropriate asymmetric lineshape function rids the problem of noisy data and the function fitting allows for the calculation of skew from the fitted FWHM. From a series of trials, the split-Pearson VII function - commonly used to fit XRD peaks provided the best empirical fits to our datasets. This function is considerably more flexible than a split-Lorentzian for fitting peaks with unusual tails. The formula is given in Eq. 9.1 and shows that the 'split' aspect simply couples two symmetric Pearson VII functions at the peak maximum. Figure 9.2 shows an example fit of Eq. 9.1. The suitability of the splitPearson VII function is apparent from the close fit of the function to a dataset that resembles a Lorentzian on the high wavenumber end and a medium-tail function on the low wavenumber end. The linewidth for each half of the peak is calculated using Eq. 9.2. $\Gamma_{\text {right }}$ represents the high wavenumber side and $\Gamma_{\text {left }}$, the low wavenumber side. The skew is defined as $\Gamma_{\text {left }} / \Gamma_{\text {right }}$.

Figure 9.3a shows a waterfall plot of asymmetric lineshapes fit with the SPVII function. The function fits a range of different peak shapes extremely well. However, there are a small number of cases where the observed asymmetry is not smooth and shows a 'double hump' feature e.g. Figure 9.3b. This feature can be related to the morphology of the nanowire forest.

As seen in Figure 7.19, the nanowires have lengths within a few microns and typically around $2 \mu \mathrm{m}$. These wires are well adhered to the oxide underlayer which has a thickness around $1 \mu \mathrm{m}$. Since the confocal depth of the Raman microscope is $6 \mu \mathrm{m}, 50 \%$ of the Raman signal could come $3 \mu \mathrm{m}$ away from the focus. In cases where the nanowires have a height less than 1-2 $\mu \mathrm{m}$ or regions where the wire density is sparse, signal from the polycrystalline oxide underlayer can be picked up. Spectra from such regions have a double hump as in Figure 9.3b. The main peak is from the nanowires while the broad shoulder comes from the oxide layer. All spectra with 


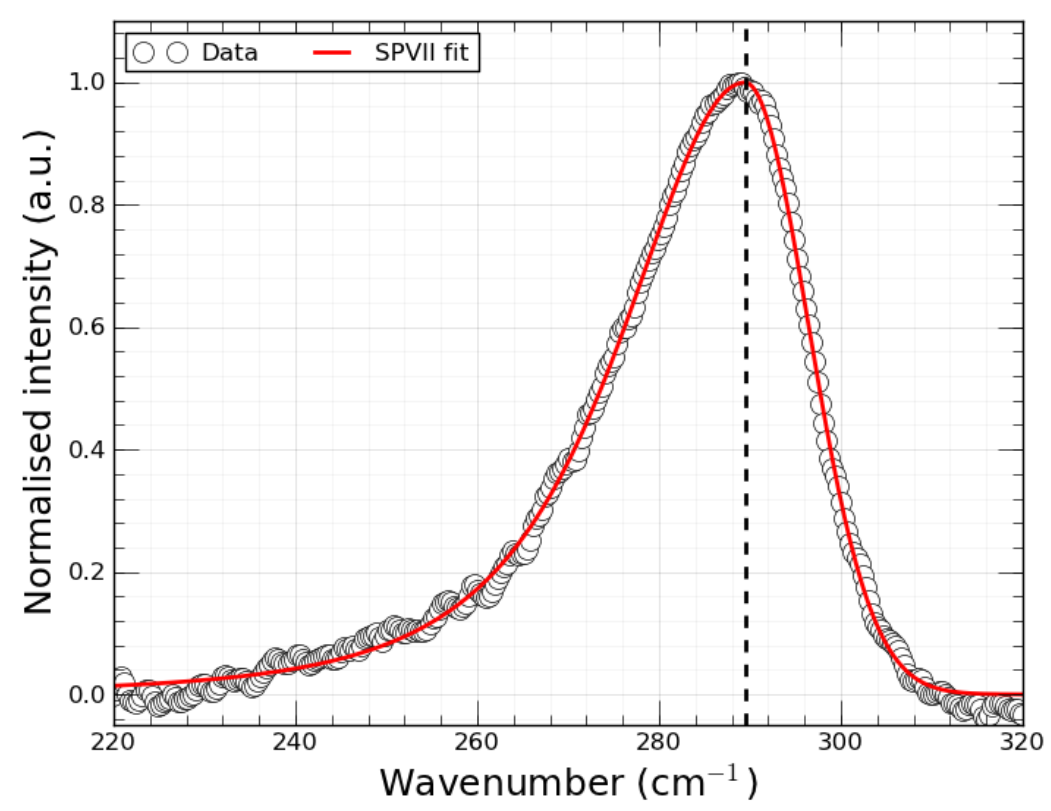

Figure 9.2: Asymmetric fitting using the split-Pearson VII function. The black dashed line depicts the peak center.

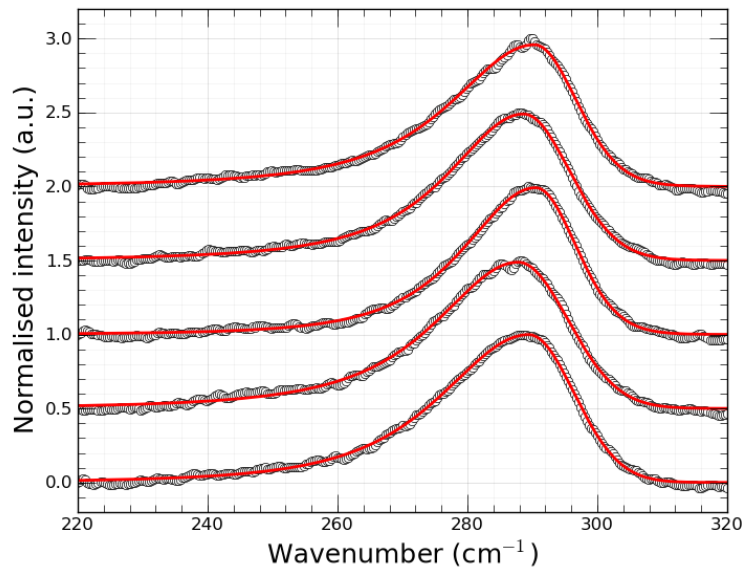

(a) Waterfall plot

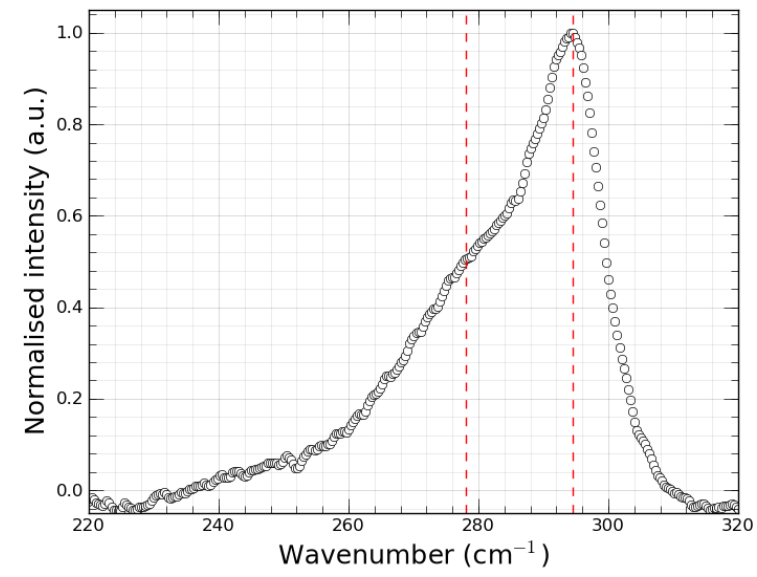

(b) Maximum skew

Figure 9.3: Spectral shape variation from different parts of the $\mathrm{CuO}$ nanowire forest. Smooth tailed spectra (a) vs. spectra with a 'double hump' feature (b).

this 'double hump' signature have a skew greater than 4.0, and are filtered from the analysis; the skew does not solely come from nanowires at different temperatures.

\subsubsection{Effective temperatures and asymmetry}

Eq. 9.1 was fit to each spectrum obtained from the spatial maps and the values of the fitted linewidths, peak positions, intensities and integrated intensities were extracted for further analysis. Since the asymmetric lineshape can be described with two linewidths, both the split- 
Pearson VII linewidths $-\Gamma_{\text {right }}$ and $\Gamma_{\text {left }}$ - are plotted against the peak position in Figure 9.4. The data from the temperature dependence study is also plotted for comparison.

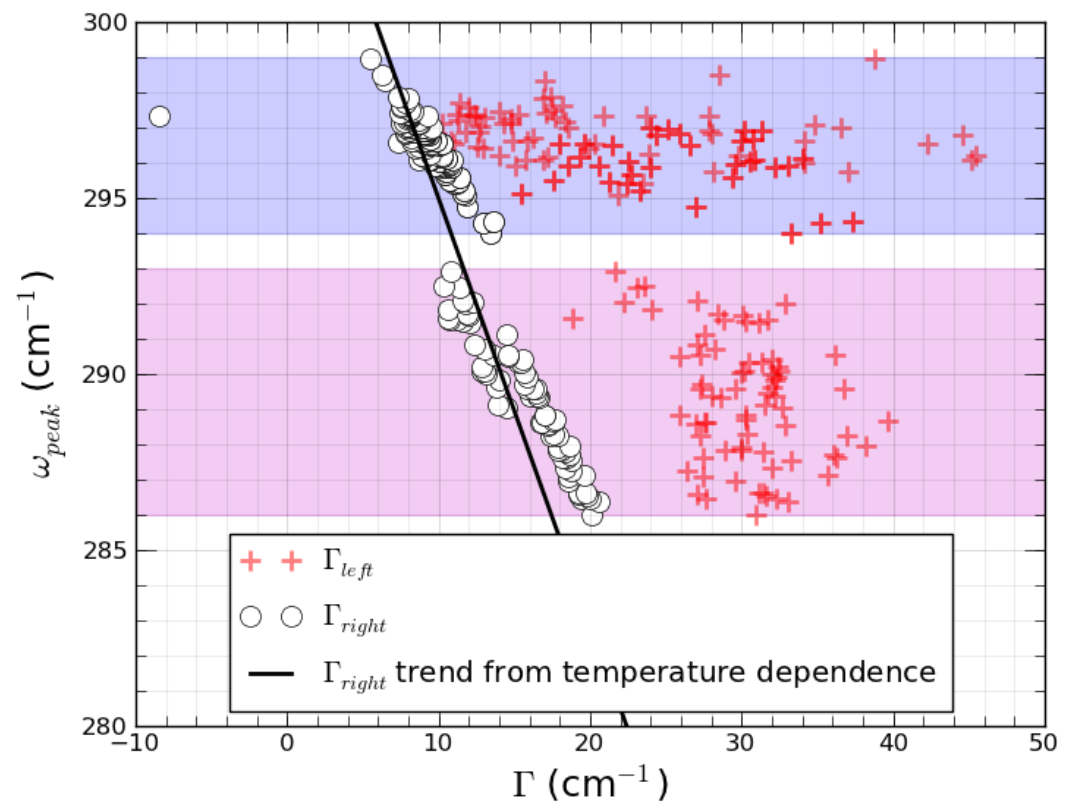

Figure 9.4: Plot of $\Gamma$ vs. $\omega_{\text {peak }}$ for the two sides of the fitted lineshapes..

Figure 9.4 highlights two significant features. The first is the linear trend between $\Gamma_{\text {right }}$ and $\omega_{\text {peak }}$ which follows the same relationship as the temperature dependence data observed in Chapter 8.2.2. This trend indicates that both $\omega_{\text {peak }}$ and $\Gamma_{\text {right }}$ are somehow related to a region at ambient or uniform temperature. The second is the sectioning of $\Gamma_{\text {left }}$ into two regions. One region (highlighted in blue) demonstrates a high variability in lineshape asymmetry and the other shows the asymmetry clustered about an average value (highlighted in red). The two regions appear to be separated at $\Gamma_{\text {right }}=10 \mathrm{~cm}^{-1}$.

Consequences from the different behaviours of $\Gamma_{\text {left }}$ for low and high values of $\Gamma_{\text {right }}$ are also observed in the power dependence of the skew. Figure 9.5a shows the skew for the same datasets plotted against $\Gamma_{\text {right }}$. The peak in the skew at $\Gamma_{\text {right }}=10 \mathrm{~cm}^{-1}$ corresponds to the gap in the data in Figure 9.4. Above this threshold, the skew decreases with increasing $\Gamma_{\text {right }}$. The opposite trend is seen for values of $\Gamma_{\text {right }}$ below this threshold. The colour-coding of the power-dependence data shows that the maximum skew is observed for laser powers of D0.3 and D0.6. The skew shows a decreasing trend for data collected at full power. Figure 9.5b shows the skew as a function of the peak position. For all laser powers except full power, the peak maximum of the $A_{1 g}$ mode is around $297 \mathrm{~cm}^{-1}$ but the skew can be as large as 4.0. This shows that despite large asymmetries in the peak shape, the centre of the peak stays in one position, until the power is increased to maximum. For spectra collected at full power, the peak position is red-shifted and the asymmetry is reduced. 


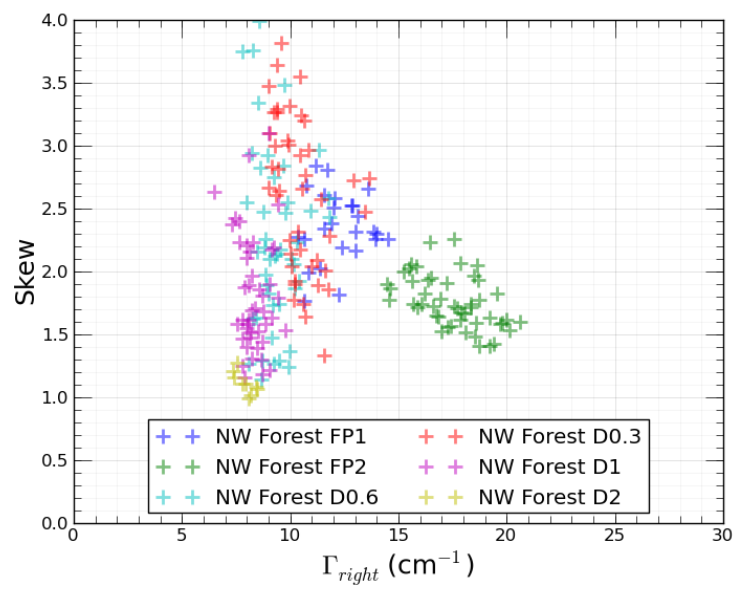

(a) Nanowire forest

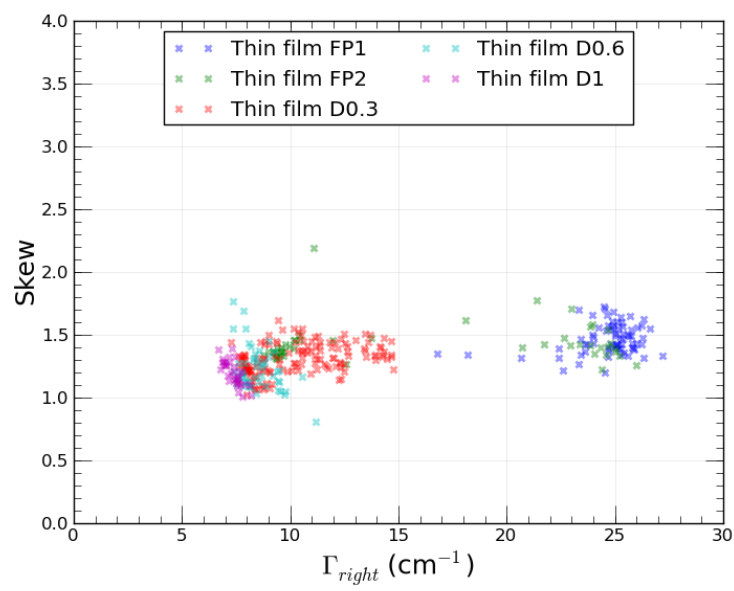

(c) Thin film

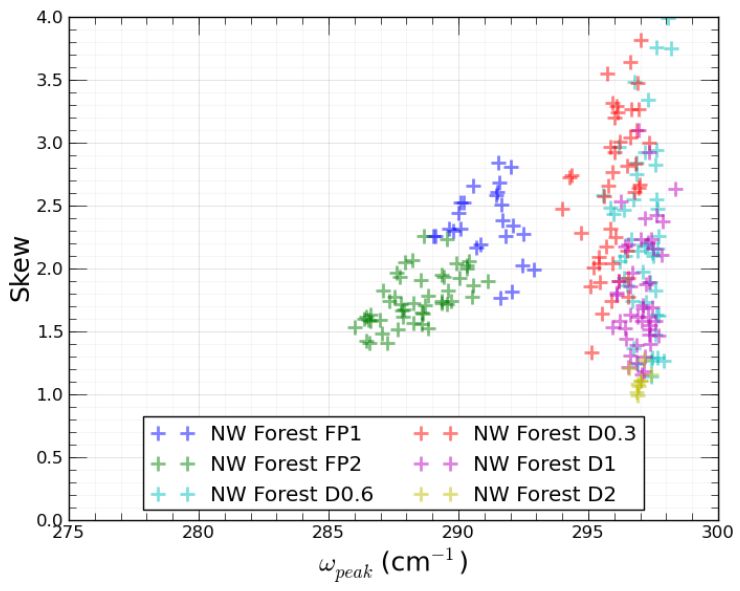

(b) Nanowire forest

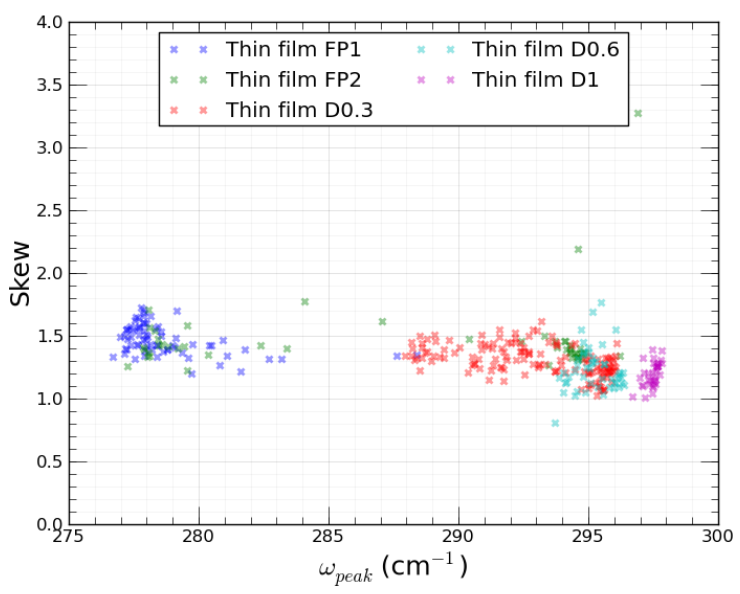

(d) Thin film

Figure 9.5: Lineshape asymmetry vs. $\Gamma_{\text {right }}$ (a) and $\omega_{\text {peak }}$ (b) at different powers for the nanowire forest. Lineshape asymmetry vs. $\Gamma_{\text {right }}(\mathrm{c})$ and $\omega_{\text {peak }}(\mathrm{d})$ for the thin film.

The same analysis was performed on the thin film data for comparison. The $\mathrm{A}_{1 g}$ mode was fit with a split-Pearson VII function instead of the pseudo-Voigt lineshape. Figure 9.5c shows a monotonously increasing trend of skew with $\Gamma_{\text {right }}$, indicating larger temperature gradients with increasing laser power. Similarly, there is a continuous decrease in $\omega_{\text {peak }}$ as skew gets larger (Figure 9.5d). The quadrant of plots in Figure 9.5 have the same axes limits. The difference between the variation and values of skew as a function of power is considerable between the thin film and the nanowire forest.

The clear power dependence in the skew for the nanowire forest indicates that the phenomenon is thermal in origin. As discussed in Chapter 7.3.2, inhomogeneous temperatures in the sample can lead to asymmetric Raman peaks. Instead of arriving from a region at a uniform temperature, Raman-scattered photons arrive from regions from across the temperature distribution. The larger the temperature distribution, the greater the asymmetry. However, de- 
viation from the trend of increasing skew with laser power at maximum power (in the nanowire forest) belies a physical change in the sample. A decrease in skew suggests a decrease in the spatial temperature distribution. The gaussian shape of the temperature distribution is 'flatter' due to a larger uniform temperature (red curve in Figure 9.6) though the skew parameter does not indicate if the maximum temperature also decreases. This flattening of the induced temperature gradients despite an increase in laser power suggests one of two possibilities: (i) destruction of the hottest regions of the sample by melting or phase change or, (ii) change in cooling mechanism.

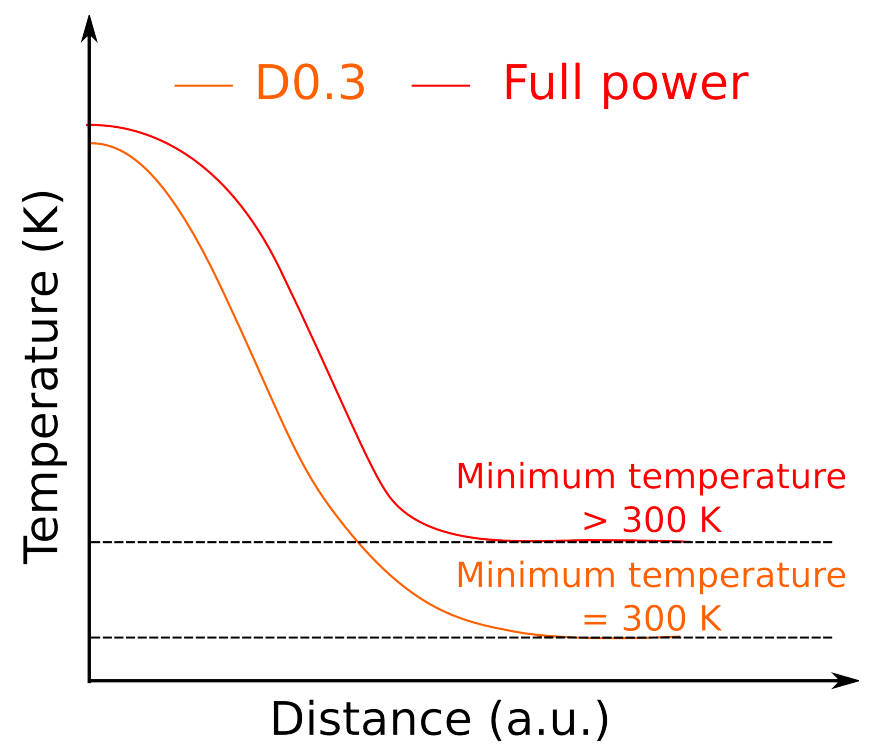

Figure 9.6: Hypothetical spatial temperature distribution at D0.3 vs. full power based on results of skew vs. $\Gamma_{\text {right }}$ seen in Figure $9.5 \mathrm{a}$.

$\mathrm{CuO}$ nanowires are susceptible to melting/decompostion under laser irradiation [192] though the authors' of this study did not point out any changes to the Raman lineshape during irradiation. We set up a simple experiment to correlate spectral features with post-acquistion SEM to check if the He-Ne laser was physically altering the nanowires. Instead of oxidising an entire copper button in the furnace, tracks were patterned on the surface with photolithography. The tracks of length $200 \mu \mathrm{m}$ and width $2 \mu \mathrm{m}$ exposed the copper surface. The remaining surface was covered with $10 \mathrm{~nm}$ of evaporated chromium. Nanowires were grown on the patterned substrate as described in Chapter 7.4. Following the growth, the entire length of the track was exposed to a length-wise linear mapping acquisition at full power. Linear maps were performed on several of the patterned tracks. Subsequent inspection of the substrate under the SEM did not reveal any features that could be specifically associated with laser-induced melting or decomposition (Appendix 1). Nanowires in the irradiated sample did not show any features that could be directly related to a laser beam moving down the center of the sample. Fused nanowires could be considered a consequence of local melting but this feature is also seen in control samples (Appendix 1). Spectra collected from the tracks showed only the CuO peaks.

The null result from this experiment agrees with the observations in Chapter 8.3.3. The 
$\mathrm{CuO}$ thin film only changed when exposed to a $514.5 \mathrm{~nm}$ laser (same laser used in the study of Yu et al. [192]) and these changes had both optical and spectral components. The regions of the film that had a visible alteration in morphology after acquisition also contained the Raman peaks of $\mathrm{Cu}_{2} \mathrm{O}$ in addition to $\mathrm{CuO}$ peaks. Thus, we suggest that sample melting/decomposition could be ruled out as the mechanism behind the decreasing temperature gradients observed in Figure 9.5a.

We suggest that the second option of a change in cooling mechanism above a critical temperature is more likely. Vertically oriented nanowire forests with a large surface area resemble cooling fins. Given the suppression of thermal conductivity due to the nanometer dimensions in relatively poor thermal conductor $\left(\kappa_{C u O} \sim 20 \mathrm{Wm}^{-1} \mathrm{~K}^{-1}\right.$ at $\left.38^{\circ} \mathrm{C}[193]\right)$, convection is the dominant cooling mechanism. The regions under the hotspot only continue to increase in temperature with increasing laser power until a critical point. Above this threshold, the reduced temperature gradients could be attributed to a more powerful cooling mechanism than convection: radiation. This suggestion can be tested by fitting the Raman lineshapes based on the induced temperature distribution. In the following section, two approaches to fitting are discussed.

\subsection{Modelling lineshape asymmetry}

This section details a semi-empirical model that fits the observed Raman lineshape based on laser-induced temperature distributions in the sample. We demonstrate that regions of both inhomogeneous and homogeneous temperature contribute to the Raman lineshape. The importance of the region at homogeneous temperature carries from the earlier discussion of the linear trend between $\Gamma_{\text {right }}$ and $\omega_{\text {peak }}$ (Section 9.3.1). We find that the critical threshold, above which the skew was seen to decrease (Figure 9.5), is due to a limiting temperature: between 1500 and $2000 \mathrm{~K}$. We suggest that this temperature denotes the onset of radiative cooling. We also suggest a possible connection between the morphology and the increasing ambient temperature seen in the full power spectra seen in Figure 9.5 .

\subsubsection{Model I: Inhomogeneous temperature distribution}

Figure 9.7a shows a simple planar model of inhomogeneous heating. The vertical nanowires see only a small portion of the laser beam. If the nanowires are sufficiently narrow, the laser intensity on a given nanowire can be taken as locally homogeneous. The local temperature is directly proportional to the laser intensity if the light is absorbed into a very small layer of the material and the thermal conductivity low enough to prevent fast heat dissipation. Regions at the center of the laser spot will heat up considerably more than the regions at the outside giving rise to spatial temperature distribution within the nanowire forest characterised by two param- 


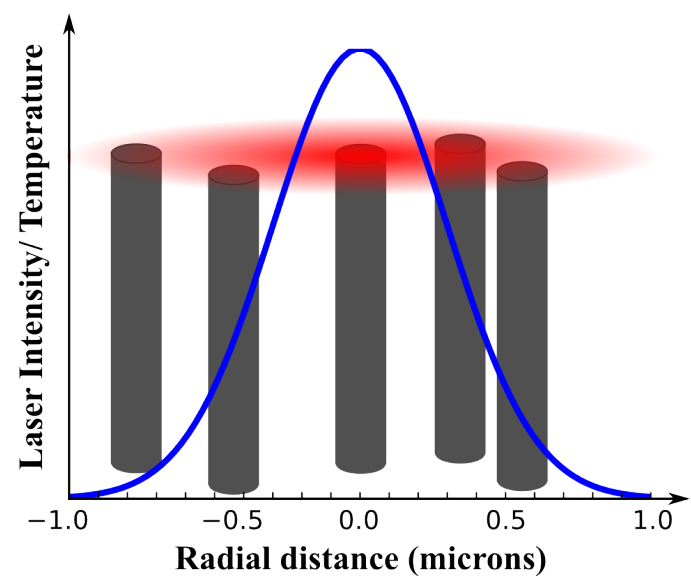

(a) Gaussian beam

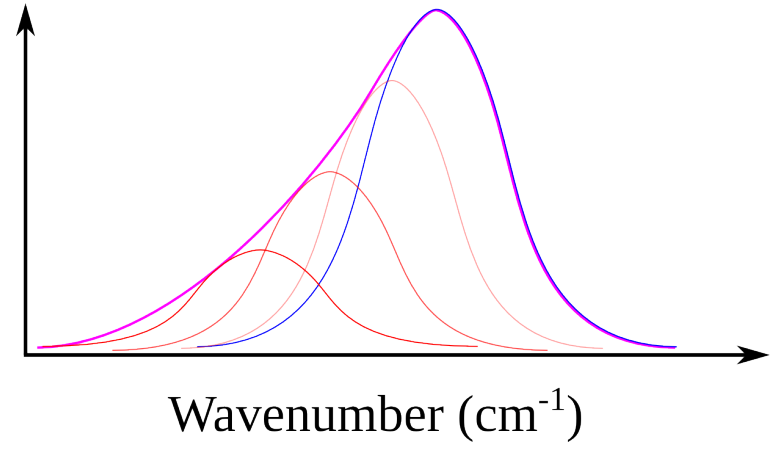

(b) Linear combination of signal

Figure 9.7: (a) Idealised picture of the effect of a gaussian beam on a perfectly vertical nanowire forest containing nanowires with a narrow diameter distribution. (b) Observed Raman signal decomposed into individual lorentzians from regions of different temperature.

eters: $T_{a}$ and $T_{\max }$; the lowest and highest induced temperatures. the temperature dependence of Raman signal determines the contribution of a region at some temperature $T_{i}$ to the observed Raman signal. As discussed in the previous chapter, the Raman signal of $\mathrm{CuO}$ decreases as a function of temperature. Thus, the observed Raman signal can be assumed to be a linear combination of the Raman signal from each of these nanowires which have some temperature $T_{i}$. Though the region of $T_{\max }$ receives the highest flux of laser photons, the Raman signal from this area is low compared to a region close to $T_{a}$ since the induced temperature is much higher. This arrives directly from the temperature dependence of the Raman intensity seen in Chapter 8.2.2. Since the $\mathrm{A}_{1 g}$ mode broadens and red-shifts at higher temperatures, the linear combination of signal leads to a Raman lineshape with an asymmetry on the low wavenumber side (Figure 9.7b).

The planar model provides a simplistic description of how an inhomogeneous temperature distribution results in an asymmetric Raman lineshape. This model would suit the observations of Raptis et al. on bulk silicon [191] but not a nanowire forest that is exposed to the full laser intensity cone (the gaussian beam also has a $z$ dependence on intensity). The intensity distribution of the beam is much larger in the propagation direction compared to the lateral direction (Chapter 2.2.2). If the nanowires have an average length of $1-2 \mu \mathrm{m}$, the entire wire is exposed to a laser intensity that is determined only by the lateral intensity distribution and is unchanged over the length of the nanowire. Since wire diameter are typically $100 \mathrm{~nm}$ or less [137, the full wire is heated to some temperature $T_{i}$ that is proportional to the laser intensity. Thus, we can assume that the entire wire is hot and the two thermal sinks are polycyrstalline $\mathrm{CuO}$ layer at the base of the nanowire and the air surrounding the wire. The surface area of the surrounding air is far larger than the small contact point that connects the wire to the $\mathrm{CuO}$ layer and the dominant cooling mechanism in the hot nanowires is likely to be convection. 


\section{Derivation of inhomogeneous temperature model}

The observed Raman signal from a region illuminated by a gaussian beam can be given as:

$$
\text { Signal }={ }_{r=0}^{\mathbb{Z}_{\infty}} R\left(r, \omega_{\text {peak }}, \Gamma, I\right) I(r) r d r
$$

If the temperature induced by the laser beam is uniform across the illuminated area, the entire integral in Eq. 9.3 can be simplified by $R\left(\omega_{\text {peak }}, \gamma, I\right)$ : the unique Raman lineshape described by a peak position $\left(\omega_{\text {peak }}\right)$, linewidth $(\Gamma)$ and intensity $(I)$. If the induced temperature is a function of the laser intensity, Eq. 9.3 holds as an integral; the observed Raman lineshape is a linear combination of Raman signal from regions at different temperatures. The expression in Eq. 9.3 can be simplified to an integrable function in a few steps that not only leads to a functional form that can be used for fitting but also provides some physical insight. Changing the integration parameter from $r$ to $T$ is the major step towards a simpler functional form. This transformation is required since the spatial temperature distribution is unknown, other than its gaussian-like shape (Chapter 2.1.2). Eqs 9.4 to 9.12 detail this transformation.

The term $I(r)$ can be simplified as $I_{0} \exp ^{-\alpha r^{2}}$ for a gaussian beam. As temperature is solely a function of $r$, the induced temperature in the sample can be simplified as: $A I_{0} \exp ^{-\alpha r^{2}}$. The constant, $A$, is the absorbance of the sample. The heated nanowire forest can be assumed to cool by convection cooling to ambient temperature $\left(T_{a}\right)$. The radiative cooling mechanism can be assumeed unimportant in a system that resembles a cooling fin; $\mathrm{CuO}$ nanowires have aspect ratios greater than 10. Hence, the two sides of the heat equation ('heat in' and 'heat out') in the steady state can be written as:

$$
Q_{\text {in }}=Q_{\text {out }} A I_{0} \exp ^{-\alpha r^{2}}=\gamma\left(T-T_{a}\right)
$$

The constants in Eq. 9.4 can be lumped together into the term, $L$, and the gaussian term can be shortened as $\beta(T)$ :

$$
\beta(T)=\exp ^{-\alpha r^{2}}=L T
$$

From this equation, $d r / d T$, can be written as:

$$
\frac{d r}{d T}=\frac{-L}{2 \alpha r \beta(T)}
$$

The transformation of co-ordinates in Eq. 9.3 from $r$ to $T$ is now possible with Eq. 9.7. 
All terms associated with $r$ are change to their equivalents in terms of $T$. The integration limits, $T_{a}$ and $T_{\max }$, represent the temperature at the extreme ends of the spatial temperature distribution. At the center of the spot, $r=0$, the temperature is given by $T_{\max }$. The flattened tail of the distribution, when $r \rightarrow \infty$, the temperature is $T_{a}$.

$$
\text { Signal }=\underbrace{\mathbb{Z}_{\max }}_{T_{a}} R\left(\omega_{\text {peak }}, \Gamma, I\right) I(T) r(T) \frac{d r}{d T} d T
$$

The term, $r(T)$, can obtained by integrating $d r / d T$ from Eq. 9.6. $I(T)$ can be expressed as $I_{0} \beta(T)$. Cancellations involving the $r(T)$ term and the term within $d r / d T$, and the $\beta(T)$ term in $d r / d T$ and $I(T)$ leave:

$$
\begin{aligned}
& \text { Signal }=-\underbrace{\mathbb{Z}_{\max }}_{T_{a}} R\left(\omega_{\text {peak }}, \Gamma, I\right) \frac{I_{0} L}{2 \alpha} d T \\
& \underline{Z} \max \\
& =-K \quad R\left(\omega_{\text {peak }}, \Gamma, I\right) d T \\
& T_{a} \\
& Z_{\max } \\
& =K \quad R\left(\omega_{\text {peak }}, \Gamma, I\right) d T \\
& T_{a} \\
& R\left(\omega_{\text {peak }}, \Gamma, I\right)=I_{\text {Raman }}(T) \times L(\omega, T) \\
& =\exp \left(-4.5 \times 10^{-3} T\right) \frac{1.0}{\left(\exp \left(h c \omega_{0} / k_{B} T\right)-1\right)}+1 \\
& \times \eta \frac{(\Gamma(T) / 2)^{2}}{[\omega-\omega(T)]^{2}+(\Gamma(T) / 2)^{2}}+(1-\eta) \exp \frac{(\omega-\omega(T))^{2}}{\Gamma(T)^{2} / 4 \ln (2)}
\end{aligned}
$$

The term, $R\left(\omega_{\text {peak }}, \Gamma, I\right)$ is comprised of the temperature dependent forms of the peak position, linewidth and intensity. The empirical relations of these parameters were identified in the previous chapter. Thus, $R\left(\omega_{\text {peak }}, \Gamma, I\right)$ can be written as shown in Eq. 9.12. The function, $L(\omega, T)$, describes the pseudo-Voigt lineshape of the $A_{1 g}$ peak as a function of wavenumber and temperature, while $I_{\text {Raman }}$, represents the $\mathrm{A}_{1 g}$ intensity as a function of temperature. The Raman intensity was attributed as either linear or exponental in Chapter 8.2.2 though the 
exponential dependence was favoured since it allowed the Raman signal to remain non-zero above $800 \mathrm{~K}$. As laser-induced temperatures can be larger than $800 \mathrm{~K}$ (Chapter 8.3.2), the exponential dependence identified in the previous chapter was directly substituted here as $I_{\text {Raman }}$.

It should be noted that the observed Raman lineshape, $R\left(\omega_{\text {peak }}, \Gamma, I\right)$, is in fact a composite since it is comprised of signal from several regions within the sample. The Raman signal from an individual region (at some temperature, $T_{i}$ ) is represnted by the pseudo-Voigt lineshape (Chapter 8.2). However, to avoid confusion by abruptly changing the terminology, the reader is advised to consider all the following instances of 'the observed Raman lineshape' to indicate the composite.

\section{Fitting procedure}

The final equation of the Raman signal arising from a sample with an inhomogenous temperature distribution is Eq. 9.10. The Raman data is fitted in two steps: the first step calculates the contribution for a given wavenumber, $\omega_{i}$ ( between 220 and $320 \mathrm{~cm}^{-1}$ ), from a region at some temperature, $T_{i}$ (between $T_{a}$ and $T_{\max }$ ). This calculation is encapsulated within a function which is optimised to give the observed Raman lineshape.

\section{Fitting results}

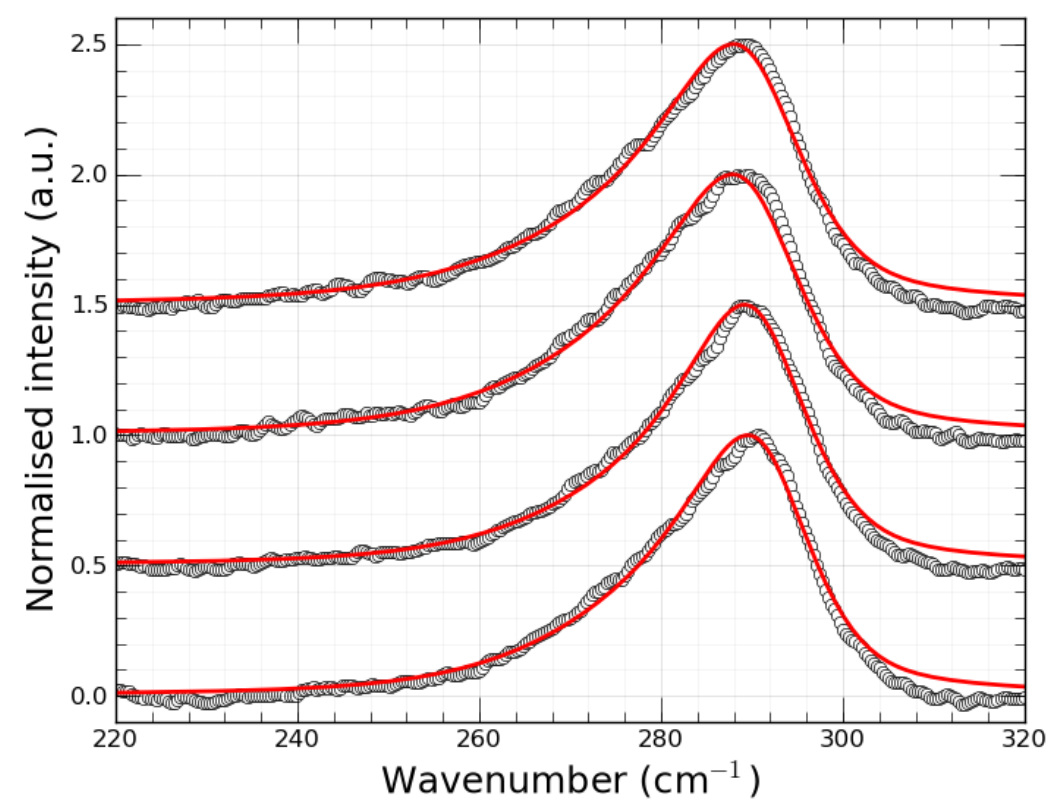

Figure 9.8: Randomly selected full power spectra from a spatial map fitted with Eq 9.10.

The $\mathrm{A}_{1 g}$ mode from spatial mapping measurements at five different power levels was fitted according to the description in Section 9.4.1. Figure 9.8 shows the fits to a few datasets col- 
lected at full power. Figure 9.9a shows the fitted values of $T_{\max }$ plotted against $T_{a}$. Figure 9.9b shows the mean and standard deviation of $T_{a}$ and $T_{\max }$ for each of the six datasets. These plots clearly show two regimes for $T_{\max }$ : one where $T_{\max }$ is steadily increasing and another where it appears to have saturated. The saturation temperature is around $1200 \mathrm{~K}$. The behaviour of $T_{a}$ in the first regime is quite unusual: $T_{a}$ decreases as $T_{\max }$ increases. This decrease merits some attention as $T_{a}$ not only deviates to unphyiscal temperatures but also deviates from $T\left(\Gamma_{\text {right }}\right)$.

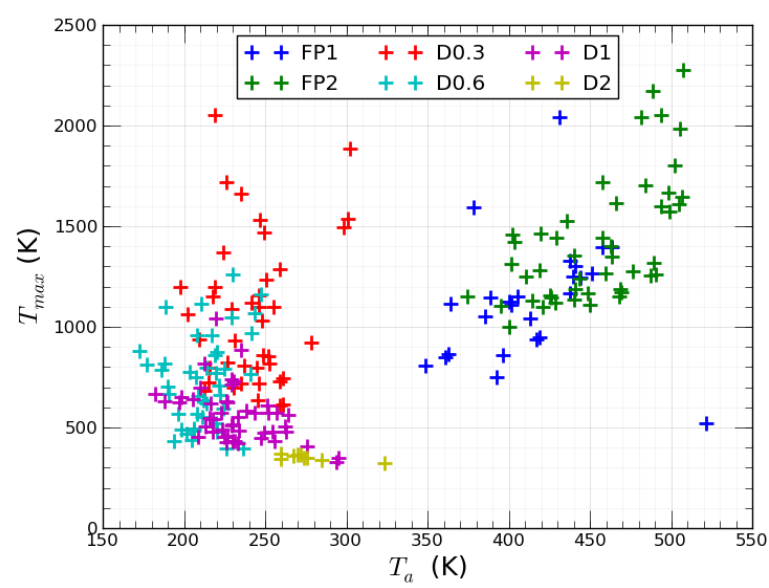

(a) $T_{a}$ vs. $T_{\max }$

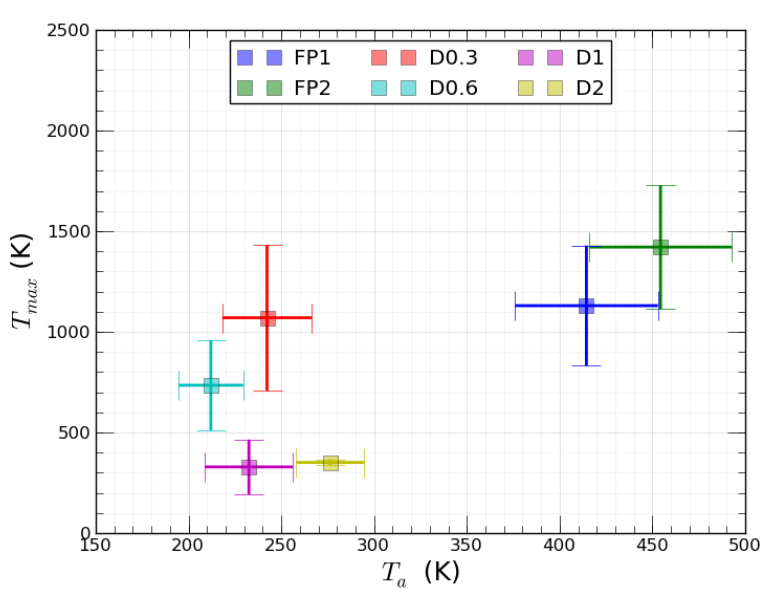

(b) $T_{a}$ vs. $T_{\max }$ statistics

Figure 9.9: Summary of fitting nanowire forest spectra. (a) All points from fitting. (b) Summary statistics by power level.

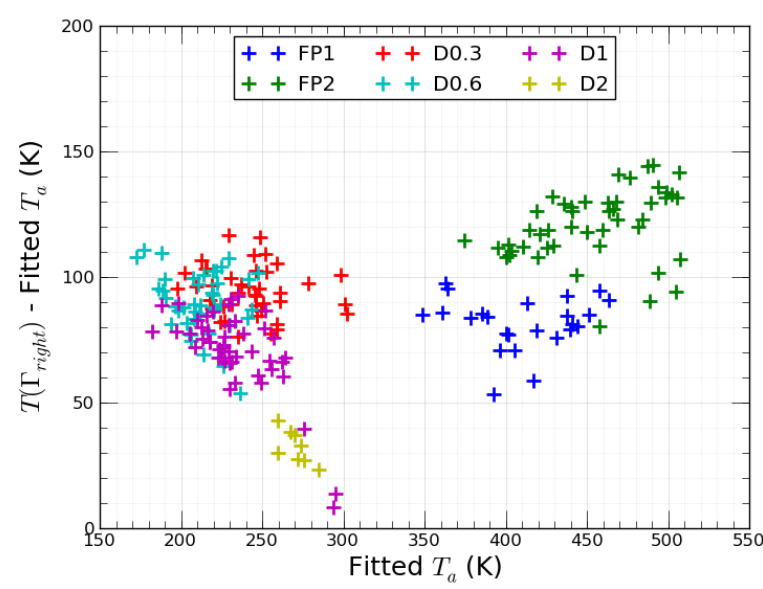

(a) $T\left(\Gamma_{\text {right }}\right)-T_{a}$

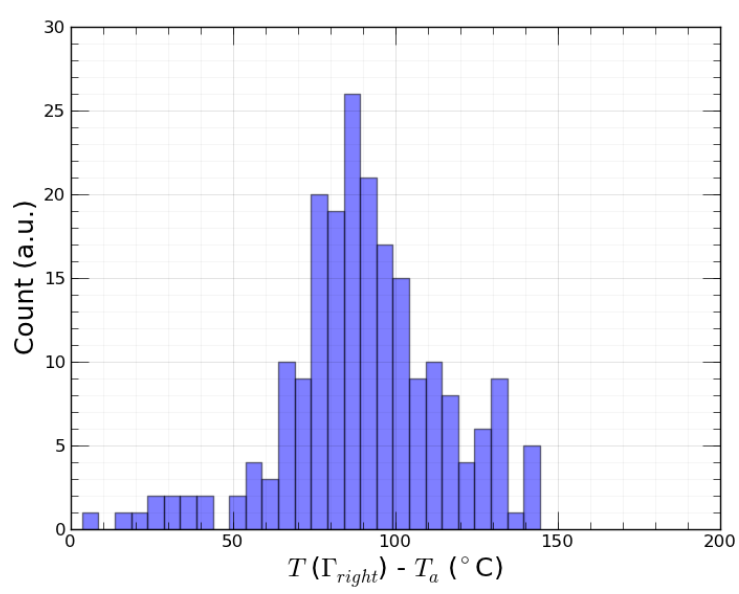

(b) $T\left(\Gamma_{\text {right }}\right)-T_{a}$ histogram

Figure 9.10: Difference between fitted $T_{a}$ and $T\left(\Gamma_{\text {right }}\right)$.

As per the brief discussion in Section 9.3.1, both $\Gamma_{\text {right }}$ and $\omega_{\text {peak }}$ were connected to a region of uniform temperature. For all powers except full power, this region had a temperature around $300 \mathrm{~K}$. During the conception of the inhomogeneous temperature model, the value of $T_{a}$ was expected to agree with $T\left(\Gamma_{\text {right }}\right)$. However, the fitted values of $T_{a}$ that this is not the case. 
Figure 9.10a shows the difference between $T_{a}$ and $T\left(\Gamma_{\text {right }}\right)$. Only the spectra obtained with a power level of D2 show a small deviation of around $30{ }^{\circ} \mathrm{C}$. The remaining datasets deviate by around $100{ }^{\circ} \mathrm{C}$ as shown in Figure $9.10 \mathrm{~b}$.

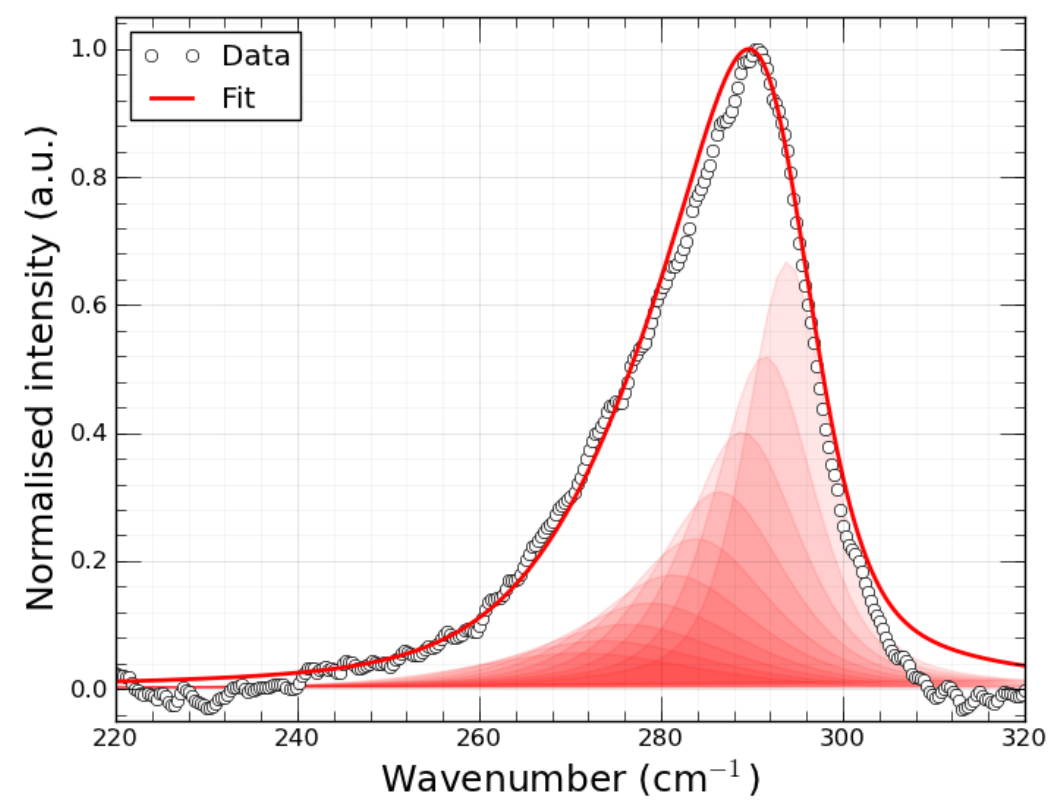

Figure 9.11: Selected components of ihomogeneous temperature distribution model fitted to an asymmetric spectrum obtained at full power. The component peaks have been scaled by a factor of 3 for clarity.

The source of this discrepancy can be traced to the model: the way the model fits the data requires the region of maximum signal at a higher wavenumber than the peak position. Figure 9.11 shows a selected number of the fitted components between $T_{a}$ and $T_{\max }$. The component belonging to the region with maximum signal, $T_{a}$, is seen to fit within $\Gamma_{\text {right }}$. Thus, $T_{a}$ is lower than $T\left(\Gamma_{\text {right }}\right)$. Similar visual analysis of other datasets show that this is a systemic feature.

The aspect of the inhomogeneous temperature model that results in the under-estimation of $T_{a}$ is quite simple: it completely ignores regions at homogeneous temperature. The Raman signal is entirely described by regions at different temperature: all with equal volume. $T_{a}$ needs to be included in the fitting as a region with uniform temperature that also contributes to the Raman lineshape. The poor fitting of $T_{a}$ also indicates that it does not fit into the equal volume picture used to simplify the description of the inhomogeneous temperature region. In the following section, we correct the inhomogeneous temperature model to include a region of homogeneous temperature with a different signal volume to the region under a temperature distribution. 


\subsubsection{Model II: Inhomogeneous + Homogeneous temperature dis- tribution}

$\mathbb{Z} \max$

$$
\begin{aligned}
& R\left(\omega_{\text {peak }}, \Gamma, I\right)=K \quad\left[I_{\text {Raman }}(T) \times L\left(\omega_{i}, T\right)\right]+\left[V_{T_{a}} I_{\text {Raman }}\left(T_{a}\right) \times L\left(\omega_{i}, T a\right)\right] d T \\
& T_{a}
\end{aligned}
$$

Modifying the inhomogeneous temperature model to include an independent component from a region at $T_{a}$ is relatively simple. Eq. 9.13 shows that the observed Raman lineshape is now a sum of contributions from an inhomogeneous region and a homogeneous region. The contribution from the homogeneous region is modulated by the parameter, $V_{T_{a}}$, to account for variable signal from this region. Ignoring this parameter results in an extremely poor fit. The final modification was constraining the value of $T_{a}$ as $T\left(\Gamma_{\text {right }}\right)$ in order to reduce the complexity and time of fitting. This modified model provides an excellent fit to the full range of powerdependent spectra. Figure 9.12a shows a sample fit to the data using Eq. 9.13 as $R\left(\omega_{\text {peak }}, \Gamma, I\right)$ in Eq. 9.10 for all five power levels.

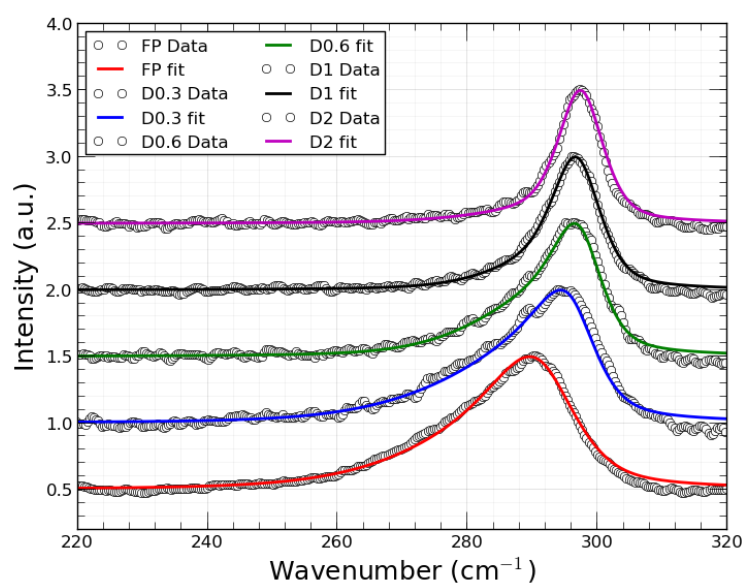

(a) Fits to Eq. 9.13

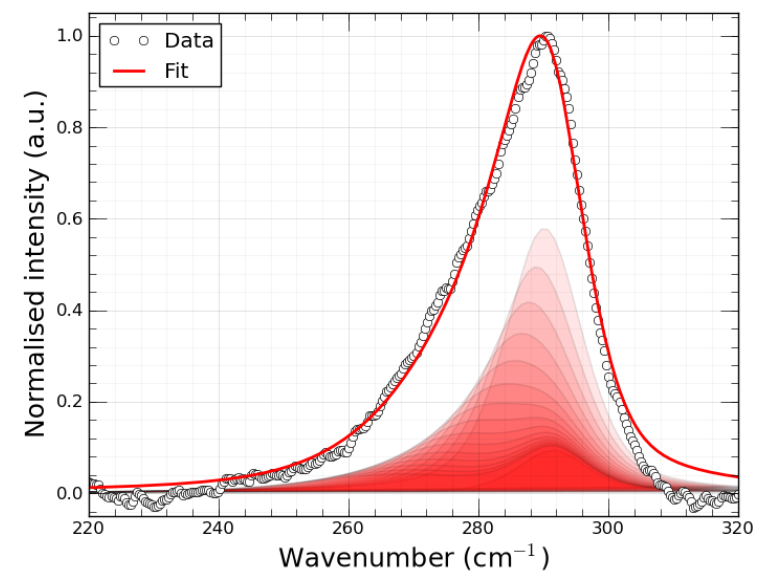

(b) Selected components

Figure 9.12: (a) Fits to Eq. 9.10 (with Eq. 9.13 as $\left.R\left(\omega_{\text {peak }}, \Gamma, I\right)\right)$ for a representative spectrum from each of the five power levels. (b) Selected components of inhomogeneous + homogeneous temperature model fitted to an asymmetric spectrum obtained at full power. The component peaks have been scaled for clarity.

Figure $9.12 \mathrm{~b}$ shows a sample spectrum, and a selection of the components between $T_{a}$ and $T_{\text {max }}$, fitted with the modified model in Eq. 9.13. Constraining the lower integral limit to $T\left(\Gamma_{\text {right }}\right)$, and accounting for a variable region at temperature $T_{a}$ results in an excellent fit to the data. The fitting results for all the datasets are summarised in Figure 9.13a and 9.13b. The previous observation of a limiting $T_{\max }$ is also seen here, albeight at a higher temperature: between 1500 and $2000 \mathrm{~K}$. The unusual feature in this set of fitting is the large $T_{\max }$ for data obtained with a very low laser power: D2 (1\% of maximum power). The spectra obtained at this power show virtually no asymmetry (Figure 9.5a) yet, the fitting indicates that $T_{\max }$ is 
around $1000 \mathrm{~K}$.

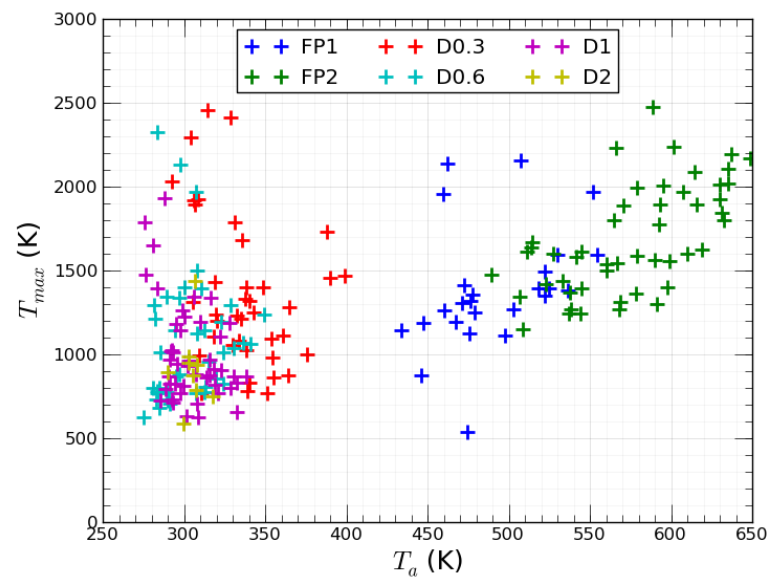

(a) $T_{a}$ vs. $T_{\max }$

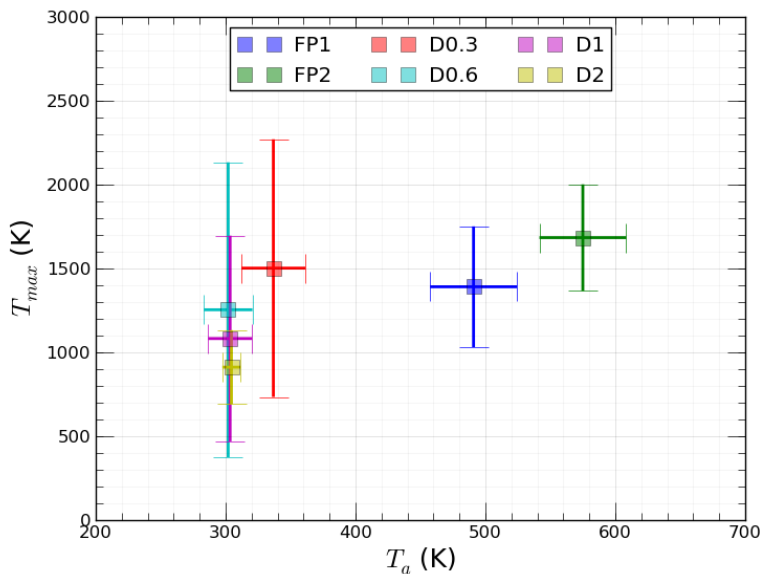

(b) $T_{a}$ vs. $T_{\max }$ statistics

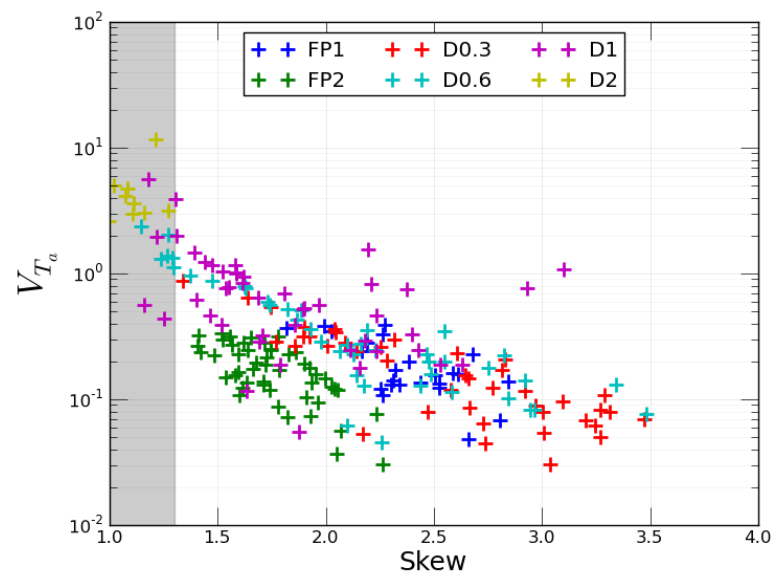

(c) $V_{T_{a}}$ vs. skew

Figure 9.13: Summary of fitting Model II to nanowire forest spectra. (a) All points from fitting (b) Summary statistics by power level. (c) Plot of $V_{T_{a}}$ vs. skew.

This unusual result can be reconciled when the parameter, $V_{T_{a}}$, is considered. Figure $9.13 \mathrm{c}$ shows a plot of $V_{T_{a}}$ against skew. The region of small skew, below 1.3, is highlighted in grey. The majority of the data in this low skew region - particularly the dataset at D2 - have large values of $V_{T_{a}}$ : well above 1.0. The value of the temperature-dependent Stokes intensity is around 1.0 at $300 \mathrm{~K}$ (Figure 8.5). Hence, a large $V_{T_{a}}$ indicates a signal several times greater than the signal from temperatures above $T_{a}$ - which would have values below 1.0.

Two significant trends seen from the fits to Model I are also found in the fits to Model II: (i) limiting value of $T_{\max }$ and (ii) the increase in $T_{a}$. Both these trends suggest that the flattened temperature distribution for nanowires under full power - which results in smaller skew compared to spectra obtained at lower power (Figure 9.5 - is mainly due to larger $T_{a}$. The physical reasons for each of the two trends however, are different. The saturated value of 
$T_{\max }$ suggests a change in the properties of the cooling mechanism while the increase in $T_{a}$ is not. Instead, it may be correlated with the variable coverage of $\mathrm{CuO}$ nanowires within the forest.

Both Model I and II rely on convection as the dominant cooling mechanism. According to this mechanism, heat out is proportional to heat in (providing the ambient temperature remains the same). However, the increase in the incoming heat from the laser does not translate to an increase in temperature. Instead, the temperature remains constant. This could be associated to destruction of nanowires with temperatures above this constant temperature. However, a visual examination of nanowire regions exposed to the laser beam found no obvious evidence of nanowire destruction by melting. And, the spectral signatures were always associated with $\mathrm{CuO}$, so decomposition to $\mathrm{Cu}_{2} \mathrm{O}$ was also ruled out. The only remaining explanation is a radical change in the 'heat out' component of the steady state heat equation. Heat transfer is a complicated process and our insight into these processes in $\mathrm{CuO}$ nanowire forests is indirect. Hence, we can only speculate that one radical change is the onset of radiative cooling at a critical temperature: the limiting value of $T_{\max }$.

$T_{a}$, on the other hand, seems to be unrelated to a fundamental change in sample properties. The collection cone of the objective used in this study can be calculated to be greater than the illuminated area of the laser spot (Chapter 2.2.5). At $r_{\infty}$, when $T \rightarrow T_{a}$, the temperature is expected to be around $300 \mathrm{~K}$. Yet, this ambient temperature increases to a large spread of values between 450 and $650 \mathrm{~K}$. There is no pattern in this spread. Instead, the underlying mechanism can be linked to physical characteristics of the experimental conditions rather than changes in the sample properties.

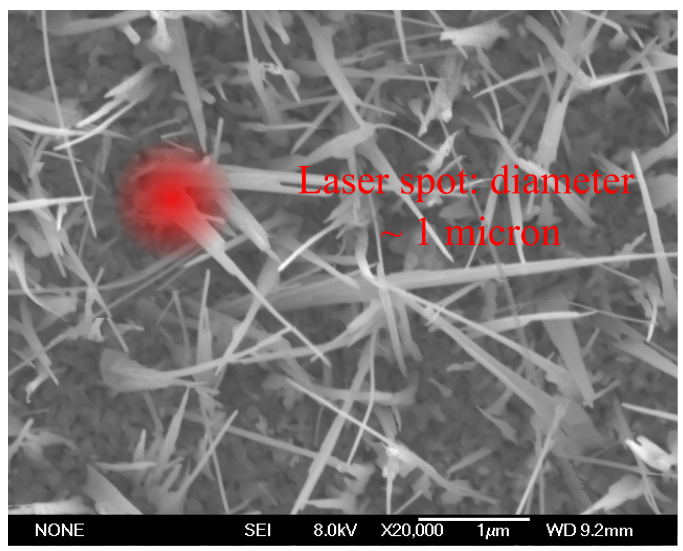

(a) Laser spot

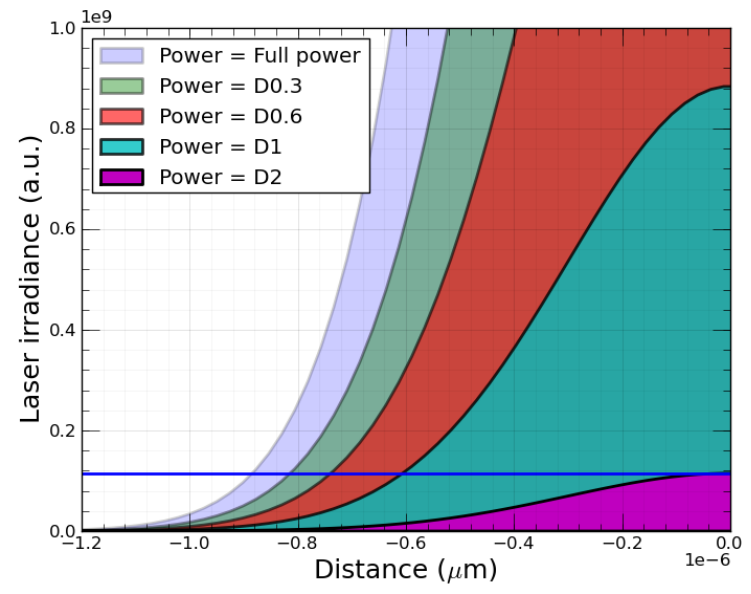

(b) Signal depth

Figure 9.14: (a) Size of the laser spot and (b) Laser irradiance at the different power levels. The horizontal blue line traces the maximum laser irradiance at D2.

In a nanowire forest with a high degree of spatial variation, the experimental conditions are strongly linked to the local coverage of nanowires. Figure 9.14a shows an SEM image with a 
superimposed red circle that represents the Raman laser spot with a diameter of $1 \mu \mathrm{m}$. The region at $T_{a}$ is a thin annulus. If $T_{a}$ is $300 \mathrm{~K}$, the inner edge of the annulus corresponds to a value of the laser power that does not induce any heating. For $\mathrm{CuO}$ nanowires, this value corresponds to $1 \%$ of maximum power (power level D2). The outer edge of the annulus corresponds to some critical power level, below which Raman signal is swallowed into the noise. As power levels increase, the area of this annulus gets smaller and smaller. Figure 9.14b shows half of the gaussian beam curve for the different power levels used in this study. The curves are normalised by the maximum value at full power. The threshold of the inner annulus, dictated by the maximum power at D2, is indicated by the horizontal blue line. The intersection of this line with the laser power curves shows that the inner annulus is pushed further away as laser power increases. If the critical threshold for the outer edge is a constant, the area of the annulus decreases with increasing power. This characteristic has a powerful implication when combined with the erratic coverage of $\mathrm{CuO}$ nanowires in the forest: the probability of finding nanowires in this annulus decreases along with the annulus area. Thus, the value of $T_{a}$ may no longer be $300 \mathrm{~K}$. We believe that this picture also supports the large variation in $T_{a}$ at full power.

The main source of uncertainty in this model, that results in a broad band of values for $T_{a}$ and $T_{\max }$, is due to the highly variable sample geometry. With a better sample - vertically oriented nanowires with a narrow diameter and length distribution - the characteristics of the sample can be used to deduce several useful parameters. Using spectra taken at several powers with different objectives, one could identify the spatial extent of homogeneous heating. Similarly, the region around the hotspot where radiative cooling takes over can also be assigned as a function of the nanowire diameter. Vertically oriented nanowires can be fabricated [142] but require some time spent optimising the fabrication process.

\subsection{Conclusions}

We have presented a novel set of Raman data from $\mathrm{CuO}$ nanowire forests that exhibit highly asymmetric lineshapes. The lineshapes can be fit with an empirical split-peak function to quantify widths for each half. The skew, ratio of these two widths, rises with increasing laser power until a critical power level is reached. In our system, this power is around 5-6.7 $\mathrm{mW}$. Above this level, the skew begins to decrease. This decrease corresponds to a flattened spatial temperature distribution (red curve in Figure 9.15). We have derived and fitted an appropriate model that fits the observed Raman lineshapes based on the temperature distributions induced in the sample. We found that two types of temperature regions are required to adequately fit the experimental data: a region of inhomogeneous temperatures and a region at uniform temperature. From the fits to this model, we found that the flattened temperature distributions at full power arise solely from a rise in the ambient temperature. The maximum induced temperature reaches a critical value, between 1200 and $1600 \mathrm{~K}$, at $50 \%$ of full power (D0.3, orange 


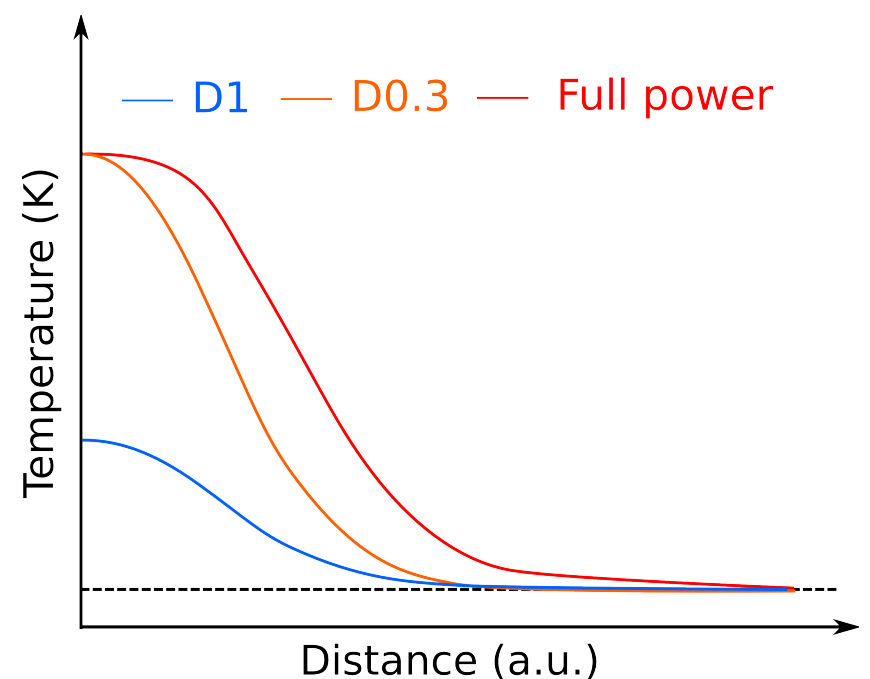

Figure 9.15: Spatial temperature distribution at D1, D0.3 and full laser powers. The black dashed line represents $300 \mathrm{~K}$.

curve in Figure 9.15). Spectra collected at lower laser power have larger regions at a uniform temperature of $300 \mathrm{~K}$ as well as temperatures lower than the saturation temperature. The physical mechanisms behind the rise in ambient temperature and the saturation temperature have been discussed in terms of nanowire coverage and cooling mechanisms respectively. 


\section{Chapter 10}

\section{Conclusions}

This thesis looked at the Raman and conduction characterisitics of two types of nanomaterials: SWNT networks and cupric oxide nanowires. The key findings are summarised by material.

\section{SWNT networks}

1. The resistance of SWNT networks can be controlled by the amount of deposited nanotubes based on a percolation argument. Larger density of tubes implies better/more conduction pathways which bring down the resistance. We have demonstrated a fabrication setup using an ultrasonic nozzle to spray an ink of SWNTs (dispersed in volatile solvents) on a heated substrate. The spray descends as a wet aerosol but forms a dry coating on the substrate as the solvents evaporate on contact. Thus, the resistance of this deposited dry material can be used to monitor film deposition, allowing the user to fabricate networks of the desired resistance based on deposition time alone. The monitored resistance can be analysed with reference to the percolation model.

2. Spatially averaged Raman spectra of the 'dry-sprayed samples showed that our fabrication process does not appear to chemically dope or alter the samples significantly. Thus, we were able to fabricate pristine SWNT networks with resistances between $300 \mathrm{k} \Omega$ and $8 \mathrm{k} \Omega$.

3. The spatial homogeneity of the networks was characterised from spatially-sensitive optical transparency measurements. This was achieved with the spatial mapping stage of a Raman spectrometer and a power meter. The micrometer sensitivity of this optical data indicated that the ultrasonic nozzle could spray highly homogeneous networks as well as networks with a large spatial variation in sample density. Interestingly, no correlation between homogeneity and sample resistance was found in the tested samples. 


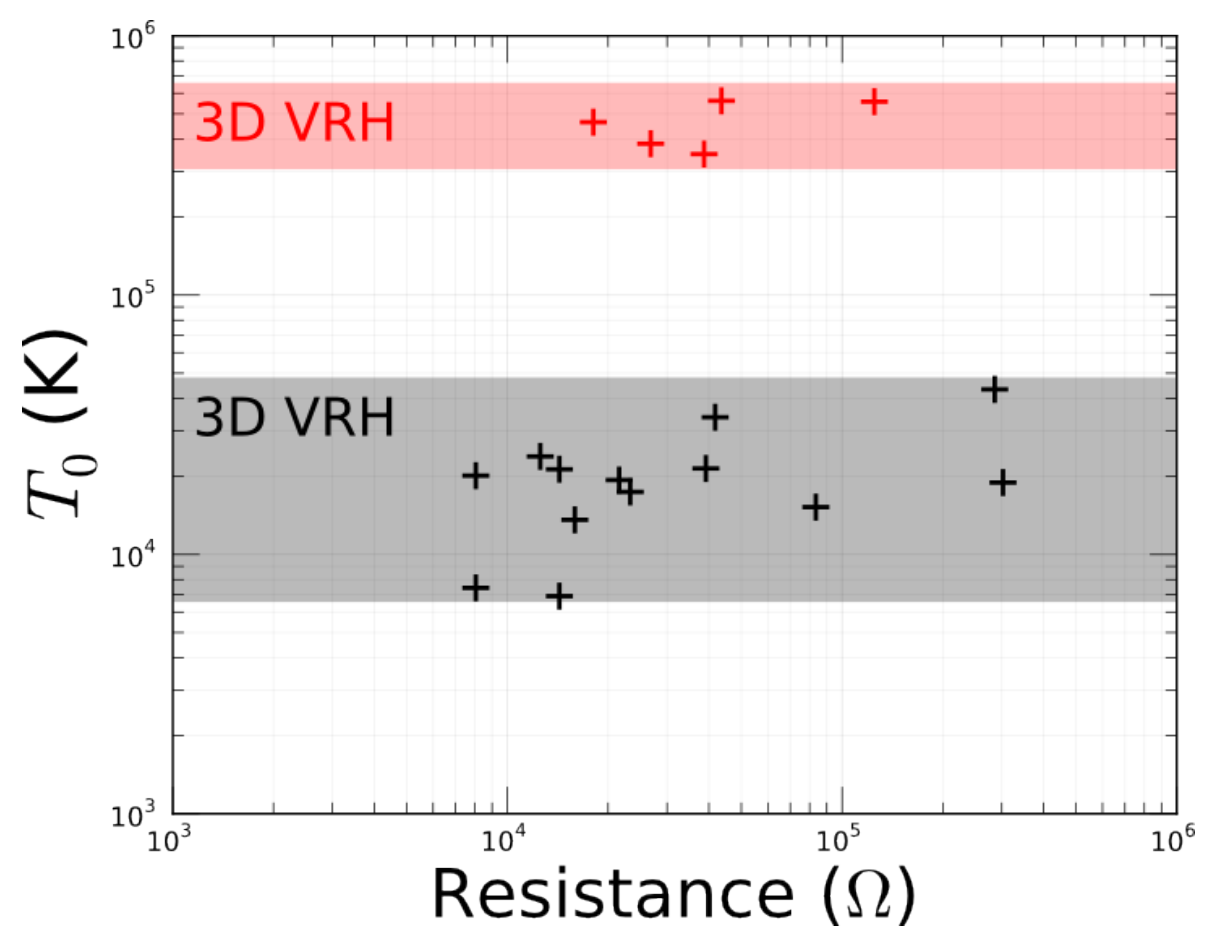

Figure 10.1: $T_{0}$ vs. sample resistance.

4. Charge transport mechanisms were identified from the temperature-dependent resistance of the sprayed samples. Despite the large variation in resistances, a characteristic charge transport model could be used to describe the majority of the samples. The resistance below $220 \mathrm{~K}$ (to $\sim 20-50 \mathrm{~K}$ ) could be described by 2D VRH with a barrier size of 20,000 $\pm 9400 \mathrm{~K}$. Above $220 \mathrm{~K}$, a thermal activation term with an activation energy of $160 \pm 20$ meV supplemented the VRH mechanism. Samples that could be described by 2D VRH were described by 3D VRH. Again, with a characteristic barrier size and an activation energy of $160 \mathrm{meV}$.

5. The spatial homogeneity measurements indicate a correlation between 3D VRH and inhomogeneous samples. Finally, we note that despite resistances as low as $8 \mathrm{k} \Omega$, conducting pathways in the SWNT network are dominated by non-metallic tubes.

6. Aside from studying the fundamental transport characteristics in pristine SWNT networks, we have also explored the vibrational and optical characteristics using Raman spectroscopy. Raman spectroscopy of SWNT networks is a poorly-studied field, particularly for the radial breathing modes (RBMs). This spectral region becomes increasingly complicated due to the the number of component peaks and, poor resolution of a given peak. Thus, in order to study RBMs in SWNT networks, we developed a sophisticated, high resolution fitting routine. The routine uses higher order derivatives to find and fit finer features that cannot be seen clearly in the original spectra. 
7. Using this fitting procedure, we studied the temperature dependence of the RBM intensity, peak frequency and linewidth from bundled SWNT regions within the network. Our results indicate that peak frequencies in these bundled regions soften similarly to individual SWNTs; linewidths can be described by a three phonon decay process along with a temperature-independent linewidth of $4.6 \mathrm{~cm}^{-1}$ and the RBM intensity can be modelled according to the temperature-dependent changes in the electronic transition energies $\left(E_{i i}\right)$.

\section{Cupric oxide}

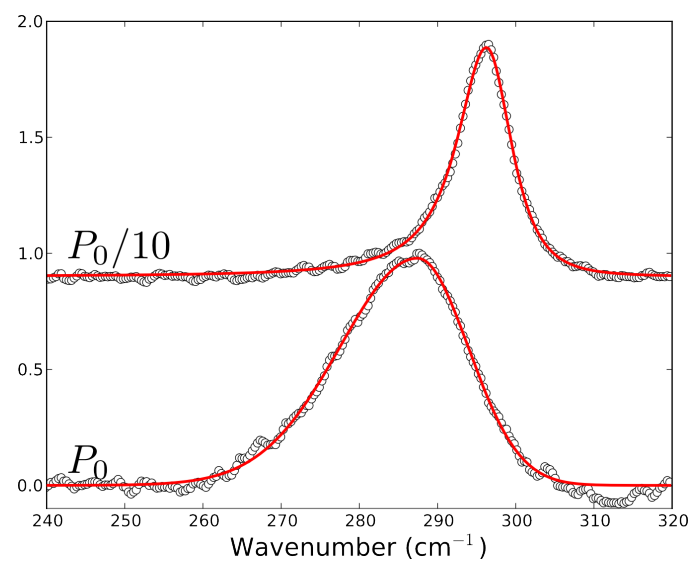

(a) $A_{1 g}$ mode in thin film

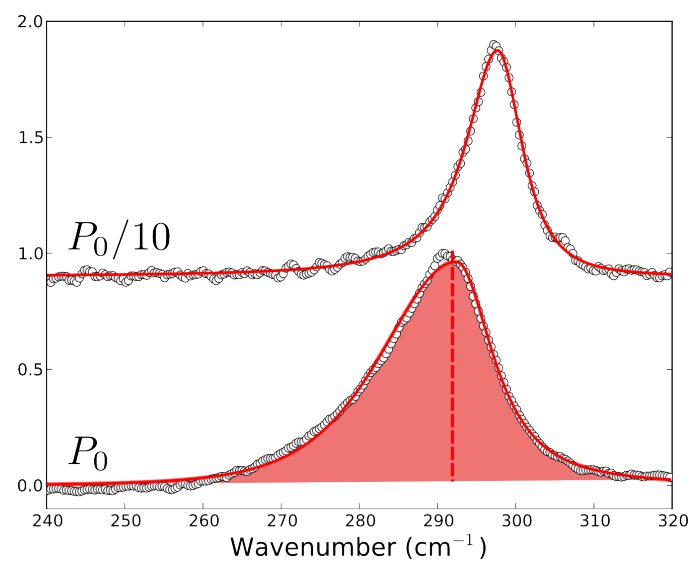

(b) $A_{1 g}$ mode in nanowire forest

Figure 10.2: Laser power dependence of $A_{1 g}$ mode in a thin film (a) and a nanowire forest (b).

1. The second strand of this $\mathrm{PhD}$ work was motivated by the power-dependent lineshape asymmetry of the $A_{1 g}$ mode in $\mathrm{CuO}$ nanowire forests (Figure 10.2). The power dependence implied a thermal phenomenon due to the laser power. Compared to the symmetric broadening in the thin film, due to uniform increase in local temperature, the asymmetric broadening is a result of spatial temperature gradients.

2. The temperature dependence of the $A_{1 g}$ mode in 'bulk' $\mathrm{CuO}$ was studied in order to model the effects in the nanowire forest.

3. The bulk study showed that the decreasing Stokes signal with increasing temperature is an integral component behind the asymmetry. This finding allowed for a simple model based on an inhomogeneous temperature distribution induced by a gaussian laser beam. The induced temperature distribution can be described by two characteristic temperatures: $T_{a}$ and $T_{\max }$. As a simple approximation, the signal from the heated forest can be considered a superposition of signal from temperature regions between $T_{\max }$ and $T_{a}$. This model gives the correct lineshape asymmetry because the higher the temperature, the lower the signal, despite the fact that regions at high temperature receive the largest 
photon flux. This simple model was modified slightly to include a region at uniform temperature to better fit the reality of induced temperatures in the nanowire forest.

4. The fitted values of $T_{a}$ and $T_{\max }$ show that the maximum temperature actually saturates to a limiting value at $50 \%$ of full power. This saturation combined with a gradually increasing value of $T_{a}$ has the effect of reducing the lineshape asymmetry. Destruction of nanowires by melting or decomposition may be ruled out as the mechanism behind the saturation temperature. Instead, we speculate that a change in cooling mechanism: from convection cooling to radiation, occurs around $T_{\max }$. The increase in the minimum observed temperature, or $T_{a}$, is attributed to variable nanowire coverage. 


\section{Chapter 11}

\section{Appendix 1}

\section{Airbrushing}

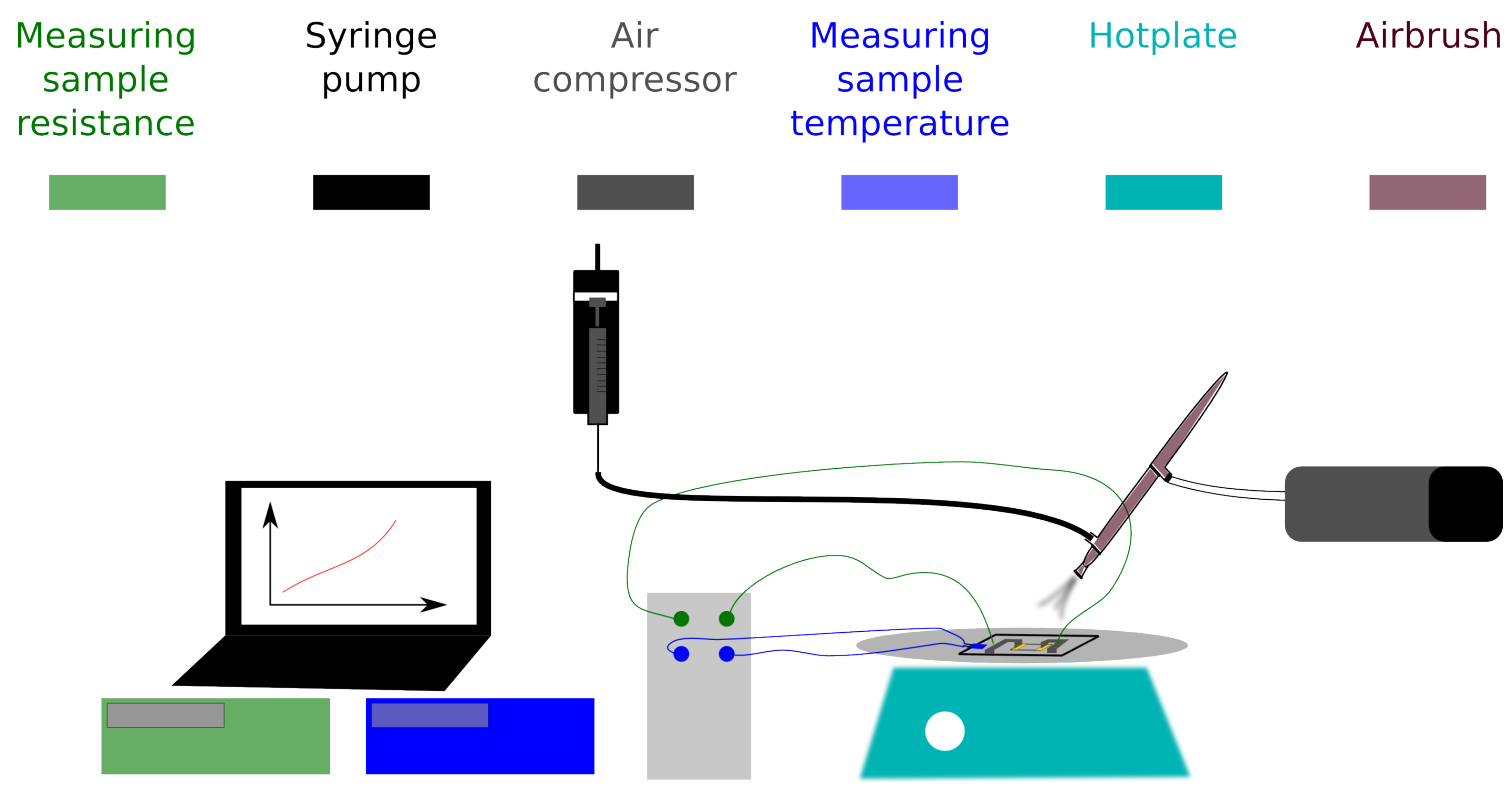

Figure 11.1: A simple schematic showing the initial experimental set up (with a hobby airbrush) for in-situ monitoring of SWNT network conductance.

The deposition apparatus with which consistent success was achieved is shown in Figure 4.4 . However, the route to this final setup required some optimisation. The initial setup consisted of an airbrush and compressor (Figure 11.1) instead of an ultrasonic nozzle. The airbrush sprayed the solution, injected at constant speed into the feed hole, onto the substrate below using air pumped from a small compressor (at $\sim 5 \mathrm{psi}$ ). The angle and height of the spray could be adjusted for homogeneous coating of the substrate. The substrate, mounted on a metallic clamp, was a glass coverslip $(12 \mathrm{~mm} \times 12 \mathrm{~mm})$ with $2 \mathrm{~mm}$ wide patterned $\mathrm{Cr} / \mathrm{Au}$ electrodes. The electrodes were contacted using a mechanical clamp for two reasons: (i) to avoid solder/indium flow onto the samples if the substrate is heated above $\sim 150{ }^{\circ} \mathrm{C}$ and (ii) to secure the light, glass substrate from the force of the spray. Further details of fabrication can be found in Chapter 4.2 .2 . 
A small number of datasets showing the evolution of the sample conductance as a function of deposition time were obtained with this set-up. One such dataset is analysed in Figure 11.2. The percolation threshold, $\sim 232 \mathrm{~s}$, can be extracted by plotting the conductance data on a log scale, as seen in Figure 11.2a. The threshold-corrected data can then be plotted to identify the different regimes namely, the power law and linear regimes. The log plot in Figure $11.2 \mathrm{~b}$, however, shows three power law regimes. Linear fits for each regime, where the gradient gives $\alpha$ and the exponential of the intercept gives $G_{0}$, are superimposed on the full trace. The first region is a short-lived sub-linear regime that only appears for the initial $15 \mathrm{~s}$ after percolation is established. This region may correspond to sub-percolation [63]. The second regime dominates for $550 \mathrm{~s}$ and is described by an exponent of 2.38 - much higher than that expected from 3D percolation (1.93). The third regime is super-linear - described by an exponent of 1.65 - rather than the expected linear trend.

Typically, theoretical values of $\alpha-1.94$ (3D percolation) and 1.33 (2D percolation) - do not match the values found in the existing literature for SWNT films. $\alpha$ values fitted to experimental data range between the values of 1.94 and 1.33; a discrepancy that has been addressed as "some crossover into three dimensions" [47]. This crossover could be related to the length distribution of the SWNTs, where networks comprised of short or mixed-length tubes tend towards 3D percolation while networks of long tubes demonstrate 2D percolation [40]. However, in the case of spraying, the percolation trace can be coupled with the quality and the speed of deposition. When the deposition rate is high, the power law reflects this feature rather than the percolation model. This dependence on the spray characteristics is the likely reason behind the large values of $\alpha$ in both the percolation and linear regimes.

This initial set-up (with a hobby grade airbrush) was chosen for its low cost so that experiment of in-situ deposition could get underway. The experiment was successful but there were several problems: surface cooling, reproducibility and mechanical damage to the contacts. Strong surface cooling of the substrate by air pressure from the carrier gas required higher heating temperatures than desirable. The surface temperature was almost $100{ }^{\circ} \mathrm{C}$ cooler under the spray and further lowered by $\sim 170{ }^{\circ} \mathrm{C}$ due to poor thermal contact between the clamp and the substrate. The total thermal load from the heater to the substrate surface was $500{ }^{\circ} \mathrm{C}$ (this also included the small thermal resistance between the clamp and the heater). So, for a substrate surface temperature of $\sim 100{ }^{\circ} \mathrm{C}$, the heater had to be maintained at $650{ }^{\circ} \mathrm{C}$. Reproducibility was hindered by poor control of the spray area and frequent blocking of the nozzle due to the prolonged collection of nanotube clumps. This latter issue was a consequence of the high volume of solution passing through the nozzle - the spray cone of the airbrush was quite large with diameters around $50 \mathrm{~mm}$. The final, and most relevant, issue was the mechanical damage caused to the electrodes due to the mechanical clamp. Damage to the contacts during deposition not only affected the accuracy of the percolation trace but also prevented the sample from being used for other measurements. 


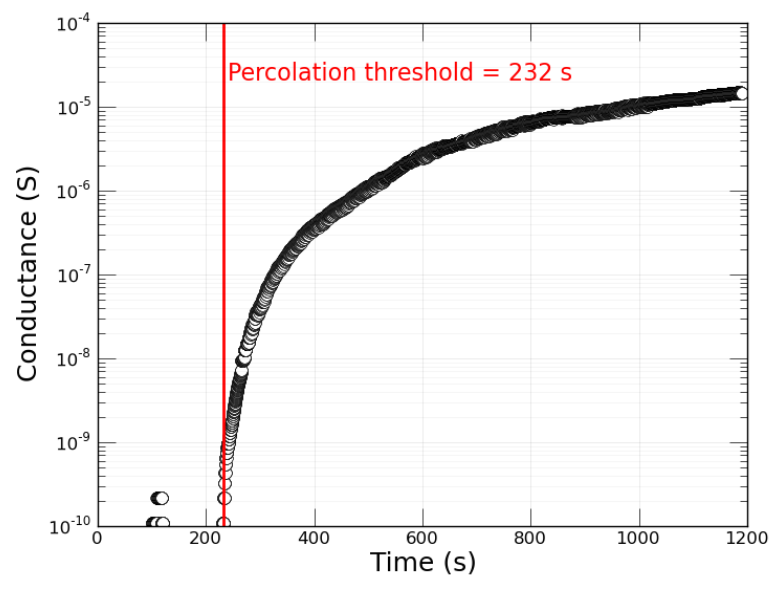

(a) Percolation threshold

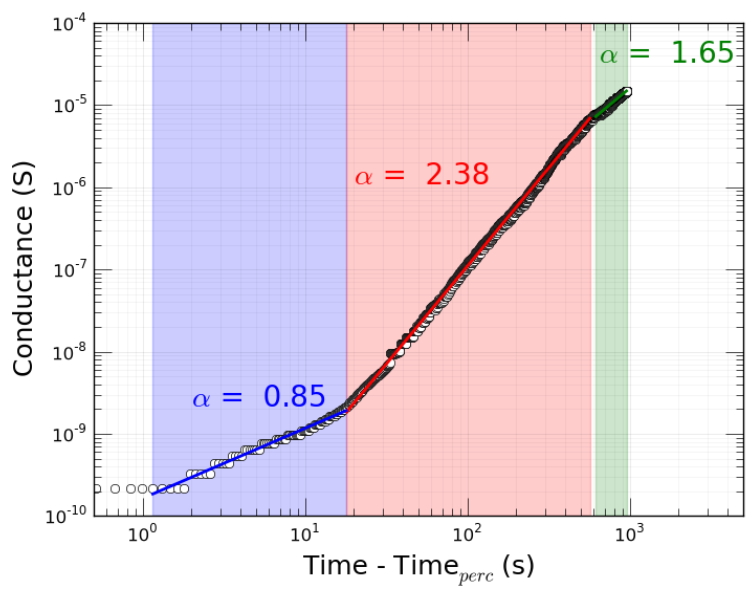

(b) Log plot of the full trace

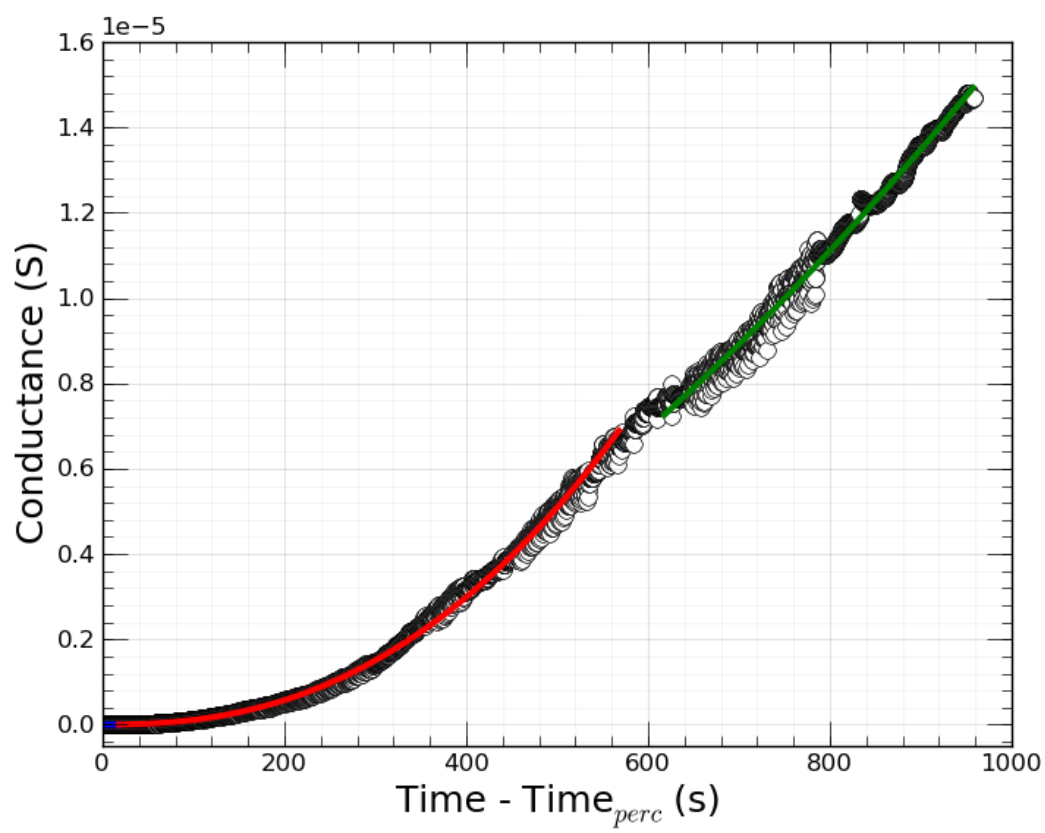

(c) Linear plot of the full trace

Figure 11.2: (a) Determining the percolation threshold from a semi-log plot. (b) Three power law regimes. (c) Data and fits on a linear scale showing the distinct power law and super-linear regimes. 


\section{Chapter 12}

\section{Appendix 2}

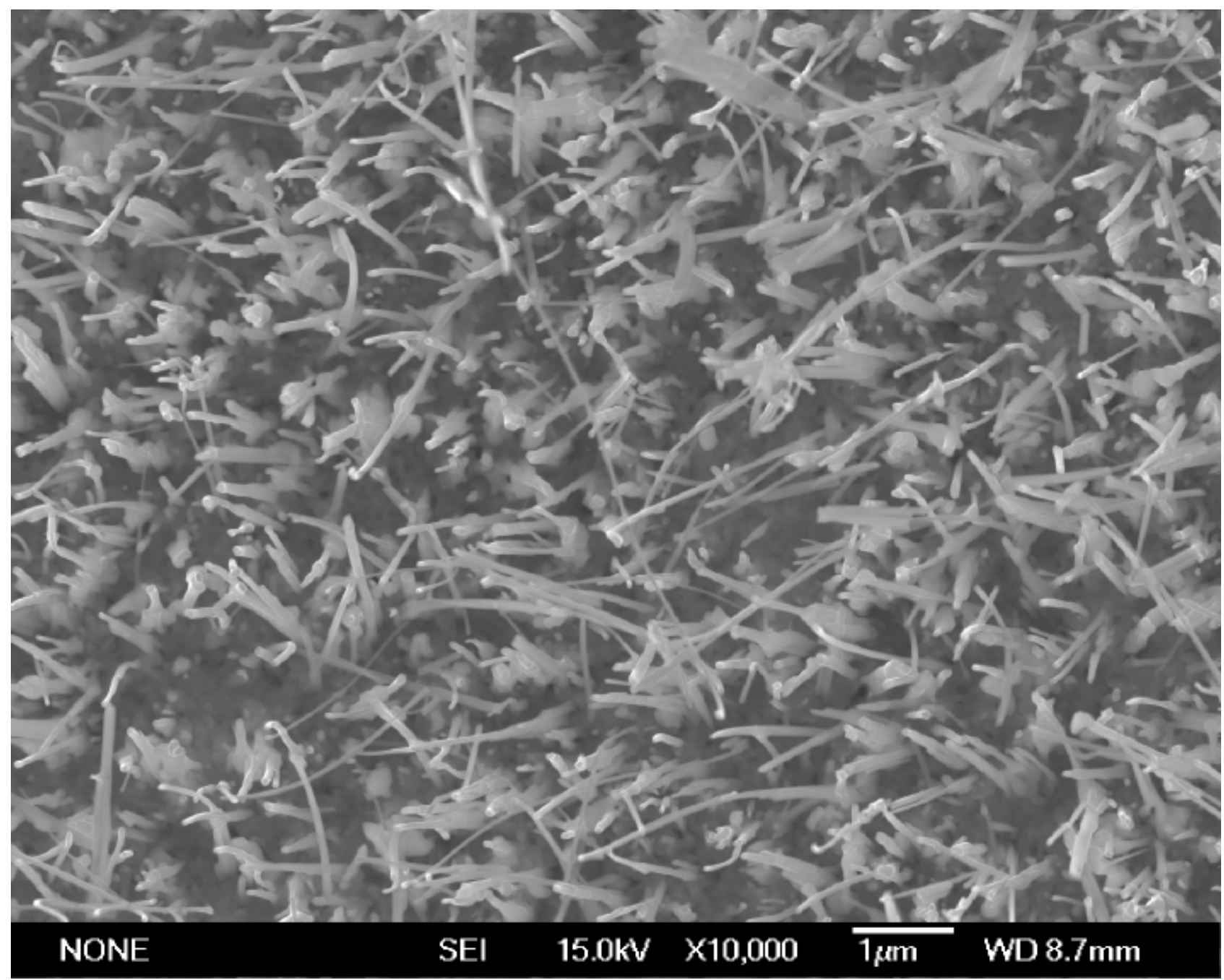

Figure 12.1

Figure 12.2: Irradiated sample. 


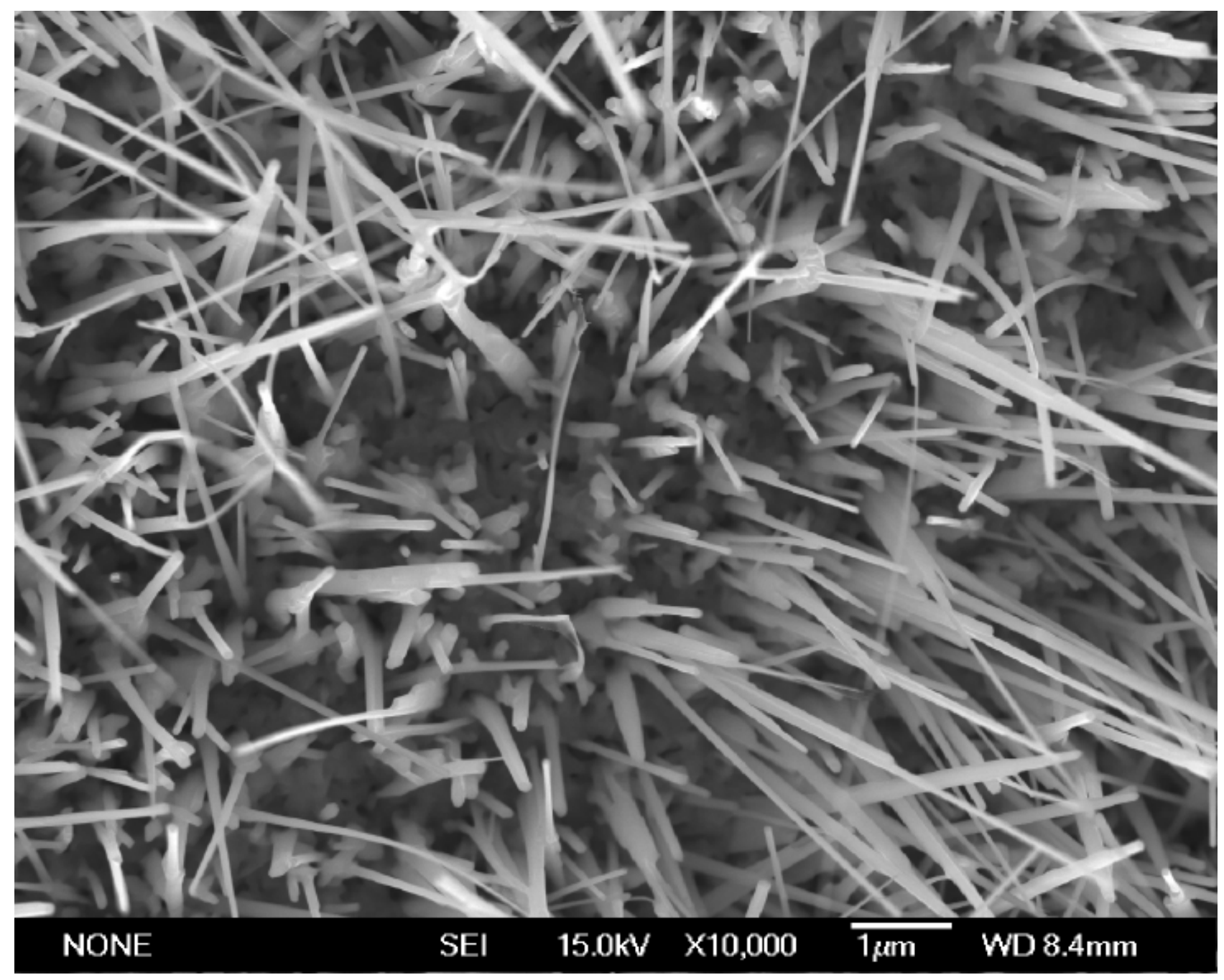

Figure 12.3: As-prepared $\mathrm{CuO}$ nanowire forest courtesy of James Eldridge. 


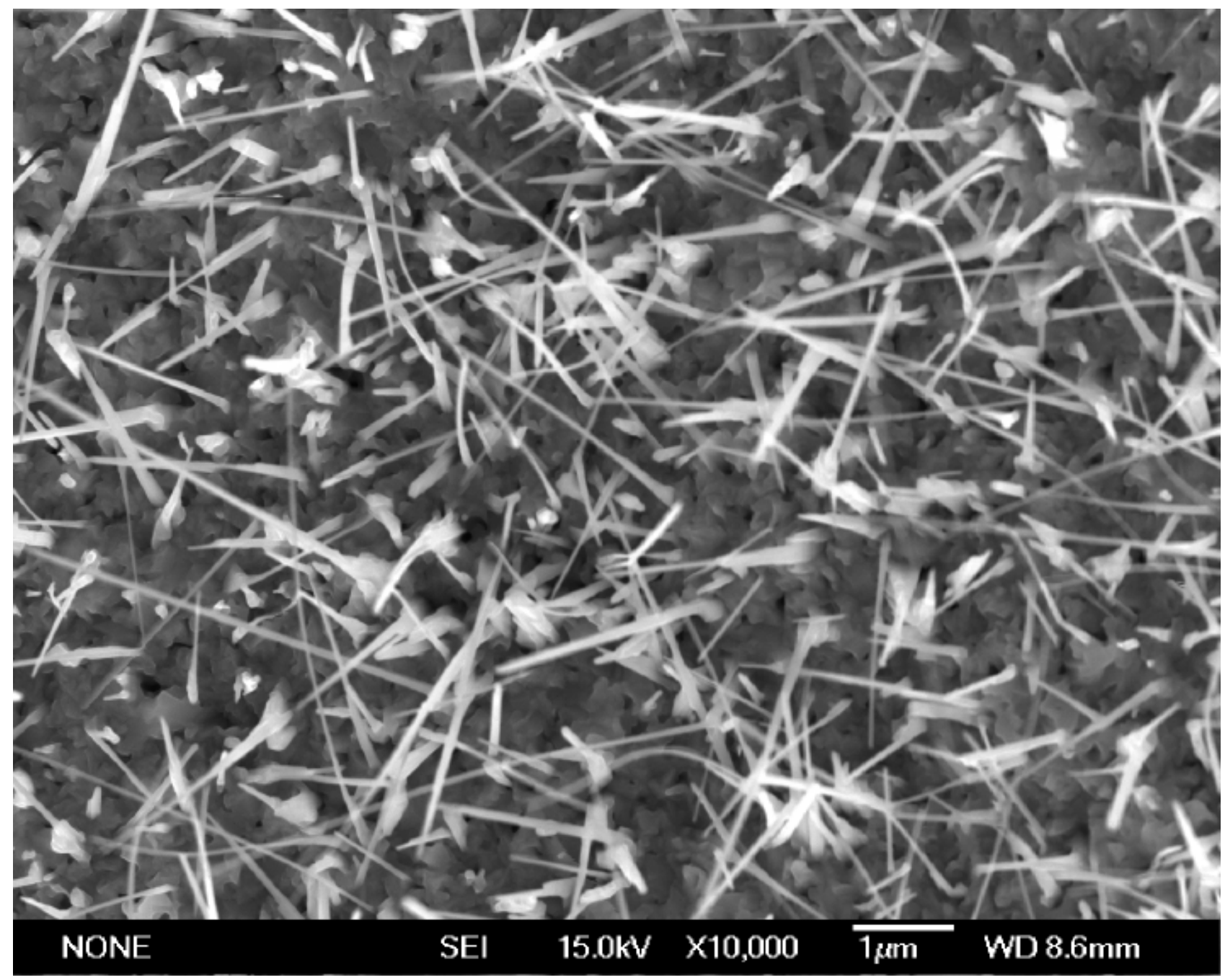

Figure 12.4: As-prepared $\mathrm{CuO}$ nanowire forest courtesy of James Eldridge. 


\section{Bibliography}

[1] J. Ziman, "Electrons and phonons: The theory of transport phenomena in solids," 2001.

[2] P. Sheng, E. K. Sichel, and J. I. Gittleman, "Fluctuation-induced tunneling conduction in carbon-polyvinylchloride composites," Physical Review Letters, vol. 40, no. 18, pp. 11971200, 1978.

[3] N. F. Mott, "Conduction in non-crystalline materials," Philosophical Magazine, vol. 19, no. 160, pp. 835-852, 1969.

[4] C. V. Raman and K. S. Krishnan, "A new type of secondary radiation," Nature, vol. 121, no. 3048, pp. 501-502, 1928.

[5] C. V. Raman and K. S. Krishnan, "The optical analogue of the compton effect," Nature, vol. 121, no. 3053, pp. 711-711, 1928.

[6] G. Lucazeau, "Effect of pressure and temperature on raman spectra of solids: anharmonicity," Journal of Raman Spectroscopy, vol. 34, no. 7-8, pp. 478-496, 2003.

[7] R. S. Krishnan, "The second order raman spectrum of diamond," in Proceedings of the Indian Academy of Sciences, Section A, vol. 24, pp. 25-32, Indian Academy of Sciences, 1946.

[8] P. G. Klemens, "Anharmonic decay of optical phonons," Physical Review, vol. 148, pp. 845-848, Aug 1966.

[9] W. Demtröder, "Laser spectroscopy," 2003.

[10] H. D. Li, K. Yue, Z. L. Lian, Y. Zhan, L. X. Zhou, S. L. Zhang, Z. J. Shi, Z. N. Gu, B. B. Liu, R. S. Yang, H. B. Yang, G. T. Zou, Y. Zhang, and S. Iijima, "Temperature dependence of the raman spectra of single-wall carbon nanotubes," Applied Physics Letters, vol. 76 , no. 15 , pp. 2053-2055, 2000.

[11] M. Balkanski, R. F. Wallis, and E. Haro, "Anharmonic effects in light scattering due to optical phonons in silicon," Physical Review B, vol. 28, no. 4, pp. 1928-1934, 1983.

[12] Y. Kim, X. Chen, Z. Wang, J. Shi, I. Miotkowski, Y. P. Chen, P. A. Sharma, A. L. Lima Sharma, M. A. Hekmaty, Z. Jiang, and D. Smirnov, "Temperature dependence of 
raman-active optical phonons in bi2se3 and sb2te3," Applied Physics Letters, vol. 100, no. 7, pp. 071907-071907, 2012.

[13] H. Tang and I. Herman, "Raman microprobe scattering of solid silicon and germanium at the melting temperature," Physical Review B, vol. 43, no. 3, p. 2299, 1991.

[14] Y. S. Raptis, G. A. Kourouklis, E. Anastassakis, E. Haro-Poniatowski, and M. Balkanski, "Anharmonic effects in mg2x ( $\mathrm{x}=\mathrm{si}$, ge, sn) compounds studied by raman spectroscopy," Journal de Physique, vol. 48, no. 2, pp. 239-245, 1987.

[15] H. Herchen and M. A. Cappelli, "First-order raman spectrum of diamond at high temperatures," Physical Review B, vol. 43, pp. 11740-11744, May 1991.

[16] I. P. Herman, "Peak temperatures from raman stokes/anti-stokes ratios during laser heating by a gaussian beam," Journal of Applied Physics, vol. 109, no. 1, p. 016103, 2011.

[17] A. Compaan and H. J. Trodahl, "Resonance raman scattering in si at elevated temperatures," Physical Review B, vol. 29, no. 2, p. 793, 1984.

[18] O. Chaix-Pluchery, L. Abello, G. Lucazeau, B. Chenevier, and R. Madar, "A raman study of single crystal and thin film tetragonal wsi 2 ," Journal of Physics and Chemistry of Solids, vol. 57, no. 5, pp. 527-537, 1996.

[19] G. E. Jellison and F. A. Modine, "Optical functions of silicon between 1.7 and 4.7 ev at elevated temperatures," Physical Review B, vol. 27, pp. 7466-7472, Jun 1983.

[20] M. Lax, "Temperature rise induced by a laser beam," Journal of Applied Physics, vol. 48, no. 9, pp. 3919-3924, 1977.

[21] F. Marabelli, G. Parravicini, and F. Salghetti-Drioli, "Optical gap of cuo," Physical Review $B$, vol. 52, pp. 1433-1436, 1995.

[22] A. Rakhshani and F. Barakat, "Optical constants of reactively sputtered cupric oxide films," Materials Letters, vol. 6, no. 1-2, pp. 37-40, 1987.

[23] T. Tanaka, "Optical constants of polycrystaffine 3d transition metal oxides in the wavelength region 350 to 1200 nm," Japanese Journal of Applied Physics, vol. 18, no. 6, pp. 1043-1047, 1979.

[24] E. C. Le Ru, E. Blackie, M. Meyer, and P. G. Etchegoin, "Surface enhanced raman scattering enhancement factors: a comprehensive study," The Journal of Physical Chemistry C, vol. 111, no. 37, pp. 13794-13803, 2007.

[25] G. Grüner, "Carbon nanotube films for transparent and plastic electronics," Journal of Materials Chemistry, vol. 16, no. 35, pp. 3533-3539, 2006.

[26] D. Stauffer and A. Aharony, Introduction to percolation theory. CRC, 1994. 
[27] D. Hecht and G. Grüner, "Solution Cast Films of Carbon Nanotubes for Transparent Conductors and Thin Film Transistors," Flexible Electronics: Materials and Applications, p. 297, 2009.

[28] A. B. Kaiser, G. Düsberg, and S. Roth, "Heterogeneous model for conduction in carbon nanotubes," Physical Review B, vol. 57, no. 3, pp. 1418-1421, 1998.

[29] P. N. Nirmalraj, P. E. Lyons, S. De, J. N. Coleman, and J. J. Boland, "Electrical Connectivity in Single-Walled Carbon Nanotube Networks," Nano Letters, vol. 11, pp. 38903895, 2009.

[30] Y. H. Lin, K. J. Lin, F. R. Chen, J. J. Kai, and J. J. Lin, "Electronic Transport Through Metal Nanowire Contacts," Nanoscale Phenomena, pp. 139-148.

[31] N. F. Mott and E. A. Davis, Electronic processes in non-crystalline materials. Clarendon Press Oxford, 1971.

[32] V. Ambegaokar, B. I. Halperin, and J. S. Langer, "Hopping conductivity in disordered systems," Physical Review B, vol. 4, no. 8, pp. 2612-2620, 1971.

[33] J. J. Hauser and A. Staudinger, "Electrical and structural properties of amorphous germanium," Physical Review B, vol. 8, no. 2, p. 607, 1973.

[34] http://xdb.lbl.gov/, "X-ray data booklet,"

[35] R. W. Cheary and J. P. Cline, "An analysis of the effect of different instrumental conditions on the shapes of x-ray powder line profiles," Advances in X-ray analysis, vol. 38, no. CONF-9408178-, pp. 75-82, 1995.

[36] P. P. Edwards, A. Porch, M. O. Jones, D. V. Morgan, and R. M. Perks, "Basic materials physics of transparent conducting oxides," Dalton transactions, vol. 2004, no. 19, pp. 2995-3002, 2004.

[37] R. J. Brown, "Indium," USGS Mineral Commodity Summaries, 1991-2010.

[38] M. G. Kang and L. J. Guo, "Nanoimprinted semitransparent metal electrodes and their application in organic light-emitting diodes," Advanced Materials, vol. 19, p. 1391, 2007.

[39] J. Y. Lee, S. T. Connor, Y. Cui, and P. Peumans, "Solution-processed metal nanowire mesh transparent electrodes," Nano Letters, vol. 8, no. 2, pp. 689-692, 2008.

[40] D. Simien, J. A. Fagan, W. Luo, J. F. Douglas, K. Migler, and J. Obrzut, "Influence of Nanotube Length on the Optical and Conductivity Properties of Thin Single-Wall Carbon Nanotube Networks," ACS nano, vol. 2, no. 9, pp. 1879-1884, 2008.

[41] S. Kumar, J. Y. Murthy, and M. A. Alam, "Percolating conduction in finite nanotube networks," Physical review letters, vol. 95, no. 6, pp. 066802-066805, 2005. 
[42] C. Journet, W. K. Maser, P. Bernier, A. Loiseau, M. Lamy de La Chapelle, S. Lefrant, P. Deniard, R. Lee, and J. E. Fischer, "Large-scale production of single-walled carbon nanotubes by the electric-arc technique," Nature, vol. 388, no. 6644, pp. 756-757, 1997.

[43] E. M. Doherty, S. De, P. E. Lyons, A. Shmeliov, P. N. Nirmalraj, V. Scardaci, J. Joimel, W. J. Blau, J. J. Boland, and J. N. Coleman, "The spatial uniformity and electromechanical stability of transparent, conductive films of single walled nanotubes," Carbon, vol. 47, no. 10, pp. 2466-2473, 2009.

[44] "http://www.carbonsolution.com/faq.html,"

[45] Y. Zhou, L. Hu, and G. Grüner, "A method of printing carbon nanotube thin films," Applied physics letters, vol. 88, p. 123109, 2006.

[46] B. Ruzicka, L. Degiorgi, R. Gaal, L. Thien-Nga, R. Bacsa, J. Salvetat, and L. Forro, "Optical and dc conductivity study of potassium-doped single-walled carbon nanotube films," Physical Review B, vol. 61, no. 4, pp. 2468-2471, 2000.

[47] L. Hu, D. Hecht, and G. Grüner, "Percolation in transparent and conducting carbon nanotube networks," Nano Letters, vol. 4, no. 12, pp. 2513-2517, 2004.

[48] C. T. White and T. N. Todorov, "Carbon nanotubes as long ballistic conductors," Nature, vol. 393, no. 6682, pp. 240-242, 1998.

[49] K. Yanagi, H. Udoguchi, S. Sagitani, Y. Oshima, T. Takenobu, H. Kataura, T. Ishida, K. Matsuda, and Y. Maniwa, "Transport mechanisms in metallic and semiconducting single-wall carbon nanotube networks," ACS Nano, vol. 4, no. 7, pp. 4027-4032, 2010.

[50] T. Barnes, J. Blackburn, J. van de Lagemaat, T. Coutts, and M. Heben, "Reversibility, dopant desorption, and tunneling in the temperature-dependent conductivity of typeseparated, conductive carbon nanotube networks.," ACS nano, vol. 2, no. 9, p. 1968, 2008.

[51] M. A. Topinka, M. W. Rowell, D. Goldhaber-Gordon, M. D. McGehee, D. S. Hecht, and G. Grüner, "Charge Transport in Interpenetrating Networks of Semiconducting and Metallic Carbon Nanotubes," Nano letters, vol. 9, no. 5, pp. 1866-1871, 2009.

[52] S. Ravi, A. B. Kaiser, and C. W. Bumby, "Improved conduction in transparent single walled carbon nanotube networks drop-cast from volatile amine dispersions," Chemical Physics Letters, vol. 496, no. 1-3, pp. 80-85, 2010.

[53] H. Z. Geng, K. K. Kim, C. Song, N. T. Xuyen, S. M. Kim, K. A. Park, D. S. Lee, K. H. An, Y. S. Lee, Y. Chang, A. Benayad, and H. L. Young, "Doping and de-doping of carbon nanotube transparent conducting films by dispersant and chemical treatment," J. Mater. Chem., vol. 18, no. 11, pp. 1261-1266, 2008. 
[54] V. Skákálova, A. B. Kaiser, U. Dettlaff-Weglikowska, K. Hrncarikova, and S. Roth, "Effect of chemical treatment on electrical conductivity, infrared absorption, and Raman spectra of single-walled carbon nanotubes," J. Phys. Chem. B, vol. 109, no. 15, pp. 7174-7181, 2005 .

[55] S. Reich, C. Thomsen, and J. Maultzsch, Carbon nanotubes: basic concepts and physical properties. Vch Verlagsgesellschaft Mbh, 2004.

[56] R. Saito, G. Dresselhaus, and M. S. Dresselhaus, Physical properties of carbon nanotubes, vol. 4. Imperial college press London, 1998.

[57] E. Richter and K. R. Subbaswamy, "Theory of size-dependent resonance raman scattering from carbon nanotubes," Physical review letters, vol. 79, no. 14, pp. 2738-2741, 1997.

[58] "http://www.photon.t.u-tokyo.ac.jp/ maruyama/kataura/kataura.html,"

[59] A. Kukovecz, C. Kramberger, V. Georgakilas, M. Prato, and H. Kuzmany, "A detailed raman study on thin single-wall carbon nanotubes prepared by the hipco process," The European Physical Journal B-Condensed Matter and Complex Systems, vol. 28, no. 2, pp. 223-230, 2002.

[60] M. Strano, "Probing chiral selective reactions using a revised Kataura plot for the interpretation of single-walled carbon nanotube spectroscopy," Journal of the American Chemical Society, vol. 125, no. 51, pp. 16148-16153, 2003.

[61] A. V. Naumov, O. A. Kuznetsov, A. R. Harutyunyan, A. A. Green, M. C. Hersam, D. E. Resasco, P. N. Nikolaev, and R. B. Weisman, "Quantifying the Semiconducting Fraction in Single-Walled Carbon Nanotube Samples through Comparative Atomic Force and Photoluminescence Microscopies," Nano Letters, vol. 9, no. 9, pp. 3203-3208, 2009.

[62] S. Paul and D. W. Kim, "Preparation and characterization of highly conductive transparent films with single-walled carbon nanotubes for flexible display applications," Carbon, 2009 .

[63] H. E. Unalan, G. Fanchini, A. Kanwal, A. Du Pasquier, and M. Chhowalla, "Design criteria for transparent single-wall carbon nanotube thin-film transistors," Nano Letters, vol. 6, no. 4, pp. 677-682, 2006.

[64] A. Schindler, J. Brill, N. Fruehauf, J. P. Novak, and Z. Yaniv, "Solution-deposited carbon nanotube layers for flexible display applications," Physica E: Low-dimensional Systems and Nanostructures, vol. 37, no. 1-2, pp. 119-123, 2007.

[65] P. G. Collins, M. S. Arnold, and P. Avouris, "Engineering carbon nanotubes and nanotube circuits using electrical breakdown," Science, vol. 292, no. 5517, pp. 706-709, 2001.

[66] M. Dressel and G. Grüner, Electrodynamics of solids: optical properties of electrons in matter. Cambridge University Press, 2002. 
[67] S. De and J. N. Coleman, "The effects of percolation in nanostructured transparent conductors," MRS Bulletin, vol. 36, no. 10, pp. 774-781, 2011.

[68] P. G. Collins, K. Bradley, M. Ishigami, and A. Zettl, "Extreme oxygen sensitivity of electronic properties of carbon nanotubes," Science, vol. 287, no. 5459, p. 1801, 2000.

[69] R. K. Jackson, A. Munro, K. Nebesny, N. Armstrong, and S. Graham, "Evaluation of transparent carbon nanotube networks of homogeneous electronic type," ACS Nano, vol. 4, no. 3, pp. 1377-1384, 2010.

[70] H. Geng and Y. Lee, "Transparent conducting films by using carbon nanotubes," Nanoscale Phenomena, pp. 15-28.

[71] H. Geng, K. Kim, K. So, Y. Lee, Y. Chang, Y. Lee, et al., "Effect of acid treament on carbon nanotube-based flexible transparent conducting films," Journal of the American Chemical Society, vol. 129, no. 25, pp. 7758-7759, 2007.

[72] J. Blackburn, T. Barnes, M. Beard, Y. Kim, R. Tenent, T. McDonald, B. To, T. Coutts, and M. Heben, "Transparent Conductive Single-Walled Carbon Nanotube Networks with Precisely Tunable Ratios of Semiconducting and Metallic Nanotubes," ACS nano, vol. 2, no. 6, pp. 1266-1274, 2008.

[73] H. Z. Geng, D. S. Lee, K. K. Kim, G. H. Han, H. K. Park, and Y. H. Lee, "Absorption spectroscopy of surfactant-dispersed carbon nanotube film: modulation of electronic structures," Chemical Physics Letters, vol. 455, no. 4-6, pp. 275-278, 2008.

[74] S. Suzuki, Y. Watanabe, Y. Homma, S. Fukuba, S. Heun, and A. Locatelli, "Work functions of individual single-walled carbon nanotubes," Applied Physics Letters, vol. 85, p. 127, 2004.

[75] V. Skákalová, A. Kaiser, Y. Woo, and S. Roth, "Electronic transport in carbon nanotubes: From individual nanotubes to thin and thick networks," Physical Review B, vol. 74, no. 8, p. 85403, 2006.

[76] C. Fantini, A. Jorio, M. Souza, M. S. Strano, M. S. Dresselhaus, and M. A. Pimenta, "Optical transition energies for carbon nanotubes from resonant Raman spectroscopy: Environment and temperature effects," Physical Review letters, vol. 93, no. 14, p. 147406, 2004.

[77] Q. Cheng, S. Debnath, E. Gregan, and H. J. Byrne, "Effect of solvent solubility parameters on the dispersion of single-walled carbon nanotubes," The Journal of Physical Chemistry C, vol. 112, no. 51, pp. 20154-20158, 2008.

[78] M. S. Strano, V. . Moore, M. K. Miller, M. J. Allen, E. H. Haroz, C. Kittrell, R. H. Hauge, and R. E. Smalley, "The role of surfactant adsorption during ultrasonication in the dispersion of single-walled carbon nanotubes," Langmuir, vol. 18, p. 2673, 2002. 
[79] M. J. O'connell, S. M. Bachilo, C. B. Huffman, V. C. Moore, M. S. Strano, E. H. Haroz, K. L. Rialon, P. J. Boul, W. H. Noon, C. Kittrell, J. Ma, R. Haugel, R. B. Weisman, and R. E. Smalley, "Band gap fluorescence from individual single-walled carbon nanotubes," Science, vol. 297, no. 5581, pp. 593-596, 2002.

[80] O. K. Park, T. Jeevananda, N. H. Kim, S. Kim, and J. H. Lee, "Effects of surface modification on the dispersion and electrical conductivity of carbon nanotube/polyaniline composites," Scripta Materialia, vol. 60, no. 7, pp. 551-554, 2009.

[81] Y. Maeda, S. Kimura, Y. Hirashima, M. Kanda, Y. Lian, T. Wakahara, T. Akasaka, T. Hasegawa, H. Tokumoto, T. Shimizu, et al., "Dispersion of single-walled carbon nanotube bundles in nonaqueous solution," Journal of Physical Chemistry B, vol. 108, no. 48, pp. 18395-18397, 2004.

[82] S. Ravi, Properties of Single Walled Carbon Nanotube Films: A Thesis Submitted to the Victoria University of Wellington in Fulfilment of the Requirements for the Degree of Master of Science in Physics. Victoria University of Wellington, 2008.

[83] B. I. Rosario-Castro, E. J. Contés, M. Lebrón-Colón, M. A. Meador, G. Sánchez-Pomales, and C. R. Cabrera, "Combined electron microscopy and spectroscopy characterization of as-received, acid purified, and oxidized hipco single-wall carbon nanotubes," Materials Characterization, vol. 60, no. 12, pp. 1442-1453, 2009.

[84] Y. Miyata, Y. Maniwa, and H. Kataura, "Selective oxidation of semiconducting singlewall carbon nanotubes by hydrogen peroxide," The Journal of Physical Chemistry B, vol. 110, no. 1, pp. 25-29, 2006.

[85] M. A. Meitl, Y. Zhou, A. Gaur, S. Jeon, M. L. Usrey, M. S. Strano, J. A. Rogers, et al., "Solution casting and transfer printing single-walled carbon nanotube films," Nano Letters, vol. 4, no. 9, pp. 1643-1647, 2004.

[86] R. Tenent, T. Barnes, J. Bergeson, A. Ferguson, B. To, L. Gedvilas, M. Heben, and J. Blackburn, "Ultrasmooth, Large-Area, High-Uniformity, Conductive Transparent Single-Walled-Carbon-Nanotube Films for Photovoltaics Produced by Ultrasonic Spraying," Advanced Materials, vol. 21, no. 31, pp. 3210-3216, 2009.

[87] M. Majumder, C. Rendall, M. Li, N. Behabtu, J. Eukel, R. Hauge, H. Schmidt, and M. Pasquali, "Insights into the physics of spray coating of SWNT films," Chemical Engineering Science, 2009.

[88] T. Mustonen, J. Mäklin, K. Kordás, N. Halonen, G. Tóth, S. Saukko, J. Vähäkangas, H. Jantunen, S. Kar, P. Ajayan, R. Vajtai, P. Hellistö, H. Seppaä, and H. Moilanen, "Controlled ohmic and nonlinear electrical transport in inkjet-printed single-wall carbon nanotube films," Physical Review B, vol. 77, no. 12, p. 125430, 2008. 
[89] Z. Li, H. R. Kandel, E. Dervishi, V. Saini, Y. Xu, A. R. Biris, D. Lupu, G. J. Salamo, and A. S. Biris, "Comparative study on different carbon nanotube materials in terms of transparent conductive coatings," Langmuir, vol. 24, no. 6, pp. 2655-2662, 2008.

[90] M. S. Fuhrer, J. Nygård, L. Shih, M. Forero, Y. G. Yoon, H. J. Choi, J. Ihm, S. G. Louie, A. Zettl, and P. L. McEuen, "Crossed nanotube junctions," Science, vol. 288, no. 5465, pp. 494-497, 2000.

[91] C. Morgan, Z. Alemipour, and M. Baxendale, "Variable range hopping in oxygen-exposed single-wall carbon nanotube networks," Physica Status Solidi A, vol. 205, no. 6, pp. 13941398, 2008.

[92] V. Skákalová, A. B. Kaiser, Z. Osváth, G. Vértesy, L. P. Biró, and S. Roth, "Ion irradiation effects on conduction in single-wall carbon nanotube networks," Applied Physics A: Materials Science \& Processing, vol. 90, no. 4, pp. 597-602, 2008.

[93] J. S. Park, Y. Oyama, R. Saito, W. Izumida, J. Jiang, K. Sato, C. Fantini, A. Jorio, G. Dresselhaus, and M. S. Dresselhaus, "Raman resonance window of single-wall carbon nanotubes," Physical Review B, vol. 74, no. 16, p. 165414, 2006.

[94] J. Maultzsch, H. Telg, S. Reich, and C. Thomsen, "Radial breathing mode of single-walled carbon nanotubes: Optical transition energies and chiral-index assignment," Physical Review B, vol. 72, no. 20, p. 205438, 2005.

[95] P. T. Araujo, C. Fantini, M. M. Lucchese, M. S. Dresselhaus, and A. Jorio, "The effect of environment on the radial breathing mode of supergrowth single wall carbon nanotubes," Applied Physics Letters, vol. 95, no. 26, pp. 261902-261902, 2009.

[96] H. Kataura, Y. Kumazawa, Y. Maniwa, I. Umezu, S. Suzuki, Y. Ohtsuka, and Y. Achiba, "Optical properties of single-wall carbon nanotubes," Synthetic Metals, vol. 103, no. 1, pp. 2555-2558, 1999.

[97] C. Kramberger, H. Kuzmany, and W. Plank, "Temperature effects on the raman scattering of single-wall carbon nanotubes," in AIP Conference Proceedings, vol. 633 of STRUCTURAL AND ELECTRONIC PROPERTIES OF MOLECULAR NANOSTRUCTURES: XVI International Winterschool on Electronic Properties of Novel Materials, pp. 302-305, 2002.

[98] S. B. Cronin, Y. Yin, A. Walsh, R. B. Capaz, A. Stolyarov, P. Tangney, M. L. Cohen, S. G. Louie, A. K. Swan, M. S. Ünlü, B. B. Goldber, and M. Tinkham, "Temperature dependence of the optical transition energies of carbon nanotubes: The role of electron-phonon coupling and thermal expansion," Physical Review Letters, vol. 96, no. 12, pp. 127403$1-127493-4,2006$. 
[99] L. J. Li, R. J. Nicholas, R. S. Deacon, and P. A. Shields, "Chirality assignment of singlewalled carbon nanotubes with strain," Physical Review Letters, vol. 93, no. 15, p. 156104, 2004 .

[100] D. A. Heller, P. W. Barone, J. P. Swanson, R. M. Mayrhofer, and M. S. Strano, "Using raman spectroscopy to elucidate the aggregation state of single-walled carbon nanotubes," The Journal of Physical Chemistry B, vol. 108, no. 22, pp. 6905-6909, 2004.

[101] H. Mark and J. J. Workman, "Derivatives in spectroscopy," Spectroscopy, vol. 18, pp. 32$37,2003$.

[102] P. T. Araujo, P. B. C. Pesce, M. S. Dresselhaus, K. Sato, R. Saito, and A. Jorio, "Resonance raman spectroscopy of the radial breathing modes in carbon nanotubes," Physica E: Low-dimensional Systems and Nanostructures, vol. 42, no. 5, pp. 1251-1261, 2010.

[103] P. B. C. Pesce, P. T. Araujo, P. Nikolaev, S. K. Doorn, K. Hata, R. Saito, M. S. Dresselhaus, and A. Jorio, "Calibrating the single-wall carbon nanotube resonance raman intensity by high resolution transmission electron microscopy for a spectroscopy-based diameter distribution determination," Applied Physics Letters, vol. 96, p. 051910, 2010.

[104] M. Ashkin, J. H. Parker, and D. W. Feldman, "Temperature dependence of the raman lines of $\alpha \mathrm{al}_{2} \mathrm{O}_{3}, "$ Solid State Communications, vol. 6, pp. 343-346, 1968.

[105] S. P. S. Porto and R. S. Krishnan, "Raman effect of corundum," The Journal of Chemical Physics, vol. 47, p. 1009, 1967.

[106] P. Corio, A. Jorio, N. Demir, and M. S. Dresselhaus, "Spectro-electrochemical studies of single wall carbon nanotubes films," Chemical Physics Letters, vol. 392, no. 4, pp. 396402, 2004.

[107] K. P. Meletov, A. V. Krestinin, J. Arvanitidis, D. Christofilos, and G. A. Kourouklis, "Thermally induced softening of the radial breathing modes of bundled single-walled carbon nanotubes," Fullerenes, Nanotubes, and Carbon Nanostructures, vol. 18, no. 4-6, pp. 538-544, 2010.

[108] T. Uchida, M. Tazawa, H. Sakai, A. Yamazaki, and Y. Kobayashi, "Radial breathing modes of single-walled carbon nanotubes in resonance raman spectra at high temperature and their chiral index assignment," Applied Surface Science, vol. 254, no. 23, pp. 7591$7595,2008$.

[109] Y. Zhang, L. Xie, J. Zhang, Z. Wu, and Z. Liu, "Temperature coefficients of raman frequency of individual single-walled carbon nanotubes," The Journal of Physical Chemistry $C$, vol. 111, no. 38, pp. 14031-14034, 2007. 
[110] Z. Zhou, X. Dou, L. Ci, L. Song, D. Liu, Y. Gao, J. Wang, L. Liu, W. Zhou, and S. Xie, "Temperature dependence of the raman spectra of individual carbon nanotubes," The Journal of Physical Chemistry B, vol. 110, no. 3, pp. 1206-1209, 2006.

[111] V. A. Karachevtsev, A. Y. Glamazda, U. Dettlaff-Weglikowska, V. S. Kurnosov, E. D. Obraztsova, A. V. Peschanskii, V. V. Eremenko, and S. Roth, "Raman spectroscopy of hipco single-walled carbon nanotubes at 300 and 5 k," Carbon, vol. 41, no. 8, pp. 1567$1574,2003$.

[112] N. R. Raravikar, P. Keblinski, A. M. Rao, M. S. Dresselhaus, L. S. Schadler, and P. M. Ajayan, "Temperature dependence of radial breathing mode raman frequency of singlewalled carbon nanotubes," Physical Review B, vol. 66, no. 23, p. 235424, 2002.

[113] A. Jorio, C. Fantini, M. S. S. Dantas, M. A. Pimenta, A. G. Souza Filho, G. G. Samsonidze, V. W. Brar, G. Dresselhaus, M. S. Dresselhaus, A. K. Swan, M. S. Ünlü, B. B. Goldberg, and R. Saito, "Linewidth of the raman features of individual single-wall carbon nanotubes," Physical Review B, vol. 66, p. 115411, 2002.

[114] M. N. Iliev, A. P. Litvinchuk, S. Arepalli, P. Nikolaev, and C. D. Scott, "Fine structure of the low-frequency raman phonon bands of single-wall carbon nanotubes," Chemical Physics Letters, vol. 316, no. 3, pp. 217-221, 2000.

[115] Y. Maniwa, R. Fujiwara, H. Kira, H. Tou, H. Kataura, S. Suzuki, Y. Achiba, E. Nishibori, M. Takata, M. Sakata, A. Fujiwara, and H. Suematsu, "Thermal expansion of singlewalled carbon nanotube (swnt) bundles: X-ray diffraction studies," Physical review B, vol. 64, no. 24, p. 241402, 2001.

[116] L. Ci, Z. Zhou, L. Song, X. Yan, D. Liu, H. Yuan, Y. Gao, J. Wang, L. Liu, W. Zhou, G. Wang, and S. Xie, "Temperature dependence of resonant raman scattering in doublewall carbon nanotubes," Applied Physics Letters, vol. 82, no. 18, pp. 3098-3100, 2003.

[117] Q. Zhang, D. J. Yang, S. G. Wang, S. F. Yoon, and J. Ahn, "Influences of temperature on the raman spectra of single-walled carbon nanotubes," Smart materials and structures, vol. 15 , p. S1, 2006.

[118] I. Chatzakis, H. Yan, D. Song, S. Berciaud, and T. F. Heinz, "Temperature dependence of the anharmonic decay of optical phonons in carbon nanotubes and graphite," Physical Review B, vol. 83, no. 20, p. 205411, 2011.

[119] L. Song, W. Ma, Y. Ren, W. Zhou, S. Xie, P. Tan, and L. Sun, "Temperature dependence of raman spectra in single-walled carbon nanotube rings," Applied Physics Letters, vol. 92, p. $121905,2008$.

[120] F. Huang, K. T. Yue, P. Tan, S. L. Zhang, Z. Shi, X. Zhou, and Z. Gu, "Temperature dependence of the raman spectra of carbon nanotubes," Journal of Applied Physics, vol. 84, no. 7, pp. 4022-4024, 1998. 
[121] K. Gao, R. Dai, Z. Zhang, and Z. Ding, "Anharmonic effects in single-walled carbon nanotubes," Journal of Physics: Condensed Matter, vol. 19, p. 486210, 2007.

[122] J. Marquina, C. H. Power, J. M. Broto, E. Flahaut, and J. Gonzalez, "Anharmonic properties of raman modes in double wall carbon nanotubes," Revista Mexicana de Física, vol. 57, no. 6, pp. 510-517, 2011.

[123] Z. Zhou, L. Ci, L. Song, X. Yan, D. Liu, H. Yuan, Y. Gao, J. Wang, L. Liu, W. Zhou, S. Xie, Y. Du, and Y. Mo, "The intrinsic temperature effect of raman spectra of doublewalled carbon nanotubes," Chemical Physics Letters, vol. 396, no. 4, pp. 372-376, 2004.

[124] T. Lan, X. Tang, and B. Fultz, "Phonon anharmonicity of rutile tio_ $\{2\}$ studied by raman spectrometry and molecular dynamics simulations," Physical Review B, vol. 85, no. 9, p. 094305, 2012.

[125] N. Bonini, M. Lazzeri, N. Marzari, and F. Mauri, "Phonon anharmonicities in graphite and graphene," Physical Review Letters, vol. 99, no. 17, p. 176802, 2007.

[126] M. Yin, C. K. Wu, Y. Lou, C. Burda, J. T. Koberstein, Y. Zhu, and S. O'Brien, "Copper oxide nanocrystals," Journal of the American Chemical Society, vol. 127, no. 26, pp. 95069511, 2005.

[127] W. E. Garner, T. J. Gray, F. S. Stone, S. D. Savage, and P. F. Tiley, "Reactions on the surface of copper oxide," Discussions of the Faraday Society, vol. 8, no. 0, pp. 246-258, 1950 .

[128] X. G. Zheng, C. N. Xu, Y. Tomokiyo, E. Tanaka, H. Yamada, and Y. Soejima, "Observation of charge stripes in cupric oxide," Physical Review Letters, vol. 85, no. 24, pp. 5170-5173, 2000.

[129] S. Asbrink and L. J. Norrby, "A refinement of the crystal structure of copper (ii) oxide with a discussion of some exceptional esd's," Acta Crystallographica Section B: Structural Crystallography and Crystal Chemistry, vol. 26, no. 1, pp. 8-15, 1970.

[130] D. Wu, Q. Zhang, and M. Tao, "LSDA + U study of cupric oxide: Electronic structure and native point defects," Physical Review B, vol. 73, p. 235206, 2006.

[131] B. Meyer, A. Polity, D. Reppin, M. Becker, P. Hering, P. Klar, T. Sander, C. Reindl, J. Benz, M. Eickhoff, et al., "Binary copper oxide semiconductors: From materials towards devices," Physica Status Solidi (B), vol. 249, pp. 1487-1509, 2012.

[132] F. P. Koffyberg and F. A. Benko, "A photoelectrochemical determination of the position of the conduction and valence band edges of p-type cuo," Journal of Applied Physics, vol. 53, no. 2, pp. 1173-1177, 1982. 
[133] J. C. Irwin, J. Chrzanowski, T. Wei, D. J. Lockwood, and A. Wold, "Raman scattering from single crystals of cupric oxide," Physica C: Superconductivity, vol. 166, no. 5-6, pp. 456-464, 1990.

[134] V. Figueiredo, E. Elangovan, G. Gonçalves, N. Franco, E. Alves, S. Park, R. Martins, and E. Fortunato, "Electrical, structural and optical characterization of copper oxide thin films as a function of post annealing temperature," Physica Status Solidi (A), vol. 206, no. 9, pp. 2143-2148, 2009.

[135] S. B. Ogale, P. G. Bilurkar, N. Mate, S. M. Kanetkar, N. Parikh, and B. Patnaik, "Deposition of copper oxide thin films on different substrates by pulsed excimer laser ablation," Journal of Applied Physics, vol. 72, no. 8, pp. 3765-3769, 1992.

[136] K. Reimann and K. Syassen, "Pressure dependence of raman modes in cuo," Solid State Communications, vol. 76, pp. 137-140, October 1990.

[137] X. Jiang, T. Herricks, and Y. Xia, "CuO nanowires can be synthesized by heating copper substrates in air," Nano letters, vol. 2, no. 12, pp. 1333-1338, 2002.

[138] D. Chen, G. Shen, K. Tang, and Y. Qian, "Large-scale synthesis of cuo shuttle-like crystals via a convenient hydrothermal decomposition route," Journal of crystal growth, vol. 254, no. 1, pp. 225-228, 2003.

[139] J. C. Park, J. Kim, H. Kwon, and H. Song, "Gram-scale synthesis of cu2o nanocubes and subsequent oxidation to cuo hollow nanostructures for lithium-ion battery anode materials," Advanced Materials, vol. 21, no. 7, pp. 803-807, 2009.

[140] L. Yu, G. Zhang, Y. Wu, X. Bai, and D. Guo, "Cupric oxide nanoflowers synthesized with a simple solution route and their field emission," Journal of Crystal Growth, vol. 310, no. 12, pp. 3125-3130, 2008.

[141] S. Manna, K. Das, and S. K. De, "Template-free synthesis of mesoporous cuo dandelion structures for optoelectronic applications," ACS Applied Materials \& Interfaces, vol. 2, no. 5, pp. 1536-1542, 2010.

[142] Y. W. Zhu, C. H. Sow, and J. T. L. Thong, "Enhanced field emission from cuo nanowire arrays by in situ laser irradiation," Journal of Applied Physics, vol. 102, no. 11, p. 114302, 2007.

[143] D. Li, J. Hu, R. Wu, and J. Lu, "Conductometric chemical sensor based on individual cuo nanowires," Nanotechnology, vol. 21, p. 485502, 2010.

[144] J. Chrzanowski and J. C. Irwin, "Raman scattering from cupric oxide," Solid State Communications, vol. 70, no. 1, pp. 11-14, 1989.

[145] J. C. Irwin and T. Wei, "Raman scattering investigation of $\mathrm{cu}^{18} \mathrm{o}$, " Journal of Physics: Condensed Matter, vol. 3, p. 299, 1991. 
[146] H. F. Goldstein, D. Kim, P. Y. Yu, L. C. Bourne, J. P. Chaminade, and L. Nganga, "Raman study of cuo single crystals," Physical Review B, vol. 41, no. 10, pp. 7192-7194, 1990.

[147] Z. Wang, V. Pischedda, S. K. Saxena, and P. Lazor, "X-ray diffraction and raman spectroscopic study of nanocrystalline cuo under pressures," Solid state communications, vol. 121, no. 5, pp. 275-279, 2002.

[148] M. H. Chou, S. B. Liu, C. Y. Huang, S. Y. Wu, and C. L. Cheng, "Confocal raman spectroscopic mapping studies on a single cuo nanowire," Applied Surface Science, vol. 254, no. 23, pp. 7539-7543, 2008.

[149] J. F. Xu, W. Ji, Z. X. Shen, W. S. Li, S. H. Tang, X. R. Ye, D. Z. Jia, and X. Q. Xin, "Raman spectra of cuo nanocrystals," Journal of Raman Spectroscopy, vol. 30, no. 5, pp. 413-415, 1999.

[150] Y. L. Huang, M. H. Chou, S. Y. Wu, and C. L. Cheng, "Investigation of quantumconfinement effect in a single cuo nanowire," Japanese Journal of Applied Physics, vol. 47, no. 1, pp. 703-705, 2008.

[151] H. Fan, B. Zou, Y. Liu, and S. Xie, "Size effect on the electron-phonon coupling in cuo nanocrystals," Nanotechnology, vol. 17, p. 1099, 2006.

[152] H. Richter, Z. Wang, and L. Ley, "The one phonon raman spectrum in microcrystalline silicon," Solid State Communications, vol. 39, no. 5, pp. 625-629, 1981.

[153] K. W. Adu, H. R. Gutierrez, U. J. Kim, and P. C. Eklund, "Inhomogeneous laser heating and phonon confinement in silicon nanowires: A micro-raman scattering study," Physical Review B, vol. 73, no. 15, p. 155333, 2006.

[154] G. Faraci, S. Gibilisco, and A. R. Pennisi, "Quantum confinement and thermal effects on the raman spectra of si nanocrystals," Physical Review B, vol. 80, no. 19, p. 193410, 2009 .

[155] Z. Wang, J. E. Alaniz, W. Jang, J. E. Garay, and C. Dames, "Thermal conductivity of nanocrystalline silicon: Importance of grain size and frequency-dependent mean free paths," Nano letters, vol. 11, p. 22062213, 2011.

[156] H. Yamada, X. G. Zheng, Y. Soejima, and M. Kawaminami, "Lattice distortion and magnetolattice coupling in cuo," Physical Review B, vol. 69, no. 10, p. 104104, 2004.

[157] N. A. Mohemmed Shanid, M. Abdul Khadar, and V. G. Sathe, "Frohlich interaction and associated resonance enhancement in nanostructured copper oxide films," Journal of Raman Spectroscopy, vol. 42, no. 9, pp. 1769-1773, 2011.

[158] X. K. Chen, J. C. Irwin, and J. P. Franck, "Evidence for a strong spin-phonon interaction in cupric oxide," Physical Review B, vol. 52, no. 18, pp. 13130-13133, 1995. 
[159] W. Y. Ching, Y.-N. Xu, and K. W. Wong, "Ground-state and optical properties of $\mathrm{cu}_{2} \mathrm{O}$ and cuo crystals," Physical Review B, vol. 40, pp. 7684-7695, Oct 1989.

[160] D. M. Riffe, "Classical fano oscillator," Physical Review B, vol. 84, p. 064308, Aug 2011.

[161] B. G. Burke, J. Chan, K. A. Williams, Z. Wu, A. A. Puretzky, and D. B. Geohegan, "Raman study of fano interference in p-type doped silicon," Journal of Raman Spectroscopy, vol. 41, no. 12, pp. 1759-1764, 2010.

[162] R. Gupta, Q. Xiong, C. K. Adu, U. J. Kim, and P. C. Eklund, "Laser-induced fano resonance scattering in silicon nanowires," Nano Letters, vol. 3, no. 5, pp. 627-631, 2003.

[163] W. Reichardt, F. Gompf, M. Ain, and B. M. Wanklyn, "Lattice dynamics of cupric oxide," Zeitschrift für Physik B Condensed Matter, vol. 81, no. 1, pp. 19-24, 1990.

[164] D. Li, Y. Wu, P. Kim, L. Shi, P. Yang, and A. Majumdar, "Thermal conductivity of individual silicon nanowires," Applied Physics Letters, vol. 83, p. 2934, 2003.

[165] H. Scheel, S. Reich, A. C. Ferrari, M. Cantoro, A. Colli, and C. Thomsen, "Raman scattering on silicon nanowires: The thermal conductivity of the environment determines the optical phonon frequency," Applied Physics Letters, vol. 88, no. 23, pp. 233114233114, 2006.

[166] R. Jalilian, G. U. Sumanasekera, H. Chandrasekharan, and M. Sunkara, "Phonon confinement and laser heating effects in germanium nanowires," Physical Review B, vol. 74, no. 15 , p. $155421,2006$.

[167] D. http://www.rafoeg.de/20, Dokumentenarchiv/20

[168] C. H. Xu, X. L. Yang, S. Q. Shi, Y. Liu, C. Surya, and C. H. Woo, "Effects of local gas-flow field on synthesis of oxide nanowires during thermal oxidation," Applied Physics Letters, vol. 92, no. 25, p. 253117, 2008.

[169] A. M. B. Gonçalves, L. C. Campos, A. S. Ferlauto, and R. G. Lacerda, "On the growth and electrical characterization of $\mathrm{CuO}$ nanowires by thermal oxidation," Journal of Applied Physics, vol. 106, p. 034303, 2009.

[170] A. Kumar, A. K. Srivastava, P. Tiwari, and R. V. Nandedkar, "The effect of growth parameters on the aspect ratio and number density of cuo nanorods," Journal of Physics: Condensed Matter, vol. 16, p. 8531, 2004.

[171] C. H. Xu, C. H. Woo, and S. Q. Shi, "Formation of cuo nanowires on cu foil," Chemical physics letters, vol. 399, no. 1, pp. 62-66, 2004.

[172] C. H. Xu, C. H. Woo, and S. Q. Shi, "The effects of oxidative environments on the synthesis of cuo nanowires on cu substrates," Superlattices and Microstructures, vol. 36, no. 1, pp. 31-38, 2004. 
[173] B. H. O. O'Connor, D. Y. Li, and B. A. Hunter, "The importance of the specimen displacement correction in rietveld pattern fitting with symmetric reflection-optics diffraction data," Advances in X-Ray Analysis, vol. 44, pp. 96-102, 2001.

[174] A. Bianchi, L. Montenegro, R. Viña, and G. Punte, "Microstructure anisotropy in cuo powders," Powder Diffraction, vol. 23, p. S81, 2008.

[175] F. Bayansal, S. Kahraman, G. Çankaya, H. Çetinkara, H. Guder, and H. Çakmak, "Growth of homogenous $\mathrm{CuO}$ nano-structured thin films by a simple solution method," Journal of Alloys and Compounds, vol. 509, no. 5, pp. 2094-2098, 2010.

[176] S. Sarkar, P. Jana, and B. Chaudhuri, "Colossal internal barrier layer capacitance effect in polycrystalline copper (II) oxide," Applied Physics Letters, vol. 92, p. 022905, 2008.

[177] T. Serin, A. Yildiz, S. Horzum Sahin, and N. Serin, "Extraction of important electrical parameters of CuO," Physica B: Condensed Matter, vol. 406, no. 3, pp. 575-578, 2010.

[178] http://rsbweb.nih.gov/ij/

[179] http://www.mathworks.com/matlabcentral/fileexchange/9168

[180] M. S. Yeh, Y. S. Yang, Y. P. Lee, H. F. Lee, Y. H. Yeh, and C. S. Yeh, "Formation and characteristics of cu colloids from cuo powder by laser irradiation in 2-propanol," The Journal of Physical Chemistry B, vol. 103, no. 33, pp. 6851-6857, 1999.

[181] G. K. Wertheim, M. A. Butler, K. W. West, and D. N. E. Buchanan, "Determination of the gaussian and lorentzian content of experimental line shapes," Review of Scientific Instruments, vol. 45, no. 11, pp. 1369-1371, 1974.

[182] M. Ohashi, A. Tashiro, G. Oomi, E. Maeda, and X.-G. Zheng, "Effect of pressure on the magnetic phase transition in cupric oxide," Physical Review B, vol. 73, p. 134421, Apr 2006 .

[183] R. Tubino, L. Piseri, and G. Zerbi, "Lattice dynamics and spectroscopic properties by a valence force potential of diamondlike crystals: C, si, ge, and sn," The Journal of Chemical Physics, vol. 56, p. 1022, 1972.

[184] W. Weber, "Adiabatic bond charge model for the phonons in diamond, si, ge, and $\alpha$-sn," Physical Review B, vol. 15, no. 10, p. 4789, 1977.

[185] T. Beechem, S. Graham, S. P. Kearney, L. M. Phinney, and J. R. Serrano, "Invited article: Simultaneous mapping of temperature and stress in microdevices using microraman spectroscopy," Review of Scientific Instruments, vol. 78, p. 061301, 2007.

[186] S. Kouteva-Arguirova, T. Arguirov, D. Wolfframm, and J. Reif, "Influence of local heating on micro-raman spectroscopy of silicon," Journal of Applied Physics, vol. 94, p. 4946, 2003. 
[187] S. Ravi, A. B. Kaiser, and C. W. Bumby, "Thin films of cupric oxide: Crystallite size and conduction mechanism analysis," in Materials Science Forum, vol. 700, pp. 71-79, Trans Tech Publications, 2012.

[188] R. J. Nemanich, D. K. Biegelsen, R. A. Street, and L. E. Fennell, "Raman scattering from solid silicon at the melting temperature," Physical Review B, vol. 29, pp. 6005-6007, May 1984.

[189] J. W. Vandersande and C. Wood, "The thermal conductivity of insulators and semiconductors," Contemporary Physics, vol. 27, no. 2, pp. 117-144, 1986.

[190] J. Reydellet, M. Balkanski, and D. Trivich, "Light scattering and infrared absorption in cuprous oxide," physica status solidi (b), vol. 52, no. 1, pp. 175-185, 1972.

[191] J. Raptis, E. Liarokapis, and E. Anastassakis, "Effect of temperature gradients on the first-order raman spectrum of si," Applied Physics Letters, vol. 44, no. 1, pp. 125-127, 1984.

[192] T. Yu, C. H. Sow, A. Gantimahapatruni, F. C. Cheong, Y. Zhu, K. C. Chin, X. Xu, C. T. Lim, Z. Shen, J. T. L. Thong, and A. T. S. Wee, "Patterning and fusion of cuo nanorods with a focused laser beam," Nanotechnology, vol. 16, no. 8, p. 1238, 2005.

[193] "http://www.reade.com/," 